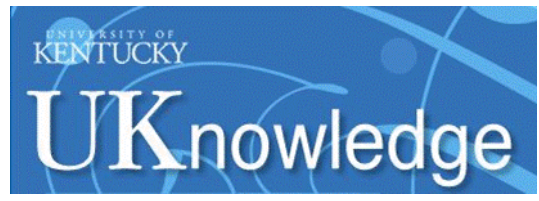

University of Kentucky

UKnowledge

\title{
PRACTICE AND APPLICATION OF KNOWLEDGE BY NUTRITION STUDENTS
}

Jennifer Kristin Boyee

University of Kentucky, jennifer.boyee@uky.edu

Right click to open a feedback form in a new tab to let us know how this document benefits you.

\section{Recommended Citation}

Boyee, Jennifer Kristin, "PRACTICE AND APPLICATION OF KNOWLEDGE BY NUTRITION STUDENTS"

(2011). University of Kentucky Master's Theses. 122.

https://uknowledge.uky.edu/gradschool_theses/122

This Thesis is brought to you for free and open access by the Graduate School at UKnowledge. It has been accepted for inclusion in University of Kentucky Master's Theses by an authorized administrator of UKnowledge. For more information, please contact UKnowledge@lsv.uky.edu. 


\section{ABSTRACT OF THESIS}

\section{PRACTICE AND APPLICATION OF KNOWLEDGE BY NUTRITION STUDENTS}

Practice and knowledge of upper-level human nutrition and dietetics students (juniors and seniors, $n=96$ ) attending a midwestern university were compared to their comprehension and application of the Scope of Dietetics Practice. The sample consisted of 96 students, primarily (72\%) in the 21 - 23 age category; females made up $83 \%$ and males comprised the remaining $17 \%$ of the sample. In this sample, $70 \%$ of the participants reported dietetics as their current major, and 30\% stated that their major was human nutrition. Overall, students majoring in human nutrition responded neutrally to the questions concerning comprehension, knowledge, and implementation of the Scope of Dietetics Practice, while participants majoring in dietetics reported better knowledge, comprehension, and implementation of the Scope of Dietetics Practice. However, students majoring in human nutrition reported increased use of nutrition information to diagnose others than were students studying dietetics. In general, participants responded to questions regarding their practice and application of nutrition knowledge to themselves and others as would be expected, though there were differences between the students studying human nutrition and those studying dietetics.

KEY WORDS: college students, Scope of Dietetics Practice, dietetics, Code of Ethics, nutrition advice

Jennifer Kristin Boyee May 3, 2011 
PRACTICE AND APPLICATION OF KNOWLEDGE BY NUTRITION STUDENTS

\author{
By \\ Jennifer Kristin Boyee
}

Hazel Forsythe, PhD, RD, LD Director of Thesis

Hazel Forsythe, PhD, RD, LD

Director of Graduate Studies

May 3, 2011

Date 


\section{RULES FOR THE USE OF THESIS}

Unpublished theses submitted for the Master's degree and deposited in the University of Kentucky Library are as a rule open for inspection, but are to be used only with due regard to the rights of the authors. Bibliographical references may be noted, but quotations or summaries of parts may be published only with the permission of the author, and with the usual scholarly acknowledgments.

Extensive copying or publication of the thesis in whole or in part also requires the consent of the Dean of the Graduate School of the University of Kentucky.

A library that borrows this thesis for use by its patrons is expected to secure the signature of each user.

Name

Date 


\section{THESIS}

Jennifer Kristin Boyee

The Graduate School

University of Kentucky

2011 
PRACTICE AND APPLICATION OF KNOWELDGE BY NUTRITION STUDENTS

\section{THESIS}

A thesis submitted in partial fulfillment of the requirements for the degree of Master of Sciences College of Agriculture at the University of Kentucky

$$
\begin{gathered}
\text { By } \\
\text { Jennifer Kristin Boyee } \\
\text { Lexington, KY }
\end{gathered}
$$

Director: Dr. Hazel Forsythe, RD, LD, Professor Lexington, Kentucky 2011

Copyright (C) Jennifer Kristin Boyee 2011 


\section{TABLE OF CONTENTS}

Table of Contents _ iii

List of Tables _iv

List of Figures $\quad v$

List of Files $\quad v i$

Chapter 1: Introduction $\quad 1$

Chapter 2: Literature Review

Weight Change in College Students 3

Collegiate Life, Stress, and Weight Gain 6

Nutrition Labeling and Personal Accountability $\quad 8$

Scope of Dietetics Practice $\quad 11$

Summary 13

Chapter 3: Methodology $\quad 14$

Research Purpose $\quad 14$

Research Questions $\quad 14$

Methodology $\quad 15$

Population and Sample Selection $\quad 15$

Research Design 17

Data Collection $\quad 18$

Measurements 18

Data Analysis 18

Chapter 4: Results $\quad 20$

Demographics $\quad 20$

Significant Correlations Regarding Scope of Dietetics Practice _ 23

Significant Correlations Involving Course of Study _ 36

Chapter 5: Discussion $\quad 46$

Scope of Dietetics Practice Implications $\quad 46$

Discussion $\quad 47$

Course of Study Implications $\quad 53$

Bias, Limitations, and Future Research $\quad 54$

Final Conclusions $\quad 55$

Appendices $\quad 57$

Appendix A: Survey $\quad 57$

Appendix B: Scope of Dietetics Practice Framework $\quad 72$

Appendix C: IRB Approval Documentation $\quad 73$

Appendix D: Statistical Analysis of Survey Results

References $\quad 246$

Vita 251 


\section{LIST OF TABLES}

Table 1: Demographics _ 22

Table 2: Scope of Practice 38

Table 3 (Part A): Solicited Advice 39

Table 3 (Part B): Solicited Advice 40

Table 4 (Part A): Unsolicited Advice

Table 4 (Part B): Unsolicited Advice 


\section{LIST OF FIGURES}

Figure 1: Scope of Dietetics Practice \#1 25

Figure 2: Scope of Dietetics Practice \#2 28

Figure 3: Scope of Dietetics Practice \#3 30

Figure 4: Scope of Dietetics Practice \#4 33

Figure 5: Scope of Dietetics Practice \#5 36

Figure 6: Solicited Advice:

Eat a high protein, low carbohydrate diet

Figure 7: Solicited Advice:

Make smart food choices when eating away from home

Figure 8: Unsolicited Advice:

Eat a high protein, low carbohydrate diet

Figure 9: Unsolicited Advice:

Make smart food choices when eating away from home 44

Figure 10: Stressor: Relationship Ends 


\section{LIST OF FILES}

1. JBoyeeThesis.pdf 6MB 


\section{Chapter 1: Introduction}

College years serve as a period of life changing decisions for many people across the United States. During this time, students make the majority of their choices without a great deal of outside influence - what to study, where to live, and what to eat, to name a few. Many students also struggle with their weight at some point during their years in college, even those studying nutrition. The American Dietetic Association states that registered dietitians (RD) are the nation's food and nutrition experts, but how does this expertise mix with the demands of college life in students studying to become registered dietitians?

The food service systems at universities and colleges are notorious for their calorically dense, nutritionally questionable food selections, as well as their encouragement of poor eating habits due to circumstances. As these foods contain high levels of calories and grams of fat, many university students experience weight gain during their freshman year of school. Studies have examined the validity of the "freshman fifteen" and one such study found that typical weight gain during the freshman year specifically averaged closer to about four pounds (Vella-Zarb, RA., Elgar, FJ., 2009). While some people gain fifteen or more pounds during their freshman year, it does not trend as the norm. Though five pounds of weight gain may not concern the general public, an addition of five pounds per year over the course of four years at the university would result in a net gain of twenty pounds, which is cause for concern. In order to prepare nutrition students to become experts in their field, professors must relay to them the importance of maintaining a healthy 
weight-for-height throughout all stages of their lives and work with their students to make healthy choices in difficulty scenarios, like a college dining facility.

In addition to managing their own weight management, friends and family often ask for nutrition and health advice from nutrition students by the time the student reaches the upper level nutrition courses. At this level of academic preparation, family members, friends, and acquaintances often assume that the student studying nutrition has knowledge comparable to a dietitian. While students may have been studying nutrition topics for many years and are able to answer general inquiries, it is important for the general public to understand that students do not have the same knowledge base as a dietitian. To answer questions appropriately, students must know and identify with the American Dietetic Association's Scope of Practice, as well as their course content.

Students must possess the knowledge to live as a nutrition expert. The intent of this study is to examine how nutrition students utilize their knowledge in their daily lives by investigating their food-related behavior and nutrition recommendations. By studying the practice and application of food-related behaviors and attitudes of upper level nutrition students, educational methods and content will be identified to better prepare future experts in the field of nutrition, the registered dietitians. 


\section{Chapter 2: Literature Review}

Weight Change in College Students

Students face many life decisions during their years in college. They have the opportunity to re-examine their beliefs and attitudes, make important decisions about the direction they want their lives to take, and hold complete responsibility for their personal health and well-being. These individual decisions were based on learned behaviors from youth; researchers Branen and Fletcher (1999) compared college students' current eating habits to their childhood recollections. They found that college students habits, including eating all of the food on their plate, consuming dessert, having meals at a regular schedule and using food as an incentive depended on the practices used by their childhood primary caregiver; additionally, current consideration of nutrition in selecting food items depended on their childhood primary caregiver's emphasis of nutrition and health (Pullman, AW, et al, 2009). This study proves the existence of a relationship between childhood eating habits and adult eating habits, reinforcing the idea that parents greatly influence their children's food-related behaviors, even after the children become adults and move away from home.

When students disregard the importance of good nutrition and exercise habits, the result typically manifests itself as weight gain. Nutrition and exercise tend to be forgotten because students find that eating healthfully and finding time for exercise can be a challenge. Exercise is easily forgotten because students do not understand its importance and believe that putting their time and energy into their studies and extra-curricular activities will benefit them in the long run more so than 
working out on a regular basis. Strong, et al (2008) identified that young adults comprising college populations are more at risk for unwanted weight gain than young adults not attending college. In their study, Strong, et al completed forty-three quantitative assessments comprised of body weight and composition, cardio respiratory fitness, and diet and activity habits, as well as structured qualitative assessments, consisting of structured interviews and focus groups. Through these assessments, the researchers found that college students do not highly prioritize eating healthfully or doing regular physical activity "despite having ample free time, high exercise self-efficacy, positive outcome expectations for exercise, and a desire to exercise more" (Strong, et al, 2008, p. 1708). The researchers concluded that "this population may not have adequate self-regulatory skills, such as planning and self-monitoring, to maintain healthful behaviors in the college environment." According to this study, while young adults in college know what they should do to maintain a healthy weight and lifestyle, they clearly do not prioritize health and wellness as highly as they should.

Transitioning from home life to college life is a dramatic change, a time often associated with weight gain. Many people are quick to blame college dining halls, particularly those set up as all-you-can-eat. A study by Levitsky, Halbmaier, and Mrdjenovic (2004) discovered otherwise. According to their research article on freshman weight gain eating breakfast and lunch at a campus 'all-you-can-eat' dining facility accounted for $20 \%$ of the total weight variance in their first model; however, this particular variable lost statistical significance once initial body weight was combined as a covariate. In their research, Levitsky, Halbmaier, and Mrdjenovic 
(2004) found that when initial body weight was implemented as a covariate, the statistically significant predictor variables were "number of evening snacks, number of meals consumed on the weekend, consumption of junk foods, and recent dieting." This indicates that students can eat at "all-you-can-eat" locations without gaining a disproportionate amount of weight. Nelson, et al, (2009) mimicked the results of this study; they discovered the following:

Major themes that emerged in describing important influences on weight, dietary intake, and physical activity included: unhealthful food availability on campus, snacking, late-night eating, alcohol-related eating, eating because of stress/boredom, and food in student dorm rooms. Other factors related to physical activity included: negative experiences using campus recreation facilities; poor weather; and lack of time/time management, motivation, and social support for exercise. (Nelson, et al, 2009, p. 287)

Though meal consumption at "all-you-can-eat" dining halls failed to carry statistical research significance in the study by Levitsky, Halbmaier, and Mrdjenovic (2004), the researchers noted that questionnaires completed by the students who ate at "all-you-can-eat" facilities indicated increased feeling of "fullness;" this suggests that these portions were considerably larger than traditional eating establishments. In the study by Levitsky and Youn (2004), the findings supported the hypothesis that increased portion size leads to overeating; the more food served to the participants, the more they consumed. While eliminating "all-you-can-eat" dining facilities is impractical because of their popularity with students, universities can add nutrition information through labeling, as well as teach students through their campus residence programming about the connection between nutrition and health. These would both be measures that could improve the health of students. 
The "freshman fifteen," a frequently used term to describe the typical perceived weight gain in college freshmen, serves as the primary example of how college students allow their health to take a backseat to all other demands of collegiate life. Not all college freshmen gain fifteen pounds during their first year away from home; however, the literature describes concepts that examine these changes and what enables weight gain. In a 2008 study regarding exercise habits among college students, researchers examined ways to prevent weight gain in the college population. Mihalopoulos (2008) discovered that participants had a difficult time incorporating exercise into their every-day schedule. Research explained this as "poor planning/time management, satisfaction with body image, lack of accountability, and feelings of laziness" (Strong et al, 2008, p. 1708). Furthermore, the findings "suggest that this population may not have adequate self-regulatory skills, such as planning and self-monitoring, to maintain healthful behaviors in the college environment" (Strong et al, 2008, p. 1708). The researchers found that, while students knew that exercise was good for them and important to include in their lives, exercise did not rate highly on their list of priorities.

\section{Collegiate Life, Stress, and Weight Gain}

As discussed previously, many college students experience weight gain because of their decreased prioritization of exercise, increased availability of high calorie, high fat foods, and the a decreased emphasis on consuming appropriate portion sizes in university dining halls. In addition to these factors, college serves as a stressful time; prior experimentation (Liu, et al, 2007; Wardle, Steptoe, Oliver, and Lipsey, 1999; and Weingarten and Elston, 1991) has been done at length regarding 
college students and stress, stress and food-related behaviors, and college students and food-related behaviors. A 2007 study investigated stress levels in college students; the researchers found that the level of stress a college freshman experiences in their adjustment is comparable to that of a student in their first year of medical or law school (Pritchard, Wilson, and Yamnitz, 2007). In their surveys of 242 undergraduate freshmen at the beginning and end of their first year in college, Pritchard, Wilson, and Yamnitz discovered that "data replicated the declines reported in law and medical students' psychological and physical health. Negative coping tactics and perfectionism predicted poorer physical health and alcohol use at the end of the year; however, optimism and self-esteem predicted better physical and psychological outcomes" (p. 15). Implications for this study primarily indicate the need for freshmen-geared programming regarding stress management and making health-conscious life choices.

Regarding stress and food-related behaviors, researchers, such as Kiecolt-Glaser (2010); Liu, et al (2007); and Wardle, Steptoe, Oliver, and Lipsey (1999); among others, found through several studies that stress increases poor nutrition choices, stating that "depression and stressful events motivate less healthy food choices" (Kiecolt-Glaser, 2010, p. 3). Furthermore, Torres and Nowson (2007) found that stressful life conditions seem to be connected to a desire for foods high in energy especially sugar and fat which means they may cause weight gain. The research also predicted that eating because of stress may be linked to obesity because of the increased desire to consume foods dense in fat, calories, and sugar. 


\section{Nutrition Labeling and Personal Accountability}

Good nutrition habits and knowledge are equally important in maintaining a healthy weight, along with consuming appropriate amounts and types of food and managing stress, as discussed above. The best way to build better nutrition habits and knowledge is through reading food labels. The National Labeling and Education Act served as a major milestone in the nutrition field by establishing standard terminology and requiring consistent content and lay out of nutrition facts labels ("Nutrition Labeling," 2010); however, people have to read and comprehend the labels for them to be of any use. Because people of all ages, genders, and ethnicities have difficulty navigating and achieving good nutritional health, it is now more important than ever for everyone studying nutrition to know how to model good behaviors and what advice is permissible with varying levels of education.

One area in which many people seek information is nutrition labeling. Although the notion of nutrition labeling was put into practice to help consumers make wise choices in selecting healthy foods, the concept is sometimes difficult to understand. In November of 1990, then-President George H.W. Bush signed into law the Nutrition Labeling and Education Act. This was an important reform act with the following provisions:

- Requires food manufacturers to disclose the fat (saturated and unsaturated), cholesterol, sodium, sugar, fiber, protein and carbohydrate content in their products;

- Requires labeling for the top-20-selling fruits, vegetables, fish and shellfish. Retailers may provide this information in a single location in their stores;

- Exempts from labeling such foods as meat, poultry and egg products, food sold in restaurants and at prepared food counters in grocery 
stores, infant formula, foods sold in bulk, foods with insignificant amounts of nutrients and foods sold by retailers with total sales of less than $\$ 500,000$;

- Requires the Food and Drug Administration to establish standards and definitions for food descriptors such as "low," "lean," "lite," "reduced," etc;

- Sets standards for allowing health claims on foods if the claims are based on sound scientific evidence and are truthful, accurate, and not misleading and for allowing third party references or endorsements. ("Nutrition Labeling," 2010, l. 18-35)

On January 6, 1993, the Food and Drug Administration (FDA) and the United States Department of Agriculture (USDA) released identical regulations regarding the format and content of nutrition labels on most foods. In May 2000, the USDA and the US Department of Health and Human Services updated the guidelines for Americans with the newer format that emphasizes physical activity, along with healthy eating, as an important component of a healthy lifestyle. Most recently, in January 2006, the Nutrition Facts Labels on all packaged food products must contain the amount of trans fatty acid, or trans fat, within one serving of the product. (“Nutrition Labeling," 2010)

The United States government regulations made strides in unifying the layout, content, and requirements of food labels; however, disadvantages persist. The main disadvantage of food labels is that people continue to avoid using them, likely because they do not understand the information and its application to their health. Nayga (2000) found that nutrition knowledge does not have an effect on food label use; when nutrition knowledge is adjusted for, men and women use food labels equally, though without this adjustment, men reported using labels less than 
women. Nayga (2000) summarized the use of nutrition labels by college students as "students want nutrition labels and would use them to make food purchasing decisions" (p. 109.) This statement applies to the United States population in that people who want nutrition labels will search out, find, and implement them in making food choices.

Personal accountability remains vital to maintaining good health, and asking for advice from friends and family members, especially those in the nutrition field, is one way to accrue knowledge and gain insight in achieving and maintaining good health. However, how much of their own knowledge and insight do nutrition students utilize in personal health practice and communication about nutrition to family and individuals? When asked for advice, students must avoid behaviors such as diagnosing health conditions. Nutrition educators make the assumption that nutrition students hold accurate information, but do students implement this knowledge in their personal health maintenance? Research shows that knowledge does not always translate to practice in the regular population (Nayga, 2000 and van Dillen et al, 2007).

In a study at a midwestern university, researchers Driskell, Schake, and Detter (2008) investigated the use of nutrition labeling in a university-dining hall to change the eating habits of its patrons. Through a cross-sectional study of 205 customers at a campus-dining hall, the researchers discovered that a significantly higher percent of women used the labeling than men. The primary reasons that patrons gave for not employing the labeling were that it would not change their food or meal selection and that they did not have enough time to consider it. The main 
reasons for using the labels included "general knowledge, concern about overall health, calorie counting, and concern about a certain nutrient(s)" (Driskell, Schake, and Detter, 2008, p. 2071). The study concluded that the majority of dining hall patrons were aware of the information and its availability; however, the researchers suggested that, because the information did not change many patrons' selections, that the connection between nutrition, label-reading, and good health are not widely understood in this population. (Driskell, Schake, and Detter, 2008)

\section{Scope of Dietetics Practice}

In 2005, the American Dietetic Association published the Scope of Dietetics Practice Framework (see Appendix B); the framework "serves as a cornerstone for the profession. The framework is a flexible decision-making structure that empowers practitioners to provide safe, effective, and timely health care services" (Maillet, Skates, Pritchett, 2005, p. 634). In other words, the framework exists to help dietetics professionals make ethical decisions and provide appropriate services to patients and clients.

There are three main parts to the framework: foundation knowledge, evaluation resources, and decision aids. The foundation knowledge defines the profession of dietetics, five characteristics of the profession, and establishes educational resources. As defined in the position paper by the America Dietetics Association, dietetics is "is the integration and application of principles derived from the sciences of food, nutrition, management, communication, and biological, physiological, behavioral, and social services to achieve and maintain optimal 
human health" (p. 635). In addition to defining the profession, this first block of foundation knowledge includes the five key characteristics of dietetics professionals and provides examples regarding the personification of the five characteristics: code of ethics, body of knowledge, education, autonomy, and service.

Once the foundation of the profession has been explained through the first block of the framework, the second block continues and outlines the code of ethics and standards of practice and professional performance for dietitians and registered dietetic technicians (DTRs), as well as specialty or advanced professionals (Maillet, Skates, Pritchett, 2005). Through this second block, practitioners and their managers can evaluate and gauge dietitian and dietetic technician performance. Furthermore, the Code of Ethics "reflect[s] the values and ethical principles guiding the dietetics profession and set[s] forth commitments and obligations of the dietetics practitioner to the public, clients, the profession, colleagues, and other professionals" (American Dietetic Association/Commission on Dietetic Registration, 2009, p. 1461). Through the Code of Ethics, individuals at varying levels of dietetics education and credentialing know to what responsibilities they are legally and ethically bound.

Finally, the third block serves as a decision making aid through a decision tree, decision analysis tools, definition of terms, and other resources that apply to dietetic-related situations. These decision tools help the dietetics professional to make safe, effective, and ethical choices in a wide range of situations. As discussed in the ADA's position paper, these tools are quite useful when state, federal, and ADA documents do not clearly delineate responsibility (Maillet, Skates, Pritchett, 2005). 
Summary

Does the assumption of nutrition, label-reading, and good health being understood by a limited part of the population applied to non-nutrition students apply to students studying nutrition? In other words, do students majoring in nutrition practice what they learn in courses and what they preach to others? According to the current research, nothing currently exists that examines the food-related behaviors of undergraduate students studying nutrition. However, college students overall tend to experience weight gain, undergo a great deal of stress, and have poor food-related behaviors. The purpose of the study is to examine the behaviors of undergraduate students studying nutrition and compare their beliefs and actions to their knowledge and practice of the Scope of Dietetics Practice. 


\section{Chapter 3: Methodology}

Research Purpose

The purpose of this research was to investigate whether nutrition students use their knowledge to deal with stressful situations, work within the Scope of Dietetics Practice, and model healthy food-related behaviors. If nutrition students do not model positive behaviors related to nutrition education delivery, the expectation is that their peers cannot seek, accept, and utilize their service as a source of advice. By examining undergraduate students' self-reported responses to stress, their food-related recommendations to others, their knowledge of the Dietetic Scope of Practice, and their ability to model positive behaviors to others, the dietetics community can reassess the current curriculum and determine if there are any changes necessary for the advancement of the profession.

\section{Research Questions}

\#1 Do undergraduate nutrition students utilize sound nutrition practices?

\#2 Does the health advice undergraduate nutrition students give to their friends and family fit the Scope of Dietetics Practice?

\#3 Do undergraduate nutrition students utilize food-related behaviors to cope with stress?

\#4 Are there any significant differences between students studying dietetics and those studying human nutrition in behaviors involving advice given to others, stress management within their own lives, and their food-related behaviors?

This study adds to the body of knowledge by exploring the behaviors of students studying nutrition in a large, southern university. This study seeks to understand 
nutrition students' advice to other people, their basic nutritional knowledge, and their use of sound nutrition practices in their own lives.

Methodology

Prior to commencement of this study, the survey (see Appendix A) was developed and approved through the University of Kentucky Office of Research Integrity. An application with the appropriate documents and forms was submitted for Institutional Review Board (IRB) approval in October 2010; the project earned approval in November 2010 and the survey opened after getting IRB permission.

This study used a quantitative design to assess the application and practice of knowledge by nutrition student; it utilized data collected during one survey available to respondents November 2010 through March 2011. The survey provided data regarding demographic information, advice the student gives to others, the student's response to stressful situations, and their knowledge of the Scope of Dietetics Practice. Each survey was made available to all Kentucky students enrolled in at least one upper-level course in nutrition or a Didactic Program in Dietetics course. All surveys were completed and returned for analysis.

\section{Population and Sample Selection}

The study population consisted of students enrolled in upper level nutrition and dietetics course throughout the State of Kentucky. In selecting universities in Kentucky to approach, the only schools chosen were those with a Didactic Program in Dietetics; the directors of each program were approached via e-mail with a 
request for student participation. After the initial e-mail, two follow-up notices were sent with requests for participation - one midway through the data collection period and one during the final week of data collection. Each e-mail message included the link for the survey, as well as the target population. In addition to completing the IRB approval process through the University of Kentucky, Western Kentucky required approval through their university research office.

The population consisted of students majoring in either Human Nutrition (or any pre-professional major involving nutrition) or Dietetics (or the major leading students to complete their Didactic Program in Dietetics coursework). Participants' ages ranged from 18 to 40 years old. Gender, racial/ethnic group, or disability was not an exclusionary factor. The final sample was $85 \%$ female, $15 \%$ male, with approximately $25 \%$ of the population being in an ethnic minority.

The initial sample consisted of a random selection of 113 students enrolled in at least one upper level course in nutrition or Didactic Program in Dietetics course throughout the State of Kentucky. The final sample consisted of 96 students who completed the entire survey. The proportion was $83 \%$ female, $17 \%$ male, and approximately $11 \%$ of the sample was in an ethnic minority. The average body mass index of the group was $22.42^{\mathrm{kg} / \mathrm{m}^{\wedge} 2}$, a value in the "normal" range, and the vast majority $(89 \%)$ of participants reported their dietary eating preferences as omnivorous. As intended by targeting upper level nutrition courses, all of the participants reported that they were in their junior (41\%) or senior (56\%) year in school; the remaining 3\% consisted of graduate or doctoral students - these students, though graduate/doctoral students, were enrolled in an upper level 
undergraduate nutrition course. Correspondingly, the reported ages matched the academic level: 18 - 20 year olds made up 24\% of the sample, and 21 - 23 year olds comprised $72 \%$; the remaining $4 \%$ were 24 years old and above.

The target population consisted of students enrolled in at least one upper level nutrition course; the two majors represented in this population were dietetics (a didactic program in dietetics - these are students who intend to become dietitians) and human nutrition (a pre-professional major - these students may pursue a career as a dietitian, or professional school, such as medical, dental, physical therapy, and physician's assistant). In the final sample, dietetics represented $70 \%(n=67)$ of the sample, while $30 \%(n=29)$ represented human nutrition. The differentiation has importance due to differences in academic focus within teaching content and course requirements; this difference will be further discussed in the conclusions section.

\section{Research Design}

The study was administered via Survey Monkey to nutrition students. Participants volunteered to take the survey. There was no control or experimental group, nor was deception involved. The majority of questions were designed through consultation of existing, valid food-behavior questionnaires (Blackburn et al, 2007; Parmenter, K., Wardle, J., 1999; Robles, Delma S., 2010), while the responses were based on a standard Likert-type scale. The questions regarding the Scope of Dietetics Practice were developed based on the information contained in the framework. After the initial e-mail message to the Didactic Program in Dietetics 
university directors, two follow-up messages were sent - one midway through the collection period and one during the final week of data collection. These e-mails contained the purpose of the study, the target population, the start and completion dates, and an incentive to participate in the study, as well as the link to the survey.

\section{Data Collection}

Data were collected through one online survey created in Survey Monkey ${ }^{\odot}$. Respondents who did not complete the entire survey were eliminated.

\section{Measurements}

Data were assessed to determine if the participant's knowledge and application of the Scope of Dietetics Practice or their major impacted their responses. The main portion of the survey concerned advice that the students could give to individuals and family members, situational cases requiring nutritional recommendations to others, and situational, stress-causing cases encountered by many students during their collegiate years. In addition, students responded to questions regarding their ability to appropriately model of food and nutrition behaviors and behave in a credible and reliable manner.

\section{Data Analysis}

Data were entered, managed, and analyzed using SAS (version 9.2, English). Data were divided into seven categories: solicited advice; unsolicited advice; situational cases requiring nutritional recommendations to family members and/or individuals; situational, stress-causing cases encountered by most college students; 
Scope of Dietetics Practice; modeling food and nutrition behaviors; and credibility and reliability behaviors.

To analyze the data, each of the individual questions within the categories listed above was compared to responses given to the questions regarding Scope of Dietetics Practice and the participant's major; this determined if the participants' comprehension and use of the Scope of Dietetics Practice and/or their major had any effect on the response they would have to the situation described in the individual questions. By evaluating any trends that appear, universities could modify their curriculum to better prepare all students studying nutrition, in a dietetics or human nutrition capacity, to function optimally within the confines of the Scope of Dietetics Practice. A chi-square test was used to determine if a relationship existed; chi-square tests have the ability to conclude if data have a relationship with different variables. Once the chi-square analysis was complete, the results were analyzed for overall trends in questions that carried significance. An alpha level of 0.05 or less was considered significant. 


\section{Chapter 4: Results}

\section{Demographics}

The final sample for this study consisted of ninety-six participants: eighty female participants (83\%) and sixteen male participants (17\%). Ethnic make-up for the sample was $89 \%$ Caucasian $(n=85), 11 \%$ ethnic minority $(n=11)$; the minorities represented include African American, Middle Eastern, and Asian/Pacific Islander. Only upper classmen were included in the study; $41 \%(n=39)$ of the participants reported that they were in their junior year, $56 \%(n=54)$ stated they were in their senior year, and 3\% $(n=3)$ indicated that they were at the graduate/doctoral level. Correspondingly, 24\% $(n=23)$ were in the $18-20$ year old range, $72 \%(n=69)$ reported being $21-23$ years old, $2 \%(n=2)$ were $24-30$, and $2 \%(n=2)$ were 31 years or older. As this is a nutrition-related study, dietary preference was asked as a part of the demographic questions. Eighty-nine percent $(n=85)$ reported an omnivorous eating style, while $11 \%(n=11)$ indicated "other;" the "other" preferences included vegetarian (no meat), fruitarian (fruits, nuts, and seeds), flexitarian (primarily vegetarian, but may incorporate meat on occasion), vegan (no animal products), and pescetarian (vegetarian with fish).

Because money serves as a source of stress for many people, participants were asked to indicate their household income and the primary source paying for their college tuition. Eleven percent reported that their household/family income was under $\$ 16,000,7 \%$ indicated that they were within the $\$ 16,000$ to $\$ 34,999$ range, $42 \%$ were in the $\$ 35,000$ to $\$ 99,999$ range, $34 \%$ in the $\$ 100,000$ to $\$ 499,999$ range, and $5 \%$ in households bringing in $\$ 500,000$ or more. The primary source of 
paying for college tuition were participants' parents (49\%); other than parents, the primary sources of college tuition were $25 \%$ FAFSA student loans, $6 \%$ savings, $16 \%$ scholarships, $1 \%$ personal employment, and 3\% "other."

One of the aspects of this study was the comparison of students studying human nutrition to those studying dietetics. Seventy percent $(n=67)$ of the study's participants majored in dietetics; 30\% $(n=29)$ majored in human nutrition. The average body mass index (BMI) was calculated from the participants' self-reported height and weight. BMI is calculated by weight (in pounds) divided by height (in inches squared), multiplied by 703 . The average BMI for the sample was $22.65 \mathrm{~kg} / \mathrm{m}^{\wedge} 2$; however, the average BMI for dietetics students was $21.9 \mathrm{~kg} / \mathrm{m}^{\wedge} 2$ and the average BMI for Human Nutrition students was $24.3 \mathrm{~kg} / \mathrm{m}^{\wedge} 2$.

Table 1 below illustrates the demographic make-up of the sample. 
Table 1: Demographics

\begin{tabular}{|c|c|c|c|}
\hline \multicolumn{4}{|l|}{ Sample Characteristics $(\mathrm{n}=96)$} \\
\hline & & Number & Percent \\
\hline \multirow{2}{*}{ Gender } & Female & 80 & $83 \%$ \\
\hline & Male & 16 & $17 \%$ \\
\hline \multirow{4}{*}{ Ethnicity } & African American & 8 & $8 \%$ \\
\hline & Asian/Pacific Islander & 2 & $2 \%$ \\
\hline & Caucasian & 85 & $89 \%$ \\
\hline & Middle Eastern & 1 & $1 \%$ \\
\hline \multirow{2}{*}{ Major } & Dietetics & 67 & $70 \%$ \\
\hline & Human Nutrition & 29 & $30 \%$ \\
\hline \multirow{3}{*}{ Year in School } & Junior & 39 & $41 \%$ \\
\hline & Senior & 54 & $56 \%$ \\
\hline & Graduate/Doctoral & 3 & $3 \%$ \\
\hline \multirow{4}{*}{ Age } & $18-20$ years old & 23 & $24 \%$ \\
\hline & 21-23 years old & 69 & $72 \%$ \\
\hline & 24-30 years old & 2 & $2 \%$ \\
\hline & $31+$ years old & 2 & $2 \%$ \\
\hline \multirow{3}{*}{ Average Body Mass Index (BMI) } & Sample Average & \multicolumn{2}{|c|}{$22.65 \mathrm{~kg} / \mathrm{m}^{\wedge} 2$} \\
\hline & Dietetics & \multicolumn{2}{|r|}{$21.9 \mathrm{~kg} / \mathrm{m}^{\wedge} 2$} \\
\hline & Human Nutrition & \multicolumn{2}{|r|}{$24.3^{\mathrm{kg} / \mathrm{m}^{\wedge} 2}$} \\
\hline \multirow{6}{*}{ Dietary Preference } & Flexitarian & 1 & $1 \%$ \\
\hline & Fruitarian & 1 & $1 \%$ \\
\hline & Omnivore & 85 & $89 \%$ \\
\hline & Pescetarian & 1 & $1 \%$ \\
\hline & Vegan & 2 & $2 \%$ \\
\hline & Vegetarian & 6 & $6 \%$ \\
\hline \multirow{5}{*}{ Household Income } & Less than $\$ 16,000$ & 11 & $11 \%$ \\
\hline & $\$ 16,000-\$ 34,999$ & 7 & $7 \%$ \\
\hline & $\$ 35,000-\$ 99,999$ & 40 & $42 \%$ \\
\hline & $\$ 100,000-\$ 499,999$ & 33 & $34 \%$ \\
\hline & $\$ 500,000$ or more & 5 & $5 \%$ \\
\hline \multirow{6}{*}{ Primary Source of College Tuition } & FAFSA Student Loans & 24 & $25 \%$ \\
\hline & Parents & 47 & $49 \%$ \\
\hline & Personal Employment & 1 & $1 \%$ \\
\hline & Other & 3 & $3 \%$ \\
\hline & Savings & 6 & $6 \%$ \\
\hline & Scholarship & 15 & $16 \%$ \\
\hline
\end{tabular}


Significant Correlations Regarding Scope of Dietetics Practice:

The Scope of Dietetics Practice, as defined by the American Dietetic Association, indicates expectations, responsibilities, and duties for nutrition professionals of all levels. It is the obligation of all levels of nutrition professionals to know what they are ethically bound to do or avoid. The five statements below were developed for this survey to evaluate the participants' knowledge and comprehension of the Scope of Practice using a Likert-type scale with participants selecting one of the following words or phrases: "strongly disagree," "disagree," "neutral," "agree," and "strongly agree." In addition, these statements were compared individually to the other portions of the survey and analyzed to determine if the participants' knowledge and comprehension correlated with their responses to the other questions.

1. "I only deliver information allowed at the student level by the CDR, as specified in the Scope of Dietetics Practice."

A five point Likert-type scale was used and the significance level was $\mathrm{p}<0.05$. The five points on the scale were "strongly disagree," "disagree," "neutral," "agree," and "strongly agree." Zero percent $(\mathrm{n}=0)$ of the sample responded "strongly disagree," 4\% (n=4) responded "disagree," 51\% (n=48) responded "neutral," 35\% $(n=33)$ responded "agree," and 11\% $(n=10)$ responded "strongly agree."

Students who reported that they were more likely to only deliver information allowed at the student level by the Commission on Dietetics 
Registration (CDR), as specified in the Scope of Dietetics Practice were more likely

to do the following:

- Recommend consumption of smaller, more frequent meals instead of three large meals to an individual who is overweight/obese and trying to attain a healthy weight $(\mathrm{p}=0.01)$;

- Recommend avoiding calorically dense foods and beverages to an individual who is overweight/obese and trying to attain a healthy weight $(p<0.001)$;

- Recommend eating smaller meals throughout the day instead of three large meals to an individual trying to avoid the "Freshman 15" ( $\mathrm{p}=0.01)$;

- Recommend a high protein, high calorie diet to someone who is underweight and wants to achieve a healthy weight $(\mathrm{p}=0.02)$;

- Immerse themselves in their schoolwork when coping with the stress of unexpected expenses $(\mathrm{p}=0.01)$.

Participants who reported that they were more likely to only deliver information allowed at the student level by the Commission on Dietetics Registration (CDR), as specified in the Scope of Dietetics Practice were less likely to do the following:

- Give unsolicited advice recommending 60 minutes of exercise most days of the week $(\mathrm{p}<0.001)$;

- Give unsolicited advice recommending not eating or drinking anything after dinner $(\mathrm{p}=0.05)$;

- Recommend at least 60 minutes of exercise most days of the week to an individual who is overweight/obese and trying to attain a healthy weight $(\mathrm{p}=0.03)$;

- Recommend at least 60 minutes of exercise, most days of the week to an individual trying to avoid the "Freshman 15" ( $\mathrm{p}<0.001)$;

- Recommend consuming less alcoholic beverages to an individual trying to avoid the "Freshman 15" ( $\mathrm{p}=0.04)$; 
- Recommend avoidance of eating or drinking anything after dinner to someone who is underweight and wants to achieve a healthy weight $(\mathrm{p}=0.05)$;

- Consume alcoholic beverages when coping with the stress of ending a relationship with their significant other $(\mathrm{p}=0.03)$;

- Consume alcoholic beverages when coping with the stress of unexpected expenses $(\mathrm{p}<0.001)$;

- Manage the stress of maintaining a certain GPA through exercise $(p=0.03)$

- Consume alcoholic beverages to cope with the stress of maintaining a certain GPA $(\mathrm{p}<0.001)$;

- Exercise to cope with the stress of difficult exams $(\mathrm{p}=0.01)$.

The figure below (Figure 1) illustrates the differences between dietetics majors and human nutrition majors.

Figure 1: Scope of Dietetics Practice \#1

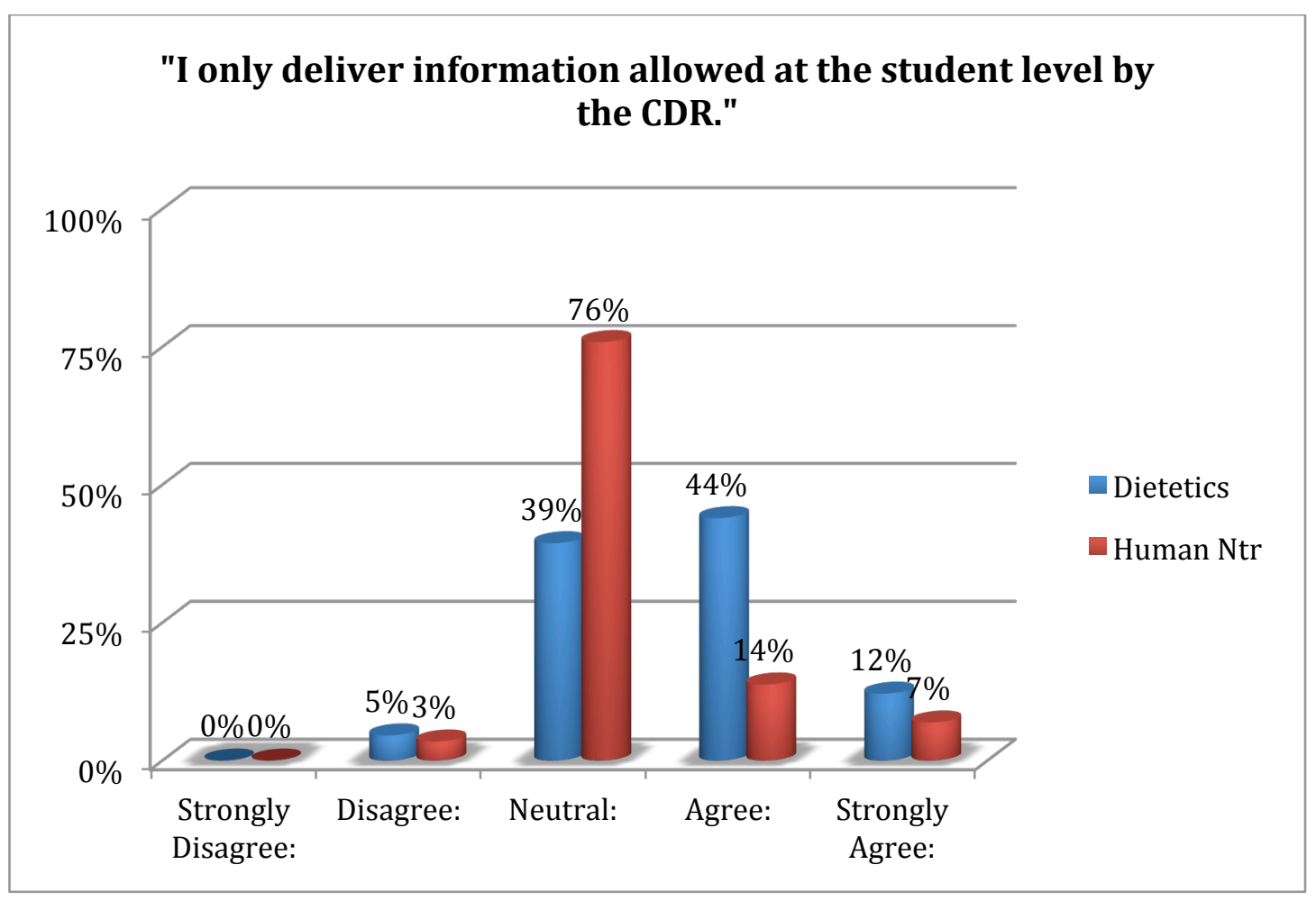


2. "I comprehend the scope of practice."

A five point Likert-type scale was used and the significance level was $\mathrm{p}<0.05$. The five points on the scale were "strongly disagree," "disagree," "neutral," "agree," and "strongly agree." One percent $(n=1)$ of the sample responded "strongly disagree," 6\% $(n=6)$ responded "disagree," 33\% (n=32) responded "neutral," 46\% (n=44) responded "agree," and 14\% (n=13) responded "strongly agree." The significance level was $\mathrm{p}<0.05$.

Students who reported a better comprehension of the Scope of Dietetics Practice were more likely to do the following:

- Give solicited advice to get at least 60 minutes of exercise per day, most days of the week $(\mathrm{p}=0.01)$;

- Give unsolicited advice recommending 60 minutes of exercise most days of the week $(p=0.01)$;

- Recommend at least 60 minutes of exercise most days of the week to an individual who is overweight/obese and trying to attain a healthy weight $(\mathrm{p}=0.03)$;

- Recommend a high protein, high calorie diet to an individual who is overweight/obese and trying to attain a healthy weight ( $p=0.02)$;

- Recommend at least 60 minutes of exercise, most days of the week to an individual trying to avoid the "Freshman 15 " ( $p=0.04)$;

- Recommend making smart decisions when eating away from home for someone trying to avoid the "Freshman 15 " ( $\mathrm{p}=0.02)$;

- Recommend getting at least 60 minutes of exercise most days of the week to someone who is underweight and wants to achieve a healthy weight $(\mathrm{p}=0.04)$;

- Recommend avoidance of eating or drinking anything after dinner to someone who is underweight and wants to achieve a healthy weight $(\mathrm{p}=0.04)$; 
- Encourage friends and family to read nutrition labels while grocery shopping ( $\mathrm{p}=0.05)$;

- Believe that the information they give to others is usable, practical, and accurate $(\mathrm{p}=0.01)$;

- Believe that their height and weight support their credibility ( $\mathrm{p}=0.01)$. Students who reported a better comprehension of the Scope of Dietetics Practice were less likely to do the following:

- Recommend avoiding calorically dense foods and beverages to an individual who is overweight/obese and trying to attain a healthy weight ( $p<0.001)$;

- Recommend a high protein, high calorie diet to someone who is underweight and wants to achieve a healthy weight $(\mathrm{p}=0.02)$;

- Recommend avoidance of calorically dense foods and beverages to someone who is underweight and wants to achieve a healthy weight ( $\mathrm{p}=0.02)$;

- Recommend consuming fewer alcoholic beverages to someone who is underweight and wants to achieve a healthy weight $(\mathrm{p}=0.03)$;

- Immerse themself in schoolwork when coping with the stress of ending a relationship with their significant other $(\mathrm{p}=0.01)$;

- Eat their favorite junk food when coping with the stress of unexpected expenses $(\mathrm{p}=0.02)$;

- Cope with the stress of the death of a family or friend by eating their favorite junk food ( $\mathrm{p}=0.03)$;

- Cope with the stress of maintaining a certain GPA by eating their favorite junk food $(\mathrm{p}=0.01)$;

- Consume their favorite junk food to cope with the stress of irritating roommates/neighbors $(\mathrm{p}=0.04)$.

The figure below (Figure 2) illustrates the differences between dietetics majors and human nutrition majors. 
Figure 2: Scope of Dietetics Practice \#2

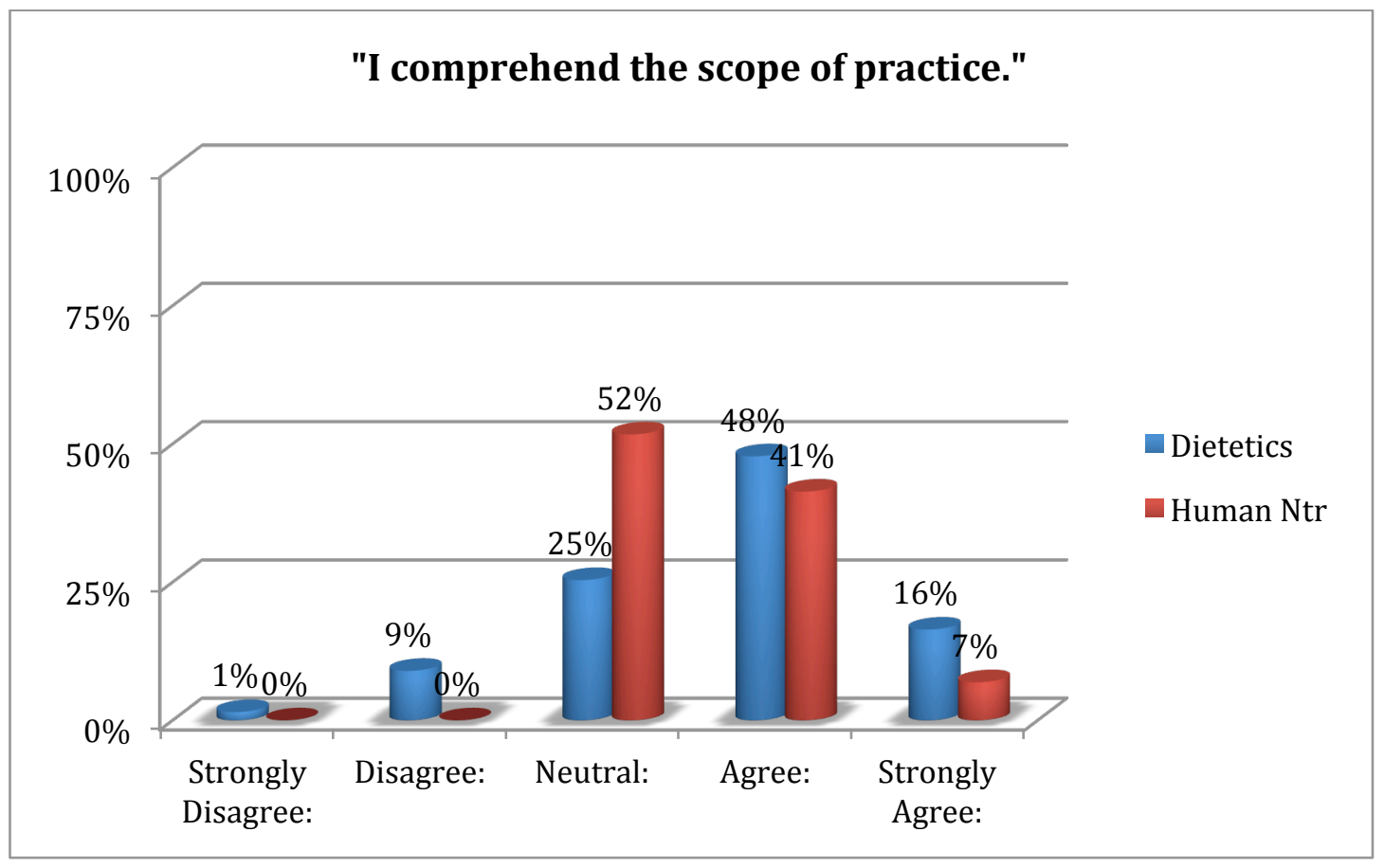

3. "I refer friends and family to other sources that were outside my scope of practice."

A five point Likert-type scale was used and the significance level was $\mathrm{p}<0.05$. The five points on the scale were "strongly disagree," "disagree," "neutral," "agree," and "strongly agree." One percent $(\mathrm{n}=1)$ of the sample responded "strongly disagree," $2 \%(n=2)$ responded "disagree," 21\% (n=20) responded "neutral," 54\% (n=52) responded "agree," and 22\% (n=21) responded "strongly agree." The significance level was $\mathrm{p}<0.05$.

Students who reported that they were more likely to refer friends and family to other sources for information outside their scope of practice as a student were more likely to do the following: 
- Recommend avoiding calorically dense foods and beverages to an individual who is overweight/obese and trying to attain a healthy weight $(\mathrm{p}=0.01)$;

- Recommend consuming less alcoholic beverages to an individual trying to avoid the "Freshman 15" ( $\mathrm{p}=0.01)$;

- Recommend eating meals throughout the day instead of three large meals for someone who is underweight and trying to achieve a healthy weight $(\mathrm{p}=0.02)$;

- Exercise to cope with the stress of the death of a friend or family member $(\mathrm{p}=0.02)$;

- Manage the stress of maintaining a certain GPA by calling a friend or family member $(\mathrm{p}=0.02)$;

- Manage the stress of maintaining a certain GPA through exercise $(p=0.04)$;

- Exercise to cope with the stress of difficult exams $(\mathrm{p}<0.01)$;

- Consume alcoholic beverages to cope with the stress of irritating roommates/neighbors $(\mathrm{p}=0.03)$;

- Model correct portion sizes when eating with friends and family $(\mathrm{p}<0.01)$;

- Believe that the information they give to others is usable, practical, and accurate $(\mathrm{p}=0.01)$;

- Believe that their friends and family find their information reliable based on their year in school $(\mathrm{p}=0.03)$;

- Believe that their height and weight support their credibility ( $\mathrm{p}=0.01)$;

- Incorporate high fiber, low fat foods into their diet on a regular basis $(\mathrm{p}<0.01)$.

Students who reported that they were more likely to refer friends and family to other sources for information outside their scope of practice as a student were more likely to do the following:

- Give unsolicited advice recommending a high protein, low carbohydrate diet $(\mathrm{p}=0.01)$; 
- Give unsolicited advice recommending a high protein, high calorie diet $(\mathrm{p}=0.01)$;

- Recommend a high protein, high calorie diet to an individual who is overweight/obese and trying to attain a healthy weight ( $\mathrm{p}=0.01)$;

- Recommend a high protein, high calorie diet to an individual trying to avoid the "Freshman 15" ( $\mathrm{p}=0.05)$;

- Recommend consuming fewer alcoholic beverages to someone who is underweight and wants to achieve a healthy weight ( $\mathrm{p}=0.01)$;

- Manage the stress of irritating roommates/neighbors by exercising $(\mathrm{p}=0.01)$.

The figure below (Figure 3) illustrates the differences between dietetics majors and human nutrition majors.

Figure 3: Scope of Dietetics Practice \#3

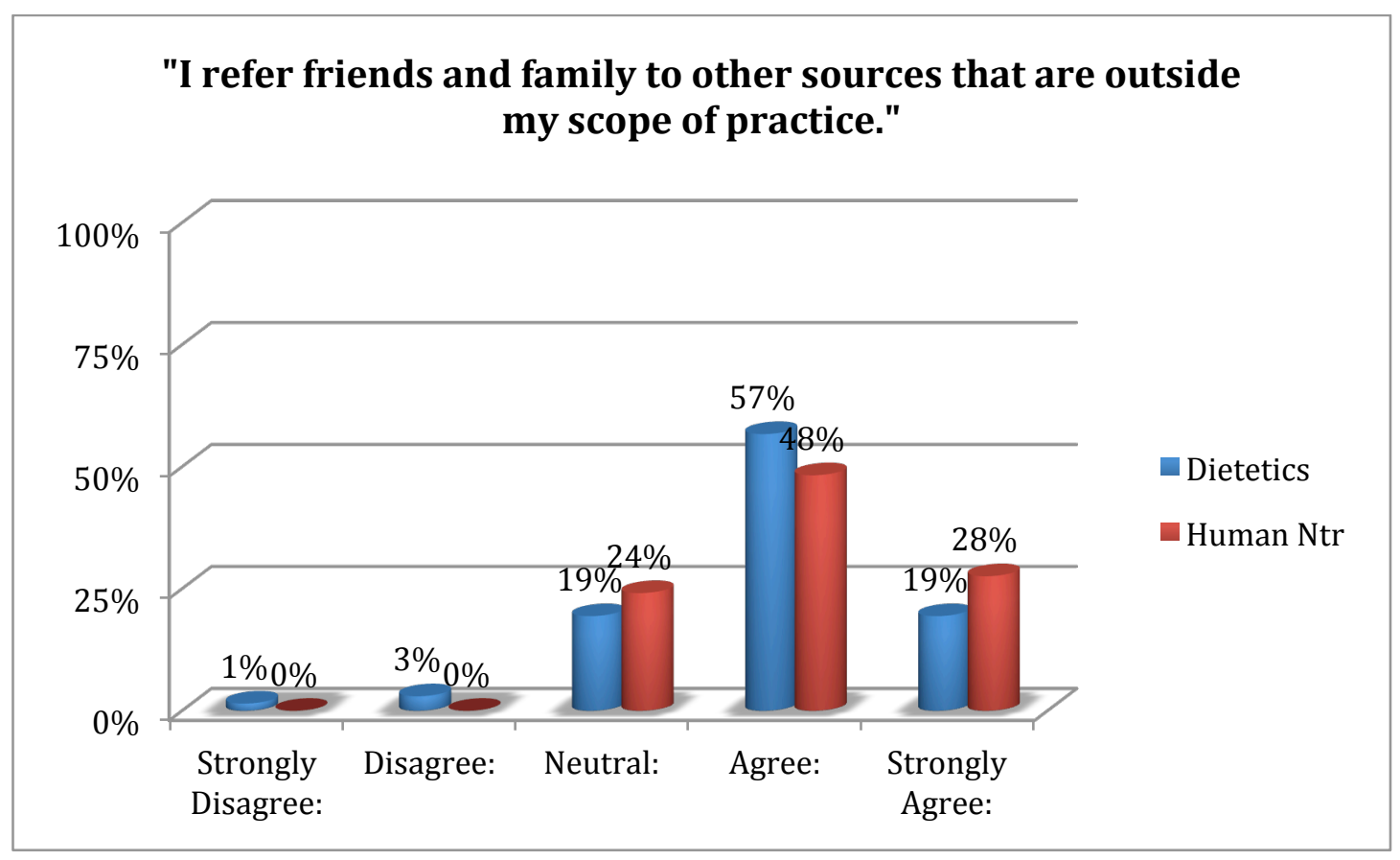


4. "I abide by the Dietetics Code of Ethics."

A five point Likert-type scale was used and the significance level was $\mathrm{p}<0.05$. The five points on the scale were "strongly disagree," "disagree," "neutral," "agree," and "strongly agree." Zero percent $(\mathrm{n}=0)$ of the sample responded "strongly disagree," 2\% (n=2) responded "disagree," 24\% (n=23) responded "neutral," 50\% ( $n=48)$ responded "agree," and 24\% (n=24) responded "strongly agree."

Students who reported that they abided by the code of ethics were more likely to abide by the Dietetics Code of Ethics were more likely to do the following:

- Give solicited advice to get at least 60 minutes of exercise per day, most days of the week ( $\mathrm{p}=0.01)$;

- Give solicited advice to use the Internet as a resource for nutrition facts at restaurants ( $\mathrm{p}=0.05)$;

- Give unsolicited advice recommending 60 minutes of exercise most days of the week $(\mathrm{p}<0.01)$;

- Give unsolicited advice recommending not eating or drinking anything after dinner $(\mathrm{p}=0.02)$;

- Give unsolicited advice recommending a high protein, high calorie diet $(\mathrm{p}=0.02)$;

- Give unsolicited advice recommending consumption of fewer alcoholic beverages $(\mathrm{p}<0.001)$;

- Recommend at least 60 minutes of exercise most days of the week to an individual who is overweight/obese and trying to attain a healthy weight $(\mathrm{p}=0.03)$;

- Eat their favorite junk food when coping with the stress of ending a relationship with their significant other $(\mathrm{p}<0.01)$;

- Cope with the stress of the death of a family or friend by eating their favorite junk food ( $\mathrm{p}=0.02)$;

- Manage the stress of maintaining a certain GPA through exercise $(\mathrm{p}=0.05)$; 
- Immerse themselves in school work when faced with a great deal of difficult exams in a short amount of time $(\mathrm{p}=0.05)$.

Participants who reported that they abided by the Dietetics Code of Ethics were less likely to do the following:

- Recommend consumption of smaller, more frequent meals instead of three large meals to an individual who is overweight/obese and trying to attain a healthy weight $(\mathrm{p}=0.02)$;

- Recommend at least 60 minutes of exercise, most days of the week to an individual trying to avoid the "Freshman 15 " ( $\mathrm{p}<0.01)$;

- Recommend a high protein, high calorie diet to an individual trying to avoid the "Freshman 15" ( $\mathrm{p}=0.04)$;

- Recommend eating meals throughout the day instead of three large meals for someone who is underweight and trying to achieve a healthy weight $(\mathrm{p}=0.02)$;

- Immerse themselves in schoolwork when coping with the stress of ending a relationship with their significant other $(\mathrm{p}=0.01)$;

- Immerse themselves in their schoolwork when coping with the stress of unexpected expenses $(\mathrm{p}=0.03)$;

- Cope with the stress of maintaining a certain GPA by eating their favorite junk food $(p=0.05)$.

The figure below (Figure 4) illustrates the differences between dietetics majors and human nutrition majors. 
Figure 4: Scope of Dietetics Practice \#4

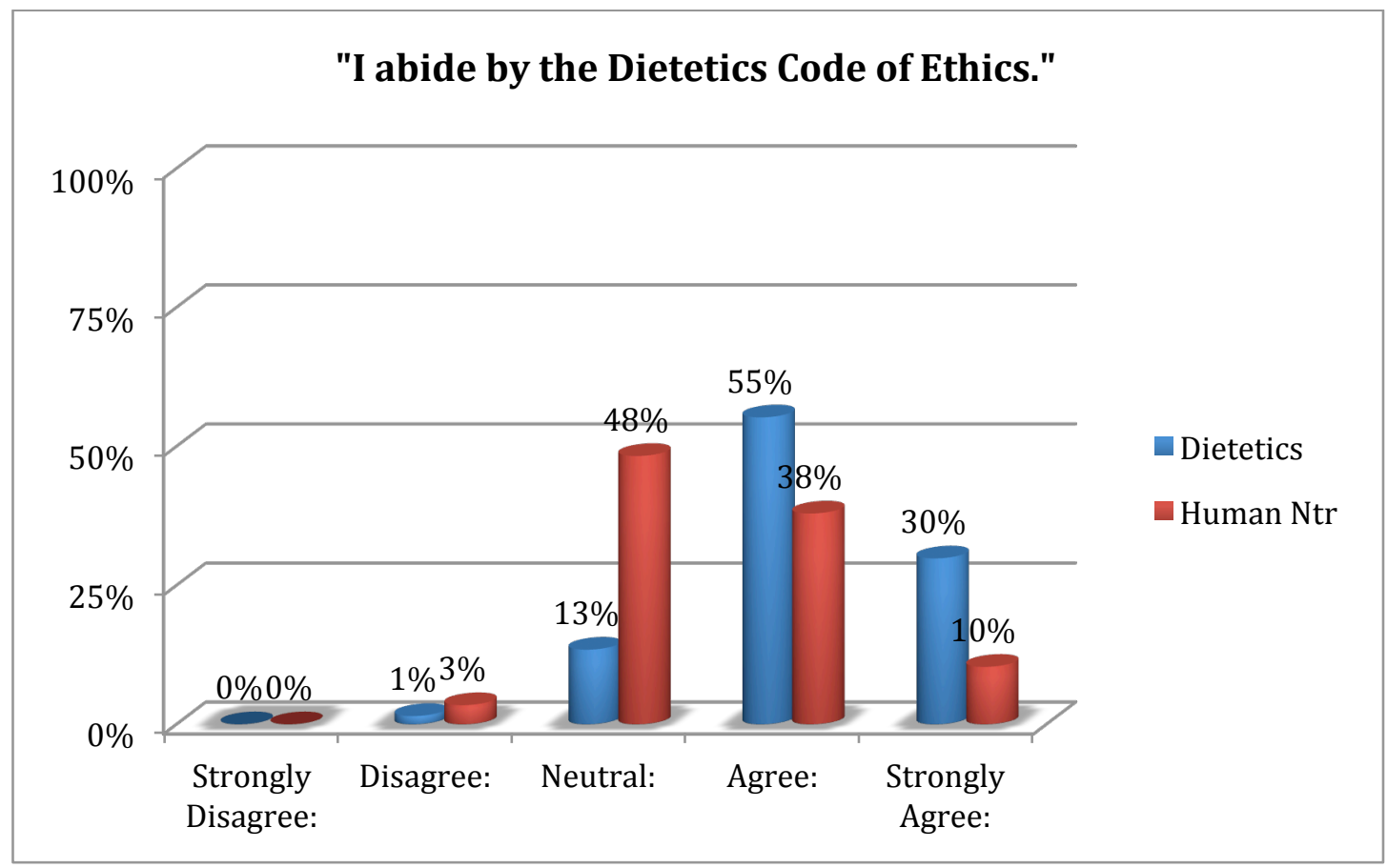

5. "I use nutrition information to diagnose health conditions in family members and individuals."

A five point Likert-type scale was used and the significance level was $\mathrm{p}<0.05$. The five points on the scale were "strongly disagree," "disagree," "neutral," "agree," and "strongly agree." Eight percent $(\mathrm{n}=8)$ of the sample responded "strongly disagree," 24\% (n=23) responded "disagree," 30\% (n=29) responded "neutral," 27\% $(\mathrm{n}=26)$ responded "agree," and 10\% $(\mathrm{n}=10)$ responded "strongly agree."

Participants who reported they were more likely to use nutrition information to diagnose health conditions in family members and individuals were more likely to do the following:

- Give solicited advice to eat a high protein, low carbohydrate diet $(\mathrm{p}<0.001)$; 
- Give solicited advice recommending a high protein, high calorie diet $(\mathrm{p}<0.001)$

- Give unsolicited advice recommending a high protein, low carbohydrate diet $(\mathrm{p}<0.001)$;

- Give unsolicited advice recommending consumption of several smaller meals throughout the day instead of three large meals $(\mathrm{p}=0.02)$;

- Give unsolicited advice recommending not eating or drinking anything after dinner $(\mathrm{p}=0.01)$;

- Give unsolicited advice recommending avoidance of calorically dense foods and beverages $(\mathrm{p}=0.02)$;

- Give unsolicited advice recommending consumption of fewer alcoholic beverages $(\mathrm{p}=0.01)$;

- Recommend avoiding anything to eat or drink after dinner to an individual who is overweight/obese and trying to attain a healthy weight $(\mathrm{p}<0.01)$;

- Recommend a high protein, low carbohydrate diet to an individual trying to avoid the "Freshman 15" ( $\mathrm{p}=0.02)$;

- Recommend a high protein, high calorie diet to an individual trying to avoid the "Freshman 15" ( $\mathrm{p}=0.02)$;

- Recommend a high protein, low carbohydrate diet to someone who is underweight and wants to gain weight $(\mathrm{p}=0.05)$;

- Recommend avoidance of eating or drinking anything after dinner to someone who is underweight and wants to achieve a healthy weight $(\mathrm{p}=0.05)$;

- Eat their favorite junk food when coping with the stress of ending a relationship with their significant other $(\mathrm{p}=0.01)$;

- Cope with the stress of the death of a family or friend by eating their favorite junk food $(\mathrm{p}<0.01)$;

- Consume their favorite junk food to cope with the stress of irritating roommates/neighbors $(\mathrm{p}=0.02)$;

- Eat junk food when faced with midterms/finals-related stress ( $\mathrm{p}=0.02)$; 
- Follow dietary guidelines regarding alcohol intake $(\mathrm{p}=0.02)$.

Participants who reported that they were more likely to use nutrition information to diagnose health conditions in family members and individuals were less likely to do the following:

- Give unsolicited advice recommending a high protein, high calorie diet $(\mathrm{p}<0.001)$;

- Recommend a high protein, low carbohydrate diet to an individual who is overweight/obese and trying to attain a healthy weight $(\mathrm{p}<0.001)$;

- Recommend at least 60 minutes of exercise most days of the week to an individual who is overweight/obese and trying to attain a healthy weight $(\mathrm{p}<0.001)$;

- Recommend a high protein, high calorie diet to an individual who is overweight/obese and trying to attain a healthy weight $(\mathrm{p}=0.03)$;

- Recommend making smart decisions when eating away from home for someone trying to avoid the "Freshman 15 " ( $\mathrm{p}=0.02)$;

- Call a friend/family member to cope with the stress of difficult exams $(\mathrm{p}=0.02)$.

The figure below (Figure 5) illustrates the differences between dietetics majors and human nutrition majors. 
Figure 5: Scope of Dietetics Practice \#5

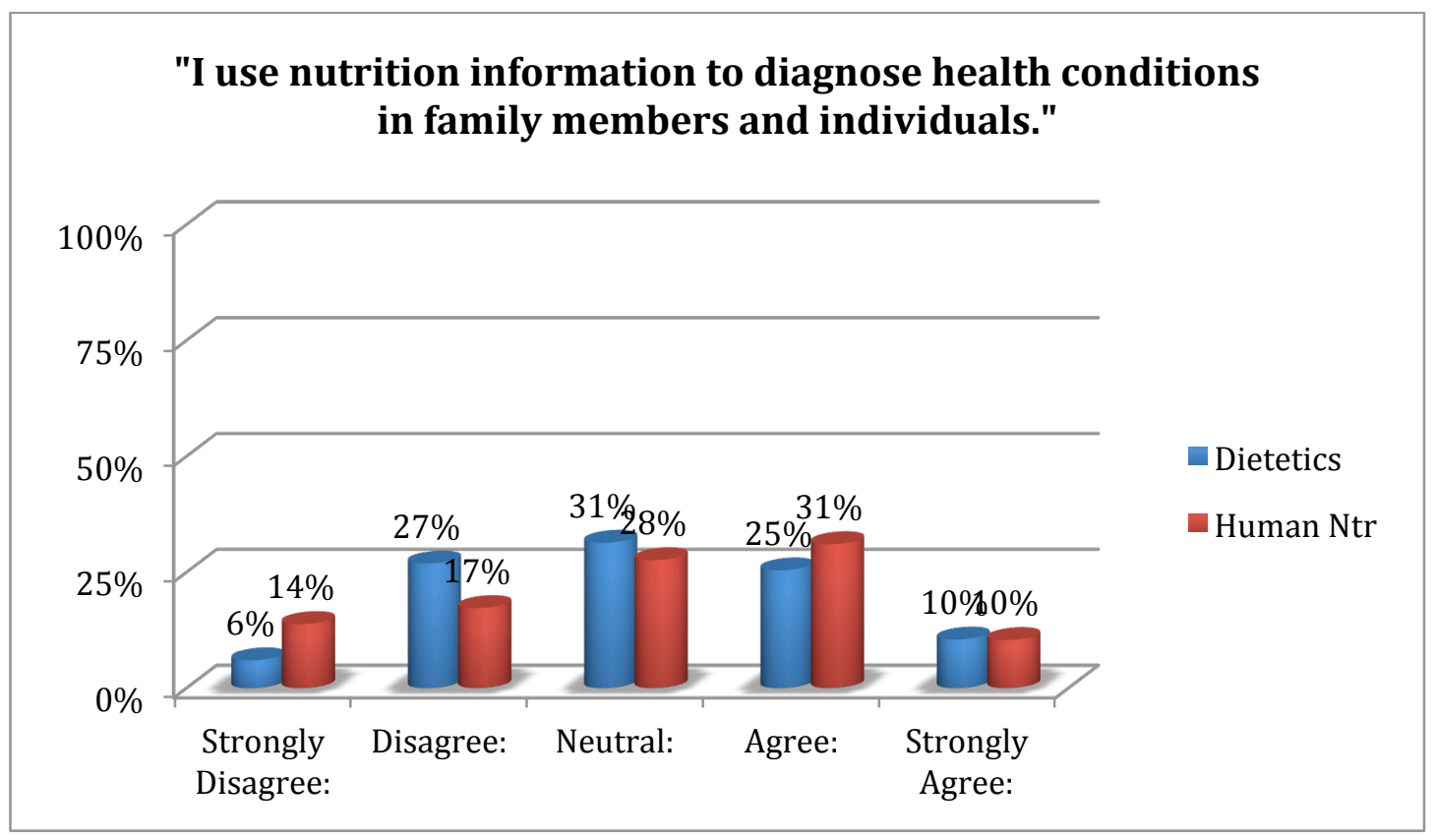

Significant Correlations Involving Course of Study

A five point Likert-type scale was used and the significance level was $\mathrm{p}<0.05$. The five points on the scale were "strongly disagree," "disagree," "neutral," "agree," and "strongly agree." Participants majoring in dietetics were more likely to do the following, as compared to the participants studying human nutrition:

- Recommend making smart food decisions when eating away from home to an individual who is overweight/obese and trying to attain a healthy weight $(\mathrm{p}=0.04)$;

- Recommend using the Internet as a resource for nutrition facts at restaurants to an individual who is overweight/obese and trying to attain a healthy weight $(\mathrm{p}=0.04)$;

- Recommend avoidance of calorically dense foods and beverages to someone who is underweight and wants to achieve a healthy weight ( $\mathrm{p}=0.02)$;

- Recommend consuming fewer alcoholic beverages to someone who is underweight and wants to achieve a healthy weight $(\mathrm{p}<0.001)$; 
- Believe that their height and weight supports their credibility ( $\mathrm{p}=0.01)$;

- Believe that their health behaviors (eating and exercise) support their credibility with family and individuals $(\mathrm{p}=0.01)$;

- Incorporate high fiber, low fat foods into their diet on a regular basis, $(\mathrm{p}=0.04)$.

Participants majoring in human nutrition were more likely to do the

following, as compared to the participants studying dietetics:

- Believe they modeled correct portion sizes when eating with friends and family as compared to dietetics majors $(\mathrm{p}<0.001)$;

- Immerse themselves in schoolwork to cope with the stress of the death of a friend or family member $(\mathrm{p}=0.04)$;

- Eat their favorite junk food when coping with the stress of ending a relationship with their significant other $(\mathrm{p}=0.03)$;

- Recommend at least 60 minutes of exercise, most days of the week to an individual trying to avoid the "Freshman 15" ( $\mathrm{p}<0.01)$.

The table (Table 2) below illustrates the overall results of the questions concerning the Scope of Dietetics Practice. Tables 3 and 4 show the specific breakdown for solicited and unsolicited questions, respectively, between dietetics participants and human nutrition participants; the accompanying figures (Figures 6-9) depict the highlights from the tables. Figure 10 depicts that human nutrition participants were more likely to consume junk food when coping with the stress of ending a relationship; it was the only stressor to carry significance between majors utilizing a food-related behavior. The remaining figures are located in Appendix E. 
Table 2: Scope of Practice

\begin{tabular}{|c|c|c|c|c|}
\hline \multicolumn{5}{|l|}{ Scope of Dietetics Practice } \\
\hline \multirow{2}{*}{$\begin{array}{l}\text { Question } \\
\text { 1. I only deliver information } \\
\text { allowed at the student level by } \\
\text { the CDR. }\end{array}$} & \multicolumn{2}{|l|}{ Dietetics } & \multicolumn{2}{|c|}{ Human Nutrition } \\
\hline & Number & Percentage & Number & Percentage \\
\hline Strongly Disagree & 0 & $0 \%$ & 0 & $0 \%$ \\
\hline Disagree & 3 & $5 \%$ & 1 & $3 \%$ \\
\hline Neutral & 26 & $39 \%$ & 22 & $76 \%$ \\
\hline Agree & 29 & $44 \%$ & 4 & $14 \%$ \\
\hline Strongly Agree & 8 & $12 \%$ & 2 & $7 \%$ \\
\hline $\begin{array}{l}\text { 2. I comprehend the scope of } \\
\text { practice. }\end{array}$ & Number & Percentage & Number & Percentage \\
\hline Strongly Disagree & 1 & $1 \%$ & 0 & $0 \%$ \\
\hline Disagree & 6 & $9 \%$ & 0 & $0 \%$ \\
\hline Neutral & 17 & $25 \%$ & 15 & $52 \%$ \\
\hline Agree & 32 & $48 \%$ & 12 & $41 \%$ \\
\hline Strongly Agree & 11 & $16 \%$ & 2 & $7 \%$ \\
\hline $\begin{array}{l}\text { 3. I refer friends and family to } \\
\text { other sources that are outside my } \\
\text { scope of practice. }\end{array}$ & Number & Percentage & Number & Percentage \\
\hline Strongly Disagree & 1 & $1 \%$ & 0 & $0 \%$ \\
\hline Disagree & 2 & $3 \%$ & 0 & $0 \%$ \\
\hline Neutral & 13 & $19 \%$ & 7 & $24 \%$ \\
\hline Agree & 38 & $57 \%$ & 14 & $48 \%$ \\
\hline Strongly Agree & 13 & $19 \%$ & 8 & $28 \%$ \\
\hline $\begin{array}{l}\text { 4. I abide by the Dietetics Code of } \\
\text { Ethics }\end{array}$ & Number & Percentage & Number & Percentage \\
\hline Strongly Disagree & 0 & $0 \%$ & 0 & $0 \%$ \\
\hline Disagree & 1 & $1 \%$ & 1 & $3 \%$ \\
\hline Neutral & 9 & $13 \%$ & 14 & $48 \%$ \\
\hline Agree & 37 & $55 \%$ & 11 & $38 \%$ \\
\hline Strongly Agree & 20 & $30 \%$ & 3 & $10 \%$ \\
\hline $\begin{array}{l}\text { 5. I use nutrition information to } \\
\text { diagnose health conditions in } \\
\text { family members and individuals. }\end{array}$ & Number & Percentage & Number & Percentage \\
\hline Strongly Disagree & 4 & $6 \%$ & 4 & $14 \%$ \\
\hline Disagree & 18 & $27 \%$ & 5 & $17 \%$ \\
\hline Neutral & 21 & $31 \%$ & 8 & $28 \%$ \\
\hline Agree & 17 & $25 \%$ & 9 & $31 \%$ \\
\hline Strongly Agree & 7 & $10 \%$ & 3 & $10 \%$ \\
\hline
\end{tabular}


Table 3 (part A): Solicited Advice

\begin{tabular}{|c|c|c|c|c|}
\hline \multicolumn{5}{|l|}{ Solicited Advice } \\
\hline \multirow{2}{*}{\begin{tabular}{|l|} 
Question \\
$\begin{array}{l}\text { Eat a high protein, low } \\
\text { carbohydrate diet }\end{array}$ \\
\end{tabular}} & \multicolumn{2}{|l|}{ Dietetics } & \multicolumn{2}{|c|}{ Human Nutrition } \\
\hline & Number & Percentage & Number & Percentage \\
\hline Never & 25 & $38 \%$ & 16 & $55 \%$ \\
\hline Rarely & 18 & $27 \%$ & 5 & $17 \%$ \\
\hline Sometimes & 15 & $23 \%$ & 8 & $28 \%$ \\
\hline Often & 7 & $11 \%$ & 0 & $0 \%$ \\
\hline Always & 1 & $2 \%$ & 0 & $0 \%$ \\
\hline $\begin{array}{l}\text { Get at least } 60 \text { minutes of } \\
\text { exercise, most days of the week }\end{array}$ & Number & Percentage & Number & Percentage \\
\hline Never & 2 & $3 \%$ & 3 & $10 \%$ \\
\hline Rarely & 8 & $12 \%$ & 2 & $7 \%$ \\
\hline Sometimes & 21 & $31 \%$ & 6 & $21 \%$ \\
\hline Often & 22 & $33 \%$ & 12 & $41 \%$ \\
\hline Always & 14 & $21 \%$ & 6 & $21 \%$ \\
\hline $\begin{array}{l}\text { Eat several smaller meals } \\
\text { throughout the day instead of } 3 \\
\text { large meals }\end{array}$ & Number & Percentage & Number & Percentage \\
\hline Never & 2 & $3 \%$ & 2 & $7 \%$ \\
\hline Rarely & 8 & $12 \%$ & 2 & $7 \%$ \\
\hline Sometimes & 14 & $21 \%$ & 8 & $28 \&$ \\
\hline Often & 26 & $39 \%$ & 12 & $41 \%$ \\
\hline Always & 16 & $24 \%$ & 5 & $17 \%$ \\
\hline $\begin{array}{l}\text { Avoid eating or drinking anything } \\
\text { after dinner }\end{array}$ & Number & Percentage & Number & Percentage \\
\hline Never & 24 & $36 \%$ & 12 & $41 \%$ \\
\hline Rarely & 16 & $24 \%$ & 7 & $24 \%$ \\
\hline Sometimes & 17 & $25 \%$ & 6 & $21 \%$ \\
\hline Often & 8 & $12 \%$ & 4 & $14 \%$ \\
\hline Always & 2 & $3 \%$ & 0 & $0 \%$ \\
\hline $\begin{array}{l}\text { Eat a high protein, high calorie } \\
\text { diet }\end{array}$ & Number & Percentage & Number & Percentage \\
\hline Never & 35 & $52 \%$ & 18 & $62 \%$ \\
\hline Rarely & 26 & $39 \%$ & 7 & $24 \%$ \\
\hline Sometimes & 3 & $4 \%$ & 4 & $14 \%$ \\
\hline Often & 2 & $3 \%$ & 0 & $0 \%$ \\
\hline Always & 1 & $1 \%$ & 0 & $0 \%$ \\
\hline
\end{tabular}


Table 3 (part B): Solicited Advice

\begin{tabular}{|c|c|c|c|c|}
\hline \multicolumn{5}{|l|}{ Solicited Advice } \\
\hline \multirow{2}{*}{$\begin{array}{l}\text { Question } \\
\text { Avoid calorically dense foods and } \\
\text { beverages }\end{array}$} & \multicolumn{2}{|l|}{ Dietetics } & \multicolumn{2}{|c|}{ Human Nutrition } \\
\hline & Number & Percentage & Number & Percentage \\
\hline Never & 0 & $0 \%$ & 1 & $3 \%$ \\
\hline Rarely & 6 & $9 \%$ & 3 & $10 \%$ \\
\hline Sometimes & 28 & $42 \%$ & 13 & $45 \%$ \\
\hline Often & 28 & $42 \%$ & 9 & $31 \%$ \\
\hline Always & 5 & $7 \%$ & 3 & $10 \%$ \\
\hline Consume less alcoholic beverages & Number & Percentage & Number & Percentage \\
\hline Never & 3 & $4 \%$ & 8 & $28 \%$ \\
\hline Rarely & 13 & $19 \%$ & 4 & $14 \%$ \\
\hline Sometimes & 25 & $37 \%$ & 8 & $28 \%$ \\
\hline Often & 21 & $31 \%$ & 7 & $24 \%$ \\
\hline Always & 5 & $7 \%$ & 2 & $7 \%$ \\
\hline $\begin{array}{l}\text { Make smart food decisions when } \\
\text { eating away from home }\end{array}$ & Number & Percentage & Number & Percentage \\
\hline Never & 1 & $1 \%$ & 3 & $10 \%$ \\
\hline Rarely & 3 & $4 \%$ & 3 & $10 \%$ \\
\hline Sometimes & 12 & $18 \%$ & 4 & $14 \%$ \\
\hline Often & 24 & $36 \%$ & 14 & $48 \%$ \\
\hline Always & 27 & $40 \%$ & 5 & $17 \%$ \\
\hline $\begin{array}{l}\text { Use the Internet as a resource for } \\
\text { nutrition facts at restaurants }\end{array}$ & Number & Percentage & Number & Percentage \\
\hline Never & 3 & $4 \%$ & 3 & $10 \%$ \\
\hline Rarely & 11 & $16 \%$ & 5 & $17 \%$ \\
\hline Sometimes & 13 & $19 \%$ & 9 & $31 \%$ \\
\hline Often & 28 & $42 \%$ & 8 & $28 \%$ \\
\hline Always & 12 & $18 \%$ & 4 & $14 \%$ \\
\hline
\end{tabular}


Figure 6: Solicited Advice: Eat a high protein, low carbohydrate diet

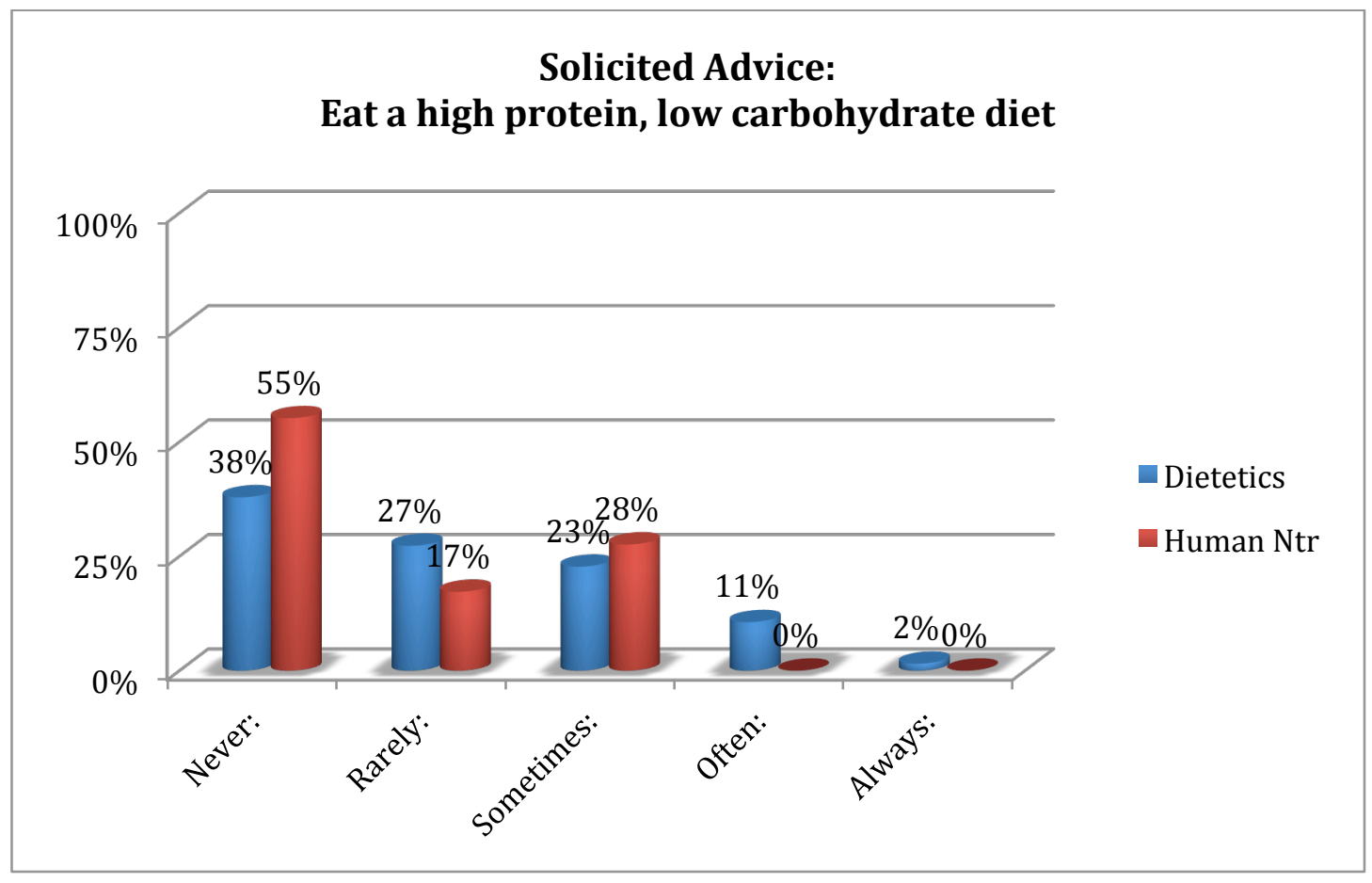

Figure 7: Solicited Advice: Make smart food choices when eating away from home

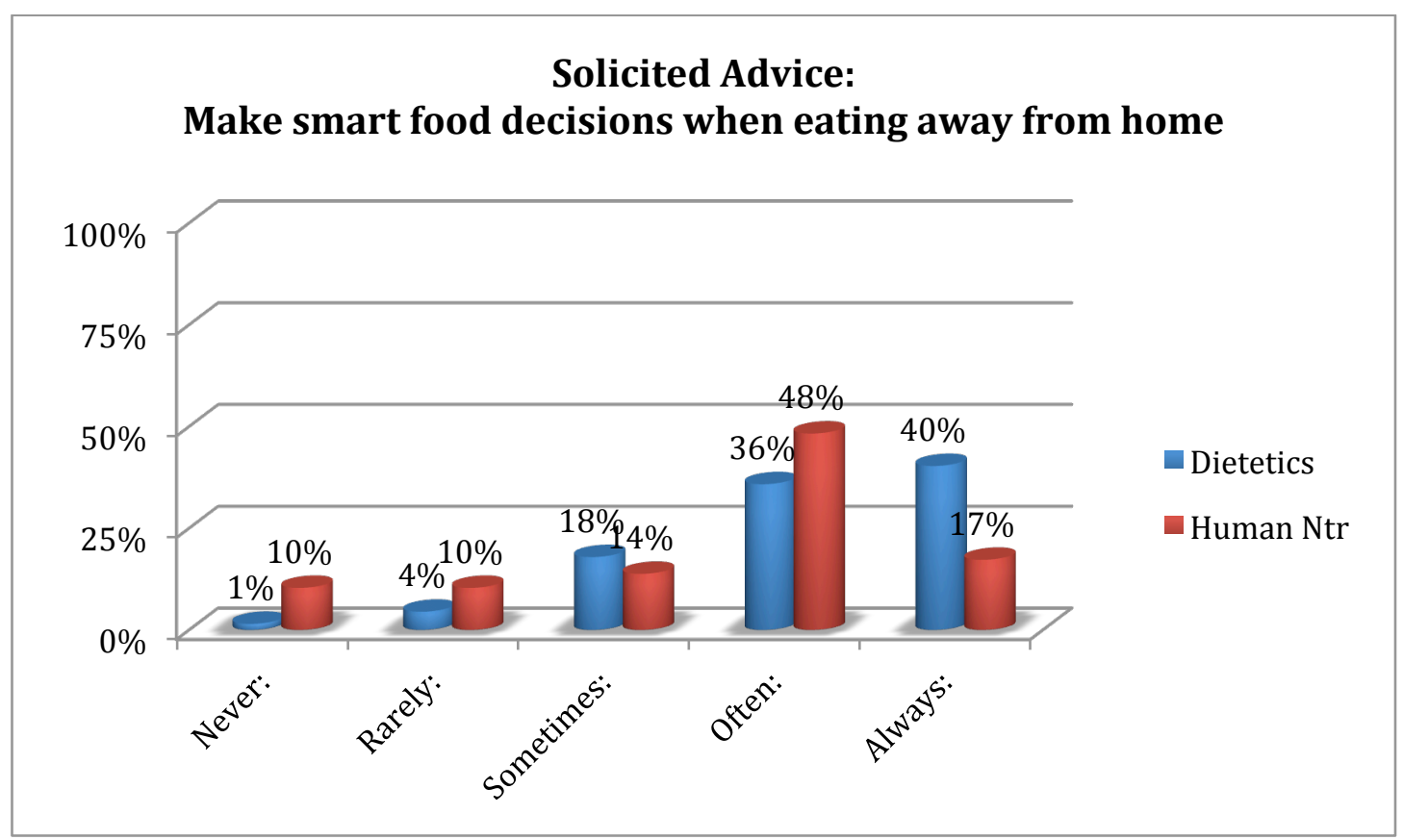


Table 4 (part A): Unsolicited Advice

\begin{tabular}{|c|c|c|c|c|}
\hline \multicolumn{5}{|l|}{ Unsolicited Advice } \\
\hline \multirow{2}{*}{$\begin{array}{l}\text { Question } \\
\text { Eat a high protein, low } \\
\text { carbohydrate diet }\end{array}$} & \multicolumn{2}{|l|}{ Dietetics } & \multicolumn{2}{|c|}{ Human Nutrition } \\
\hline & Number & Percentage & Number & Percentage \\
\hline Never & 37 & $56 \%$ & 16 & $55 \%$ \\
\hline Rarely & 13 & $20 \%$ & 6 & $21 \%$ \\
\hline Sometimes & 11 & $17 \%$ & 6 & $21 \%$ \\
\hline Often & 5 & $8 \%$ & 1 & $3 \%$ \\
\hline Always & 0 & $0 \%$ & 0 & $0 \%$ \\
\hline $\begin{array}{l}\text { Get at least } 60 \text { minutes of } \\
\text { exercise, most days of the week }\end{array}$ & Number & Percentage & Number & Percentage \\
\hline Never & 13 & $19 \%$ & 4 & $14 \%$ \\
\hline Rarely & 7 & $10 \%$ & 3 & $10 \%$ \\
\hline Sometimes & 22 & $33 \%$ & 7 & $24 \%$ \\
\hline Often & 17 & $25 \%$ & 11 & $38 \%$ \\
\hline Always & 8 & $12 \%$ & 4 & $14 \%$ \\
\hline $\begin{array}{l}\text { Eat several smaller meals } \\
\text { throughout the day instead of } 3 \\
\text { large meals }\end{array}$ & Number & Percentage & Number & Percentage \\
\hline Never & 12 & $18 \%$ & 3 & $10 \%$ \\
\hline Rarely & 10 & $15 \%$ & 4 & $14 \%$ \\
\hline Sometimes & 15 & $22 \%$ & 10 & $34 \%$ \\
\hline Often & 25 & $37 \%$ & 6 & $21 \%$ \\
\hline Always & 5 & $7 \%$ & 6 & $21 \%$ \\
\hline $\begin{array}{l}\text { Avoid eating or drinking anything } \\
\text { after dinner }\end{array}$ & Number & Percentage & Number & Percentage \\
\hline Never & 29 & $43 \%$ & 11 & $38 \%$ \\
\hline Rarely & 15 & $22 \%$ & 7 & $24 \%$ \\
\hline Sometimes & 15 & $22 \%$ & 7 & $24 \%$ \\
\hline Often & 5 & $7 \%$ & 4 & $14 \%$ \\
\hline Always & 3 & $4 \%$ & 0 & $0 \%$ \\
\hline $\begin{array}{l}\text { Eat a high protein, high calorie } \\
\text { diet }\end{array}$ & Number & Percentage & Number & Percentage \\
\hline Never & 38 & $57 \%$ & 17 & $59 \%$ \\
\hline Rarely & 24 & $36 \%$ & 6 & $21 \%$ \\
\hline Sometimes & 4 & $6 \%$ & 6 & $21 \%$ \\
\hline Often & 1 & $1 \%$ & 0 & $0 \%$ \\
\hline Always & 0 & $0 \%$ & 0 & $0 \%$ \\
\hline
\end{tabular}


Table 4 (part B): Unsolicited Advice

\begin{tabular}{|c|c|c|c|c|}
\hline \multicolumn{5}{|l|}{ Unsolicited Advice } \\
\hline \multirow{2}{*}{$\begin{array}{l}\text { Question } \\
\text { Avoid calorically dense foods and } \\
\text { beverages }\end{array}$} & \multicolumn{2}{|l|}{ Dietetics } & \multicolumn{2}{|c|}{ Human Nutrition } \\
\hline & Number & Percentage & Number & Percentage \\
\hline Never & 15 & $22 \%$ & 4 & $14 \%$ \\
\hline Rarely & 8 & $12 \%$ & 4 & $14 \%$ \\
\hline Sometimes & 17 & $25 \%$ & 10 & $34 \%$ \\
\hline Often & 21 & $31 \%$ & 9 & $31 \%$ \\
\hline Always & 6 & $9 \%$ & 2 & $7 \%$ \\
\hline $\begin{array}{l}\text { Consume fewer alcoholic } \\
\text { beverages }\end{array}$ & Number & Percentage & Number & Percentage \\
\hline Never & 15 & $23 \%$ & 8 & $28 \%$ \\
\hline Rarely & 13 & $20 \%$ & 4 & $14 \%$ \\
\hline Sometimes & 19 & $29 \%$ & 10 & $34 \%$ \\
\hline Often & 15 & $23 \%$ & 6 & $21 \%$ \\
\hline Always & 4 & $6 \%$ & 1 & $3 \%$ \\
\hline $\begin{array}{l}\text { Make smart food decisions when } \\
\text { eating away from home }\end{array}$ & Number & Percentage & Number & Percentage \\
\hline Never & 14 & $21 \%$ & 4 & $14 \%$ \\
\hline Rarely & 2 & $3 \%$ & 4 & $14 \%$ \\
\hline Sometimes & 18 & $27 \%$ & 7 & $24 \%$ \\
\hline Often & 17 & $25 \%$ & 8 & $28 \%$ \\
\hline Always & 16 & $24 \%$ & 6 & $21 \%$ \\
\hline $\begin{array}{l}\text { Use the Internet as a resource for } \\
\text { nutrition facts at restaurants }\end{array}$ & Number & Percentage & Number & Percentage \\
\hline Never & 16 & $25 \%$ & 5 & $17 \%$ \\
\hline Rarely & 12 & $18 \%$ & 8 & $28 \%$ \\
\hline Sometimes & 17 & $26 \%$ & 6 & $21 \%$ \\
\hline Often & 11 & $17 \%$ & 6 & $21 \%$ \\
\hline Always & 9 & $14 \%$ & 4 & $14 \%$ \\
\hline
\end{tabular}


Figure 8: Unsolicited Advice: Eat a high protein, low carbohydrate diet

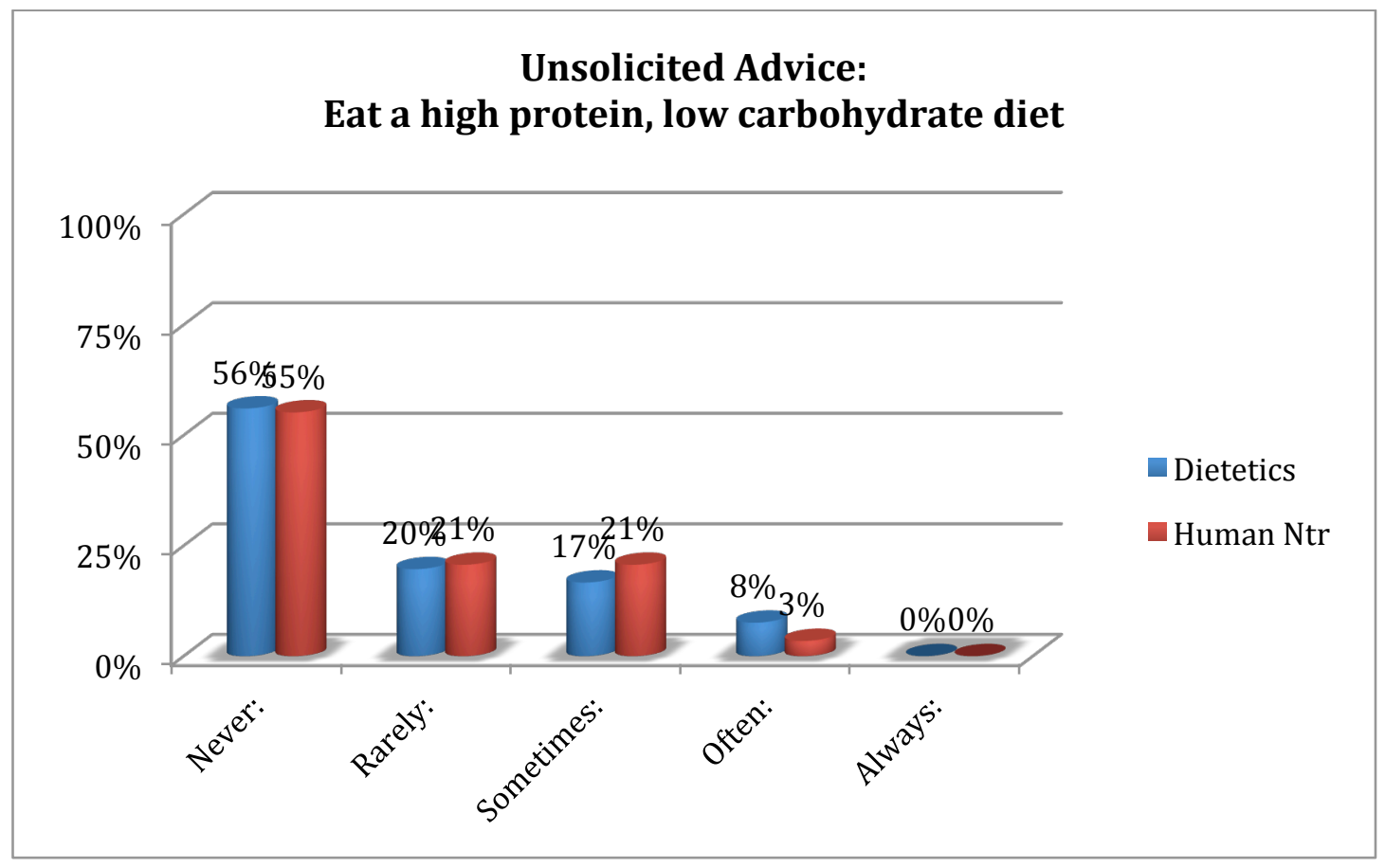

Figure 9: Unsolicited Advice: Make smart food choices when eating away from home

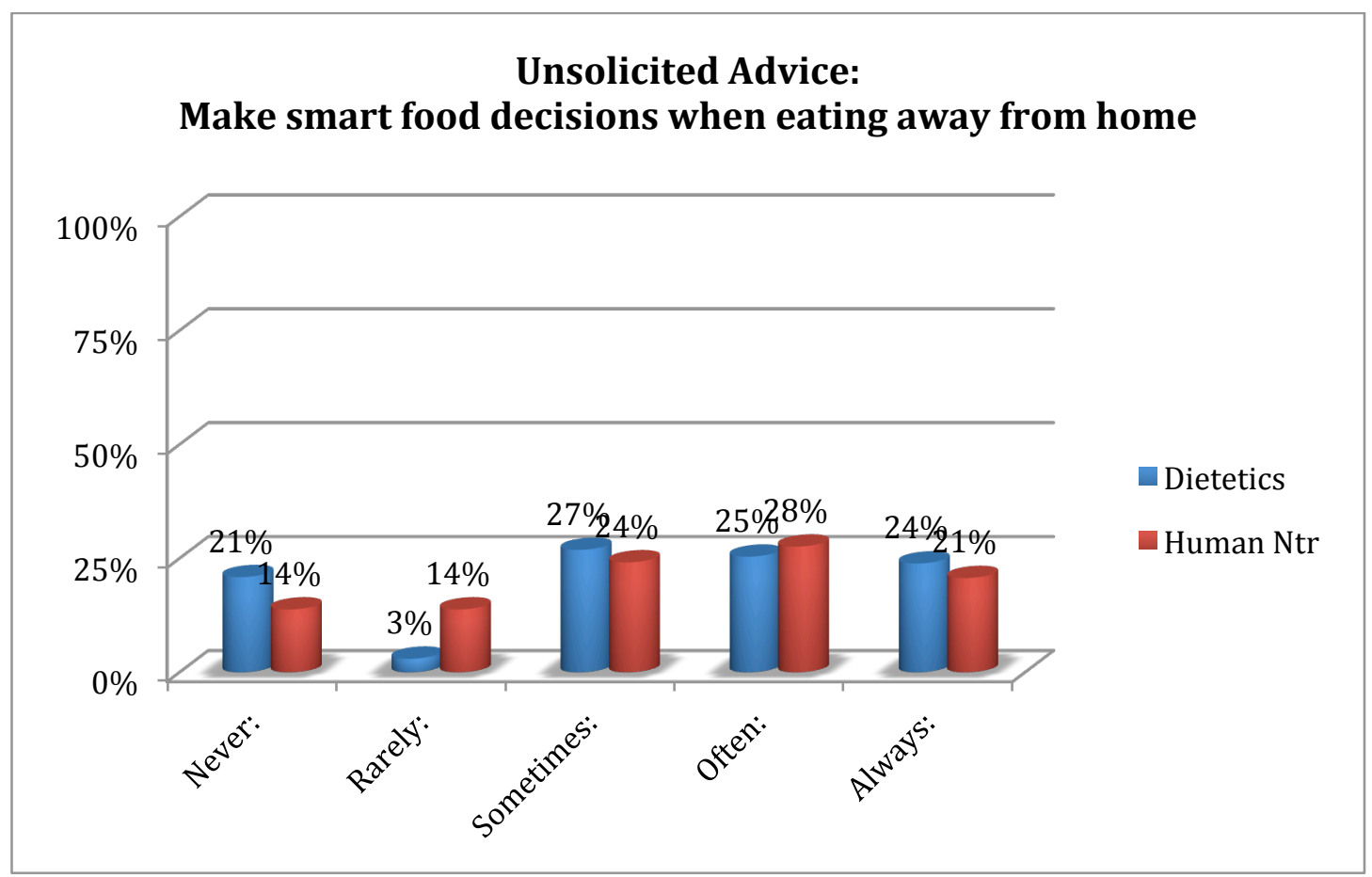


Figure 10: Stressor: Relationship Ends

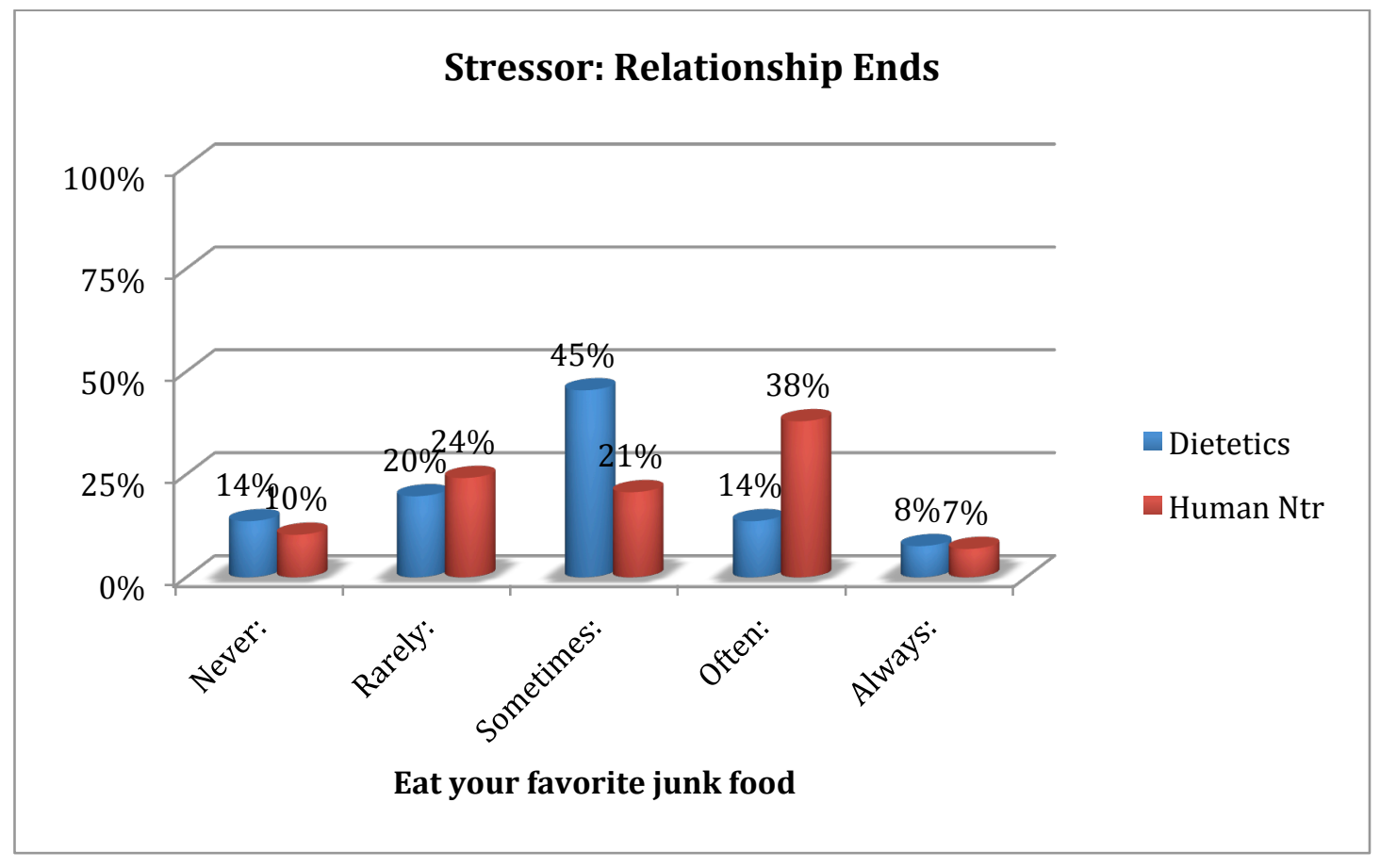




\section{Chapter 5: Discussion}

\section{Scope of Dietetics Practice Implications}

The Scope of Dietetics Practice and the Code of Ethics for the Dietetics Profession were created by the American Dietetic Association to define the functions and duties of people who consider themselves dietetic professionals. Within the confines of the Scope of Dietetics Practice, anyone who does not have state licensure, credentials from the Commission on Dietetic Registration, specialty certificates, advanced practice certification, or an advanced degree is not considered a dietetics professional, and therefore, should not act as one. Included in this group are undergraduate students studying and preparing to become nutrition professionals. While they learn the most up-to-date information possible and possess a great deal of knowledge, students have not yet become credentialed dietetics professionals and should not assume the roles of the credentialed professional by giving advice or performing any other duties given only to those people who have earned their credentials.

The five questions in this study regarding the Scope of Dietetics Practice were meant to evaluate the participants' knowledge and comprehension of the Scope of Dietetics Practice, as well as examine their implementation of it.

Overall, the participants who indicated a better understanding of the Scope of Dietetics Practice and the Dietetics Code of Ethics and those willing to refer family members and friends to people outside of their scope of practice tended to give more appropriate advice in given situations and when asked. Those who expressed an increased tendency to diagnose others gave more unsolicited advice, and their 
advice was not generally advisable. In the comparison of participants in a didactic program in dietetics (DPD) to those studying human nutrition, the DPD participants were more likely to model positive, healthy food-related behaviors, advise others to be wary of nutrition content when eating out and where to find that information, and understood more fully how to advise a person who was underweight and wanted to healthfully gain weight. The results of this study indicate a need to address and teach the Scope of Dietetics Practice and Dietetics Code of Ethics to students studying nutrition, regardless of their future career aspirations.

\section{Discussion}

The purpose of this study was to investigate whether nutrition students use their knowledge to deal with stressful situations, work within the Scope of Dietetics Practice, and model healthy food-related behaviors. The research questions aimed to discover if nutrition students practice what they learned in class, if they give advice to others within the Scope of Dietetics Practice, if they utilize food-related behaviors to cope with stressful situations, and if there are any significant differences between dietetics students and those studying human nutrition.

To answer the first question, "do undergraduate nutrition students practice what they learn in class," responses to the questions regarding Scope of Dietetics Practice were analyzed for the sample; the sample was also tested for significance between majors. Incorporated with answering the first question regarding Scope of Dietetics Practice were the other three questions: differences in solicited and unsolicited advice to family members and individuals, and stressful situations; all 
categories were evaluated for differences between dietetics participants and human nutrition students.

1. "I only deliver information allowed at the student level by the CDR, as specified in the Scope of Dietetics of Practice."

Answering this question, most of the participants (51\%) responded "neutral;" this indicates that they neither agreed nor disagreed with the statement, and can be interpreted that most participants know that sometimes they deliver more information than permitted by the Commission on Dietetic Registration, or they do not know what they can and cannot tell others as a student.

Participants who were more likely to exclusively deliver information allowed at the student level by the Commission on Dietetics Registration (CDR), more likely to advise others with widely recognized practices, such as eating small meals throughout the day and avoiding calorically dense foods and beverages to lose weight. The participants who were more likely to only give information permitted by CDR at the student level were less likely to advise or participate in activities that may be considered "extreme" or inappropriate for certain populations, such as advising the general public to exercise for sixty minutes per day, most days.

\section{2. "I comprehend the scope of practice."}

The responses to this question were split mainly between "neutral" (33\%) and "agree" (46\%); this indicates that the majority of participants felt that they somewhat understood the Scope of Dietetics Practice. However, in that so many 
participants responded "neutral," curriculum developers may want to consider increasing the amount of time spent on the Scope of Dietetics Practice in a professional ethics course.

Participants who were more likely to have a better comprehension of the Scope of Dietetics Practice tended to give solicited and unsolicited recommendations for exercise in a variety of scenarios, recommend that others read labels in the groceries and at restaurants, and they were more likely to believe that they give good advice and that their BMI support their credibility. The participants who were more likely to comprehend the scope of practice were less likely to recommend calorically dense foods and beverages to any population and they tended not to cope with stressful situations by consuming junk foods.

\section{3. "I refer friends and family to other sources that were outside my scope of practice."}

The majority of the participants $(76 \%)$ indicated that they refer friends and family to sources for information when the request is beyond the participants' abilities within the scope of practice by selecting "agree" or "strongly agree." This shows that most participants understand the confines of the Scope of Dietetics Practice and they know when they should tell others to consult someone else, such as a dietitian or a certified diabetes educator.

Participants who were more likely to refer their friends and family to other sources were more likely to give generally recognized advice, such as avoiding calorically dense foods and beverages for a person trying to lose weight, eating frequently throughout the day for a person trying to gain a healthy amount of 
weight, and tended to use positive mechanisms to manage stress, such as calling a friend or family member and exercising. However, these participants were also more likely to consume alcoholic beverages to deal with unruly neighbors or roommates. Finally, participants who were more likely to comprehend the scope of practice were more likely to model and teach others with credible, reliable information. Participants who were more likely to comprehend the Scope of Dietetics Practice were less likely to give poor or inappropriate advice, such as unsolicited diet advice and incorrect advice for a given scenario.

\section{4. "I abide by the Dietetics Code of Ethics."}

Seventy-four percent of the participants indicated that they "agree" or "strongly agree" that they obey the Dietetics Code of Ethics, as compared to the $60 \%$ of participants who indicated that they "agree" or "strongly agree" that they comprehend the Scope of Dietetics Practice. This indicates that a strong majority of all upper level nutrition students believe that they follow the principles outlined in the code of ethics, but only $60 \%$ believe that they comprehend the Scope of Dietetics Practice adequately; this is interesting since the Code of Ethics is a part of the Scope of Dietetics Practice.

Participants who were more likely to follow the Dietetics Code of Ethics were more likely to give a variety of generally accepted advice, both solicited and unsolicited. This advice included exercising sixty minutes most days of the week, using the Internet as a resource for nutrition information, recommending abstention from food and beverages after dinner, and consuming fewer alcoholic beverages. 
These participants also tended to recommend an unsolicited diet of high protein, high calorie; this may be because participants interpreted the diet as a way to healthfully gain weight and muscle, especially with increased protein. While consuming increased amounts of protein does not lead to increased muscle mass, many college students believe that it does; this is a fallacy that should be addressed throughout nutrition courses. Finally, participants who tended to abide the Dietetics Code of Ethics better tended to use junk food as a way to deal with some stressful situations, but used exercise and schoolwork in other instances.

Participants who were more likely to abide by the Dietetics Code of Ethics were less likely to recommend specific solutions to specific situations. This may be because the participants knew that, as students, they are ethically bound not to give specific nutritional advice to anyone. Another possibility is that the dietetics participants knew that research occurs on a regular basis that can support or disprove such dogmatic ideas about nutrition. Finally, these participants were less likely to use schoolwork as a stress relief in situations regarding relationship issues and unexpected expenses; they also tended not to consume junk food as a way to handle school-related stress.

5. "I use nutrition information to diagnose health conditions in family members and individuals."

Responses to this question were split between "disagree" (24\%), "neutral" (30\%), and "agree" (27\%); this is interesting because, according to the Scope of Dietetics Practice, non-credentialed individuals, such as college students, should not 
use their knowledge to diagnose health conditions in anyone. The trend showed that dietetics participants were less likely to respond positively to this statement than human nutrition participants, but the trend was not statistically significant.

Participants who were more likely to use nutrition information to diagnose health conditions in family members and individuals were more likely to recommend extreme high protein, low carbohydrate and high protein, high calorie diets in both solicited and unsolicited settings. Overall, this group of individuals gave the highest amount of statistically significant unsolicited advice. Other areas of significant unsolicited advice included eating small meals throughout the day, not consuming anything after dinner, avoiding calorically dense foods and beverages, and consuming fewer alcoholic beverages. In addition, the participants more likely to use nutrition information to diagnose others were more likely to make specific recommendations in the given scenarios, some of which were not based on research or evidence based data. Finally, these participants were more likely to use junk food as a means of coping with a variety of stressful situations, though they also reported an increased tendency of following the dietary guidelines on alcohol consumption.

While this group of participants were more likely to give a variety of unsolicited advice, as described previously, they were less likely to make specific recommendations to overweight/obese individuals planning to lose weight or avoid weight gain. 


\section{Course of Study Implications}

Evaluation of differences between participants in a didactic program in dietetics and those in a pre-professional nutrition program was one of the key purposes of this study. The results showed that participants in DPDs - the future dietitians - were more likely to recommend making healthier food choices when eating away from home and using the Internet as a resource for restaurant nutrition information, as well as to give positive and healthy advice to an underweight individual who wants to gain weight appropriately. Additionally, participants in DPDs were significantly more likely to believe that their BMI and eating and exercise behaviors supported their credibility as future nutrition professionals.

Conversely, pre-professional participants with human nutrition as their undergraduate course of study were more likely to believe that they modeled correct portion sizes when eating with family and friends. Because the average BMI of human nutrition participants was higher (24.3) than the average dietetics participants (21.9), this trend may be explained through dietetics students learning more about proper portion size and know they do not model it around others, while human nutrition students do not have this same knowledge base. Another plausible explanation for the differences in BMI and portion control implementation is that dietetics students tended to practice portion control more often than human nutrition students. Participants majoring in human nutrition were more likely to use schoolwork to cope with the stress of a family member and junk food to cope with the stress of the end of a relationship. By analyzing each of the survey questions 
compared to the Scope of Dietetics Practice questions and major, all of the research questions were addressed.

Bias, Limitations, and Future Research

The data collection method used in the study was an online survey given to college students; because they were recruited through registration in an upper-level nutrition class, the participants may have answer in ways that were "expected," instead of with the response that was the most accurate for them. One of the primary areas expected to include bias was alcohol consumption.

This study served as a useful first look at students studying nutrition and their practice and application of nutrition knowledge. Limitations of the study include complicated questions regarding referral of family and friends to sources outside the participants' scope of practice; participants may have inferred that this was an official referral, not a general recommendation to consult a credentialed professional. In addition, participants may have interpreted the scenario involving an underweight individual as a male wanting to "bulk up," not as a person with a detrimentally low BMI as was intended. Many of the participants responded in ways that discouraged the use of the Internet as a reliable resource; they may be wary of excessive Internet use and consulting an online source instead of the information posted in the restaurant.

Areas for future research include expansion of the sample to increase the generalizeability, exploration of attitudes toward ways to increase muscle mass, and methods necessary to gain weight in a healthy manner. In addition, further 
research should be done to explore the differences between dietetics students and students studying human nutrition.

\section{Final Conclusions}

College students face the recurring challenge of weight management. For most students, this period serves as the first time they are explicitly responsible for their food selections and exercise habits. This challenge applies to all college students, including those studying nutrition. Some students study nutrition as a means to earn a bachelor's degree and then attend a professional school, such as medical school, pharmacy school, and dental school, as well as any other health-based profession. However, other students study nutrition to become registered dietitians, working their way through a didactic program in dietetics.

Regardless of their intended profession, most students who study nutrition during their undergraduate career freely give advice, are asked for it, and constantly encounter situations where they model health behaviors to their family members and friends.

Society plays an important role in health behaviors, reaching to areas such as body image, exercise expectations, and food-related behaviors. This study demonstrated this through the results of the stress and behavior section. One of the findings was that human nutrition students were significantly more likely to consume junk food to manage the stress of a relationship ending. Societal cues likely play a part in this trend - on a wide variety of television shows and movies, if a character encounters this particular scenario, they often reach for a pint of ice 
cream, a bag of potato chips, or another type of junk food. Because society plays such an integral role in how individuals act and what they believe.

It is vital for all students studying nutrition to know and understand the Scope of Dietetics Practice, as well as the Dietetics Code of Ethics so that they may accurately and appropriately answer questions. One of the key features of the Scope of Dietetics Practice is that it applies to all people who give nutrition advice, regardless of their membership in the American Dietetic Association, status of credentials, and level of education. Because of this, the American Dietetic Association holds all people responsible to a standard level of ethics and professionalism should they dispense any sort of nutrition recommendations. By correctly advising others in the capacity allowed through the Scope of Dietetics Practice and always modeling positive health behaviors, students will serve as an excellent example to their communities and will continue to live by example in their future profession. 


\section{Appendix A: Survey}

\section{Practice and Application of Knowledge by Nutrition Students}

\section{Consent Waiver}

\section{Dear Nutrition Major:}

You have been chosen to participate in a research study that examines the nutritionrelated behaviors of nutrition students. The study is limited to students at the University of Kentucky and the work is in part to fulfill the requirements of the Master's Degree program in Hospitality and Dietetics Administration.

Although you will not get personal benefit from taking part in this research study, your responses may help us understand more about nutrition-related behaviors in college students who are studying nutrition.

We hope to receive completed questionnaires from about 250 people, so your answers are important to us. Of course, you have a choice about whether or not to complete the survey/questionnaire, but if you do participate, you are free to discontinue at any time.

The survey/questionnaire will take about 25 minutes to complete.

You will not be paid for taking part in this study, but if you chose to submit your e-mail address, you will be entered into a drawing for a $\$ 50 \mathrm{gift}$ card.

There are no known risks to participating in this study.

Your response to the survey is anonymous which means no names will appear or be used on research documents, or be used in presentations or publications. The research team will not know that any information you provided came from you, nor even whether you participated in the study.

If you have questions about the study, please feel free to ask; my contact information is given below. If you have complaints, suggestions, or questions about your rights as a research volunteer, contact the staff in the University of Kentucky Office of Research Integrity at 859-257-9428 or toll-free at 1-866-400-9428.

Thank you in advance for your assistance with this important project.

Sincerely, 


\section{Practice and Application of Knowledge by Nutrition Students}

Jennifer Boyee

Nutrition and Food Sciences, University of Kentucky

E-MAIL: jennifer.boyee@uky.edu

Advisor: Dr. Hazel Forsythe

E-MAIL: nfshazel@email.uky.edu

PHONE: 859-257-4146

Agree 


\section{Practice and Application of Knowledge by Nutrition Students}

2. Demographics

These questions concern demographic and will be used only to establish trends within this study.

1. Gender

Female

Male

2. Height

Feet

Inches

3. Weight

Pounds

4. Age
$18 \cdot 20$
$21 \cdot 23$
$24 \cdot 30$
( $31+$

5. Please choose the ethnicity with which you most closely identify.
African American
Asian/Pacific Islander
Biracial
Caucasian
Hispanic
Native American
Middle Eastern
Other (plaase specify) 
Practice and Application of Knowledge by Nutrition Students

6. Current academic standing

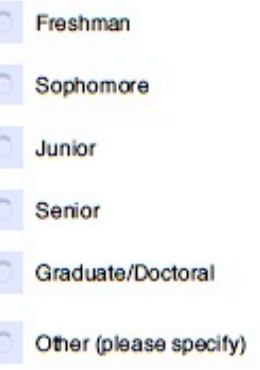

7. Current major

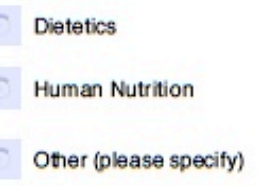

8. Pick the style of eating that best resembles your typical dietary preferences. Please check one (1) of the following.

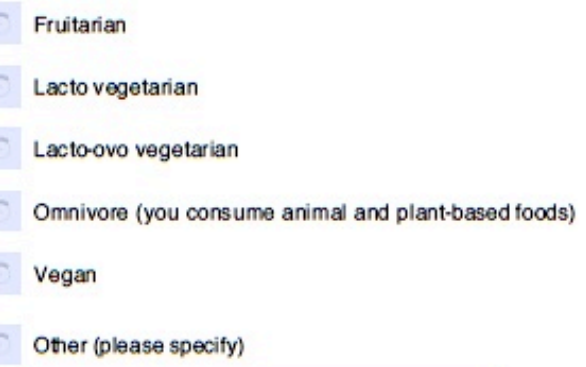

9. Please indicate the dollar amount that most closely represents your total family income.

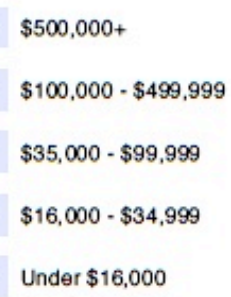




\section{Practice and Application of Knowledge by Nutrition Students}

10. Who or what is the primary source paying your college tuition?
FAFSA student loans
Parents
Savings
Scholarships
(C) Working
Other (please specify) 


\section{Practice and Application of Knowledge by Nutrition Students}

3. Questionnaire

Please select the best answer for each question.

1. What SOLICITED nutrition-related advice do you give to others?

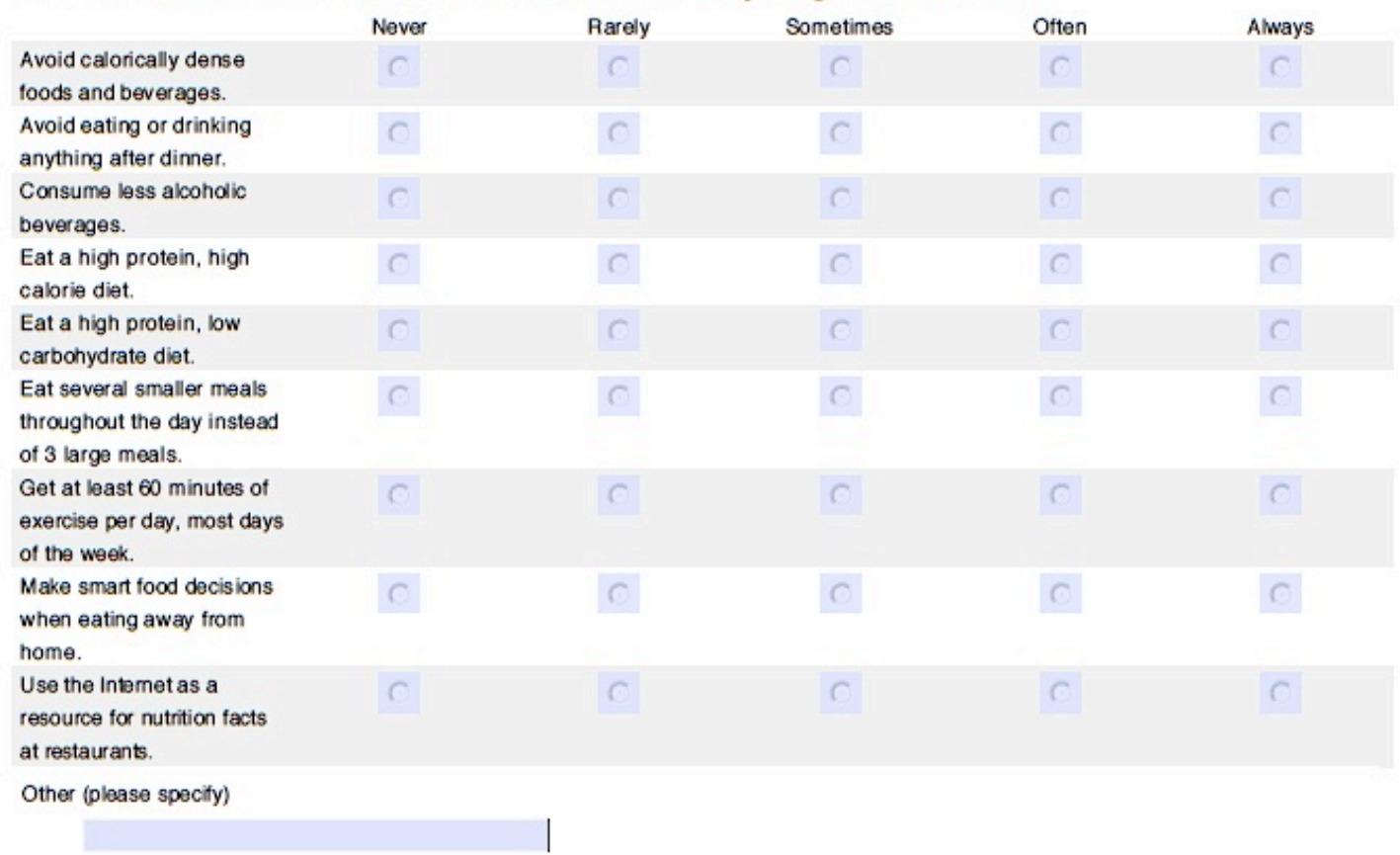

2. Who typically receives your SOLICITED advice?

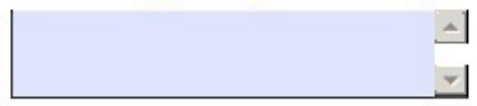


Practice and Application of Knowledge by Nutrition Students

3. What UNSOLICITED nutrition-related advice do you give to others?

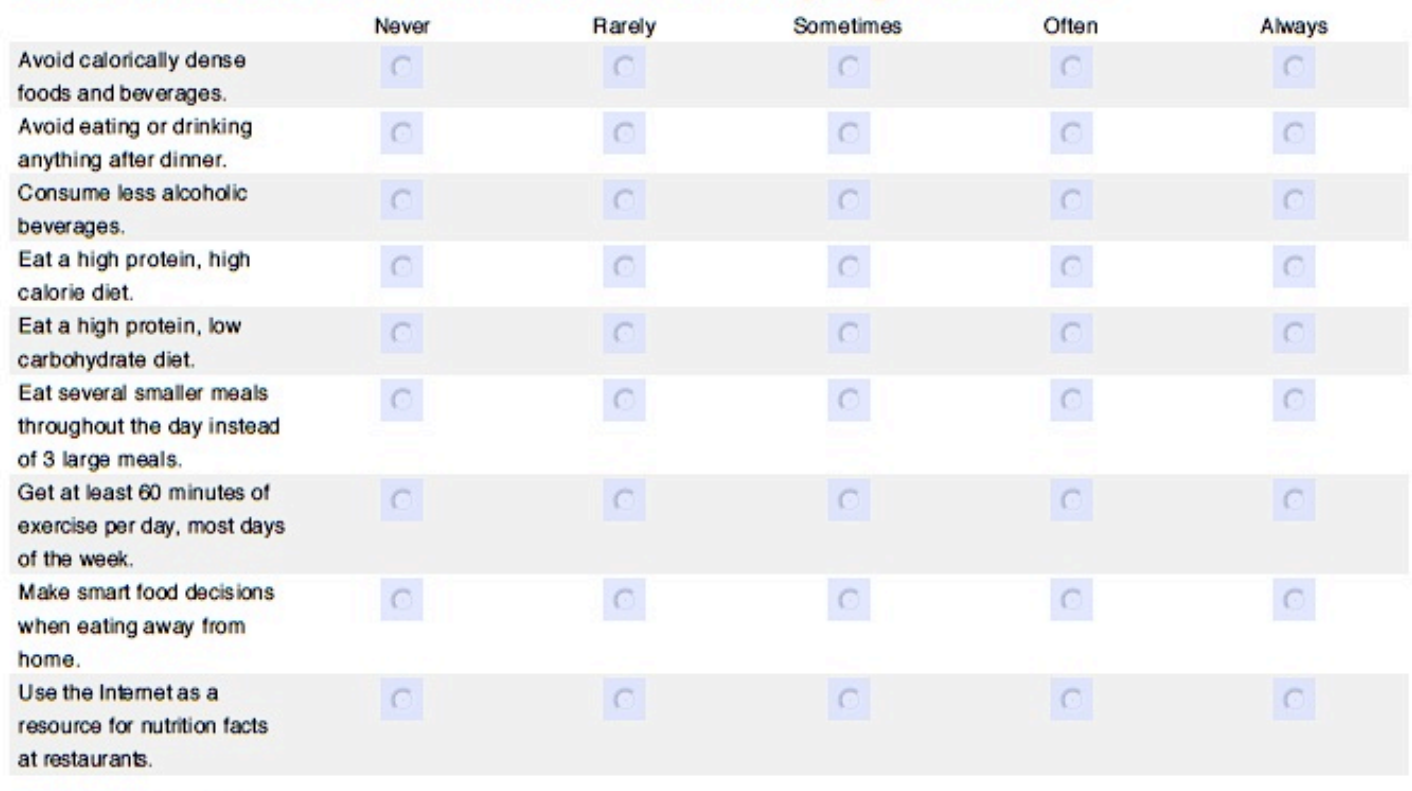

Other (please specify)

4. Who typically receives your UNSOLICITED advice?

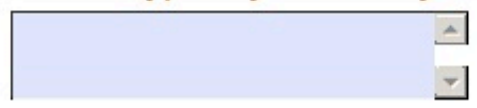


Practice and Application of Knowledge by Nutrition Students

5. A friend or family member is overweight or obese and has been inspired to attain a healthy weight. Because they know that you are in the nutrition field, they come to you for advice on how to meet their weight goals.

What do you tell them?

\begin{tabular}{|c|c|c|c|c|c|}
\hline & Never & Rarely & Sometimes & Often & Always \\
\hline $\begin{array}{l}\text { Avoid calorically dense } \\
\text { foods and beverages. }\end{array}$ & C & $r$ & C & C & r \\
\hline $\begin{array}{l}\text { Avoid eating or drinking } \\
\text { anything after dinner. }\end{array}$ & r & $r$ & C & C & C \\
\hline $\begin{array}{l}\text { Consume less alcoholic } \\
\text { beverages. }\end{array}$ & $r$ & C & C & C & r \\
\hline $\begin{array}{l}\text { Eat a high protein, high } \\
\text { calorie diet. }\end{array}$ & C & C & r & C & r \\
\hline $\begin{array}{l}\text { Eat a high protein, low } \\
\text { carbohydrate diet. }\end{array}$ & r & r & C & C & r \\
\hline $\begin{array}{l}\text { Eat several smaller meals } \\
\text { throughout the day instead } \\
\text { of } 3 \text { large meals. }\end{array}$ & C & $\mathrm{C}$ & C & r & C \\
\hline $\begin{array}{l}\text { Get at least } 60 \text { minutes of } \\
\text { exercise per day, most days } \\
\text { of the week. }\end{array}$ & $r$ & C & C & C & C \\
\hline $\begin{array}{l}\text { Make smart food decisions } \\
\text { when eating away from } \\
\text { home. }\end{array}$ & C & C & C & C & C \\
\hline $\begin{array}{l}\text { Use the Intemet as a } \\
\text { resource for nutrition facts } \\
\text { at restaurants. }\end{array}$ & C & C & C & C & r \\
\hline Other (please specify) & & & & & \\
\hline
\end{tabular}


Practice and Application of Knowledge by Nutrition Students

6. A friend or family member is a freshman in college this year and they have heard that everyone gains $10-20$ pounds during their first year in college. Because they know that you are in the nutrition field, they come to you for advice on how to maintain their current weight.

What do you tell them?

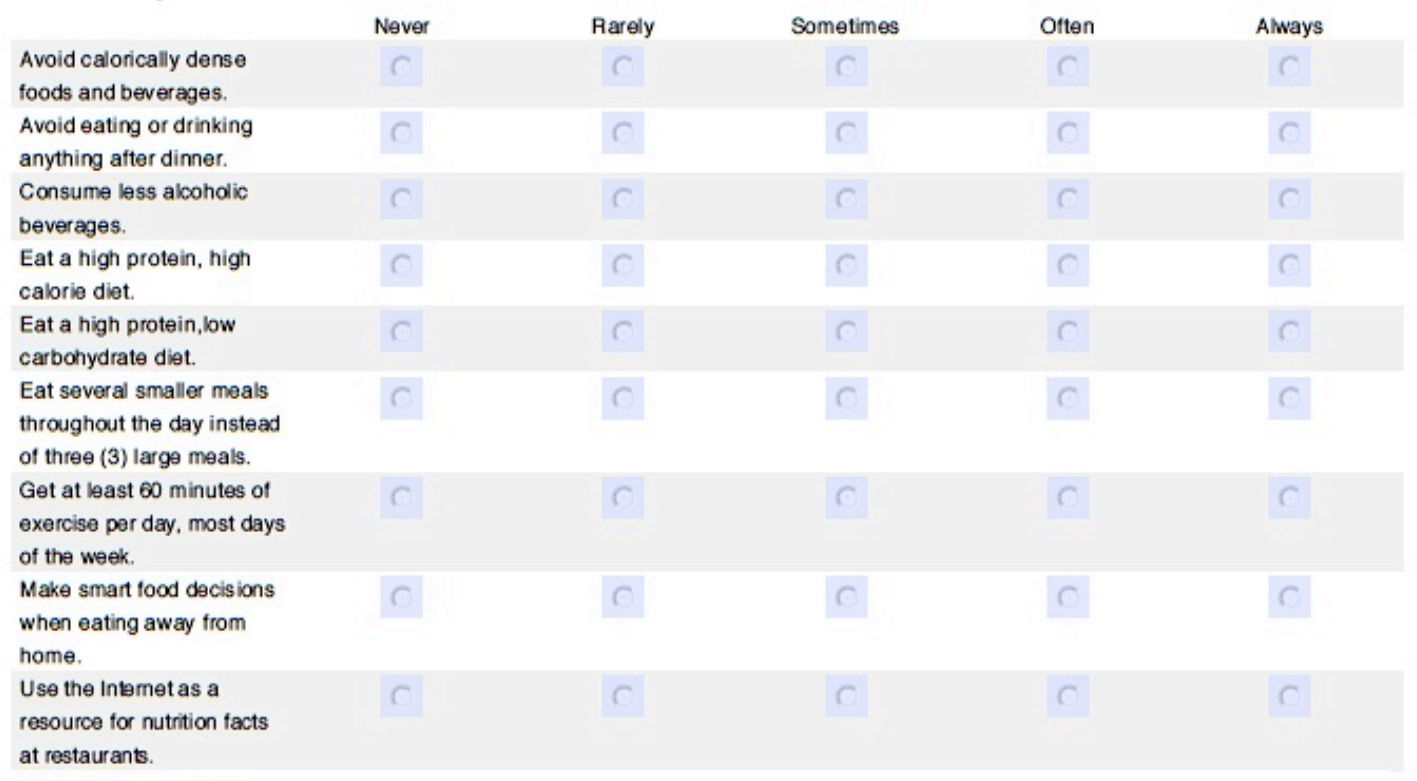

Other (please specify) 
Practice and Application of Knowledge by Nutrition Students

7. A friend or family member is underweight and they want to achieve a healthy weight. Because they know that you are in the nutrition field, they come to you for advice on how to healthfully gain weight.

What do you tell them?

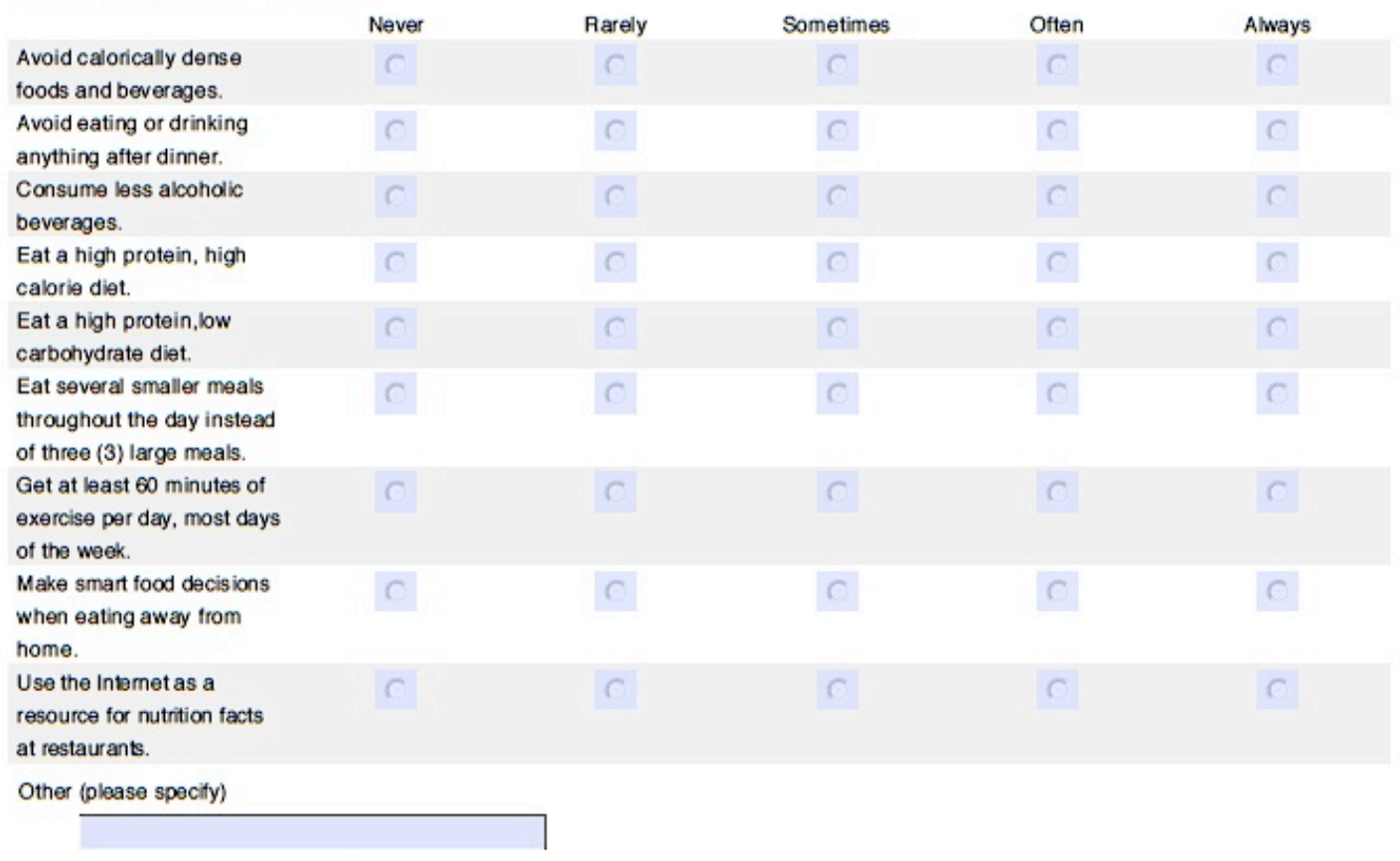

8. You and your significant other break up with each other. How do you cope with the stress?

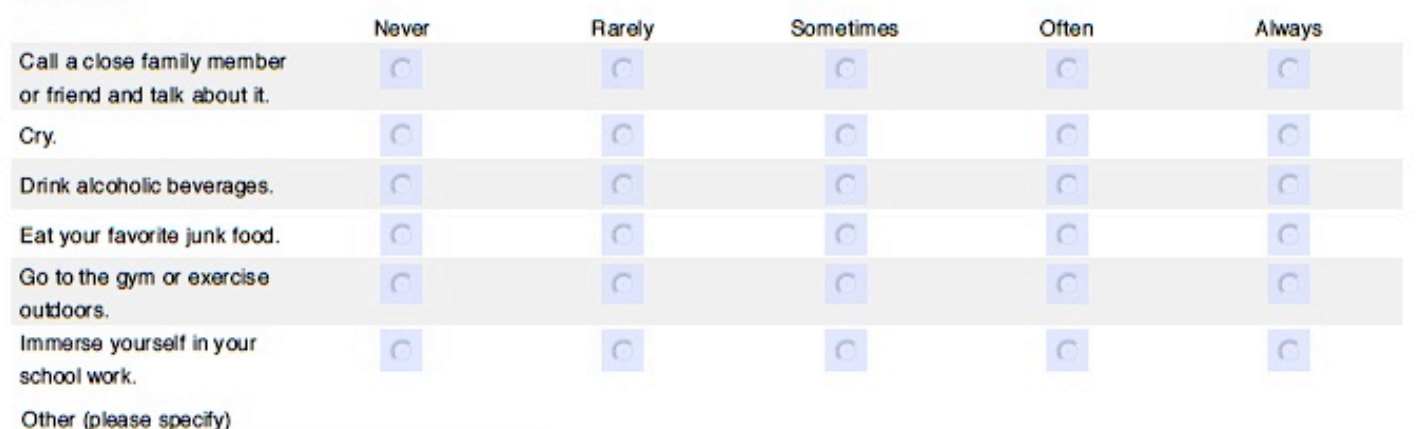

Other (please specify) 


\section{Practice and Application of Knowledge by Nutrition Students}

9. You encounter an unexpected expense (your car needs pricey repairs, you have an emergency medical procedure, etc.). How do you cope with the stress?

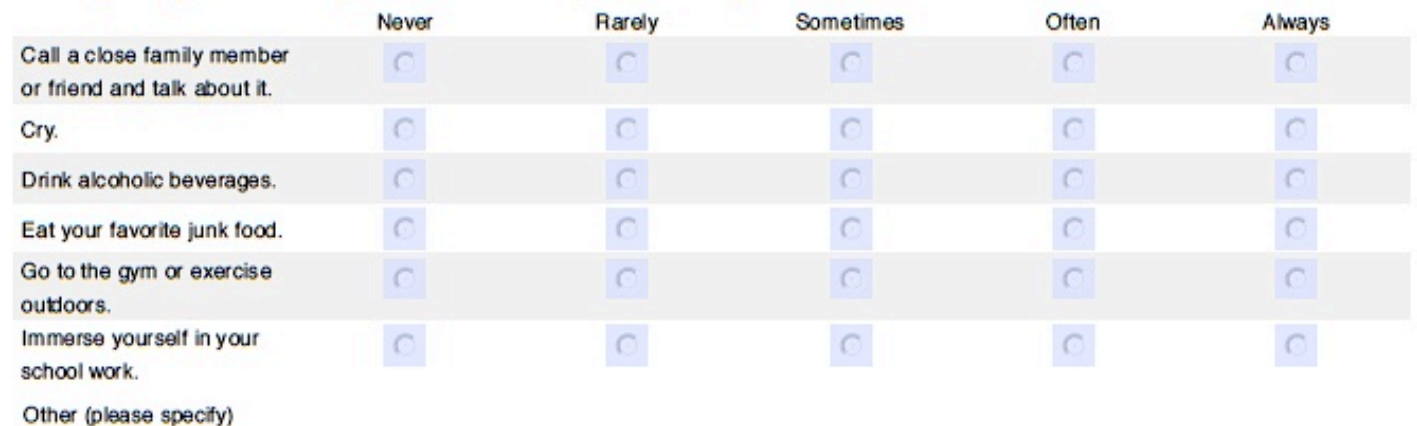

Other (plaase specify)

10. You experience the death of a close family member or friend. How do you cope with the stress?

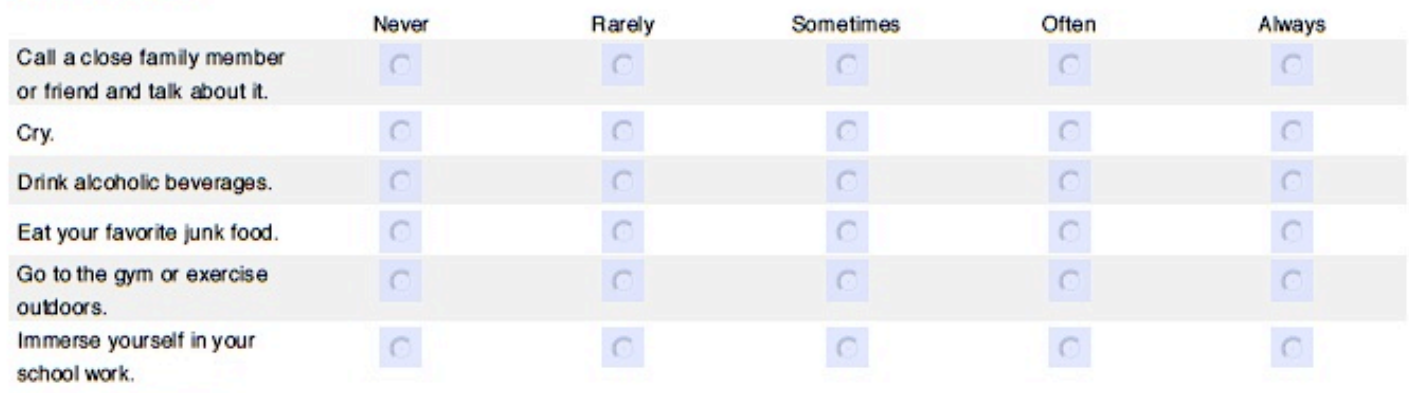

Other (please specify)

11. You must achieve and maintain a certain GPA (for a scholarship membership in a club or sports team, retain parental financial support, etc.). How do you cope with the stress?

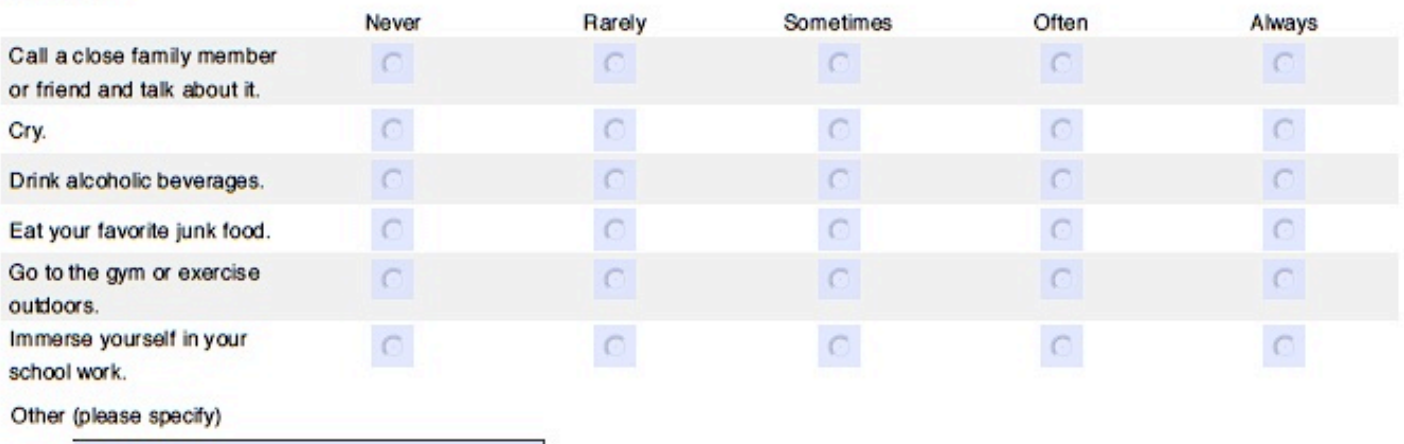




\section{Practice and Application of Knowledge by Nutrition Students}

12. You have extremely irritating neighbors or roommates - they party frequently and loudly, at inappropriate times of day, and throughout midterms and finals weeks. How do you cope with the stress?

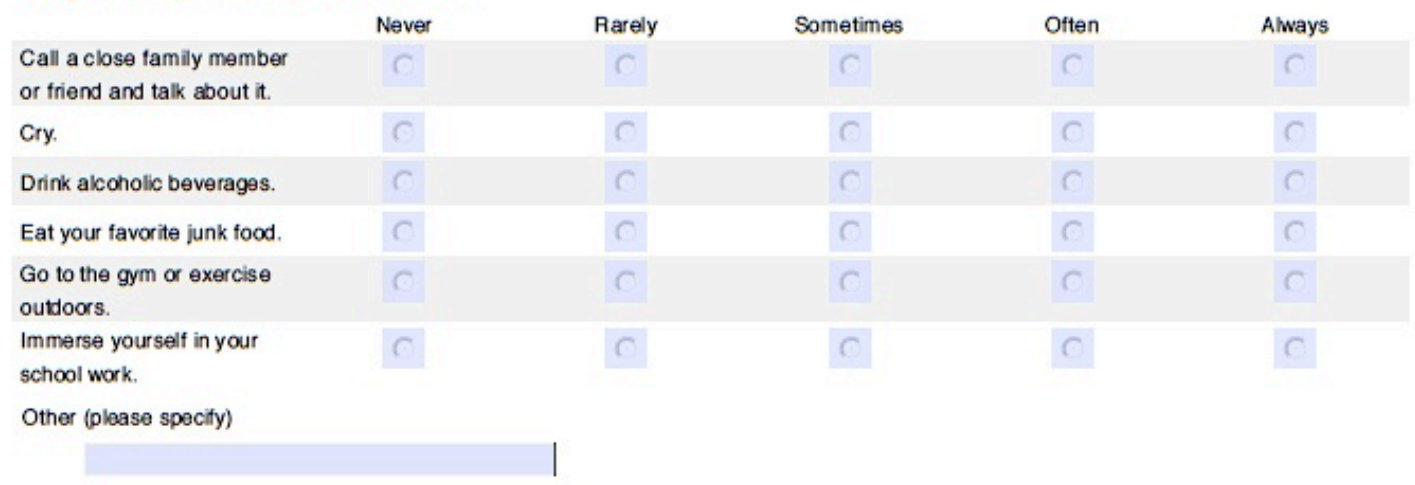

13. Your midterms and finals weeks are packed with several difficult exams. How do you cope with the stress?

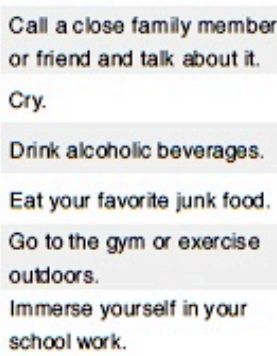




\section{Practice and Application of Knowledge by Nutrition Students}

14. Scope of Practice: Please rate the the level that corresponds best to you.

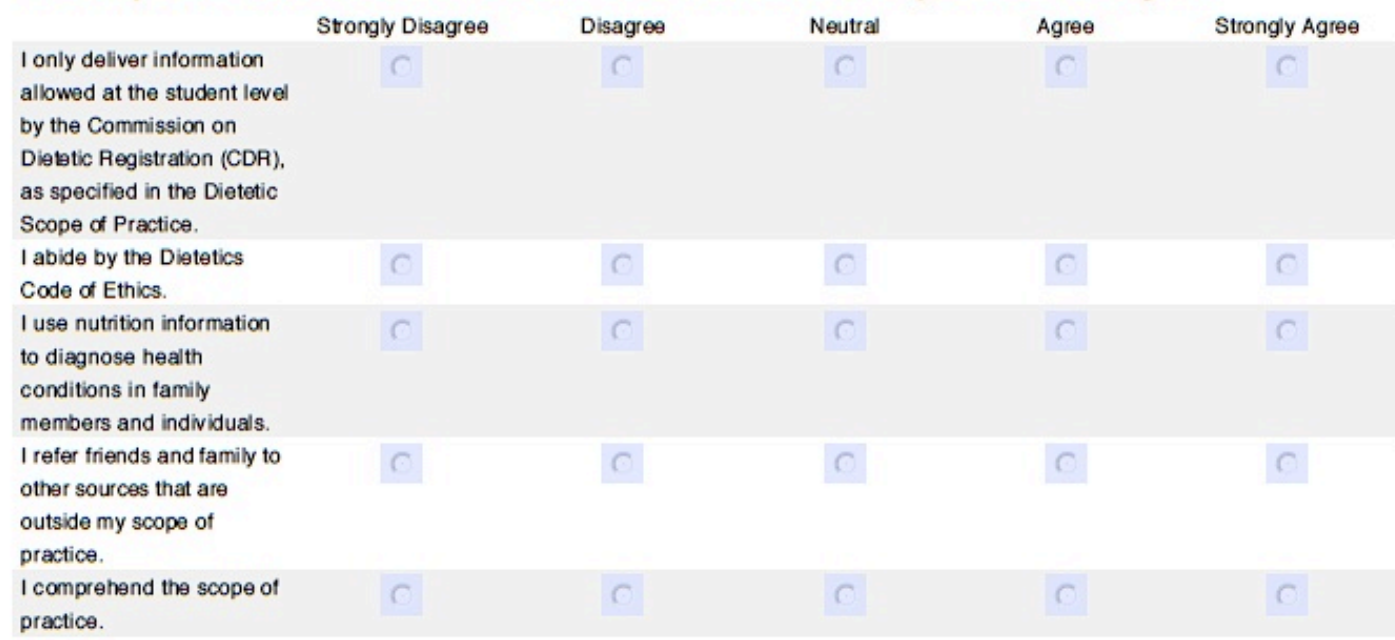

15. Modeling Food and Nutrition Behaviors: Please rate the level that corresponds best to you.

I use information from the
dietary guidelines to model
nutrition behaviors.
I advise friends and family
to use the dietary
guidelines to make food
and nutrition decisions.
When grocery shopping
with friends and family, I
encourage them to read
the nutrition facts labels.
I use correct portion sizes
when eating with friends
and family.
I follow dietary guidelines
in my alcohol consumption
(no more than 1 standard
drink per day for women; no
more than 2 standard drinks
per day for men).




\section{Practice and Application of Knowledge by Nutrition Students}

16. Credibility and Reliability Behavior: Please rate the level that best corresponds to you.

My health behaviors (eating and exercise)

support my credibility with family and individuals.

The information I give to others is usable, practical,

and accurate.

1 incorporate low fat, high

fiber foods into my diet on

a regular basis.

Individuals and family

members believe my

information is credible

based on my years in

school.

My height and weight

support my credbility with

family and friends. 


\section{Practice and Application of Knowledge by Nutrition Students}

\section{Thank you!}

Thank you for completing this survey! If you would like to be entered into a drawing for a $\$ 50$ gift card, please send an $\Theta$ mail to jennifer.boyee@uky.edu.

Thanks so much!

- Jenny 


\title{
Appendix B: Scope of Dietetics Practice Framework
}

\author{
American Dietetic Association \\ Scope of Dietetics Practice Framework
}

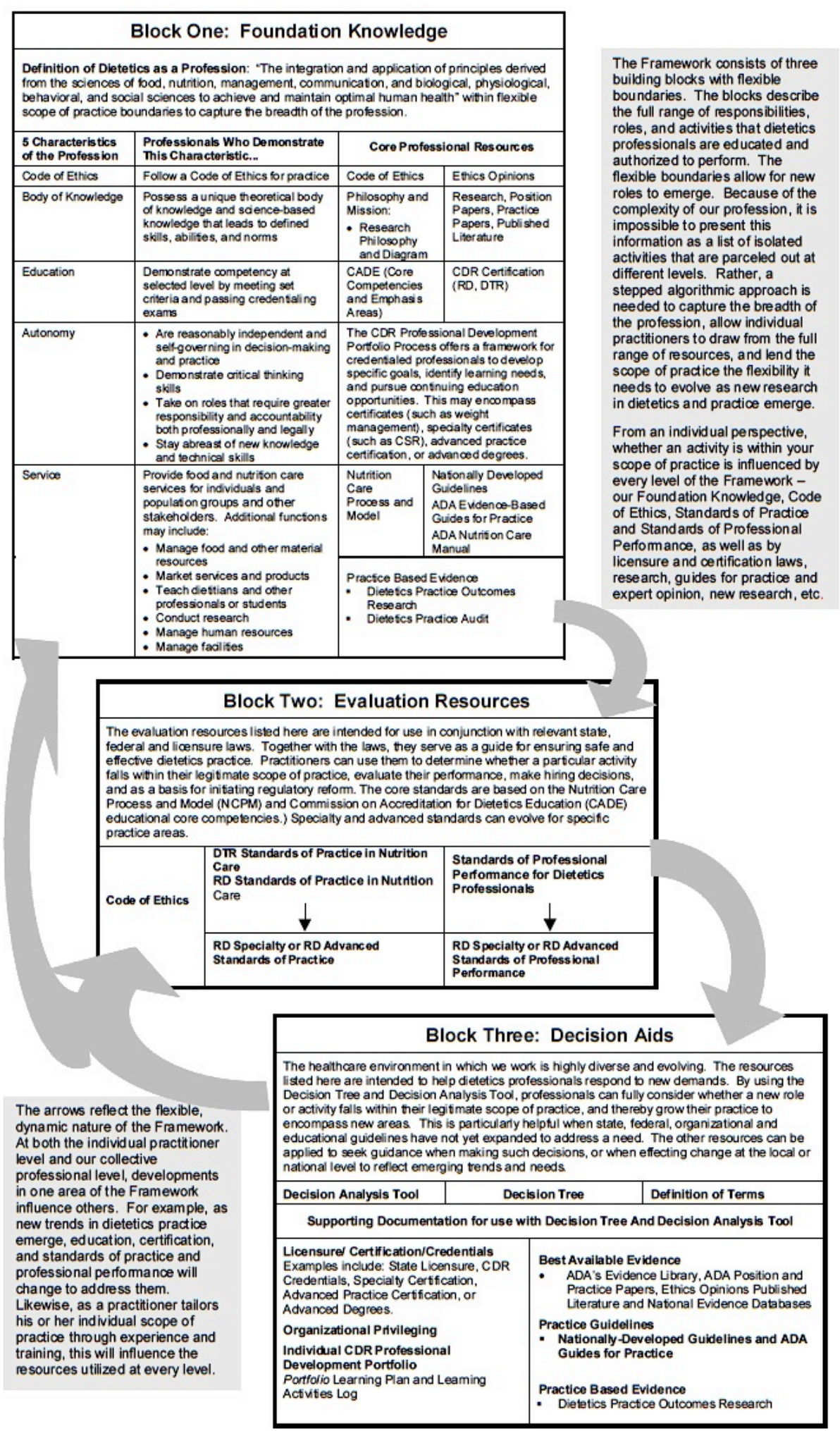




\section{Appendix C: IRB Approval Documentation}

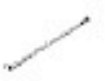

\section{UK \\ voxiventiter of \\ KENTUCKY}

Consent Form

Approval Ends

November 21, 2011
Office of Resexych Integity

IRB, IACUC, RDRC

315 Kinkesd Hall

Lexingron, KY 40506-0057

$859257-9428$

fax 859257.8995

Modification Review

Jennifer Boyee

Nutrition \& Food Science

209 Funkhouser

0054

PI phone \#: (937)307-6862

FROM: Chairperson/Vice Chairperson

Institutional Review Board (IRB)

SUBJECT: $\quad$ Approval of Modification Request for Protocol 10-0785-P4S

DATE:

December 9,2010

On December 8, 2010, the Institutional Review Board approved your request for modifications in your protocol entitled:

Practice and Application of Knowiedge by Nutrition Students

If your modification request necessitated a change in your approved informed consent/assent form(s), attached is the new IRB approved consent/assent form(s) to be used when enrolling subjects. [Note, subjects can only be enrolled using informed consent/assent forms which have a valid "IRB Approval" stamp, unless waiver from this requirement was granted by the IRB.

For information describing investigator responsibilities after obtaining IRB approval, download and read the document "PI Guidance to Responsibilities, Qualifications, Records and Documentation of Human Subjects Research" from the Office of

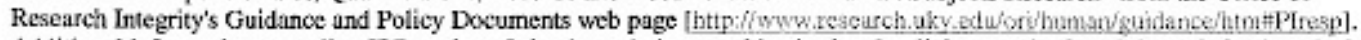
Additional information regarding IRB review, federal regulations, and institutional policies muy be found through ORI's web site [bth:l/www resarch.uky odwor]. If you have questions, need additional information, or would like a paper copy of the above mentioned document, contact the Office of Research Integrity at (859) 257-9428.

Loman Van Tharye Plolde

Chairperson/Vice Chairperson 


\section{Appendix D: Statistical Analysis of Survey Results}

Table 5 (Part A): SOLICITED ADVICE: Eat a high protein, low carbohydrate diet

\begin{tabular}{|c|c|c|c|c|c|c|c|}
\hline \multicolumn{8}{|c|}{ SOLICITED ADVICE: Eat a high protein, low carbohydrate diet } \\
\hline \multirow{5}{*}{\begin{tabular}{|l} 
Parameter \\
I only deliver \\
information \\
allowed at the \\
student level by \\
the CDR, as \\
specified in the \\
Dietetic Scope of \\
Practice. \\
\end{tabular}} & \multirow{2}{*}{$\begin{array}{l}\text { Response } \\
\text { Rarely }\end{array}$} & \multirow{2}{*}{\begin{tabular}{|r} 
Estimate \\
-1.3024
\end{tabular}} & \multirow{2}{*}{$\begin{array}{l}\text { Standard } \\
\text { Error } \\
2.3139\end{array}$} & \multicolumn{2}{|c|}{$\begin{array}{l}\text { Wald 95\% } \\
\text { Confidence Limits }\end{array}$} & \multirow{2}{*}{$\begin{array}{l}\text { Wald } \\
\text { Chi-Square } \\
\quad 0.32\end{array}$} & \multirow{2}{*}{$\begin{array}{l}\begin{array}{l}\text { Pr }> \\
\text { ChiSq }\end{array} \\
0.5735\end{array}$} \\
\hline & & & & -5.8375 & 3.2327 & & \\
\hline & Sometimes & -1.4934 & 0.9476 & -3.3507 & 0.3638 & 2.48 & 0.1150 \\
\hline & Often & -1.1857 & 0.9358 & -3.0198 & 0.6484 & 1.61 & 0.2051 \\
\hline & Always & 0.0000 & 0.0000 & 0.0000 & 0.0000 & & \\
\hline \multirow{5}{*}{$\begin{array}{l}\text { I comprehend the } \\
\text { scope of practice. }\end{array}$} & Never & -21.7956 & 93106.74 & -182508 & 182464.1 & 0.00 & 0.9998 \\
\hline & Rarely & 2.4907 & 1.9143 & -1.2613 & 6.2427 & 1.69 & 0.1932 \\
\hline & Sometimes & 1.9587 & 1.0561 & -0.1112 & 4.0286 & 3.44 & 0.0636 \\
\hline & Often & 1.8003 & 0.9599 & -0.0811 & 3.6816 & 3.52 & 0.0607 \\
\hline & Always & 0.0000 & 0.0000 & 0.0000 & 0.0000 & & \\
\hline \multirow{5}{*}{$\begin{array}{l}\text { I refer friends and } \\
\text { family to other } \\
\text { sources that are } \\
\text { outside my scope } \\
\text { of practice. }\end{array}$} & Never & 1.6359 & 2.0263 & -2.3357 & 5.6074 & 0.65 & 0.4195 \\
\hline & Rarely & -1.6626 & 1.8116 & -5.2133 & 1.8880 & 0.84 & 0.3587 \\
\hline & Sometimes & 1.2424 & 0.7975 & -0.3208 & 2.8055 & 2.43 & 0.1193 \\
\hline & Often & 0.2647 & 0.6967 & -1.1008 & 1.6302 & 0.14 & 0.7040 \\
\hline & Always & 0.0000 & 0.0000 & 0.0000 & 0.0000 & & \\
\hline \multirow{4}{*}{$\begin{array}{l}\text { I abide by the } \\
\text { Dietetics Code of } \\
\text { Ethics. }\end{array}$} & Rarely & 1.8048 & 2.3896 & -2.8788 & 6.4883 & 0.57 & 0.4501 \\
\hline & Sometimes & -0.9683 & 0.8226 & -2.5806 & 0.6440 & 1.39 & 0.2391 \\
\hline & Often & 0.0656 & 0.6421 & -1.1928 & 1.3241 & 0.01 & 0.9186 \\
\hline & Always & 0.0000 & 0.0000 & 0.0000 & 0.0000 & & \\
\hline \multirow{5}{*}{$\begin{array}{l}\text { I use nutrition } \\
\text { information to } \\
\text { diagnose health } \\
\text { conditions in } \\
\text { family members } \\
\text { and individuals. }\end{array}$} & Never & -3.7320 & 1.4233 & -6.5216 & -0.9425 & 6.88 & 0.0087 \\
\hline & Rarely & -3.7300 & 1.0057 & -5.7012 & -1.7588 & 13.75 & 0.0002 \\
\hline & Sometimes & -1.9854 & 0.8946 & -3.7387 & -0.2321 & 4.93 & 0.0265 \\
\hline & Often & -0.6999 & 0.9194 & -2.5018 & 1.1020 & 0.58 & 0.4465 \\
\hline & Always & 0.0000 & 0.0000 & 0.0000 & 0.0000 & & \\
\hline \multirow{2}{*}{ Major } & Dietetics & 0.9855 & 0.5573 & -0.1067 & 2.0778 & 3.13 & 0.0770 \\
\hline & Human Ntr & 0.0000 & 0.0000 & 0.0000 & 0.0000 & & \\
\hline
\end{tabular}


Table 5 (Part B): SOLICITED ADVICE: Eat a high protein, low carbohydrate diet

\begin{tabular}{|l|r|r|}
\hline \multicolumn{3}{|c|}{ SOLICITED ADVICE: Eat a high protein, low carbohydrate diet } \\
\hline Source & Chi-Square & Pr > ChiSq \\
\hline $\begin{array}{l}\text { I only deliver information allowed at the student level by the CDR, as } \\
\text { specified in the Dietetic Scope of Practice. }\end{array}$ & 2.56 & 0.4646 \\
\hline $\begin{array}{l}\text { I comprehend the scope of practice. } \\
\text { I refer friends and family to other sources that are outside my scope of } \\
\text { practice. }\end{array}$ & 5.37 & 0.1790 \\
\hline $\begin{array}{l}\text { I abide by the Dietetics Code of Ethics. } \\
\text { I use nutrition information to diagnose health conditions in family } \\
\text { members and individuals. }\end{array}$ & 3.71 & 0.2512 \\
\hline Major & 28.37 & $<.2944$ \\
\hline
\end{tabular}


Table 6 (Part A): SOLICITED ADVICE: Get at least 60 minutes of exercise per day, most days of the week.

\begin{tabular}{|c|c|c|c|c|c|c|c|}
\hline \multicolumn{8}{|c|}{ SOLICITED ADVICE: Get at least 60 minutes of exercise per day, most days of the week. } \\
\hline \multirow{5}{*}{$\begin{array}{l}\text { Parameter } \\
\text { I only deliver } \\
\text { information allowed } \\
\text { at the student level } \\
\text { by the CDR, as } \\
\text { specified in the } \\
\text { Dietetic Scope of } \\
\text { Practice. }\end{array}$} & \multirow{2}{*}{\begin{tabular}{l|} 
Response \\
Rarely
\end{tabular}} & \multirow{2}{*}{\begin{tabular}{|r|} 
Estimate \\
3.4983 \\
\end{tabular}} & \multirow{2}{*}{\begin{tabular}{|r|}
$\begin{array}{l}\text { Standard } \\
\text { Error }\end{array}$ \\
2.1550 \\
\end{tabular}} & \multicolumn{2}{|c|}{$\begin{array}{l}\text { Wald } 95 \% \\
\text { Confidence Limits }\end{array}$} & \multirow{2}{*}{\begin{tabular}{|r|}
$\begin{array}{l}\text { Wald } \\
\text { Chi-Square }\end{array}$ \\
2.64
\end{tabular}} & \multirow{2}{*}{\begin{tabular}{|l} 
Pr $>$ \\
ChiSq
\end{tabular}} \\
\hline & & & & -0.7254 & 7.7219 & & \\
\hline & Sometimes & -1.2602 & 0.8943 & -3.0131 & 0.4927 & 1.99 & 0.1588 \\
\hline & Often & -1.2415 & 0.8796 & -2.9655 & 0.4824 & 1.99 & 0.1581 \\
\hline & Always & 0.0000 & 0.0000 & 0.0000 & 0.0000 & & \\
\hline \multirow{5}{*}{$\begin{array}{l}\text { I comprehend the } \\
\text { scope of practice. }\end{array}$} & Never & -4.1420 & 1.7887 & -7.6478 & -0.6362 & 5.36 & 0.0206 \\
\hline & Rarely & -4.5811 & 1.3552 & -7.2373 & -1.9248 & 11.43 & 0.0007 \\
\hline & Sometimes & -0.7391 & 0.8403 & -2.3862 & 0.9079 & 0.77 & 0.3791 \\
\hline & Often & -1.4321 & 0.7724 & -2.9459 & 0.0816 & 3.44 & 0.0637 \\
\hline & Always & 0000 & 0.0000 & 0.0000 & 0.0000 & & \\
\hline \multirow{5}{*}{$\begin{array}{l}\text { I refer friends and } \\
\text { family to other } \\
\text { sources that are } \\
\text { outside my scope of } \\
\text { practice. }\end{array}$} & Never & 0.6076 & 1.9382 & -3.1912 & 4.4065 & 0.10 & 0.7539 \\
\hline & Rarely & 2.8659 & 1.7120 & -0.4895 & 6.2214 & 2.80 & 0.0941 \\
\hline & Sometimes & -0.1613 & 0.6833 & -1.5006 & 1.1779 & 0.06 & 0.8133 \\
\hline & Often & 0.4035 & 0.6213 & -0.8142 & 1.6213 & 0.42 & 0.5160 \\
\hline & Always & 0.0000 & 0.0000 & 0.0000 & 0.0000 & & \\
\hline \multirow{4}{*}{$\begin{array}{l}\text { I abide by the } \\
\text { Dietetics Code of } \\
\text { Ethics. }\end{array}$} & rely & -7.1649 & 2.5322 & -12.1280 & -2.2018 & 8.01 & 0.0047 \\
\hline & Sometimes & -0.5012 & 0.8160 & -2.1005 & 1.0982 & 0.38 & 0.5391 \\
\hline & Often & -0.7198 & 0.6535 & -2.0006 & 0.5610 & 1.21 & 0.2707 \\
\hline & Always & 0.0000 & 0.0000 & 0.0000 & 0.0000 & & \\
\hline \multirow{5}{*}{$\begin{array}{l}\text { I use nutrition } \\
\text { information to } \\
\text { diagnose health } \\
\text { conditions in family } \\
\text { members and } \\
\text { individuals. }\end{array}$} & Never & 0.8942 & 1.1325 & -1.3255 & 3.1138 & 0.62 & 0.4298 \\
\hline & Rarely & -0.3734 & 0.7864 & -1.9148 & 1.1680 & 0.23 & 0.6349 \\
\hline & Sometimes & -0.5889 & 0.7894 & -2.1362 & 0.9584 & 0.56 & 0.4557 \\
\hline & Often & 0.3349 & 0.8370 & -1.3056 & 1.9754 & 0.16 & 0.6891 \\
\hline & Always & 0.0000 & 0.0000 & 0.0000 & 0.0000 & & \\
\hline \multirow{2}{*}{ Major } & Dietetics & -0.2344 & 0.5139 & -1.2417 & 0.7729 & 0.21 & 0.6483 \\
\hline & Human Ntr & 0.0000 & 0.0000 & 0.0000 & 0.0000 & & \\
\hline
\end{tabular}


Table 6 (Part B): SOLICITED ADVICE: Get at least 60 minutes of exercise per day, most days of the week.

\begin{tabular}{|l|r|r|}
\hline \multicolumn{2}{|c|}{ SOLICITED ADVICE: Get at least 60 minutes of exercise per day, most days of the week. } \\
\hline Source & Chi-Square & Pr > ChiSq \\
\hline $\begin{array}{l}\text { I only deliver information allowed at the student level by the CDR, as } \\
\text { specified in the Dietetic Scope of Practice. }\end{array}$ & 6.57 & 0.0870 \\
\hline $\begin{array}{l}\text { I comprehend the scope of practice. } \\
\text { I refer friends and family to other sources that are outside my scope of } \\
\text { practice. }\end{array}$ & 17.49 & 0.0015 \\
\hline $\begin{array}{l}\text { I abide by the Dietetics Code of Ethics. } \\
\text { I use nutrition information to diagnose health conditions in family } \\
\text { members and individuals. }\end{array}$ & 10.53 & 0.4306 \\
\hline \begin{tabular}{l} 
Major \\
\hline
\end{tabular} & 4.35 & 0.0146 \\
\hline
\end{tabular}


Table 7 (Part A): SOLICITED ADVICE: Eat several smaller meals throughout the day instead of 3 large meals.

\begin{tabular}{|c|c|c|c|c|c|c|c|}
\hline \multirow{5}{*}{$\begin{array}{l}\text { Parameter } \\
\text { I only deliver } \\
\text { information allowed } \\
\text { at the student level } \\
\text { by the CDR, as } \\
\text { specified in the } \\
\text { Dietetic Scope of } \\
\text { Practice. }\end{array}$} & \multirow{2}{*}{\begin{tabular}{|l|} 
Response \\
Rarely
\end{tabular}} & \multirow{2}{*}{$\begin{array}{r}\text { Estimate } \\
-2.4809\end{array}$} & \multirow{2}{*}{\begin{tabular}{|r|}
$\begin{array}{l}\text { Standard } \\
\text { Error }\end{array}$ \\
1.8426
\end{tabular}} & \multicolumn{2}{|c|}{$\begin{array}{l}\text { Wald 95\% } \\
\text { Confidence Limits }\end{array}$} & \multirow{2}{*}{$\begin{array}{l}\text { Wald } \\
\text { Chi-Square } \\
1.81\end{array}$} & \multirow{2}{*}{ 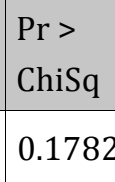 } \\
\hline & & & & -6.0923 & 1.1305 & & \\
\hline & Sometimes & 0.3209 & 0.8370 & -1.3197 & 1.9614 & 0.15 & 0.7015 \\
\hline & Often & 0.0388 & 0.8335 & -1.5947 & 1.6724 & 0.00 & 0.9628 \\
\hline & Always & 0.0000 & 0.0000 & 0.0000 & 0.0000 & & \\
\hline \multirow{5}{*}{$\begin{array}{l}\text { I comprehend the } \\
\text { scope of practice. }\end{array}$} & Never & 23.6663 & 83608.96 & -163847 & 163894.2 & 0.00 & 0.9998 \\
\hline & Rarely & -0.3481 & 1.2486 & -2.7953 & 2.0991 & 0.08 & 0.7804 \\
\hline & Sometimes & 1.3508 & 0.8490 & -0.3133 & 3.0149 & 2.53 & 0.1116 \\
\hline & Often & 0.7309 & 0.7503 & -0.7397 & 2.2016 & 0.95 & 0.3300 \\
\hline & Always & 0.0000 & 0.0000 & 0.0000 & 0.0000 & & \\
\hline \multirow{5}{*}{$\begin{array}{l}\text { I refer friends and } \\
\text { family to other } \\
\text { sources that are } \\
\text { outside my scope of } \\
\text { practice. }\end{array}$} & Never & 0.5676 & 1.9314 & -3.2179 & 4.3531 & 0.09 & 0.7689 \\
\hline & Rarely & 2.0225 & 1.7032 & -1.3158 & 5.3608 & 1.41 & 0.2350 \\
\hline & Sometimes & -0.9604 & 0.6640 & -2.2618 & 0.3410 & 2.09 & 0.1481 \\
\hline & Often & -0.6611 & 0.5800 & -1.7980 & 0.4757 & 1.30 & 0.2544 \\
\hline & Always & 0.0000 & 0.0000 & 0.0000 & 0.0000 & & \\
\hline \multirow{4}{*}{$\begin{array}{l}\text { I abide by the } \\
\text { Dietetics Code of } \\
\text { Ethics. }\end{array}$} & Rarely & 1.3848 & 1.8958 & -2.3309 & 5.1005 & 0.53 & 0.4651 \\
\hline & Sometimes & -1.1729 & 0.7805 & -2.7027 & 0.3568 & 2.26 & 0.1329 \\
\hline & Often & -1.1911 & 0.6426 & -2.4505 & 0.0684 & 3.44 & 0.0638 \\
\hline & Always & 0.0000 & 0.0000 & 0.0000 & 0.0000 & & \\
\hline \multirow{5}{*}{$\begin{array}{l}\text { I use nutrition } \\
\text { information to } \\
\text { diagnose health } \\
\text { conditions in family } \\
\text { members and } \\
\text { individuals. }\end{array}$} & Never & 0.1818 & 1.0812 & -1.9373 & 2.3010 & 0.03 & 0.8664 \\
\hline & Rarely & 0.6232 & 0.7778 & -0.9013 & 2.1476 & 0.64 & 0.4230 \\
\hline & Sometimes & 0.7016 & 0.7659 & -0.7996 & 2.2028 & 0.84 & 0.3596 \\
\hline & Often & 0.5803 & 0.8363 & -1.0589 & 2.2195 & 0.48 & 0.4878 \\
\hline & Always & 0.0000 & 0.0000 & 0.0000 & 0.0000 & & \\
\hline \multirow{2}{*}{ Major } & Dietetics & 0.4346 & 0.4956 & -0.5367 & 1.4059 & 0.77 & 0.3805 \\
\hline & Human Ntr & 0.0000 & 0.0000 & 0.0000 & 0.0000 & & \\
\hline
\end{tabular}


Table 7 (Part B): SOLICITED ADVICE: Eat several smaller meals throughout the day instead of 3 large meals.

SOLICITED ADVICE: Eat several smaller meals throughout the day instead of 3 large meals.

\begin{tabular}{|l|r|r|}
\hline Source & Chi-Square & Pr > ChiSq \\
\hline $\begin{array}{l}\text { I only deliver information allowed at the student level by the CDR, as } \\
\text { specified in the Dietetic Scope of Practice. }\end{array}$ & 2.84 & 0.4174 \\
\hline I comprehend the scope of practice. & 5.85 & 0.2108 \\
\hline $\begin{array}{l}\text { I refer friends and family to other sources that are outside my scope } \\
\text { of practice. }\end{array}$ & 5.30 & 0.2578 \\
\hline I abide by the Dietetics Code of Ethics. & 4.44 & 0.2175 \\
\hline $\begin{array}{l}\text { I use nutrition information to diagnose health conditions in family } \\
\text { members and individuals. }\end{array}$ & 1.06 & 0.9007 \\
\hline Major & 0.77 & 0.3804 \\
\hline
\end{tabular}


Table 8 (Part A): SOLICITED ADVICE: Avoid eating or drinking anything after dinner.

\begin{tabular}{|c|c|c|c|c|c|c|c|}
\hline \multicolumn{8}{|c|}{ SOLICITED ADVICE: Avoid eating or drinking anything after dinner. } \\
\hline \multirow{5}{*}{$\begin{array}{l}\text { Parameter } \\
\text { I only deliver } \\
\text { information } \\
\text { allowed at the } \\
\text { student level by the } \\
\text { CDR, as specified in } \\
\text { the Dietetic Scope } \\
\text { of Practice. }\end{array}$} & \multirow{2}{*}{$\begin{array}{l}\text { Response } \\
\text { Rarely }\end{array}$} & \multirow{2}{*}{$\begin{array}{r}\text { Estimate } \\
2.7029\end{array}$} & \multirow{2}{*}{\begin{tabular}{|l|}
$\begin{array}{l}\text { Standard } \\
\text { Error }\end{array}$ \\
2.4274
\end{tabular}} & \multicolumn{2}{|c|}{$\begin{array}{l}\text { Wald 95\% } \\
\text { Confidence Limits }\end{array}$} & \multirow{2}{*}{\begin{tabular}{|r}
$\begin{array}{l}\text { Wald } \\
\text { Chi-Square }\end{array}$ \\
1.24
\end{tabular}} & \multirow{2}{*}{$\begin{array}{l}\begin{array}{l}\mathrm{Pr}> \\
\text { ChiSq }\end{array} \\
0.2655\end{array}$} \\
\hline & & & & -2.0547 & 7.4605 & & \\
\hline & Sometimes & -0.8208 & 0.8848 & -2.5550 & 0.9135 & 0.86 & 0.3536 \\
\hline & Often & -1.8610 & 0.9621 & -3.7467 & 0.0247 & 3.74 & 0.0531 \\
\hline & Always & 0.0000 & 0.0000 & 0.0000 & 0.0000 & & \\
\hline \multirow{5}{*}{$\begin{array}{l}\text { I comprehend the } \\
\text { scope of practice. }\end{array}$} & Never & -21.6486 & 89473.68 & -175387 & 175343.5 & 0.00 & 0.9998 \\
\hline & Rarely & -0.4064 & 1.4899 & -3.3265 & 2.5137 & 0.07 & 0.7850 \\
\hline & Sometimes & 2.1148 & 1.0096 & 0.1360 & 4.0937 & 4.39 & 0.0362 \\
\hline & Often & 2.2329 & 0.9717 & 0.3284 & 4.1374 & 5.28 & 0.0216 \\
\hline & Always & 0.0000 & 0.0000 & 0.0000 & 0.0000 & & \\
\hline \multirow{5}{*}{$\begin{array}{l}\text { I refer friends and } \\
\text { family to other } \\
\text { sources that are } \\
\text { outside my scope } \\
\text { of practice. }\end{array}$} & Never & 1.1822 & 1.8527 & -2.4490 & 4.8134 & 0.41 & 0.5234 \\
\hline & Rarely & 4.0807 & 1.6173 & 0.9108 & 7.2506 & 6.37 & 0.0116 \\
\hline & Sometimes & 0.8731 & 0.7206 & -0.5393 & 2.2855 & 1.47 & 0.2257 \\
\hline & Often & 0.7572 & 0.6035 & -0.4257 & 1.9401 & 1.57 & 0.2096 \\
\hline & Always & 0.0000 & 0.0000 & 0.0000 & 0.0000 & & \\
\hline \multirow{4}{*}{$\begin{array}{l}\text { I abide by the } \\
\text { Dietetics Code of } \\
\text { Ethics. }\end{array}$} & Rarely & -4.1856 & 2.5289 & -9.1422 & 0.7710 & 2.74 & 0.0979 \\
\hline & Sometimes & -0.3869 & 0.7939 & -1.9430 & 1.1691 & 0.24 & 0.6260 \\
\hline & Often & 0.1576 & 0.6595 & -1.1350 & 1.4501 & 0.06 & 0.8112 \\
\hline & Always & 0.0000 & 0.0000 & 0.0000 & 0.0000 & & \\
\hline \multirow{5}{*}{$\begin{array}{l}\text { I use nutrition } \\
\text { information to } \\
\text { diagnose health } \\
\text { conditions in } \\
\text { family members } \\
\text { and individuals. }\end{array}$} & Never & -2.0142 & 1.1271 & -4.2233 & 0.1948 & 3.19 & 0.0739 \\
\hline & Rarely & -2.1412 & 0.8184 & -3.7451 & -0.5372 & 6.85 & 0.0089 \\
\hline & Sometimes & -2.0659 & 0.8242 & -3.6813 & -0.4505 & 6.28 & 0.0122 \\
\hline & Often & -0.6311 & 0.8108 & -2.2201 & 0.9580 & 0.61 & 0.4363 \\
\hline & Always & 0.0000 & 0.0000 & 0.0000 & 0.0000 & & \\
\hline \multirow{2}{*}{ Major } & Dietetics & 0.7337 & 0.5175 & -0.2806 & 1.7480 & 2.01 & 0.1563 \\
\hline & Human Ntr & 0.0000 & 0.0000 & 0.0000 & 0.0000 & & \\
\hline
\end{tabular}


Table 8 (Part B): SOLICITED ADVICE: Avoid eating or drinking anything after dinner.

SOLICITED ADVICE: Avoid eating or drinking anything after dinner.

\begin{tabular}{|l|r|r|}
\hline \multicolumn{3}{|c|}{ SOLICITED ADVICE: Avoid eating or drinking anything after dinner. } \\
\hline Source & Chi-Square & Pr > ChiSq \\
\hline $\begin{array}{l}\text { I only deliver information allowed at the student level by the CDR, as } \\
\text { specified in the Dietetic Scope of Practice. }\end{array}$ & 7.68 & 0.0531 \\
\hline I comprehend the scope of practice. & 12.15 & 0.0162 \\
\hline $\begin{array}{l}\text { I refer friends and family to other sources that are outside my scope of } \\
\text { practice. }\end{array}$ & 6.97 & 0.1374 \\
\hline $\begin{array}{l}\text { I abide by the Dietetics Code of Ethics. } \\
\text { I use nutrition information to diagnose health conditions in family } \\
\text { members and individuals. }\end{array}$ & 3.24 & 0.3563 \\
\hline Major & 14.64 & 0.0055 \\
\hline
\end{tabular}


Table 9 (Part A): SOLICITED ADVICE: Eat a high protein, high calorie diet.

\begin{tabular}{|c|c|c|c|c|c|c|c|}
\hline \multicolumn{8}{|c|}{ SOLICITED ADVICE: Eat a high protein, high calorie diet. } \\
\hline \multirow{5}{*}{$\begin{array}{l}\text { Parameter } \\
\text { I only deliver } \\
\text { information allowed } \\
\text { at the student level } \\
\text { by the CDR, as } \\
\text { specified in the } \\
\text { Dietetic Scope of } \\
\text { Practice. }\end{array}$} & \multirow{2}{*}{$\begin{array}{l}\text { Response } \\
\text { Rarely }\end{array}$} & \multirow{2}{*}{\begin{tabular}{|r} 
Estimate \\
3.7363
\end{tabular}} & \multirow{2}{*}{$\begin{array}{l}\begin{array}{l}\text { Standard } \\
\text { Error }\end{array} \\
\qquad 2.4147\end{array}$} & \multicolumn{2}{|c|}{$\begin{array}{l}\text { Wald 95\% } \\
\text { Confidence Limits }\end{array}$} & \multirow{2}{*}{\begin{tabular}{|r}
$\begin{array}{l}\text { Wald } \\
\text { Chi-Square }\end{array}$ \\
2.39
\end{tabular}} & \multirow{2}{*}{$\begin{array}{l}\begin{array}{l}\text { Pr }> \\
\text { ChiSq }\end{array} \\
0.1218\end{array}$} \\
\hline & & & & -0.9964 & 8.4690 & & \\
\hline & Sometimes & 1.1546 & 1.0975 & -0.9965 & 3.3058 & 1.11 & 0.2928 \\
\hline & Often & 0.8882 & 1.1070 & -1.2815 & 3.0579 & 0.64 & 0.4223 \\
\hline & Always & 0.0000 & 0.0000 & 0.0000 & 0.0000 & & \\
\hline \multirow{5}{*}{$\begin{array}{l}\text { I comprehend the } \\
\text { scope of practice. }\end{array}$} & Never & -22.9196 & 104388.4 & -204621 & 204574.7 & 0.00 & 0.9998 \\
\hline & Rarely & -0.0688 & 1.7922 & -3.5815 & 3.4439 & 0.00 & 0.9694 \\
\hline & Sometimes & 1.2877 & 1.1830 & -1.0309 & 3.6063 & 1.18 & 0.2764 \\
\hline & Often & 1.6630 & 1.0834 & -0.4605 & 3.7865 & 2.36 & 0.1248 \\
\hline & Always & 0.0000 & 0.0000 & 0.0000 & 0.0000 & & \\
\hline \multirow{5}{*}{$\begin{array}{l}\text { I refer friends and } \\
\text { family to other } \\
\text { sources that are } \\
\text { outside my scope of } \\
\text { practice. }\end{array}$} & Never & 3.2394 & 2.2146 & -1.1011 & 7.5800 & 2.14 & 0.1435 \\
\hline & Rarely & 0.9256 & 1.8219 & -2.6453 & 4.4966 & 0.26 & 0.6114 \\
\hline & Sometimes & 1.8499 & 0.8255 & 0.2319 & 3.4678 & 5.02 & 0.0250 \\
\hline & Often & 0.3683 & 0.7420 & -1.0861 & 1.8227 & 0.25 & 0.6197 \\
\hline & Always & 0.0000 & 0.0000 & 0.0000 & 0.0000 & & \\
\hline \multirow{4}{*}{$\begin{array}{l}\text { I abide by the } \\
\text { Dietetics Code of } \\
\text { Ethics. }\end{array}$} & Rarely & -0.8069 & 2.4625 & -5.6333 & 4.0195 & 0.11 & 0.7432 \\
\hline & Sometimes & -2.5071 & 0.9311 & -4.3320 & -0.6823 & 7.25 & 0.0071 \\
\hline & Often & -1.4096 & 0.7664 & -2.9117 & 0.0925 & 3.38 & 0.0659 \\
\hline & Always & 0.0000 & 0.0000 & 0.0000 & 0.0000 & & \\
\hline \multirow{5}{*}{$\begin{array}{l}\text { I use nutrition } \\
\text { information to } \\
\text { diagnose health } \\
\text { conditions in family } \\
\text { members and } \\
\text { individuals. }\end{array}$} & Never & -3.4948 & 1.4437 & -6.3245 & -0.6652 & 5.86 & 0.0155 \\
\hline & Rarely & -3.8806 & 1.1452 & -6.1252 & -1.6360 & 11.48 & 0.0007 \\
\hline & Sometimes & -2.0770 & 1.0189 & -4.0740 & -0.0801 & 4.16 & 0.0415 \\
\hline & Often & -0.7698 & 1.0497 & -2.8272 & 1.2876 & 0.54 & 0.4633 \\
\hline & Always & 0.0000 & 0.0000 & 0.0000 & 0.0000 & & \\
\hline \multirow{2}{*}{ Major } & Dietetics & 0.4157 & 0.6086 & -0.7771 & 1.6084 & 0.47 & 0.4946 \\
\hline & Human Ntr & 0.0000 & 0.0000 & 0.0000 & 0.0000 & & \\
\hline
\end{tabular}


Table 9 (Part B): SOLICITED ADVICE: Eat a high protein, high calorie diet.

SOLICITED ADVICE: Eat a high protein, high calorie diet.

\begin{tabular}{|l|r|r|}
\hline Source & Chi-Square & Pr > ChiSq \\
\hline $\begin{array}{l}\text { I only deliver information allowed at the student level by the CDR, as } \\
\text { specified in the Dietetic Scope of Practice. }\end{array}$ & 2.73 & 0.4359 \\
\hline I comprehend the scope of practice. & 5.87 & 0.2087 \\
\hline $\begin{array}{l}\text { I refer friends and family to other sources that are outside my scope } \\
\text { of practice. }\end{array}$ & 8.63 & 0.0712 \\
\hline I abide by the Dietetics Code of Ethics. & 7.65 & 0.0538 \\
\hline $\begin{array}{l}\text { I use nutrition information to diagnose health conditions in family } \\
\text { members and individuals. }\end{array}$ & 25.39 & $<.0001$ \\
\hline Major & 0.47 & 0.4926 \\
\hline
\end{tabular}


Table 10 (Part A): SOLICITED ADVICE: Avoid calorically dense foods and beverages.

\begin{tabular}{|c|c|c|c|c|c|c|c|}
\hline \multicolumn{8}{|c|}{ SOLICITED ADVICE: Avoid calorically dense foods and beverages. } \\
\hline \multirow{5}{*}{$\begin{array}{l}\text { Parameter } \\
\text { I only deliver } \\
\text { information allowed at } \\
\text { the student level by } \\
\text { the CDR, as specified in } \\
\text { the Dietetic Scope of } \\
\text { Practice. }\end{array}$} & \multirow{2}{*}{$\begin{array}{l}\text { Response } \\
\text { Rarely }\end{array}$} & \multirow{2}{*}{\begin{tabular}{|r|} 
Estimate \\
-0.2687
\end{tabular}} & \multirow{2}{*}{\begin{tabular}{|l|}
$\begin{array}{l}\text { Standard } \\
\text { Error }\end{array}$ \\
1.8681
\end{tabular}} & \multicolumn{2}{|c|}{$\begin{array}{l}\text { Wald 95\% } \\
\text { Confidence Limits }\end{array}$} & \multirow{2}{*}{\begin{tabular}{|r|}
$\begin{array}{l}\text { Wald } \\
\text { Chi-Square }\end{array}$ \\
0.02 \\
\end{tabular}} & \multirow{2}{*}{\begin{tabular}{|l|}
$\begin{array}{l}\mathrm{Pr}> \\
\text { ChiSq }\end{array}$ \\
0.8856
\end{tabular}} \\
\hline & & & & -3.9301 & 3.3927 & & \\
\hline & Sometimes & 0.2540 & 0.9489 & -1.6058 & 2.1137 & 0.07 & 0.7890 \\
\hline & Often & 0.0068 & 0.9458 & -1.8470 & 1.8607 & 0.00 & 0.9942 \\
\hline & Always & 0.0000 & 0.0000 & 0.0000 & 0.0000 & & \\
\hline \multirow{5}{*}{$\begin{array}{l}\text { I comprehend the } \\
\text { scope of practice. }\end{array}$} & Never & 1.3812 & 1.9105 & -2.3634 & 5.1258 & 0.52 & 0.4697 \\
\hline & Rarely & 0.7353 & 1.3008 & -1.8141 & 3.2848 & 0.32 & 0.5719 \\
\hline & Sometimes & 0.0991 & 0.8095 & -1.4875 & 1.6856 & 0.01 & 0.9026 \\
\hline & Often & -0.1480 & 0.7542 & -1.6261 & 1.3302 & 0.04 & 0.8445 \\
\hline & Always & 0.0000 & 0.0000 & 0.0000 & 0.0000 & & \\
\hline \multirow{5}{*}{$\begin{array}{l}\text { I refer friends and } \\
\text { family to other sources } \\
\text { that are outside my } \\
\text { scope of practice. }\end{array}$} & Never & 1.3962 & 2.0098 & -2.5430 & 5.3355 & 0.48 & 0.4872 \\
\hline & Rarely & 1.0153 & 1.6003 & -2.1213 & 4.1518 & 0.40 & 0.5258 \\
\hline & Sometimes & -0.4954 & 0.6843 & -1.8366 & 0.8459 & 0.52 & 0.4691 \\
\hline & Often & -0.3520 & 0.5946 & -1.5173 & 0.8133 & 0.35 & 0.5538 \\
\hline & Always & 0.0000 & 0.0000 & 0.0000 & 0.0000 & & \\
\hline \multirow{4}{*}{$\begin{array}{l}\text { I abide by the Dietetics } \\
\text { Code of Ethics. }\end{array}$} & Rarely & 0.6977 & 2.0340 & -3.2889 & 4.6843 & 0.12 & 0.7316 \\
\hline & Sometimes & -0.6391 & 0.7359 & -2.0814 & 0.8032 & 0.75 & 0.3851 \\
\hline & Often & -0.4499 & 0.6168 & -1.6588 & 0.7591 & 0.53 & 0.4658 \\
\hline & Always & 0.0000 & 0.0000 & 0.0000 & 0.0000 & & \\
\hline \multirow{5}{*}{$\begin{array}{l}\text { I use nutrition } \\
\text { information to } \\
\text { diagnose health } \\
\text { conditions in family } \\
\text { members and } \\
\text { individuals. }\end{array}$} & Never & -1.6640 & 1.0966 & -3.8133 & 0.4854 & 2.30 & 0.1292 \\
\hline & Rarely & -2.0328 & 0.8736 & -3.7450 & -0.3206 & 5.41 & 0.0200 \\
\hline & Sometimes & -2.1419 & 0.8671 & -3.8414 & -0.4423 & 6.10 & 0.0135 \\
\hline & Often & -1.5354 & 0.9123 & -3.3234 & 0.2526 & 2.83 & 0.0924 \\
\hline & Always & 0.0000 & 0.0000 & 0.0000 & 0.0000 & & \\
\hline \multirow{2}{*}{ Major } & Dietetics & 0.2283 & 0.5180 & -0.7869 & 1.2436 & 0.19 & 0.6594 \\
\hline & Human Ntr & 0.0000 & 0.0000 & 0.0000 & 0.0000 & & \\
\hline
\end{tabular}


Table 10 (Part B): SOLICITED ADVICE: Avoid calorically dense foods and beverages.

\begin{tabular}{|l|r|r|}
\hline \multicolumn{3}{|c|}{ SOLICITED ADVICE: Avoid calorically dense foods and beverages. } \\
\hline Source & Chi-Square & Pr > ChiSq \\
\hline $\begin{array}{l}\text { I only deliver information allowed at the student level by the CDR, as } \\
\text { specified in the Dietetic Scope of Practice. }\end{array}$ & 0.29 & 0.9611 \\
\hline $\begin{array}{l}\text { I comprehend the scope of practice. } \\
\text { I refer friends and family to other sources that are outside my scope of } \\
\text { practice. }\end{array}$ & 2.04 & 0.8679 \\
\hline $\begin{array}{l}\text { I abide by the Dietetics Code of Ethics. } \\
\text { I use nutrition information to diagnose health conditions in family } \\
\text { members and individuals. }\end{array}$ & 1.07 & 0.7843 \\
\hline Major & 0.46 & 0.1136 \\
\hline
\end{tabular}


Table 11 (Part A): SOLICITED ADVICE: Consume less alcoholic beverages.

\begin{tabular}{|c|c|c|c|c|c|c|c|}
\hline \multicolumn{8}{|c|}{ SOLICITED ADVICE: Consume less alcoholic beverages. } \\
\hline \multirow{5}{*}{$\begin{array}{l}\text { Parameter } \\
\text { I only deliver } \\
\text { information allowed at } \\
\text { the student level by } \\
\text { the CDR, as specified in } \\
\text { the Dietetic Scope of } \\
\text { Practice. }\end{array}$} & \multirow{2}{*}{$\begin{array}{l}\text { Response } \\
\text { Rarely }\end{array}$} & \multirow{2}{*}{\begin{tabular}{|r} 
Estimate \\
-0.8841 \\
\end{tabular}} & \multirow{2}{*}{\begin{tabular}{|r|}
$\begin{array}{l}\text { Standard } \\
\text { Error }\end{array}$ \\
1.7765
\end{tabular}} & \multicolumn{2}{|c|}{$\begin{array}{l}\text { Wald 95\% } \\
\text { Confidence Limits }\end{array}$} & \multirow{2}{*}{\begin{tabular}{|r}
$\begin{array}{l}\text { Wald } \\
\text { Chi-Square }\end{array}$ \\
0.25
\end{tabular}} & \multirow{2}{*}{$\begin{array}{l}\text { Pr }> \\
\text { ChiSq } \\
0.6187\end{array}$} \\
\hline & & & & -4.3660 & 2.5978 & & \\
\hline & Sometimes & -0.1843 & 0.8030 & -1.7582 & 1.3895 & 0.05 & 0.8185 \\
\hline & Often & -0.9175 & 0.8008 & -2.4870 & 0.6520 & 1.31 & 0.2519 \\
\hline & Always & 0.0000 & 0.0000 & 0.0000 & 0.0000 & & \\
\hline \multirow{5}{*}{$\begin{array}{l}\text { I comprehend the } \\
\text { scope of practice. }\end{array}$} & Never & 0.1144 & 1.7350 & -3.2861 & 3.5149 & 0.00 & 0.9474 \\
\hline & Rarely & 0.6169 & 1.3974 & -2.1220 & 3.3558 & 0.19 & 0.6589 \\
\hline & Sometimes & 1.0035 & 0.7810 & -0.5272 & 2.5342 & 1.65 & 0.1988 \\
\hline & Often & 1.4644 & 0.7135 & 0.0659 & 2.8628 & 4.21 & 0.0401 \\
\hline & Always & 0.0000 & 0.0000 & 0.0000 & 0.0000 & & \\
\hline \multirow{5}{*}{$\begin{array}{l}\text { I refer friends and } \\
\text { family to other sources } \\
\text { that are outside my } \\
\text { scope of practice. }\end{array}$} & Never & 1.9245 & 1.9438 & -1.8852 & 5.7342 & 0.98 & 0.3221 \\
\hline & Rarely & 0.2317 & 1.5275 & -2.7621 & 3.2256 & 0.02 & 0.8794 \\
\hline & Sometimes & 0.5744 & 0.6574 & -0.7140 & 1.8628 & 0.76 & 0.3822 \\
\hline & Often & -0.1368 & 0.5618 & -1.2380 & 0.9644 & 0.06 & 0.8077 \\
\hline & Always & 0.0000 & 0.0000 & 0.0000 & 0.0000 & & \\
\hline \multirow{4}{*}{$\begin{array}{l}\text { I abide by the Dietetics } \\
\text { Code of Ethics. }\end{array}$} & Rarely & 0.2733 & 2.4469 & -4.5224 & 5.0691 & 0.01 & 0.9111 \\
\hline & Sometimes & -1.7281 & 0.7293 & -3.1575 & -0.2986 & 5.61 & 0.0178 \\
\hline & Often & -0.8805 & 0.5755 & -2.0085 & 0.2474 & 2.34 & 0.1260 \\
\hline & Always & 0.0000 & 0.0000 & 0.0000 & 0.0000 & & \\
\hline \multirow{5}{*}{$\begin{array}{l}\text { I use nutrition } \\
\text { information to } \\
\text { diagnose health } \\
\text { conditions in family } \\
\text { members and } \\
\text { individuals. }\end{array}$} & Never & -0.4090 & 1.0588 & -2.4843 & 1.6663 & 0.15 & 0.6993 \\
\hline & Rarely & -0.7612 & 0.7944 & -2.3183 & 0.7958 & 0.92 & 0.3380 \\
\hline & Sometimes & -0.6268 & 0.7508 & -2.0983 & 0.8447 & 0.70 & 0.4038 \\
\hline & Often & 0.5397 & 0.8392 & -1.1050 & 2.1845 & 0.41 & 0.5201 \\
\hline & Always & 0.0000 & 0.0000 & 0.0000 & 0.0000 & & \\
\hline \multirow{2}{*}{ Major } & Dietetics & 0.7726 & 0.5170 & -0.2407 & 1.7859 & 2.23 & 0.1351 \\
\hline & Human Ntr & 0.0000 & 0.0000 & 0.0000 & 0.0000 & & \\
\hline
\end{tabular}


Table 11 (Part B): SOLICITED ADVICE: Consume less alcoholic beverages.

\begin{tabular}{|l|r|r|}
\hline \multicolumn{3}{|c|}{ SOLICITED ADVICE: Consume less alcoholic beverages. } \\
\hline Source & Chi-Square & Pr > ChiSq \\
\hline $\begin{array}{l}\text { I only deliver information allowed at the student level by the CDR, as } \\
\text { specified in the Dietetic Scope of Practice. }\end{array}$ & 2.57 & 0.4622 \\
\hline I comprehend the scope of practice. & 4.79 & 0.3097 \\
\hline $\begin{array}{l}\text { I refer friends and family to other sources that are outside my scope of } \\
\text { practice. }\end{array}$ & 2.65 & 0.6183 \\
\hline I abide by the Dietetics Code of Ethics. & 5.82 & 0.1209 \\
\hline $\begin{array}{l}\text { I use nutrition information to diagnose health conditions in family } \\
\text { members and individuals. }\end{array}$ & 5.93 & 0.2045 \\
\hline Major & 2.26 & 0.1327 \\
\hline
\end{tabular}


Table 12 (Part A): SOLICITED ADVICE: Make smart food decisions when eating away from home.

\begin{tabular}{|c|c|c|c|c|c|c|c|}
\hline \multicolumn{8}{|c|}{ SOLICITED ADVICE: Make smart food decisions when eating away from home. } \\
\hline \multirow{5}{*}{$\begin{array}{l}\text { Parameter } \\
\text { I only deliver } \\
\text { information } \\
\text { allowed at the } \\
\text { student level by the } \\
\text { CDR, as specified in } \\
\text { the Dietetic Scope } \\
\text { of Practice. }\end{array}$} & \multirow{2}{*}{$\begin{array}{l}\text { Response } \\
\text { Rarely }\end{array}$} & \multirow{2}{*}{\begin{tabular}{r|} 
Estimate \\
1.3194
\end{tabular}} & \multirow{2}{*}{\begin{tabular}{|r|}
$\begin{array}{l}\text { Standard } \\
\text { Error }\end{array}$ \\
2.1709
\end{tabular}} & \multicolumn{2}{|c|}{$\begin{array}{l}\text { Wald 95\% } \\
\text { Confidence Limits }\end{array}$} & \multirow{2}{*}{$\begin{array}{l}\text { Wald } \\
\text { Chi-Square } \\
0.37\end{array}$} & \multirow{2}{*}{ 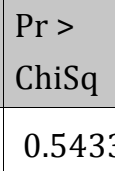 } \\
\hline & & & & -2.9355 & 5.5743 & & \\
\hline & Sometimes & 0.8009 & 0.9340 & -1.0297 & 2.6314 & 0.74 & 0.3912 \\
\hline & Often & -0.4012 & 0.9470 & -2.2572 & 1.4548 & 0.18 & 0.6718 \\
\hline & Always & 0.0000 & 0.0000 & 0.0000 & 0.0000 & & \\
\hline \multirow{5}{*}{$\begin{array}{l}\text { I comprehend the } \\
\text { scope of practice. }\end{array}$} & Never & 21.3691 & 90651.29 & -177652 & 177694.6 & 0.00 & 0.9998 \\
\hline & Rarely & -0.8028 & 1.4991 & -3.7409 & 2.1353 & 0.29 & 0.5923 \\
\hline & Sometimes & -0.5268 & 0.9555 & -2.3994 & 1.3459 & 0.30 & 0.5814 \\
\hline & Often & -0.8156 & 0.8796 & -2.5396 & 0.9084 & 0.86 & 0.3538 \\
\hline & Always & 0.0000 & 0.0000 & 0.0000 & 0.0000 & & \\
\hline \multirow{5}{*}{$\begin{array}{l}\text { I refer friends and } \\
\text { family to other } \\
\text { sources that are } \\
\text { outside my scope of } \\
\text { practice. }\end{array}$} & Never & 24.5638 & 90651.29 & -177649 & 177697.8 & 0.00 & 0.9998 \\
\hline & Rarely & 0.3266 & 1.5850 & -2.7800 & 3.4332 & 0.04 & 0.8368 \\
\hline & Sometimes & -0.2472 & 0.7153 & -1.6492 & 1.1547 & 0.12 & 0.7296 \\
\hline & Often & -0.0955 & 0.6455 & -1.3607 & 1.1696 & 0.02 & 0.8824 \\
\hline & Always & 0.0000 & 0.0000 & 0.0000 & 0.0000 & & \\
\hline \multirow{4}{*}{$\begin{array}{l}\text { I abide by the } \\
\text { Dietetics Code of } \\
\text { Ethics. }\end{array}$} & Rarely & -2.3263 & 2.2122 & -6.6621 & 2.0094 & 1.11 & 0.2930 \\
\hline & Sometimes & -1.8645 & 0.8406 & -3.5120 & -0.2170 & 4.92 & 0.0265 \\
\hline & Often & -1.0667 & 0.6905 & -2.4200 & 0.2867 & 2.39 & 0.1224 \\
\hline & Always & 0.0000 & 0.0000 & 0.0000 & 0.0000 & & \\
\hline \multirow{5}{*}{$\begin{array}{l}\text { I use nutrition } \\
\text { information to } \\
\text { diagnose health } \\
\text { conditions in family } \\
\text { members and } \\
\text { individuals. }\end{array}$} & Never & -1.3326 & 1.1705 & -3.6267 & 0.9615 & 1.30 & 0.2549 \\
\hline & Rarely & -1.2410 & 0.8832 & -2.9720 & 0.4900 & 1.97 & 0.1600 \\
\hline & Sometimes & -1.1268 & 0.8904 & -2.8720 & 0.6185 & 1.60 & 0.2057 \\
\hline & Often & -1.0443 & 0.9217 & -2.8508 & 0.7622 & 1.28 & 0.2572 \\
\hline & Always & 0.0000 & 0.0000 & 0.0000 & 0.0000 & & \\
\hline \multirow{2}{*}{ Major } & Dietetics & 0.8858 & 0.5088 & -0.1114 & 1.8830 & 3.03 & 0.0817 \\
\hline & Human Ntr & 0.0000 & 0.0000 & 0.0000 & 0.0000 & & \\
\hline
\end{tabular}


Table 12 (Part B): SOLICITED ADVICE: Make smart food decisions when eating away from home.

\begin{tabular}{|l|r|r|}
\hline \multicolumn{3}{|c|}{ SOLICITED ADVICE: Make smart food decisions when eating away from home. } \\
\hline Source & Chi-Square & Pr > ChiSq \\
\hline $\begin{array}{l}\text { I only deliver information allowed at the student level by the CDR, as } \\
\text { specified in the Dietetic Scope of Practice. }\end{array}$ & 4.66 & 0.1985 \\
\hline $\begin{array}{l}\text { I comprehend the scope of practice. } \\
\text { I refer friends and family to other sources that are outside my scope of } \\
\text { practice. }\end{array}$ & 3.57 & 0.7965 \\
\hline $\begin{array}{l}\text { I abide by the Dietetics Code of Ethics. } \\
\text { I use nutrition information to diagnose health conditions in family } \\
\text { members and individuals. }\end{array}$ & 5.60 & 0.1327 \\
\hline Major & 2.23 & 0.6934 \\
\hline
\end{tabular}


Table 13 (Part A): SOLICITED ADVICE: Use the Internet as a resource for nutrition facts at restaurants.

\begin{tabular}{|c|c|c|c|c|c|c|c|}
\hline \multicolumn{8}{|c|}{ SOLICITED ADVICE: Use the Internet as a resource for nutrition facts at restaurants. } \\
\hline \multirow{5}{*}{\begin{tabular}{|l|} 
Parameter \\
I only deliver \\
information \\
allowed at the \\
student level by the \\
CDR, as specified in \\
the Dietetic Scope \\
of Practice.
\end{tabular}} & \multirow{2}{*}{\begin{tabular}{|l} 
Response \\
Rarely \\
\end{tabular}} & \multirow{2}{*}{\begin{tabular}{r|} 
Estimate \\
0.0153
\end{tabular}} & \multirow{2}{*}{$\begin{array}{l}\begin{array}{l}\text { Standard } \\
\text { Error }\end{array} \\
2.4185\end{array}$} & \multicolumn{2}{|c|}{$\begin{array}{l}\text { Wald 95\% } \\
\text { Confidence Limits }\end{array}$} & \multirow{2}{*}{\begin{tabular}{|r}
$\begin{array}{l}\text { Wald } \\
\text { Chi-Square }\end{array}$ \\
0.00
\end{tabular}} & \multirow{2}{*}{\begin{tabular}{|l|}
$\begin{array}{l}\operatorname{Pr}> \\
\text { ChiSq }\end{array}$ \\
0.995
\end{tabular}} \\
\hline & & & & -4.7249 & 4.7555 & & \\
\hline & Sometimes & 1.1501 & 0.9413 & -0.6948 & 2.9950 & 1.49 & 0.2218 \\
\hline & Often & 0.0881 & 0.9518 & -1.7775 & 1.9537 & 0.01 & 0.9262 \\
\hline & Always & 0.0000 & 0.0000 & 0.0000 & 0.0000 & & \\
\hline \multirow{5}{*}{$\begin{array}{l}\text { I comprehend the } \\
\text { scope of practice. }\end{array}$} & Never & 22.5452 & 76839.27 & -150580 & 150624.7 & 0.00 & 0.9998 \\
\hline & Rarely & -1.2846 & 1.5192 & -4.2622 & 1.6929 & 0.72 & 0.3978 \\
\hline & Sometimes & -0.6354 & 0.8592 & -2.3195 & 1.0486 & 0.55 & 0.4596 \\
\hline & Often & 0.0047 & 0.7855 & -1.5350 & 1.5443 & 0.00 & 0.9953 \\
\hline & Always & 0.0000 & 0.0000 & 0.0000 & 0.0000 & & \\
\hline \multirow{5}{*}{$\begin{array}{l}\text { I refer friends and } \\
\text { family to other } \\
\text { sources that are } \\
\text { outside my scope of } \\
\text { practice. }\end{array}$} & Never & 3.3103 & 1.9818 & -0.5739 & 7.1945 & 2.79 & 0.0948 \\
\hline & Rarely & -1.7274 & 1.6783 & -5.0168 & 1.5619 & 1.06 & 0.3033 \\
\hline & Sometimes & 0.1881 & 0.7050 & -1.1938 & 1.5699 & 0.07 & 0.7897 \\
\hline & Often & 0.4491 & 0.6362 & -0.7978 & 1.6960 & 0.50 & 0.4802 \\
\hline & Always & 0.0000 & 0.0000 & 0.0000 & 0.0000 & & \\
\hline \multirow{4}{*}{$\begin{array}{l}\text { I abide by the } \\
\text { Dietetics Code of } \\
\text { Ethics. }\end{array}$} & Rarely & 0.1001 & 2.3806 & -4.5658 & 4.7661 & 0.00 & 0.9664 \\
\hline & Sometimes & -2.1508 & 0.8390 & -3.7951 & -0.5064 & 6.57 & 0.0104 \\
\hline & Often & -1.6311 & 0.6751 & -2.9543 & -0.3079 & 5.84 & 0.0157 \\
\hline & Always & 0.0000 & 0.0000 & 0.0000 & 0.0000 & & \\
\hline \multirow{5}{*}{$\begin{array}{l}\text { I use nutrition } \\
\text { information to } \\
\text { diagnose health } \\
\text { conditions in family } \\
\text { members and } \\
\text { individuals. }\end{array}$} & Never & -2.8132 & 1.0709 & -4.9121 & -0.7144 & 6.90 & 0.0086 \\
\hline & Rarely & -0.6162 & 0.8042 & -2.1924 & 0.9599 & 0.59 & 0.4435 \\
\hline & Sometimes & -1.4872 & 0.8294 & -3.1127 & 0.1384 & 3.22 & 0.0730 \\
\hline & Often & -1.3733 & 0.8702 & -3.0788 & 0.3322 & 2.49 & 0.1145 \\
\hline & Always & 0.0000 & 0.0000 & 0.0000 & 0.0000 & & \\
\hline \multirow{2}{*}{ Major } & Dietetics & 0.4142 & 0.4989 & -0.5637 & 1.3920 & 0.69 & 0.4064 \\
\hline & Human Ntr & 0.0000 & 0.0000 & 0.0000 & 0.0000 & & \\
\hline
\end{tabular}


Table 13 (Part B): SOLICITED ADVICE: Use the Internet as a resource for nutrition facts at restaurants.

\begin{tabular}{|l|r|r|}
\hline \multicolumn{3}{|c|}{ SOLICITED ADVICE: Use the Internet as a resource for nutrition facts at restaurants. } \\
\hline Source & Chi-Square & Pr > ChiSq \\
\hline $\begin{array}{l}\text { I only deliver information allowed at the student level by the CDR, as } \\
\text { specified in the Dietetic Scope of Practice. }\end{array}$ & 4.10 & 0.2505 \\
\hline I comprehend the scope of practice. & 3.50 & 0.4781 \\
\hline $\begin{array}{l}\text { I refer friends and family to other sources that are outside my scope of } \\
\text { practice. }\end{array}$ & 4.81 & 0.3078 \\
\hline I abide by the Dietetics Code of Ethics. & 7.91 & 0.0479 \\
\hline $\begin{array}{l}\text { I use nutrition information to diagnose health conditions in family } \\
\text { members and individuals. }\end{array}$ & 9.01 & 0.0608 \\
\hline Major & 0.69 & 0.4058 \\
\hline
\end{tabular}


Table 14 (Part A): UNSOLICITED ADVICE: Eat a high protein, low carbohydrate diet.

\begin{tabular}{|c|c|c|c|c|c|c|c|}
\hline \multicolumn{8}{|c|}{ UNSOLICITED ADVICE: Eat a high protein, low carbohydrate diet } \\
\hline \multirow{5}{*}{$\begin{array}{l}\text { Parameter } \\
\text { I only deliver } \\
\text { information } \\
\text { allowed at the } \\
\text { student level by the } \\
\text { CDR, as specified in } \\
\text { the Dietetic Scope } \\
\text { of Practice. }\end{array}$} & \multirow{2}{*}{\begin{tabular}{|l} 
Response \\
Rarely
\end{tabular}} & \multirow{2}{*}{\begin{tabular}{r|} 
Estimate \\
4.1955
\end{tabular}} & \multirow{2}{*}{$\begin{array}{l}\text { Standard } \\
\text { Error } \\
2.5143\end{array}$} & \multicolumn{2}{|c|}{$\begin{array}{l}\text { Wald 95\% } \\
\text { Confidence Limits }\end{array}$} & \multirow{2}{*}{\begin{tabular}{|r|}
$\mid \begin{array}{l}\text { Wald } \\
\text { Chi-Square }\end{array}$ \\
2.78
\end{tabular}} & \multirow{2}{*}{ 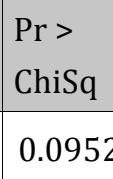 } \\
\hline & & & & -0.7326 & 9.1235 & & \\
\hline & Sometimes & -0.6672 & 1.0709 & -2.7661 & 1.4317 & 0.39 & 0.5333 \\
\hline & Often & -1.2160 & 1.1196 & -3.4103 & 0.9783 & 1.18 & 0.2774 \\
\hline & Always & 0.0000 & 0.0000 & 0.0000 & 0.0000 & & \\
\hline \multirow{5}{*}{$\begin{array}{l}\text { I comprehend the } \\
\text { scope of practice. }\end{array}$} & Never & -23.3898 & 167716.6 & -328742 & 328695.2 & 0.00 & 0.9999 \\
\hline & Rarely & -1.4278 & 1.8617 & -5.0766 & 2.2211 & 0.59 & 0.4431 \\
\hline & Sometimes & 0.9198 & 1.2631 & -1.5559 & 3.3955 & 0.53 & 0.4665 \\
\hline & Often & 1.6038 & 1.1515 & -0.6532 & 3.8607 & 1.94 & 0.1637 \\
\hline & Always & 0.0000 & 0.0000 & 0.0000 & 0.0000 & & \\
\hline \multirow{5}{*}{$\begin{array}{l}\text { I refer friends and } \\
\text { family to other } \\
\text { sources that are } \\
\text { outside my scope } \\
\text { of practice. }\end{array}$} & Never & -21.0576 & 167716.6 & -328740 & 328697.5 & 0.00 & 0.9999 \\
\hline & Rarely & 3.0149 & 1.8927 & -0.6948 & 6.7245 & 2.54 & 0.1112 \\
\hline & Sometimes & 3.0668 & 0.9497 & 1.2054 & 4.9283 & 10.43 & 0.0012 \\
\hline & Often & 1.6221 & 0.8542 & -0.0521 & 3.2964 & 3.61 & 0.0576 \\
\hline & Always & 0.0000 & 0.0000 & 0.0000 & 0.0000 & & \\
\hline \multirow{4}{*}{$\begin{array}{l}\text { I abide by the } \\
\text { Dietetics Code of } \\
\text { Ethics. }\end{array}$} & Rarely & -2.7129 & 2.4245 & -7.4648 & 2.0390 & 1.25 & 0.2632 \\
\hline & Sometimes & -1.5296 & 0.9320 & -3.3564 & 0.2971 & 2.69 & 0.1008 \\
\hline & Often & -0.4169 & 0.7529 & -1.8926 & 1.0587 & 0.31 & 0.5798 \\
\hline & Always & 0.0000 & 0.0000 & 0.0000 & 0.0000 & & \\
\hline \multirow{5}{*}{$\begin{array}{l}\text { I use nutrition } \\
\text { information to } \\
\text { diagnose health } \\
\text { conditions in } \\
\text { family members } \\
\text { and individuals. }\end{array}$} & Never & -3.2455 & 1.4348 & -6.0576 & -0.4334 & 5.12 & 0.0237 \\
\hline & Rarely & -4.0055 & 1.1183 & -6.1972 & -1.8137 & 12.83 & 0.0003 \\
\hline & Sometimes & -1.8974 & 0.9639 & -3.7866 & -0.0082 & 3.87 & 0.0490 \\
\hline & Often & -0.3110 & 0.9890 & -2.2494 & 1.6273 & 0.10 & 0.7531 \\
\hline & Always & 0.0000 & 0.0000 & 0.0000 & 0.0000 & & \\
\hline \multirow{2}{*}{ Major } & Dietetics & 0.2177 & 0.5955 & -0.9494 & 1.3848 & 0.13 & 0.7146 \\
\hline & Human Ntr & 0.0000 & 0.0000 & 0.0000 & 0.0000 & & \\
\hline
\end{tabular}


Table 14 (Part B): UNSOLICITED ADVICE: Eat a high protein, low carbohydrate diet.

\begin{tabular}{|l|r|r|}
\hline \multicolumn{3}{|c|}{ UNSOLICITED ADVICE: Eat a high protein, low carbohydrate diet } \\
\hline Source & Chi-Square & Pr > ChiSq \\
\hline $\begin{array}{l}\text { I only deliver information allowed at the student level by the CDR, as } \\
\text { specified in the Dietetic Scope of Practice. }\end{array}$ & 5.79 & 0.1225 \\
\hline I comprehend the scope of practice. & 7.39 & 0.1166 \\
\hline $\begin{array}{l}\text { I refer friends and family to other sources that are outside my scope of } \\
\text { practice. }\end{array}$ & 13.58 & 0.0088 \\
\hline I abide by the Dietetics Code of Ethics. & 3.78 & 0.2862 \\
\hline $\begin{array}{l}\text { I use nutrition information to diagnose health conditions in family } \\
\text { members and individuals. }\end{array}$ & 29.18 & $<.0001$ \\
\hline Major & 0.13 & 0.7146 \\
\hline
\end{tabular}


Table 15 (Part A): UNSOLICITED ADVICE: Get at least 60 minutes of exercise per day, most days of the week.

\begin{tabular}{|c|c|c|c|c|c|c|c|}
\hline \multicolumn{8}{|c|}{ UNSOLICITED ADVICE: Get at least 60 minutes of exercise per day, most days of the week. } \\
\hline \multirow{5}{*}{$\begin{array}{l}\text { Parameter } \\
\text { I only deliver } \\
\text { information } \\
\text { allowed at the } \\
\text { student level by the } \\
\text { CDR, as specified in } \\
\text { the Dietetic Scope of } \\
\text { Practice. }\end{array}$} & \multirow{2}{*}{\begin{tabular}{|l} 
Response \\
Rarely
\end{tabular}} & \multirow{2}{*}{$\begin{array}{r}\text { Estimate } \\
28.5606\end{array}$} & \multirow{2}{*}{\begin{tabular}{|r}
$\begin{array}{l}\text { Standard } \\
\text { Error }\end{array}$ \\
1.6227
\end{tabular}} & \multicolumn{2}{|c|}{$\begin{array}{l}\text { Wald 95\% } \\
\text { Confidence Limits }\end{array}$} & \multirow{2}{*}{\begin{tabular}{|r}
$\begin{array}{l}\text { Wald } \\
\text { Chi-Square }\end{array}$ \\
309.79
\end{tabular}} & \multirow{2}{*}{\begin{tabular}{|l|}
$\operatorname{Pr}>$ \\
ChiSq \\
$<.000$
\end{tabular}} \\
\hline & & & & 25.3802 & 31.7410 & & \\
\hline & Sometimes & 0.2867 & 0.8929 & -1.4634 & 2.0367 & 0.10 & 0.7482 \\
\hline & Often & 0.3916 & 0.9072 & -1.3865 & 2.1697 & 0.19 & 0.6660 \\
\hline & Always & 0.0000 & 0.0000 & 0.0000 & 0.0000 & & \\
\hline \multirow{5}{*}{$\begin{array}{l}\text { I comprehend the } \\
\text { scope of practice. }\end{array}$} & Never & -27.2096 & 126281.1 & -247534 & 247479.2 & 0.00 & 0.9998 \\
\hline & Rarely & -2.7111 & 1.3259 & -5.3098 & -0.1124 & 4.18 & 0.0409 \\
\hline & Sometimes & -1.1040 & 0.8678 & -2.8048 & 0.5968 & 1.62 & 0.2033 \\
\hline & Often & -1.9763 & 0.8143 & -3.5724 & -0.3803 & 5.89 & 0.0152 \\
\hline & Always & 0.0000 & 0.0000 & 0.0000 & 0.0000 & & \\
\hline \multirow{5}{*}{$\begin{array}{l}\text { I refer friends and } \\
\text { family to other } \\
\text { sources that are } \\
\text { outside my scope of } \\
\text { practice. }\end{array}$} & Never & -24.8563 & 126281.1 & -247531 & 247481.6 & 0.00 & 0.9998 \\
\hline & Rarely & 1.2179 & 1.5169 & -1.7552 & 4.1911 & 0.64 & 0.4220 \\
\hline & Sometimes & -0.0416 & 0.6788 & -1.3721 & 1.2889 & 0.00 & 0.9511 \\
\hline & Often & -0.2267 & 0.5726 & -1.3490 & 0.8955 & 0.16 & 0.6921 \\
\hline & Always & 0.0000 & 0.0000 & 0.0000 & 0.0000 & & \\
\hline \multirow{4}{*}{$\begin{array}{l}\text { I abide by the } \\
\text { Dietetics Code of } \\
\text { Ethics. }\end{array}$} & Rarely & -28.5831 & 0.0000 & -28.5831 & -28.5831 & & \\
\hline & Sometimes & -1.4796 & 0.7878 & -3.0237 & 0.0645 & 3.53 & 0.0604 \\
\hline & Often & -0.7145 & 0.6363 & -1.9617 & 0.5327 & 1.26 & 0.2615 \\
\hline & Always & 0.0000 & 0.0000 & 0.0000 & 0.0000 & & \\
\hline \multirow{5}{*}{$\begin{array}{l}\text { I use nutrition } \\
\text { information to } \\
\text { diagnose health } \\
\text { conditions in family } \\
\text { members and } \\
\text { individuals. }\end{array}$} & Never & 0.1862 & 1.0179 & -1.8089 & 2.1813 & 0.03 & 0.8549 \\
\hline & Rarely & -0.5113 & 0.7978 & -2.0749 & 1.0523 & 0.41 & 0.5216 \\
\hline & Sometimes & 0.1803 & 0.8028 & -1.3931 & 1.7537 & 0.05 & 0.8223 \\
\hline & Often & 1.1941 & 0.8455 & -0.4630 & 2.8512 & 1.99 & 0.1578 \\
\hline & Always & 0.0000 & 0.0000 & 0.0000 & 0.0000 & & \\
\hline \multirow{2}{*}{ Major } & Dietetics & -0.8038 & 0.4874 & -1.7590 & 0.1514 & 2.72 & 0.0991 \\
\hline & Human Ntr & 0.0000 & 0.0000 & 0.0000 & 0.0000 & & \\
\hline
\end{tabular}


Table 15 (Part B): UNSOLICITED ADVICE: Get at least 60 minutes of exercise per day, most days of the week.

UNSOLICITED ADVICE: Get at least 60 minutes of exercise per day, most days of the week.

\begin{tabular}{|l|r|r|}
\hline Source & Chi-Square & $\operatorname{Pr}>$ ChiSq \\
\hline $\begin{array}{l}\text { I only deliver information allowed at the student level by the CDR, as } \\
\text { specified in the Dietetic Scope of Practice. }\end{array}$ & 13.72 & 0.0033 \\
\hline I comprehend the scope of practice. & 13.79 & 0.0080 \\
\hline $\begin{array}{l}\text { I refer friends and family to other sources that are outside my scope of } \\
\text { practice. }\end{array}$ & 3.79 & 0.4352 \\
\hline I abide by the Dietetics Code of Ethics. & 13.21 & 0.0042 \\
\hline $\begin{array}{l}\text { I use nutrition information to diagnose health conditions in family } \\
\text { members and individuals. }\end{array}$ & 7.90 & 0.0953 \\
\hline Major & 2.74 & 0.0978 \\
\hline
\end{tabular}


Table 16 (Part A): UNSOLICITED ADVICE: Eat several smaller meals throughout the day instead of 3 large meals.

\begin{tabular}{|c|c|c|c|c|c|c|c|}
\hline \multirow{5}{*}{$\begin{array}{l}\text { Parameter } \\
\text { I only deliver } \\
\text { information allowed } \\
\text { at the student level } \\
\text { by the CDR, as } \\
\text { specified in the } \\
\text { Dietetic Scope of } \\
\text { Practice. }\end{array}$} & \multirow{2}{*}{$\begin{array}{l}\text { Response } \\
\text { Rarely }\end{array}$} & \multirow{2}{*}{\begin{tabular}{|r} 
Estimate \\
0.7025
\end{tabular}} & \multirow{2}{*}{$\begin{array}{l}\text { Standard } \\
\text { Error } \\
1.9041\end{array}$} & \multicolumn{2}{|c|}{$\begin{array}{l}\text { Wald 95\% } \\
\text { Confidence Limits }\end{array}$} & \multirow{2}{*}{$\begin{array}{l}\text { Wald } \\
\text { Chi-Square } \\
0.14\end{array}$} & \multirow{2}{*}{$\begin{array}{l}\begin{array}{l}\mathrm{Pr}> \\
\text { ChiSq }\end{array} \\
0.7122\end{array}$} \\
\hline & & & & -3.0295 & 4.4345 & & \\
\hline & Sometimes & 1.1061 & 0.8548 & -0.5694 & 2.7815 & 1.67 & 0.1957 \\
\hline & Often & 0.7630 & 0.8607 & -0.9239 & 2.4500 & 0.79 & 0.3753 \\
\hline & Always & 0.0000 & 0.0000 & 0.0000 & 0.0000 & & \\
\hline \multirow{5}{*}{$\begin{array}{l}\text { I comprehend the } \\
\text { scope of practice. }\end{array}$} & Never & 24.4100 & 117783.8 & -230828 & 230876.5 & 0.00 & 0.9998 \\
\hline & Rarely & -1.0642 & 1.1756 & -3.3683 & 1.2399 & 0.82 & 0.3653 \\
\hline & Sometimes & -0.3707 & 0.7970 & -1.9329 & 1.1914 & 0.22 & 0.6418 \\
\hline & Often & -0.7305 & 0.7324 & -2.1660 & 0.7050 & 0.99 & 0.3186 \\
\hline & Always & 0.0000 & 0.0000 & 0.0000 & 0.0000 & & \\
\hline \multirow{5}{*}{$\begin{array}{l}\text { I refer friends and } \\
\text { family to other } \\
\text { sources that are } \\
\text { outside my scope of } \\
\text { practice. }\end{array}$} & Never & -24.6504 & 122602.6 & -240321 & 240272.0 & 0.00 & 0.9998 \\
\hline & Rarely & 0.2994 & 1.5116 & -2.6633 & 3.2621 & 0.04 & 0.8430 \\
\hline & Sometimes & -0.5264 & 0.6586 & -1.8172 & 0.7644 & 0.64 & 0.4241 \\
\hline & Often & -0.9290 & 0.5648 & -2.0360 & 0.1780 & 2.71 & 0.1000 \\
\hline & Always & 0.0000 & 0.0000 & 0.0000 & 0.0000 & & \\
\hline \multirow{4}{*}{$\begin{array}{l}\text { I abide by the } \\
\text { Dietetics Code of } \\
\text { Ethics. }\end{array}$} & Rarely & 2.1694 & 2.0306 & -1.8105 & 6.1493 & 1.14 & 0.2854 \\
\hline & Sometimes & -0.5026 & 0.7139 & -1.9019 & 0.8966 & 0.50 & 0.4814 \\
\hline & Often & -0.9279 & 0.5720 & -2.0490 & 0.1933 & 2.63 & 0.1048 \\
\hline & Always & 0.0000 & 0.0000 & 0.0000 & 0.0000 & & \\
\hline \multirow{5}{*}{$\begin{array}{l}\text { I use nutrition } \\
\text { information to } \\
\text { diagnose health } \\
\text { conditions in family } \\
\text { members and } \\
\text { individuals. }\end{array}$} & Never & 0.0907 & 0.9689 & -1.8082 & 1.9896 & 0.01 & 0.9254 \\
\hline & Rarely & -0.0474 & 0.8218 & -1.6581 & 1.5633 & 0.00 & 0.9540 \\
\hline & Sometimes & 1.2947 & 0.8171 & -0.3067 & 2.8961 & 2.51 & 0.1130 \\
\hline & Often & 1.8025 & 0.9001 & 0.0384 & 3.5666 & 4.01 & 0.0452 \\
\hline & Always & 0.0000 & 0.0000 & 0.0000 & 0.0000 & & \\
\hline \multirow{2}{*}{ Major } & Dietetics & -0.1238 & 0.4805 & -1.0657 & 0.8180 & 0.07 & 0.7967 \\
\hline & Human Ntr & 0.0000 & 0.0000 & 0.0000 & 0.0000 & & \\
\hline
\end{tabular}


Table 16 (Part B): UNSOLICITED ADVICE: Eat several smaller meals throughout the day instead of 3 large meals.

UNSOLICITED ADVICE: Eat several smaller meals throughout the day instead of 3 large meals.

\begin{tabular}{|l|r|r|}
\hline Source & Chi-Square & Pr > ChiSq \\
\hline $\begin{array}{l}\text { I only deliver information allowed at the student level by the CDR, as } \\
\text { specified in the Dietetic Scope of Practice. }\end{array}$ & 1.80 & 0.6155 \\
\hline $\begin{array}{l}\text { I comprehend the scope of practice. } \\
\text { I refer friends and family to other sources that are outside my scope of } \\
\text { practice. }\end{array}$ & 4.65 & 0.3250 \\
\hline I abide by the Dietetics Code of Ethics. & 4.24 & 0.2631 \\
\hline $\begin{array}{l}\text { I use nutrition information to diagnose health conditions in family } \\
\text { members and individuals. }\end{array}$ & 11.78 & 0.2502 \\
\hline Major & 0.07 & 0.7966 \\
\hline
\end{tabular}


Table 17 (Part A): UNSOLICITED ADVICE: Avoid eating or drinking anything after dinner.

\begin{tabular}{|c|c|c|c|c|c|c|c|}
\hline \multicolumn{8}{|c|}{ UNSOLICITED ADVICE: Avoid eating or drinking anything after dinner. } \\
\hline \multirow{5}{*}{$\begin{array}{l}\text { Parameter } \\
\text { I only deliver } \\
\text { information } \\
\text { allowed at the } \\
\text { student level by the } \\
\text { CDR, as specified in } \\
\text { the Dietetic Scope } \\
\text { of Practice. }\end{array}$} & \multirow{2}{*}{$\begin{array}{l}\text { Response } \\
\text { Rarely }\end{array}$} & \multirow{2}{*}{$\begin{array}{r}\text { Estimate } \\
6.0459\end{array}$} & \multirow{2}{*}{$\begin{array}{l}\text { Standard } \\
\text { Error } \\
2.4740\end{array}$} & \multicolumn{2}{|c|}{$\begin{array}{l}\text { Wald } 95 \% \\
\text { Confidence Limits }\end{array}$} & \multirow{2}{*}{\begin{tabular}{|r}
$\begin{array}{l}\text { Wald } \\
\text { Chi-Square }\end{array}$ \\
5.97
\end{tabular}} & \multirow{2}{*}{$\begin{array}{l}\begin{array}{l}\text { Pr }> \\
\text { ChiSq }\end{array} \\
0.0145\end{array}$} \\
\hline & & & & 1.1969 & 10.8948 & & \\
\hline & Sometimes & 0.4978 & 0.9860 & -1.4347 & 2.4303 & 0.25 & 0.6137 \\
\hline & Often & -0.0304 & 1.0090 & -2.0080 & 1.9472 & 0.00 & 0.9760 \\
\hline & Always & 0.0000 & 0.0000 & 0.0000 & 0.0000 & & \\
\hline \multirow{5}{*}{$\begin{array}{l}\text { I comprehend the } \\
\text { scope of practice. }\end{array}$} & Never & -22.7751 & 92035.46 & -180409 & 180363.4 & 0.00 & 0.9998 \\
\hline & Rarely & -0.7868 & 1.6909 & -4.1008 & 2.5273 & 0.22 & 0.6417 \\
\hline & Sometimes & 1.8032 & 0.9763 & -0.1103 & 3.7167 & 3.41 & 0.0647 \\
\hline & Often & 1.4295 & 0.9085 & -0.3512 & 3.2102 & 2.48 & 0.1156 \\
\hline & Always & 0.0000 & 0.0000 & 0.0000 & 0.0000 & & \\
\hline \multirow{5}{*}{$\begin{array}{l}\text { I refer friends and } \\
\text { family to other } \\
\text { sources that are } \\
\text { outside my scope of } \\
\text { practice. }\end{array}$} & Never & -21.6241 & 92035.46 & -180408 & 180364.6 & 0.00 & 0.9998 \\
\hline & Rarely & 1.9348 & 2.1910 & -2.3595 & 6.2291 & 0.78 & 0.3772 \\
\hline & Sometimes & 1.0855 & 0.7480 & -0.3805 & 2.5516 & 2.11 & 0.1467 \\
\hline & Often & 0.8429 & 0.6186 & -0.3696 & 2.0554 & 1.86 & 0.1730 \\
\hline & Always & 0.0000 & 0.0000 & 0.0000 & 0.0000 & & \\
\hline \multirow{4}{*}{$\begin{array}{l}\text { I abide by the } \\
\text { Dietetics Code of } \\
\text { Ethics. }\end{array}$} & Rarely & -6.9396 & 2.4495 & -11.7405 & -2.1388 & 8.03 & 0.0046 \\
\hline & Sometimes & -1.5490 & 0.7847 & -3.0869 & -0.0110 & 3.90 & 0.0484 \\
\hline & Often & -0.7143 & 0.6445 & -1.9774 & 0.5488 & 1.23 & 0.2677 \\
\hline & Always & 0.0000 & 0.0000 & 0.0000 & 0.0000 & & \\
\hline \multirow{5}{*}{$\begin{array}{l}\text { I use nutrition } \\
\text { information to } \\
\text { diagnose health } \\
\text { conditions in family } \\
\text { members and } \\
\text { individuals. }\end{array}$} & Never & -1.9950 & 1.1638 & -4.2760 & 0.2859 & 2.94 & 0.0865 \\
\hline & Rarely & -2.1180 & 0.9135 & -3.9084 & -0.3277 & 5.38 & 0.0204 \\
\hline & Sometimes & -1.4999 & 0.8855 & -3.2355 & 0.2357 & 2.87 & 0.0903 \\
\hline & Often & -0.2685 & 0.8932 & -2.0192 & 1.4821 & 0.09 & 0.7637 \\
\hline & Always & 0.0000 & 0.0000 & 0.0000 & 0.0000 & & \\
\hline \multirow{2}{*}{ Major } & Dietetics & -0.0479 & 0.5162 & -1.0596 & 0.9638 & 0.01 & 0.9260 \\
\hline & Human Ntr & 0.0000 & 0.0000 & 0.0000 & 0.0000 & & \\
\hline
\end{tabular}


Table 17 (Part B): UNSOLICITED ADVICE: Avoid eating or drinking anything after dinner.

\begin{tabular}{|l|r|r|}
\hline \multicolumn{3}{|c|}{ UNSOLICITED ADVICE: Avoid eating or drinking anything after dinner. } \\
\hline Source & Chi-Square & Pr > ChiSq \\
\hline $\begin{array}{l}\text { I only deliver information allowed at the student level by the CDR, as } \\
\text { specified in the Dietetic Scope of Practice. }\end{array}$ & 7.91 & 0.0479 \\
\hline I comprehend the scope of practice. & 8.93 & 0.0629 \\
\hline $\begin{array}{l}\text { I refer friends and family to other sources that are outside my scope of } \\
\text { practice. }\end{array}$ & 3.71 & 0.4462 \\
\hline $\begin{array}{l}\text { I abide by the Dietetics Code of Ethics. } \\
\text { I use nutrition information to diagnose health conditions in family } \\
\text { members and individuals. }\end{array}$ & 13.23 & 0.96 \\
\hline Major & 0.01 & 0.0189 \\
\hline
\end{tabular}


Table 18 (Part A): UNSOLICITED ADVICE: Eat a high protein, high calorie diet.

\begin{tabular}{|c|c|c|c|c|c|c|c|}
\hline \multicolumn{8}{|c|}{ UNSOLICITED ADVICE: Eat a high protein, high calorie diet. } \\
\hline \multirow{5}{*}{$\begin{array}{l}\text { Parameter } \\
\text { I only deliver } \\
\text { information } \\
\text { allowed at the } \\
\text { student level by the } \\
\text { CDR, as specified in } \\
\text { the Dietetic Scope } \\
\text { of Practice. }\end{array}$} & \multirow{2}{*}{$\begin{array}{l}\text { Response } \\
\text { Rarely }\end{array}$} & \multirow{2}{*}{$\begin{array}{r}\text { Estimate } \\
4.8817\end{array}$} & \multirow{2}{*}{ 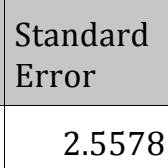 } & \multicolumn{2}{|c|}{$\begin{array}{l}\text { Wald 95\% } \\
\text { Confidence Limits }\end{array}$} & \multirow{2}{*}{\begin{tabular}{|r}
$\begin{array}{l}\text { Wald } \\
\text { Chi-Square }\end{array}$ \\
3.64
\end{tabular}} & \multirow{2}{*}{$\begin{array}{l}\begin{array}{l}\mathrm{Pr}> \\
\text { ChiSq }\end{array} \\
0.0563\end{array}$} \\
\hline & & & & -0.1315 & 9.8948 & & \\
\hline & Sometimes & -0.7923 & 1.0439 & -2.8384 & 1.2537 & 0.58 & 0.4479 \\
\hline & Often & -1.2497 & 1.0546 & -3.3168 & 0.8174 & 1.40 & 0.2360 \\
\hline & Always & 0.0000 & 0.0000 & 0.0000 & 0.0000 & & \\
\hline \multirow{5}{*}{$\begin{array}{l}\text { I comprehend the } \\
\text { scope of practice. }\end{array}$} & Never & -24.7786 & 173677.2 & -340426 & 340376.3 & 0.00 & 0.9999 \\
\hline & Rarely & -2.8506 & 1.8659 & -6.5077 & 0.8066 & 2.33 & 0.1266 \\
\hline & Sometimes & -0.7255 & 1.0753 & -2.8331 & 1.3822 & 0.46 & 0.4999 \\
\hline & Often & 0.0734 & 0.9351 & -1.7593 & 1.9061 & 0.01 & 0.9374 \\
\hline & Always & 0.0000 & 0.0000 & 0.0000 & 0.0000 & & \\
\hline \multirow{5}{*}{$\begin{array}{l}\text { I refer friends and } \\
\text { family to other } \\
\text { sources that are } \\
\text { outside my scope of } \\
\text { practice. }\end{array}$} & Never & -20.5913 & 173677.2 & -340422 & 340380.5 & 0.00 & 0.9999 \\
\hline & Rarely & 5.1200 & 1.9937 & 1.2124 & 9.0275 & 6.60 & 0.0102 \\
\hline & Sometimes & 2.6745 & 0.9231 & 0.8652 & 4.4838 & 8.39 & 0.0038 \\
\hline & Often & 1.4770 & 0.8230 & -0.1359 & 3.0900 & 3.22 & 0.0727 \\
\hline & Always & 0.0000 & 0.0000 & 0.0000 & 0.0000 & & \\
\hline \multirow{4}{*}{$\begin{array}{l}\text { I abide by the } \\
\text { Dietetics Code of } \\
\text { Ethics. }\end{array}$} & Rarely & -3.6796 & 2.3653 & -8.3155 & 0.9564 & 2.42 & 0.1198 \\
\hline & Sometimes & -2.2327 & 0.9571 & -4.1085 & -0.3569 & 5.44 & 0.0197 \\
\hline & Often & -0.1958 & 0.7475 & -1.6609 & 1.2694 & 0.07 & 0.7934 \\
\hline & Always & 0.0000 & 0.0000 & 0.0000 & 0.0000 & & \\
\hline \multirow{5}{*}{$\begin{array}{l}\text { I use nutrition } \\
\text { information to } \\
\text { diagnose health } \\
\text { conditions in family } \\
\text { members and } \\
\text { individuals. }\end{array}$} & Never & -3.0769 & 1.4209 & -5.8619 & -0.2920 & 4.69 & 0.0304 \\
\hline & Rarely & -3.2774 & 1.0564 & -5.3479 & -1.2069 & 9.63 & 0.0019 \\
\hline & Sometimes & -0.9465 & 0.8629 & -2.6377 & 0.7448 & 1.20 & 0.2727 \\
\hline & Often & -0.0409 & 0.9407 & -1.8846 & 1.8029 & 0.00 & 0.9654 \\
\hline & Always & 0.0000 & 0.0000 & 0.0000 & 0.0000 & & \\
\hline \multirow{2}{*}{ Major } & Dietetics & -0.8177 & 0.6145 & -2.0220 & 0.3866 & 1.77 & 0.1833 \\
\hline & Human Ntr & 0.0000 & 0.0000 & 0.0000 & 0.0000 & & \\
\hline
\end{tabular}


Table 18 (Part B): UNSOLICITED ADVICE: Eat a high protein, high calorie diet.

\begin{tabular}{|l|r|r|}
\hline \multicolumn{3}{|c|}{ UNSOLICITED ADVICE: Eat a high protein, high calorie diet. } \\
\hline Source & Chi-Square & $\operatorname{Pr}>$ ChiSq \\
\hline $\begin{array}{l}\text { I only deliver information allowed at the student level by the CDR, as } \\
\text { specified in the Dietetic Scope of Practice. }\end{array}$ & 6.96 & 0.0733 \\
\hline I comprehend the scope of practice. & 5.81 & 0.2139 \\
\hline $\begin{array}{l}\text { I refer friends and family to other sources that are outside my scope of } \\
\text { practice. }\end{array}$ & 13.04 & 0.0111 \\
\hline $\begin{array}{l}\text { I abide by the Dietetics Code of Ethics. } \\
\text { I use nutrition information to diagnose health conditions in family } \\
\text { members and individuals. }\end{array}$ & 9.61 & 0.0222 \\
\hline Major & 22.28 & 0.0002 \\
\hline
\end{tabular}


Table 19 (Part A): UNSOLICITED ADVICE: Avoid calorically dense foods and beverages.

\begin{tabular}{|c|c|c|c|c|c|c|c|}
\hline \multicolumn{8}{|c|}{ UNSOLICITED ADVICE: Avoid calorically dense foods and beverages. } \\
\hline \multirow{5}{*}{$\begin{array}{l}\text { Parameter } \\
\text { I only deliver } \\
\text { information } \\
\text { allowed at the } \\
\text { student level by the } \\
\text { CDR, as specified in } \\
\text { the Dietetic Scope } \\
\text { of Practice. }\end{array}$} & \multirow{2}{*}{$\begin{array}{l}\text { Response } \\
\text { Rarely }\end{array}$} & \multirow{2}{*}{$\begin{array}{r}\text { Estimate } \\
2.3246\end{array}$} & \multirow{2}{*}{$\begin{array}{l}\begin{array}{l}\text { Standard } \\
\text { Error }\end{array} \\
1.8894\end{array}$} & \multicolumn{2}{|c|}{$\begin{array}{l}\text { Wald 95\% } \\
\text { Confidence Limits }\end{array}$} & \multirow{2}{*}{$\begin{array}{l}\text { Wald } \\
\text { Chi-Square } \\
\qquad 1.51\end{array}$} & \multirow{2}{*}{ 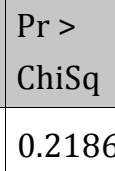 } \\
\hline & & & & -1.3785 & 6.0278 & & \\
\hline & Sometimes & 1.1505 & 0.9335 & -0.6791 & 2.9800 & 1.52 & 0.2178 \\
\hline & Often & 0.6644 & 0.9280 & -1.1545 & 2.4832 & 0.51 & 0.4740 \\
\hline & Always & 0.0000 & 0.0000 & 0.0000 & 0.0000 & & \\
\hline \multirow{5}{*}{$\begin{array}{l}\text { I comprehend the } \\
\text { scope of practice. }\end{array}$} & Never & 0.3770 & 1.8491 & -3.2471 & 4.0011 & 0.04 & 0.8384 \\
\hline & Rarely & 0.7642 & 1.2233 & -1.6334 & 3.1618 & 0.39 & 0.5322 \\
\hline & Sometimes & -0.6525 & 0.7745 & -2.1704 & 0.8654 & 0.71 & 0.3995 \\
\hline & Often & -0.5558 & 0.7096 & -1.9466 & 0.8349 & 0.61 & 0.4334 \\
\hline & Always & 0.0000 & 0.0000 & 0.0000 & 0.0000 & & \\
\hline \multirow{5}{*}{$\begin{array}{l}\text { I refer friends and } \\
\text { family to other } \\
\text { sources that are } \\
\text { outside my scope of } \\
\text { practice. }\end{array}$} & Never & -22.8254 & 78717.28 & -154306 & 154260.2 & 0.00 & 0.9998 \\
\hline & Rarely & 1.2146 & 1.5585 & -1.8399 & 4.2692 & 0.61 & 0.4358 \\
\hline & Sometimes & -0.1516 & 0.6988 & -1.5213 & 1.2181 & 0.05 & 0.8283 \\
\hline & Often & -0.3798 & 0.6126 & -1.5804 & 0.8208 & 0.38 & 0.5352 \\
\hline & Always & 0.0000 & 0.0000 & 0.0000 & 0.0000 & & \\
\hline \multirow{4}{*}{$\begin{array}{l}\text { I abide by the } \\
\text { Dietetics Code of } \\
\text { Ethics. }\end{array}$} & Rarely & -0.7710 & 2.0986 & -4.8841 & 3.3421 & 0.13 & 0.7133 \\
\hline & Sometimes & -1.3272 & 0.7331 & -2.7640 & 0.1095 & 3.28 & 0.0702 \\
\hline & Often & -0.8236 & 0.5959 & -1.9915 & 0.3442 & 1.91 & 0.1669 \\
\hline & Always & 0.0000 & 0.0000 & 0.0000 & 0.0000 & & \\
\hline \multirow{5}{*}{$\begin{array}{l}\text { I use nutrition } \\
\text { information to } \\
\text { diagnose health } \\
\text { conditions in family } \\
\text { members and } \\
\text { individuals. }\end{array}$} & Never & -2.5483 & 1.1281 & -4.7593 & -0.3374 & 5.10 & 0.0239 \\
\hline & Rarely & -2.2594 & 0.9476 & -4.1166 & -0.4023 & 5.69 & 0.0171 \\
\hline & Sometimes & -1.2906 & 0.9122 & -3.0785 & 0.4972 & 2.00 & 0.1571 \\
\hline & Often & -0.7989 & 0.9459 & -2.6528 & 1.0549 & 0.71 & 0.3983 \\
\hline & Always & 0.0000 & 0.0000 & 0.0000 & 0.0000 & & \\
\hline \multirow{2}{*}{ Major } & Dietetics & -0.2633 & 0.4903 & -1.2243 & 0.6977 & 0.29 & 0.5913 \\
\hline & Human Ntr & 0.0000 & 0.0000 & 0.0000 & 0.0000 & & \\
\hline
\end{tabular}


Table 19 (Part B): UNSOLICITED ADVICE: Avoid calorically dense foods and beverages.

\begin{tabular}{|l|r|r|}
\hline \multicolumn{3}{|c|}{ UNSOLICITED ADVICE: Avoid calorically dense foods and beverages. } \\
\hline Source & Chi-Square & Pr > ChiSq \\
\hline $\begin{array}{l}\text { I only deliver information allowed at the student level by the CDR, as } \\
\text { specified in the Dietetic Scope of Practice. }\end{array}$ & 2.55 & 0.4664 \\
\hline I comprehend the scope of practice. & 2.65 & 0.6180 \\
\hline $\begin{array}{l}\text { I refer friends and family to other sources that are outside my scope of } \\
\text { practice. }\end{array}$ & 2.78 & 0.5954 \\
\hline $\begin{array}{l}\text { I abide by the Dietetics Code of Ethics. } \\
\text { I use nutrition information to diagnose health conditions in family } \\
\text { members and individuals. }\end{array}$ & 3.41 & 0.3322 \\
\hline Major & 0.29 & 0.5910 \\
\hline
\end{tabular}


Table 20 (Part A): UNSOLICITED ADVICE: Consume less alcoholic beverages.

\begin{tabular}{|c|c|c|c|c|c|c|c|}
\hline \multicolumn{8}{|c|}{ UNSOLICITED ADVICE: Consume less alcoholic beverages. } \\
\hline \multirow{5}{*}{$\begin{array}{l}\text { Parameter } \\
\text { I only deliver } \\
\text { information } \\
\text { allowed at the } \\
\text { student level by the } \\
\text { CDR, as specified in } \\
\text { the Dietetic Scope of } \\
\text { Practice. }\end{array}$} & \multirow{2}{*}{$\begin{array}{l}\text { Response } \\
\text { Rarely }\end{array}$} & \multirow{2}{*}{$\begin{array}{r}\text { Estimate } \\
3.1513\end{array}$} & \multirow{2}{*}{$\begin{array}{l}\text { Standard } \\
\text { Error } \\
1.9986\end{array}$} & \multicolumn{2}{|c|}{$\begin{array}{l}\text { Wald 95\% } \\
\text { Confidence Limits }\end{array}$} & \multirow{2}{*}{$\begin{array}{l}\text { Wald } \\
\text { Chi-Square } \\
2.49\end{array}$} & \multirow{2}{*}{\begin{tabular}{|l|}
$\begin{array}{l}\text { Pr }> \\
\text { ChiSq }\end{array}$ \\
0.1148
\end{tabular}} \\
\hline & & & & -0.7658 & 7.0685 & & \\
\hline & Sometimes & 1.1801 & 0.8837 & -0.5519 & 2.9121 & 1.78 & 0.1817 \\
\hline & Often & 1.0034 & 0.9091 & -0.7785 & 2.7853 & 1.22 & 0.2697 \\
\hline & Always & 0.0000 & 0.0000 & 0.0000 & 0.0000 & & \\
\hline \multirow{5}{*}{$\begin{array}{l}\text { I comprehend the } \\
\text { scope of practice. }\end{array}$} & Never & 0.9710 & 1.8565 & -2.6676 & 4.6096 & 0.27 & 0.6009 \\
\hline & Rarely & 0.9502 & 1.3256 & -1.6480 & 3.5483 & 0.51 & 0.4735 \\
\hline & Sometimes & 0.2997 & 0.7977 & -1.2638 & 1.8633 & 0.14 & 0.7071 \\
\hline & Often & 0.5358 & 0.7592 & -0.9522 & 2.0237 & 0.50 & 0.4804 \\
\hline & Always & 0.0000 & 0.0000 & 0.0000 & 0.0000 & & \\
\hline \multirow{5}{*}{$\begin{array}{l}\text { I refer friends and } \\
\text { family to other } \\
\text { sources that are } \\
\text { outside my scope of } \\
\text { practice. }\end{array}$} & Never & -24.0659 & 82400.87 & -161527 & 161478.7 & 0.00 & 0.9998 \\
\hline & Rarely & -1.2213 & 1.6924 & -4.5383 & 2.0958 & 0.52 & 0.4705 \\
\hline & Sometimes & 0.2833 & 0.7023 & -1.0932 & 1.6599 & 0.16 & 0.6866 \\
\hline & Often & -0.3599 & 0.5987 & -1.5334 & 0.8136 & 0.36 & 0.5478 \\
\hline & Always & 0.0000 & 0.0000 & 0.0000 & 0.0000 & & \\
\hline \multirow{4}{*}{$\begin{array}{l}\text { I abide by the } \\
\text { Dietetics Code of } \\
\text { Ethics. }\end{array}$} & Rarely & -5.1889 & 2.1361 & -9.3755 & -1.0023 & 5.90 & 0.0151 \\
\hline & Sometimes & -2.8553 & 0.7647 & -4.3541 & -1.3565 & 13.94 & 0.0002 \\
\hline & Often & -1.7260 & 0.6081 & -2.9179 & -0.5341 & 8.06 & 0.0045 \\
\hline & Always & 0.0000 & 0.0000 & 0.0000 & 0.0000 & & \\
\hline \multirow{5}{*}{$\begin{array}{l}\text { I use nutrition } \\
\text { information to } \\
\text { diagnose health } \\
\text { conditions in family } \\
\text { members and } \\
\text { individuals. }\end{array}$} & Never & -0.5183 & 1.0259 & -2.5290 & 1.4925 & 0.26 & 0.6134 \\
\hline & Rarely & -1.7499 & 0.9131 & -3.5395 & 0.0397 & 3.67 & 0.0553 \\
\hline & Sometimes & -0.2128 & 0.8794 & -1.9364 & 1.5108 & 0.06 & 0.8088 \\
\hline & Often & 0.4497 & 0.9184 & -1.3504 & 2.2499 & 0.24 & 0.6244 \\
\hline & Always & 0.0000 & 0.0000 & 0.0000 & 0.0000 & & \\
\hline \multirow{2}{*}{ Major } & Dietetics & -0.2000 & 0.5042 & -1.1882 & 0.7882 & 0.16 & 0.6916 \\
\hline & Human Ntr & 0.0000 & 0.0000 & 0.0000 & 0.0000 & & \\
\hline
\end{tabular}


Table 20 (Part B): UNSOLICITED ADVICE: Consume less alcoholic beverages.

\begin{tabular}{|l|r|r|}
\hline \multicolumn{3}{|c|}{ UNSOLICITED ADVICE: Consume less alcoholic beverages. } \\
\hline Source & Chi-Square & Pr > ChiSq \\
\hline $\begin{array}{l}\text { I only deliver information allowed at the student level by the CDR, as } \\
\text { specified in the Dietetic Scope of Practice. }\end{array}$ & 3.12 & 0.3733 \\
\hline I comprehend the scope of practice. & 0.95 & 0.9174 \\
\hline $\begin{array}{l}\text { I refer friends and family to other sources that are outside my scope of } \\
\text { practice. }\end{array}$ & 4.25 & 0.3729 \\
\hline $\begin{array}{l}\text { I abide by the Dietetics Code of Ethics. } \\
\text { I use nutrition information to diagnose health conditions in family } \\
\text { members and individuals. }\end{array}$ & 17.10 & 0.0007 \\
\hline Major & 0.16 & 0.6918 \\
\hline
\end{tabular}


Table 21 (Part A): UNSOLICITED ADVICE: Make smart food decisions when eating away from home.

\begin{tabular}{|c|c|c|c|c|c|c|c|}
\hline \multicolumn{8}{|c|}{ UNSOLICITED ADVICE: Make smart food decisions when eating away from home. } \\
\hline \multirow{5}{*}{$\begin{array}{l}\text { Parameter } \\
\text { I only deliver } \\
\text { information } \\
\text { allowed at the } \\
\text { student level by the } \\
\text { CDR, as specified in } \\
\text { the Dietetic Scope } \\
\text { of Practice. }\end{array}$} & \multirow{2}{*}{\begin{tabular}{|l|} 
Response \\
Rarely
\end{tabular}} & \multirow{2}{*}{\begin{tabular}{|r|} 
Estimate \\
26.6147
\end{tabular}} & \multirow{2}{*}{\begin{tabular}{|r|}
$\begin{array}{l}\text { Standard } \\
\text { Error }\end{array}$ \\
1.5659
\end{tabular}} & \multicolumn{2}{|c|}{$\begin{array}{l}\text { Wald } 95 \% \\
\text { Confidence Limits }\end{array}$} & \multirow{2}{*}{\begin{tabular}{|r|}
$\begin{array}{l}\text { Wald } \\
\text { Chi-Square }\end{array}$ \\
288.88
\end{tabular}} & \multirow{2}{*}{$\begin{array}{l}\text { Pr }> \\
\text { ChiSq } \\
<.0001\end{array}$} \\
\hline & & & & 23.5456 & 29.6838 & & \\
\hline & Sometimes & 1.0437 & 0.9195 & -0.7585 & 2.8458 & 1.29 & 0.2563 \\
\hline & Often & 0.7570 & 0.9462 & -1.0976 & 2.6115 & 0.64 & 0.4237 \\
\hline & Always & 0.0000 & 0.0000 & 0.0000 & 0.0000 & & \\
\hline \multirow{5}{*}{$\begin{array}{l}\text { I comprehend the } \\
\text { scope of practice. }\end{array}$} & Never & -3.1024 & 1.7570 & -6.5460 & 0.3412 & 3.12 & 0.0774 \\
\hline & Rarely & -0.1969 & 1.2221 & -2.5923 & 2.1984 & 0.03 & 0.8720 \\
\hline & Sometimes & -0.4924 & 0.8742 & -2.2058 & 1.2210 & 0.32 & 0.5732 \\
\hline & Often & -1.2404 & 0.8116 & -2.8312 & 0.3503 & 2.34 & 0.1264 \\
\hline & Always & 0.0000 & 0.0000 & 0.0000 & 0.0000 & & \\
\hline \multirow{5}{*}{$\begin{array}{l}\text { I refer friends and } \\
\text { family to other } \\
\text { sources that are } \\
\text { outside my scope of } \\
\text { practice. }\end{array}$} & Never & -24.2086 & 126952.8 & -248847 & 248798.7 & 0.00 & 0.9998 \\
\hline & Rarely & 0.1438 & 1.4740 & -2.7452 & 3.0328 & 0.01 & 0.9223 \\
\hline & Sometimes & 0.0339 & 0.6932 & -1.3248 & 1.3926 & 0.00 & 0.9610 \\
\hline & Often & -0.2113 & 0.6020 & -1.3912 & 0.9687 & 0.12 & 0.7256 \\
\hline & Always & 0.0000 & 0.0000 & 0.0000 & 0.0000 & & \\
\hline \multirow{4}{*}{$\begin{array}{l}\text { I abide by the } \\
\text { Dietetics Code of } \\
\text { Ethics. }\end{array}$} & Rarely & -25.7858 & 0.0000 & -25.7858 & -25.7858 & & \\
\hline & Sometimes & -2.0112 & 0.8388 & -3.6553 & -0.3671 & 5.75 & 0.0165 \\
\hline & Often & -1.3120 & 0.6903 & -2.6649 & 0.0409 & 3.61 & 0.0573 \\
\hline & Always & 0.0000 & 0.0000 & 0.0000 & 0.0000 & & \\
\hline \multirow{5}{*}{$\begin{array}{l}\text { I use nutrition } \\
\text { information to } \\
\text { diagnose health } \\
\text { conditions in family } \\
\text { members and } \\
\text { individuals. }\end{array}$} & Never & 0.0975 & 1.0314 & -1.9239 & 2.1190 & 0.01 & 0.9247 \\
\hline & Rarely & -0.5395 & 0.7960 & -2.0996 & 1.0206 & 0.46 & 0.4979 \\
\hline & Sometimes & 1.0429 & 0.8460 & -0.6153 & 2.7011 & 1.52 & 0.2177 \\
\hline & Often & 1.3224 & 0.8864 & -0.4148 & 3.0597 & 2.23 & 0.1357 \\
\hline & Always & 0.0000 & 0.0000 & 0.0000 & 0.0000 & & \\
\hline \multirow{2}{*}{ Major } & Dietetics & -0.1777 & 0.4787 & -1.1159 & 0.7605 & 0.14 & 0.7104 \\
\hline & Human Ntr & 0.0000 & 0.0000 & 0.0000 & 0.0000 & & \\
\hline
\end{tabular}


Table 21 (Part B): UNSOLICITED ADVICE: Make smart food decisions when eating away from home.

\begin{tabular}{|l|r|r|}
\hline \multicolumn{3}{|c|}{ UNSOLICITED ADVICE: Make smart food decisions when eating away from home. } \\
\hline Source & Chi-Square & Pr > ChiSq \\
\hline $\begin{array}{l}\text { I only deliver information allowed at the student level by the CDR, as } \\
\text { specified in the Dietetic Scope of Practice. }\end{array}$ & 7.13 & 0.0677 \\
\hline I comprehend the scope of practice. & 6.07 & 0.1940 \\
\hline $\begin{array}{l}\text { I refer friends and family to other sources that are outside my scope of } \\
\text { practice. }\end{array}$ & 2.17 & 0.7048 \\
\hline I abide by the Dietetics Code of Ethics. & 8.81 & 0.0319 \\
\hline $\begin{array}{l}\text { I use nutrition information to diagnose health conditions in family } \\
\text { members and individuals. }\end{array}$ & 10.51 & 0.0327 \\
\hline Major & 0.14 & 0.7103 \\
\hline
\end{tabular}


Table 22 (Part A): UNSOLICITED ADVICE: Use the Internet as a resource for nutrition facts at restaurants.

\begin{tabular}{|c|c|c|c|c|c|c|c|}
\hline \multicolumn{8}{|c|}{ UNSOLICITED ADVICE: Use the Internet as a resource for nutrition facts at restaurants. } \\
\hline \multirow{5}{*}{$\begin{array}{l}\text { Parameter } \\
\text { I only deliver } \\
\text { information allowed } \\
\text { at the student level } \\
\text { by the CDR, as } \\
\text { specified in the } \\
\text { Dietetic Scope of } \\
\text { Practice. }\end{array}$} & \multirow{2}{*}{$\begin{array}{l}\text { Response } \\
\text { Rarely }\end{array}$} & \multirow{2}{*}{\begin{tabular}{r|} 
Estimate \\
3.8936
\end{tabular}} & \multirow{2}{*}{$\begin{array}{l}\text { Standard } \\
\text { Error } \\
2.1150\end{array}$} & \multicolumn{2}{|c|}{$\begin{array}{l}\text { Wald 95\% } \\
\text { Confidence Limits }\end{array}$} & \multirow{2}{*}{$\begin{array}{r}\text { Wald } \\
\text { Chi-Square } \\
3.39\end{array}$} & \multirow{2}{*}{ 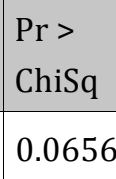 } \\
\hline & & & & -0.2516 & 8.0389 & & \\
\hline & Sometimes & 1.4839 & 0.8545 & -0.1909 & 3.1586 & 3.02 & 0.0825 \\
\hline & Often & 0.7138 & 0.8784 & -1.0079 & 2.4354 & 0.66 & 0.4165 \\
\hline & Always & 0.0000 & 0.0000 & 0.0000 & 0.0000 & & \\
\hline \multirow{5}{*}{$\begin{array}{l}\text { I comprehend the } \\
\text { scope of practice. }\end{array}$} & Never & -1.5184 & 1.7144 & -4.8785 & 1.8417 & 0.78 & 0.3758 \\
\hline & Rarely & -2.7175 & 1.3670 & -5.3967 & -0.0383 & 3.95 & 0.0468 \\
\hline & Sometimes & -2.1834 & 0.8502 & -3.8498 & -0.5171 & 6.60 & 0.0102 \\
\hline & Often & -1.9087 & 0.7791 & -3.4358 & -0.3816 & 6.00 & 0.0143 \\
\hline & Always & 0.0000 & 0.0000 & 0.0000 & 0.0000 & & \\
\hline \multirow{5}{*}{$\begin{array}{l}\text { I refer friends and } \\
\text { family to other } \\
\text { sources that are } \\
\text { outside my scope of } \\
\text { practice. }\end{array}$} & Never & -24.0746 & 131099.8 & -256975 & 256926.8 & 0.00 & 0.9999 \\
\hline & Rarely & -23.9378 & 131099.8 & -256975 & 256927.0 & 0.00 & 0.9999 \\
\hline & Sometimes & -0.2774 & 0.6771 & -1.6044 & 1.0496 & 0.17 & 0.6820 \\
\hline & Often & -0.2448 & 0.5872 & -1.3957 & 0.9060 & 0.17 & 0.6767 \\
\hline & Always & 0.0000 & 0.0000 & 0.0000 & 0.0000 & & \\
\hline \multirow{4}{*}{$\begin{array}{l}\text { I abide by the } \\
\text { Dietetics Code of } \\
\text { Ethics. }\end{array}$} & Rarely & -2.0896 & 2.1475 & -6.2985 & 2.1193 & 0.95 & 0.3305 \\
\hline & Sometimes & -0.9887 & 0.7559 & -2.4702 & 0.4928 & 1.71 & 0.1909 \\
\hline & Often & -0.7322 & 0.6103 & -1.9283 & 0.4638 & 1.44 & 0.2302 \\
\hline & Always & 0.0000 & 0.0000 & 0.0000 & 0.0000 & & \\
\hline \multirow{5}{*}{$\begin{array}{l}\text { I use nutrition } \\
\text { information to } \\
\text { diagnose health } \\
\text { conditions in family } \\
\text { members and } \\
\text { individuals. }\end{array}$} & Never & -0.1812 & 1.0603 & -2.2593 & 1.8969 & 0.03 & 0.8643 \\
\hline & Rarely & 0.0388 & 0.8105 & -1.5498 & 1.6274 & 0.00 & 0.9618 \\
\hline & Sometimes & 0.6707 & 0.8610 & -1.0169 & 2.3583 & 0.61 & 0.4360 \\
\hline & Often & 0.7473 & 0.9018 & -1.0202 & 2.5147 & 0.69 & 0.4073 \\
\hline & Always & 0.0000 & 0.0000 & 0.0000 & 0.0000 & & \\
\hline \multirow{2}{*}{ Major } & Dietetics & -0.1234 & 0.4900 & -1.0838 & 0.8369 & 0.06 & 0.8011 \\
\hline & Human Ntr & 0.0000 & 0.0000 & 0.0000 & 0.0000 & & \\
\hline
\end{tabular}


Table 22 (Part B): UNSOLICITED ADVICE: Use the Internet as a resource for nutrition facts at restaurants.

UNSOLICITED ADVICE: Use the Internet as a resource for nutrition facts at restaurants.

\begin{tabular}{|l|r|r|}
\hline Source & Chi-Square & $\operatorname{Pr}>$ ChiSq \\
\hline $\begin{array}{l}\text { I only deliver information allowed at the student level by the CDR, as } \\
\text { specified in the Dietetic Scope of Practice. }\end{array}$ & 6.06 & 0.1086 \\
\hline I comprehend the scope of practice. & 7.95 & 0.0935 \\
\hline $\begin{array}{l}\text { I refer friends and family to other sources that are outside my scope of } \\
\text { practice. }\end{array}$ & 2.85 & 0.5825 \\
\hline I abide by the Dietetics Code of Ethics. & 2.60 & 0.4576 \\
\hline $\begin{array}{l}\text { I use nutrition information to diagnose health conditions in family } \\
\text { members and individuals. }\end{array}$ & 2.27 & 0.6859 \\
\hline Major & 0.06 & 0.8011 \\
\hline
\end{tabular}


Table 23 (Part A): OVERWEIGHT/OBESE: Eat a high protein, low carbohydrate diet.

\begin{tabular}{|c|c|c|c|c|c|c|c|}
\hline \multicolumn{8}{|c|}{ OVERWEIGHT/OBESE: Eat a high protein, low carbohydrate diet. } \\
\hline \multirow{5}{*}{$\begin{array}{l}\text { Parameter } \\
\text { I only deliver } \\
\text { information } \\
\text { allowed at the } \\
\text { student level by } \\
\text { the CDR, as } \\
\text { specified in the } \\
\text { Dietetic Scope of } \\
\text { Practice. }\end{array}$} & \multirow{2}{*}{$\begin{array}{l}\text { Response } \\
\text { Rarely }\end{array}$} & \multirow{2}{*}{$\begin{array}{l}\text { Estimate } \\
-0.3840\end{array}$} & \multirow{2}{*}{\begin{tabular}{|r|}
$\begin{array}{l}\text { Standard } \\
\text { Error }\end{array}$ \\
2.0443
\end{tabular}} & \multicolumn{2}{|c|}{$\begin{array}{l}\text { Wald 95\% } \\
\text { Confidence Limits }\end{array}$} & \multirow{2}{*}{\begin{tabular}{|r|}
$\begin{array}{l}\text { Wald } \\
\text { Chi-Square }\end{array}$ \\
0.04
\end{tabular}} & \multirow{2}{*}{$\begin{array}{l}\mathrm{Pr}> \\
\text { ChiSq } \\
0.8510\end{array}$} \\
\hline & & & & -4.3908 & 3.6228 & & \\
\hline & Sometimes & -1.1586 & 0.8166 & -2.7590 & 0.4419 & 2.01 & 0.1559 \\
\hline & Often & 0.1312 & 0.8408 & -1.5168 & 1.7792 & 0.02 & 0.8760 \\
\hline & Always & 0.0000 & 0.0000 & 0.0000 & 0.0000 & & \\
\hline \multirow{5}{*}{$\begin{array}{l}\text { I comprehend the } \\
\text { scope of practice. }\end{array}$} & Never & -24.0141 & 87760.45 & -172031 & 171983.3 & 0.00 & 0.9998 \\
\hline & Rarely & 0.9717 & 1.4658 & -1.9012 & 3.8446 & 0.44 & 0.5074 \\
\hline & Sometimes & 0.2758 & 0.8091 & -1.3101 & 1.8617 & 0.12 & 0.7332 \\
\hline & Often & 0.3785 & 0.7314 & -1.0550 & 1.8119 & 0.27 & 0.6048 \\
\hline & Always & 0.0000 & 0.0000 & 0.0000 & 0.0000 & & \\
\hline \multirow{5}{*}{$\begin{array}{l}\text { I refer friends and } \\
\text { family to other } \\
\text { sources that are } \\
\text { outside my scope } \\
\text { of practice. }\end{array}$} & Never & 0.9796 & 1.8072 & -2.5624 & 4.5217 & 0.29 & 0.5878 \\
\hline & Rarely & -0.3200 & 1.6411 & -3.5365 & 2.8966 & 0.04 & 0.8454 \\
\hline & Sometimes & 1.3098 & 0.7076 & -0.0772 & 2.6967 & 3.43 & 0.0642 \\
\hline & Often & 1.1485 & 0.6125 & -0.0519 & 2.3488 & 3.52 & 0.0608 \\
\hline & Always & 0.0000 & 0.0000 & 0.0000 & 0.0000 & & \\
\hline \multirow{4}{*}{$\begin{array}{l}\text { I abide by the } \\
\text { Dietetics Code of } \\
\text { Ethics. }\end{array}$} & Rarely & 0.5118 & 2.1541 & -3.7101 & 4.7337 & 0.06 & 0.8122 \\
\hline & Sometimes & -1.4399 & 0.7729 & -2.9548 & 0.0750 & 3.47 & 0.0625 \\
\hline & Often & -1.2715 & 0.6055 & -2.4583 & -0.0848 & 4.41 & 0.0357 \\
\hline & Always & 0.0000 & 0.0000 & 0.0000 & 0.0000 & & \\
\hline \multirow{5}{*}{$\begin{array}{l}\text { I use nutrition } \\
\text { information to } \\
\text { diagnose health } \\
\text { conditions in } \\
\text { family members } \\
\text { and individuals. }\end{array}$} & Never & -1.8753 & 1.0910 & -4.0136 & 0.2630 & 2.95 & 0.0856 \\
\hline & Rarely & -2.3609 & 0.8803 & -4.0862 & -0.6356 & 7.19 & 0.0073 \\
\hline & Sometimes & -0.9296 & 0.8325 & -2.5612 & 0.7020 & 1.25 & 0.2641 \\
\hline & Often & -0.0568 & 0.9176 & -1.8553 & 1.7417 & 0.00 & 0.9507 \\
\hline & Always & 0.0000 & 0.0000 & 0.0000 & 0.0000 & & \\
\hline \multirow{2}{*}{ Major } & Dietetics & -0.2120 & 0.4976 & -1.1873 & 0.7633 & 0.18 & 0.6701 \\
\hline & Human Ntr & 0.0000 & 0.0000 & 0.0000 & 0.0000 & & \\
\hline
\end{tabular}


Table 23 (Part B): OVERWEIGHT/OBESE: Eat a high protein, low carbohydrate diet.

\begin{tabular}{|l|r|r|}
\hline \multicolumn{3}{|c|}{ OVERWEIGHT/OBESE: Eat a high protein, low carbohydrate diet. } \\
\hline Source & Chi-Square & $\operatorname{Pr}>$ ChiSq \\
\hline $\begin{array}{l}\text { I only deliver information allowed at the student level by the CDR, as } \\
\text { specified in the Dietetic Scope of Practice. }\end{array}$ & 5.48 & 0.1400 \\
\hline I comprehend the scope of practice. & 3.71 & 0.4473 \\
\hline $\begin{array}{l}\text { I refer friends and family to other sources that are outside my scope of } \\
\text { practice. }\end{array}$ & 5.15 & 0.2722 \\
\hline I abide by the Dietetics Code of Ethics. & 5.13 & 0.1627 \\
\hline $\begin{array}{l}\text { I use nutrition information to diagnose health conditions in family } \\
\text { members and individuals. }\end{array}$ & 16.31 & 0.0026 \\
\hline Major & 0.18 & 0.6705 \\
\hline
\end{tabular}


Table 24 (Part A): OVERWEIGHT/OBESE: Get at least 60 minutes of exercise per day, most days of the week.

\begin{tabular}{|c|c|c|c|c|c|c|c|}
\hline \multicolumn{8}{|c|}{ OVERWEIGHT/OBESE: Get at least 60 minutes of exercise per day, most days of the week. } \\
\hline \multirow{5}{*}{$\begin{array}{l}\text { Parameter } \\
\text { I only deliver } \\
\text { information } \\
\text { allowed at the } \\
\text { student level by the } \\
\text { CDR, as specified in } \\
\text { the Dietetic Scope } \\
\text { of Practice. }\end{array}$} & \multirow{2}{*}{$\begin{array}{l}\text { Response } \\
\text { Rarely }\end{array}$} & \multirow{2}{*}{$\begin{array}{r}\text { Estimate } \\
24.9176\end{array}$} & \multirow{2}{*}{$\begin{array}{l}\text { Standard } \\
\text { Error }\end{array}$} & \multicolumn{2}{|c|}{$\begin{array}{l}\text { Wald 95\% } \\
\text { Confidence Limits }\end{array}$} & \multirow{2}{*}{\begin{tabular}{|r|}
$\begin{array}{l}\text { Wald } \\
\text { Chi-Square }\end{array}$ \\
164.40
\end{tabular}} & \multirow{2}{*}{\begin{tabular}{|l|}
$\begin{array}{l}\mathrm{Pr}> \\
\text { ChiSq }\end{array}$ \\
$<.0001$
\end{tabular}} \\
\hline & & & & 21.1087 & 28.7265 & & \\
\hline & Sometimes & -1.4151 & 1.1128 & -3.5961 & 0.7659 & 1.62 & 0.2035 \\
\hline & Often & 0.2591 & 1.1188 & -1.9338 & 2.4519 & 0.05 & 0.8169 \\
\hline & Always & 0.0000 & 0.0000 & 0.0000 & 0.0000 & & \\
\hline \multirow{5}{*}{$\begin{array}{l}\text { I comprehend the } \\
\text { scope of practice. }\end{array}$} & Never & -1.5534 & 2.1624 & -5.7916 & 2.6848 & 0.52 & 0.4725 \\
\hline & Rarely & -1.0277 & 1.5457 & -4.0573 & 2.0019 & 0.44 & 0.5061 \\
\hline & Sometimes & 1.7684 & 1.1064 & -0.4000 & 3.9368 & 2.55 & 0.1100 \\
\hline & Often & 0.0537 & 0.9339 & -1.7766 & 1.8841 & 0.00 & 0.9541 \\
\hline & Always & 0.0000 & 0.0000 & 0.0000 & 0.0000 & & \\
\hline \multirow{5}{*}{$\begin{array}{l}\text { I refer friends and } \\
\text { family to other } \\
\text { sources that are } \\
\text { outside my scope of } \\
\text { practice. }\end{array}$} & Never & -26.1385 & 2.2478 & -30.5442 & -21.7328 & 135.22 & $<.0001$ \\
\hline & Rarely & 25.0679 & 199240.4 & -390479 & 390529.1 & 0.00 & 0.9999 \\
\hline & Sometimes & 0.1519 & 0.8685 & -1.5503 & 1.8540 & 0.03 & 0.8612 \\
\hline & Often & 0.1824 & 0.7516 & -1.2907 & 1.6555 & 0.06 & 0.8083 \\
\hline & Always & 0.0000 & 0.0000 & 0.0000 & 0.0000 & & \\
\hline \multirow{4}{*}{$\begin{array}{l}\text { I abide by the } \\
\text { Dietetics Code of } \\
\text { Ethics. }\end{array}$} & Rarely & -29.0167 & 0.0000 & -29.0167 & -29.0167 & & \\
\hline & Sometimes & -0.1202 & 0.9385 & -1.9596 & 1.7192 & 0.02 & 0.8981 \\
\hline & Often & -0.6377 & 0.7598 & -2.1268 & 0.8514 & 0.70 & 0.4013 \\
\hline & Always & 0.0000 & 0.0000 & 0.0000 & 0.0000 & & \\
\hline \multirow{5}{*}{$\begin{array}{l}\text { I use nutrition } \\
\text { information to } \\
\text { diagnose health } \\
\text { conditions in family } \\
\text { members and } \\
\text { individuals. }\end{array}$} & Never & 22.5656 & 0.0000 & 22.5656 & 22.5656 & & \\
\hline & Rarely & -1.5384 & 1.3661 & -4.2158 & 1.1391 & 1.27 & 0.2601 \\
\hline & Sometimes & -3.7102 & 1.3993 & -6.4527 & -0.9676 & 7.03 & 0.0080 \\
\hline & Often & -2.2776 & 1.4770 & -5.1725 & 0.6173 & 2.38 & 0.1231 \\
\hline & Always & 0.0000 & 0.0000 & 0.0000 & 0.0000 & & \\
\hline \multirow{2}{*}{ Major } & Dietetics & -0.8658 & 0.6355 & -2.1113 & 0.3797 & 1.86 & 0.1730 \\
\hline & Human Ntr & 0.0000 & 0.0000 & 0.0000 & 0.0000 & & \\
\hline
\end{tabular}


Table 24 (Part B): OVERWEIGHT/OBESE: Get at least 60 minutes of exercise per day, most days of the week.

OVERWEIGHT/OBESE: Get at least 60 minutes of exercise per day, most days of the week.

\begin{tabular}{|l|r|r|}
\hline Source & Chi-Square & Pr > ChiSq \\
\hline $\begin{array}{l}\text { I only deliver information allowed at the student level by the CDR, as } \\
\text { specified in the Dietetic Scope of Practice. }\end{array}$ & 9.23 & 0.0264 \\
\hline I comprehend the scope of practice. & 11.12 & 0.0253 \\
\hline $\begin{array}{l}\text { I refer friends and family to other sources that are outside my scope of } \\
\text { practice. }\end{array}$ & 6.66 & 0.1553 \\
\hline I abide by the Dietetics Code of Ethics. & 8.85 & 0.0314 \\
\hline $\begin{array}{l}\text { I use nutrition information to diagnose health conditions in family } \\
\text { members and individuals. }\end{array}$ & 23.46 & 0.0001 \\
\hline Major & 1.93 & 0.1650 \\
\hline
\end{tabular}


Table 25: OVERWEIGHT/OBESE: Eat several smaller meals throughout the day instead of 3 large meals.

\begin{tabular}{|c|c|c|c|c|c|c|c|}
\hline \multicolumn{8}{|c|}{ OVERWEIGHT/OBESE: Eat several smaller meals throughout the day instead of 3 large meals. } \\
\hline \multirow{5}{*}{$\begin{array}{l}\text { Parameter } \\
\text { I only deliver } \\
\text { information } \\
\text { allowed at the } \\
\text { student level by the } \\
\text { CDR, as specified in } \\
\text { the Dietetic Scope } \\
\text { of Practice. }\end{array}$} & \multirow{2}{*}{\begin{tabular}{|l|} 
Response \\
Rarely \\
\end{tabular}} & \multirow{2}{*}{\begin{tabular}{|r|} 
Estimate \\
-6.1720
\end{tabular}} & \multirow{2}{*}{\begin{tabular}{|r|}
$\begin{array}{l}\text { Standard } \\
\text { Error }\end{array}$ \\
2.3341
\end{tabular}} & \multicolumn{2}{|c|}{$\begin{array}{l}\text { Wald 95\% } \\
\text { Confidence Limits }\end{array}$} & \multirow{2}{*}{\begin{tabular}{|r|}
$\mid \begin{array}{l}\text { Wald } \\
\text { Chi-Square }\end{array}$ \\
6.99 \\
\end{tabular}} & \multirow{2}{*}{ 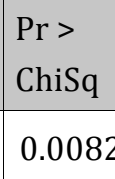 } \\
\hline & & & & -10.7468 & -1.5973 & & \\
\hline & Sometimes & -0.4717 & 0.9491 & -2.3318 & 1.3884 & 0.25 & 0.6192 \\
\hline & Often & 0.3082 & 0.9554 & -1.5642 & 2.1807 & 0.10 & 0.7470 \\
\hline & Always & 0.0000 & 0.0000 & 0.0000 & 0.0000 & & \\
\hline \multirow{5}{*}{$\begin{array}{l}\text { I comprehend the } \\
\text { scope of practice. }\end{array}$} & Never & 26.1972 & 266899.7 & -523088 & 523139.9 & 0.00 & 0.9999 \\
\hline & Rarely & 2.2264 & 1.6220 & -0.9526 & 5.4054 & 1.88 & 0.1699 \\
\hline & Sometimes & 1.3491 & 0.8938 & -0.4027 & 3.1009 & 2.28 & 0.1312 \\
\hline & Often & 0.8054 & 0.7666 & -0.6972 & 2.3079 & 1.10 & 0.2935 \\
\hline & Always & 0.0000 & 0.0000 & 0.0000 & 0.0000 & & \\
\hline \multirow{5}{*}{$\begin{array}{l}\text { I refer friends and } \\
\text { family to other } \\
\text { sources that are } \\
\text { outside my scope of } \\
\text { practice. }\end{array}$} & Never & 25.3176 & 266899.7 & -523088 & 523139.0 & 0.00 & 0.9999 \\
\hline & Rarely & 24.3495 & 182365.8 & -357406 & 357454.7 & 0.00 & 0.9999 \\
\hline & Sometimes & -0.2963 & 0.7060 & -1.6799 & 1.0874 & 0.18 & 0.6747 \\
\hline & Often & -0.5515 & 0.6248 & -1.7761 & 0.6730 & 0.78 & 0.3774 \\
\hline & Always & 0.0000 & 0.0000 & 0.0000 & 0.0000 & & \\
\hline \multirow{4}{*}{$\begin{array}{l}\text { I abide by the } \\
\text { Dietetics Code of } \\
\text { Ethics. }\end{array}$} & Rarely & 29.4374 & 188198.2 & -368832 & 368891.1 & 0.00 & 0.9999 \\
\hline & Sometimes & -1.3618 & 0.8434 & -3.0147 & 0.2912 & 2.61 & 0.1064 \\
\hline & Often & -1.6670 & 0.6869 & -3.0134 & -0.3206 & 5.89 & 0.0152 \\
\hline & Always & 0.0000 & 0.0000 & 0.0000 & 0.0000 & & \\
\hline \multirow{5}{*}{$\begin{array}{l}\text { I use nutrition } \\
\text { information to } \\
\text { diagnose health } \\
\text { conditions in family } \\
\text { members and } \\
\text { individuals. }\end{array}$} & Never & 0.6267 & 1.1146 & -1.5578 & 2.8112 & 0.32 & 0.5739 \\
\hline & Rarely & 1.0522 & 0.9252 & -0.7612 & 2.8657 & 1.29 & 0.2554 \\
\hline & Sometimes & 0.2169 & 0.8920 & -1.5313 & 1.9651 & 0.06 & 0.8079 \\
\hline & Often & 0.7441 & 0.9626 & -1.1426 & 2.6308 & 0.60 & 0.4395 \\
\hline & Always & 0.0000 & 0.0000 & 0.0000 & 0.0000 & & \\
\hline \multirow{2}{*}{ Major } & Dietetics & -0.5044 & 0.5370 & -1.5570 & 0.5481 & 0.88 & 0.3476 \\
\hline & Human Ntr & 0.0000 & 0.0000 & 0.0000 & 0.0000 & & \\
\hline
\end{tabular}


Table 26 (Part A): OVERWEIGHT/OBESE: Avoid eating or drinking anything after dinner.

\begin{tabular}{|c|c|c|c|c|c|c|c|}
\hline \multicolumn{8}{|c|}{ OVERWEIGHT/OBESE: Avoid eating or drinking anything after dinner. } \\
\hline \multirow{5}{*}{$\begin{array}{l}\text { Parameter } \\
\text { I only deliver } \\
\text { information allowed } \\
\text { at the student level } \\
\text { by the CDR, as } \\
\text { specified in the } \\
\text { Dietetic Scope of } \\
\text { Practice. }\end{array}$} & \multirow{2}{*}{$\begin{array}{l}\text { Response } \\
\text { Rarely }\end{array}$} & \multirow{2}{*}{$\begin{array}{r}\text { Estimate } \\
1.2346\end{array}$} & \multirow{2}{*}{\begin{tabular}{|r|}
$\begin{array}{l}\text { Standard } \\
\text { Error }\end{array}$ \\
2.9695
\end{tabular}} & \multicolumn{2}{|c|}{$\begin{array}{l}\text { Wald 95\% } \\
\text { Confidence Limits }\end{array}$} & \multirow{2}{*}{\begin{tabular}{|r} 
Wald \\
Chi-Square \\
0.17
\end{tabular}} & \multirow{2}{*}{$\begin{array}{l}\mathrm{Pr}> \\
\text { ChiSq }\end{array}$} \\
\hline & & & & -4.5855 & 7.0547 & & \\
\hline & Sometimes & 0.0383 & 0.7903 & -1.5107 & 1.5873 & 0.00 & 0.9614 \\
\hline & Often & -0.4604 & 0.8221 & -2.0717 & 1.1508 & 0.31 & 0.5754 \\
\hline & Always & 0.0000 & 0.0000 & 0.0000 & 0.0000 & & \\
\hline \multirow{5}{*}{$\begin{array}{l}\text { I comprehend the } \\
\text { scope of practice. }\end{array}$} & Never & -0.1865 & 1.7001 & -3.5187 & 3.1456 & 0.01 & 0.9126 \\
\hline & Rarely & 0.6423 & 1.3259 & -1.9564 & 3.2411 & 0.23 & 0.6281 \\
\hline & Sometimes & 1.3516 & 0.8199 & -0.2554 & 2.9587 & 2.72 & 0.0993 \\
\hline & Often & 1.6276 & 0.7444 & 0.1687 & 3.0866 & 4.78 & 0.0288 \\
\hline & Always & 0.0000 & 0.0000 & 0.0000 & 0.0000 & & \\
\hline \multirow{5}{*}{$\begin{array}{l}\text { I refer friends and } \\
\text { family to other } \\
\text { sources that are } \\
\text { outside my scope of } \\
\text { practice. }\end{array}$} & Never & 3.8387 & 1.9131 & 0.0891 & 7.5882 & 4.03 & 0.0448 \\
\hline & Rarely & 1.8957 & 1.5544 & -1.1509 & 4.9422 & 1.49 & 0.2226 \\
\hline & Sometimes & 0.7920 & 0.6789 & -0.5385 & 2.1226 & 1.36 & 0.2433 \\
\hline & Often & 0.6769 & 0.5670 & -0.4344 & 1.7882 & 1.43 & 0.2325 \\
\hline & Always & 0.0000 & 0.0000 & 0.0000 & 0.0000 & & \\
\hline \multirow{4}{*}{$\begin{array}{l}\text { I abide by the } \\
\text { Dietetics Code of } \\
\text { Ethics. }\end{array}$} & Rarely & -2.9295 & 3.3829 & -9.5598 & 3.7008 & 0.75 & 0.3865 \\
\hline & Sometimes & -0.6352 & 0.7616 & -2.1279 & 0.8574 & 0.70 & 0.4042 \\
\hline & Often & -0.8196 & 0.6196 & -2.0339 & 0.3948 & 1.75 & 0.1859 \\
\hline & Always & 0.0000 & 0.0000 & 0.0000 & 0.0000 & & \\
\hline \multirow{5}{*}{$\begin{array}{l}\text { I use nutrition } \\
\text { information to } \\
\text { diagnose health } \\
\text { conditions in family } \\
\text { members and } \\
\text { individuals. }\end{array}$} & Never & -2.4592 & 1.0138 & -4.4462 & -0.4721 & 5.88 & 0.0153 \\
\hline & Rarely & -1.5028 & 0.8207 & -3.1112 & 0.1057 & 3.35 & 0.0671 \\
\hline & Sometimes & -1.7337 & 0.7818 & -3.2659 & -0.2014 & 4.92 & 0.0266 \\
\hline & Often & -0.2078 & 0.8284 & -1.8315 & 1.4159 & 0.06 & 0.8019 \\
\hline & Always & 0.0000 & 0.0000 & 0.0000 & 0.0000 & & \\
\hline \multirow{2}{*}{ Major } & Dietetics & 0.0060 & 0.4856 & -0.9457 & 0.9578 & 0.00 & 0.9901 \\
\hline & Human Ntr & 0.0000 & 0.0000 & 0.0000 & 0.0000 & & \\
\hline
\end{tabular}


Table 26 (Part B): OVERWEIGHT/OBESE: Avoid eating or drinking anything after dinner.

\begin{tabular}{|l|r|r|}
\hline \multicolumn{3}{|c|}{ OVERWEIGHT/OBESE: Avoid eating or drinking anything after dinner. } \\
\hline Source & Chi-Square & Pr > ChiSq \\
\hline $\begin{array}{l}\text { I only deliver information allowed at the student level by the CDR, as } \\
\text { specified in the Dietetic Scope of Practice. }\end{array}$ & 1.04 & 0.7916 \\
\hline I comprehend the scope of practice. & 6.11 & 0.1910 \\
\hline $\begin{array}{l}\text { I refer friends and family to other sources that are outside my scope of } \\
\text { practice. }\end{array}$ & 5.39 & 0.2499 \\
\hline I abide by the Dietetics Code of Ethics. & 2.41 & 0.4925 \\
\hline $\begin{array}{l}\text { I use nutrition information to diagnose health conditions in family } \\
\text { members and individuals. }\end{array}$ & 15.86 & 0.0032 \\
\hline Major & 0.00 & 0.9901 \\
\hline
\end{tabular}


Table 27 (Part A): OVERWEIGHT/OBESE: Eat a high protein, high calorie diet.

\begin{tabular}{|c|c|c|c|c|c|c|c|}
\hline \multicolumn{8}{|c|}{ OVERWEIGHT/OBESE: Eat a high protein, high calorie diet. } \\
\hline \multirow{5}{*}{$\begin{array}{l}\text { Parameter } \\
\text { I only deliver } \\
\text { information } \\
\text { allowed at the } \\
\text { student level by the } \\
\text { CDR, as specified in } \\
\text { the Dietetic Scope } \\
\text { of Practice. }\end{array}$} & \multirow{2}{*}{$\begin{array}{l}\text { Response } \\
\text { Rarely }\end{array}$} & \multirow{2}{*}{\begin{tabular}{r|} 
Estimate \\
2.5810
\end{tabular}} & \multirow{2}{*}{$\begin{array}{l}\text { Standard } \\
\text { Error } \\
2.4128\end{array}$} & \multicolumn{2}{|c|}{$\begin{array}{l}\text { Wald 95\% } \\
\text { Confidence Limits }\end{array}$} & \multirow{2}{*}{$\begin{array}{l}\text { Wald } \\
\text { Chi-Square } \\
1.14\end{array}$} & \multirow{2}{*}{$\begin{array}{l}\operatorname{Pr}> \\
\text { ChiSq } \\
0.2847\end{array}$} \\
\hline & & & & -2.1479 & 7.3100 & & \\
\hline & Sometimes & -0.7580 & 1.0628 & -2.8410 & 1.3250 & 0.51 & 0.4757 \\
\hline & Often & -1.2839 & 1.1007 & -3.4413 & 0.8734 & 1.36 & 0.2434 \\
\hline & Always & 0.0000 & 0.0000 & 0.0000 & 0.0000 & & \\
\hline \multirow{5}{*}{$\begin{array}{l}\text { I comprehend the } \\
\text { scope of practice. }\end{array}$} & Never & -22.8100 & 107140.2 & -210014 & 209968.2 & 0.00 & 0.9998 \\
\hline & Rarely & 0.2691 & 1.8207 & -3.2994 & 3.8376 & 0.02 & 0.8825 \\
\hline & Sometimes & 2.4456 & 1.2348 & 0.0254 & 4.8659 & 3.92 & 0.0476 \\
\hline & Often & 2.7727 & 1.1402 & 0.5379 & 5.0075 & 5.91 & 0.0150 \\
\hline & Always & 0.0000 & 0.0000 & 0.0000 & 0.0000 & & \\
\hline \multirow{5}{*}{$\begin{array}{l}\text { I refer friends and } \\
\text { family to other } \\
\text { sources that are } \\
\text { outside my scope } \\
\text { of practice. }\end{array}$} & Never & 3.1466 & 2.1649 & -1.0965 & 7.3896 & 2.11 & 0.1461 \\
\hline & Rarely & 6.0679 & 1.9752 & 2.1966 & 9.9391 & 9.44 & 0.0021 \\
\hline & Sometimes & 1.8495 & 0.9550 & -0.0222 & 3.7212 & 3.75 & 0.0528 \\
\hline & Often & 1.8235 & 0.8285 & 0.1997 & 3.4473 & 4.84 & 0.0277 \\
\hline & Always & 0.0000 & 0.0000 & 0.0000 & 0.0000 & & \\
\hline \multirow{4}{*}{$\begin{array}{l}\text { I abide by the } \\
\text { Dietetics Code of } \\
\text { Ethics. }\end{array}$} & Rarely & -1.6548 & 2.3149 & -6.1920 & 2.8823 & 0.51 & 0.4747 \\
\hline & Sometimes & -2.3235 & 1.0033 & -4.2898 & -0.3571 & 5.36 & 0.0206 \\
\hline & Often & -2.1723 & 0.8617 & -3.8612 & -0.4833 & 6.35 & 0.0117 \\
\hline & Always & 0.0000 & 0.0000 & 0.0000 & 0.0000 & & \\
\hline \multirow{5}{*}{$\begin{array}{l}\text { I use nutrition } \\
\text { information to } \\
\text { diagnose health } \\
\text { conditions in } \\
\text { family members } \\
\text { and individuals. }\end{array}$} & Never & -1.6227 & 1.2317 & -4.0367 & 0.7913 & 1.74 & 0.1877 \\
\hline & Rarely & -3.1847 & 1.0860 & -5.3132 & -1.0563 & 8.60 & 0.0034 \\
\hline & Sometimes & -1.8250 & 0.9899 & -3.7652 & 0.1152 & 3.40 & 0.0652 \\
\hline & Often & -1.8093 & 1.0089 & -3.7867 & 0.1682 & 3.22 & 0.0729 \\
\hline & Always & 0.0000 & 0.0000 & 0.0000 & 0.0000 & & \\
\hline \multirow{2}{*}{ Major } & Dietetics & 0.7477 & 0.6178 & -0.4631 & 1.9585 & 1.46 & 0.2262 \\
\hline & Human Ntr & 0.0000 & 0.0000 & 0.0000 & 0.0000 & & \\
\hline
\end{tabular}


Table 27 (Part B): OVERWEIGHT/OBESE: Eat a high protein, high calorie diet.

\begin{tabular}{|l|r|r|}
\hline \multicolumn{3}{|c|}{ OVERWEIGHT/OBESE: Eat a high protein, high calorie diet. } \\
\hline Source & Chi-Square & $\operatorname{Pr}>$ ChiSq \\
\hline $\begin{array}{l}\text { I only deliver information allowed at the student level by the CDR, as } \\
\text { specified in the Dietetic Scope of Practice. }\end{array}$ & 3.89 & 0.2733 \\
\hline $\begin{array}{l}\text { I comprehend the scope of practice. } \\
\text { I refer friends and family to other sources that are outside my scope of } \\
\text { practice. }\end{array}$ & 11.37 & 0.0227 \\
\hline $\begin{array}{l}\text { I abide by the Dietetics Code of Ethics. } \\
\text { I use nutrition information to diagnose health conditions in family } \\
\text { members and individuals. }\end{array}$ & 7.13 & 0.0131 \\
\hline Major & 10.45 & 0.0677 \\
\hline
\end{tabular}


Table 28: OVERWEIGHT/OBESE: Avoid calorically dense foods and beverages.

\begin{tabular}{|c|c|c|c|c|c|c|c|}
\hline \multicolumn{8}{|c|}{ OVERWEIGHT/OBESE: Avoid calorically dense foods and beverages. } \\
\hline \multirow{5}{*}{$\begin{array}{l}\text { Parameter } \\
\text { I only deliver } \\
\text { information } \\
\text { allowed at the } \\
\text { student level by the } \\
\text { CDR, as specified in } \\
\text { the Dietetic Scope } \\
\text { of Practice. }\end{array}$} & \multirow{2}{*}{\begin{tabular}{|l} 
Response \\
Rarely \\
\end{tabular}} & \multirow{2}{*}{\begin{tabular}{r|} 
Estimate \\
-26.7622
\end{tabular}} & \multirow{2}{*}{$\begin{array}{l}\begin{array}{l}\text { Standard } \\
\text { Error }\end{array} \\
2.0023\end{array}$} & \multicolumn{2}{|c|}{$\begin{array}{l}\text { Wald 95\% } \\
\text { Confidence Limits }\end{array}$} & \multirow{2}{*}{\begin{tabular}{|r|}
$\begin{array}{l}\text { Wald } \\
\text { Chi-Square }\end{array}$ \\
178.64
\end{tabular}} & \multirow{2}{*}{ 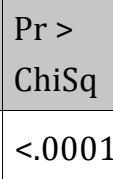 } \\
\hline & & & & -30.6866 & -22.8377 & & \\
\hline & Sometimes & 1.5657 & 0.9528 & -0.3017 & 3.4332 & 2.70 & 0.1003 \\
\hline & Often & 1.4546 & 0.9732 & -0.4527 & 3.3620 & 2.23 & 0.1350 \\
\hline & Always & 0.0000 & 0.0000 & 0.0000 & 0.0000 & & \\
\hline \multirow{5}{*}{$\begin{array}{l}\text { I comprehend the } \\
\text { scope of practice. }\end{array}$} & Never & 24.9513 & 267938.6 & -525125 & 525174.9 & 0.00 & 0.9999 \\
\hline & Rarely & 25.5944 & 2.5135 & 20.6680 & 30.5209 & 103.69 & $<.0001$ \\
\hline & Sometimes & -1.5320 & 0.8919 & -3.2800 & 0.2161 & 2.95 & 0.0859 \\
\hline & Often & -1.4635 & 0.8236 & -3.0778 & 0.1508 & 3.16 & 0.0756 \\
\hline & Always & 0.0000 & 0.0000 & 0.0000 & 0.0000 & & \\
\hline \multirow{5}{*}{$\begin{array}{l}\text { I refer friends and } \\
\text { family to other } \\
\text { sources that are } \\
\text { outside my scope } \\
\text { of practice. }\end{array}$} & Never & 24.4344 & 267938.6 & -525126 & 525174.4 & 0.00 & 0.9999 \\
\hline & Rarely & -2.4058 & 2.0163 & -6.3576 & 1.5460 & 1.42 & 0.2328 \\
\hline & Sometimes & -1.1993 & 0.7269 & -2.6240 & 0.2254 & 2.72 & 0.0990 \\
\hline & Often & -1.6025 & 0.6517 & -2.8798 & -0.3253 & 6.05 & 0.0139 \\
\hline & Always & 0.0000 & 0.0000 & 0.0000 & 0.0000 & & \\
\hline \multirow{4}{*}{$\begin{array}{l}\text { I abide by the } \\
\text { Dietetics Code of } \\
\text { Ethics. }\end{array}$} & Rarely & 25.9384 & 0.0000 & 25.9384 & 25.9384 & & \\
\hline & Sometimes & -0.2269 & 0.7960 & -1.7872 & 33 & 0.08 & 0.7756 \\
\hline & Often & 0.0964 & 0.6376 & -1.1534 & 1.3461 & 0.02 & 0.8799 \\
\hline & Always & 0.0000 & 0.0000 & 0.0000 & 0.0000 & & \\
\hline \multirow{5}{*}{$\begin{array}{l}\text { I use nutrition } \\
\text { information to } \\
\text { diagnose health } \\
\text { conditions in } \\
\text { family members } \\
\text { and individuals. }\end{array}$} & Never & 0.3951 & 1.1365 & -1.8324 & 2.6226 & 0.12 & 0.7281 \\
\hline & Rarely & 0.6311 & 0.8980 & -1.1289 & 2.3911 & 0.49 & 0.4822 \\
\hline & Sometimes & 0.0026 & 0.8845 & -1.7309 & 1.7362 & 0.00 & 0.9976 \\
\hline & Often & 1.2315 & 0.9649 & -0.6596 & 3.1227 & 1.63 & 0.2018 \\
\hline & Always & 0.0000 & 0.0000 & 0.0000 & 0.0000 & & \\
\hline \multirow{2}{*}{ Major } & Dietetics & 0.2963 & 0.5553 & -0.7920 & 1.3846 & 0.28 & 0.5936 \\
\hline & Human Ntr & 0.0000 & 0.0000 & 0.0000 & 0.0000 & & \\
\hline
\end{tabular}


Table 29 (Part A): OVERWEIGHT/OBESE: Consume less alcoholic beverages.

\begin{tabular}{|c|c|c|c|c|c|c|c|}
\hline \multicolumn{8}{|c|}{ OVERWEIGHT/OBESE: Consume less alcoholic beverages. } \\
\hline \multirow{5}{*}{$\begin{array}{l}\text { Parameter } \\
\text { I only deliver } \\
\text { information } \\
\text { allowed at the } \\
\text { student level by the } \\
\text { CDR, as specified in } \\
\text { the Dietetic Scope } \\
\text { of Practice. }\end{array}$} & \multirow{2}{*}{$\begin{array}{l}\text { Response } \\
\text { Rarely }\end{array}$} & \multirow{2}{*}{$\begin{array}{r}\text { Estimate } \\
0.3167\end{array}$} & \multirow{2}{*}{$\begin{array}{l}\text { Standard } \\
\text { Error } \\
2.3193\end{array}$} & \multicolumn{2}{|c|}{$\begin{array}{l}\text { Wald 95\% } \\
\text { Confidence Limits }\end{array}$} & \multirow{2}{*}{$\begin{array}{l}\text { Wald } \\
\text { Chi-Square } \\
0.02\end{array}$} & \multirow{2}{*}{\begin{tabular}{|l}
$\begin{array}{l}\text { Pr }> \\
\text { ChiSq }\end{array}$ \\
0.8914
\end{tabular}} \\
\hline & & & & -4.2290 & 4.8623 & & \\
\hline & Sometimes & 0.8983 & 0.8764 & -0.8194 & 2.6161 & 1.05 & 0.3054 \\
\hline & Often & 0.8913 & 0.8870 & -0.8472 & 2.6298 & 1.01 & 0.3150 \\
\hline & Always & 0.0000 & 0.0000 & 0.0000 & 0.0000 & & \\
\hline \multirow{5}{*}{$\begin{array}{l}\text { I comprehend the } \\
\text { scope of practice. }\end{array}$} & Never & 24.8656 & 90003.86 & -176379 & 176429.2 & 0.00 & 0.9998 \\
\hline & Rarely & -0.5284 & 1.3919 & -3.2566 & 2.1998 & 0.14 & 0.7042 \\
\hline & Sometimes & 0.7341 & 0.8440 & -0.9201 & 2.3883 & 0.76 & 0.3844 \\
\hline & Often & 1.0711 & 0.7677 & -0.4336 & 2.5758 & 1.95 & 0.1630 \\
\hline & Always & 0.0000 & 0.0000 & 0.0000 & 0.0000 & & \\
\hline \multirow{5}{*}{$\begin{array}{l}\text { I refer friends and } \\
\text { family to other } \\
\text { sources that are } \\
\text { outside my scope of } \\
\text { practice. }\end{array}$} & Never & -1.2983 & 1.9903 & -5.1993 & 2.6027 & 0.43 & 0.5142 \\
\hline & Rarely & -2.8675 & 1.7643 & -6.3253 & 0.5904 & 2.64 & 0.1041 \\
\hline & Sometimes & -0.4922 & 0.7249 & -1.9130 & 0.9287 & 0.46 & 0.4972 \\
\hline & Often & -1.5449 & 0.6179 & -2.7560 & -0.3338 & 6.25 & 0.0124 \\
\hline & Always & 0.0000 & 0.0000 & 0.0000 & 0.0000 & & \\
\hline \multirow{4}{*}{$\begin{array}{l}\text { I abide by the } \\
\text { Dietetics Code of } \\
\text { Ethics. }\end{array}$} & Rarely & 2.0594 & 2.5130 & -2.8659 & 6.9847 & 0.67 & 0.4125 \\
\hline & Sometimes & -1.5723 & 0.8112 & -3.1622 & 0.0175 & 3.76 & 0.0526 \\
\hline & Often & -0.8021 & 0.6515 & -2.0790 & 0.4747 & 1.52 & 0.2182 \\
\hline & Always & 0.0000 & 0.0000 & 0.0000 & 0.0000 & & \\
\hline \multirow{5}{*}{$\begin{array}{l}\text { I use nutrition } \\
\text { information to } \\
\text { diagnose health } \\
\text { conditions in family } \\
\text { members and } \\
\text { individuals. }\end{array}$} & Never & -1.0500 & 0.9997 & -3.0093 & 0.9094 & 1.10 & 0.2936 \\
\hline & Rarely & -1.0252 & 0.8307 & -2.6532 & 0.6029 & 1.52 & 0.2171 \\
\hline & Sometimes & -1.2380 & 0.8203 & -2.8458 & 0.3699 & 2.28 & 0.1313 \\
\hline & Often & -0.4356 & 0.8852 & -2.1706 & 1.2995 & 0.24 & 0.6227 \\
\hline & Always & 0.0000 & 0.0000 & 0.0000 & 0.0000 & & \\
\hline \multirow{2}{*}{ Major } & Dietetics & 1.0131 & 0.5710 & -0.1061 & 2.1324 & 3.15 & 0.0760 \\
\hline & Human Ntr & 0.0000 & 0.0000 & 0.0000 & 0.0000 & & \\
\hline
\end{tabular}


Table 29 (Part B): OVERWEIGHT/OBESE: Consume less alcoholic beverages.

\begin{tabular}{|l|r|r|}
\hline \multicolumn{3}{|c|}{ OVERWEIGHT/OBESE: Consume less alcoholic beverages. } \\
\hline Source & Chi-Square & Pr > ChiSq \\
\hline $\begin{array}{l}\text { I only deliver information allowed at the student level by the CDR, as } \\
\text { specified in the Dietetic Scope of Practice. }\end{array}$ & 1.19 & 0.7557 \\
\hline I comprehend the scope of practice. & 6.23 & 0.1825 \\
\hline $\begin{array}{l}\text { I refer friends and family to other sources that are outside my scope of } \\
\text { practice. }\end{array}$ & 8.38 & 0.0785 \\
\hline $\begin{array}{l}\text { I abide by the Dietetics Code of Ethics. } \\
\text { I use nutrition information to diagnose health conditions in family } \\
\text { members and individuals. }\end{array}$ & 3.89 & 0.1803 \\
\hline Major & 3.18 & 0.4321 \\
\hline
\end{tabular}


Table 30 (Part A): OVERWEIGHT/OBESE: Make smart food decisions when eating away from home.

\begin{tabular}{|c|c|c|c|c|c|c|c|}
\hline \multicolumn{8}{|c|}{ OVERWEIGHT/OBESE: Make smart food decisions when eating away from home. } \\
\hline \multirow{5}{*}{$\begin{array}{l}\text { Parameter } \\
\text { I only deliver } \\
\text { information } \\
\text { allowed at the } \\
\text { student level by the } \\
\text { CDR, as specified in } \\
\text { the Dietetic Scope } \\
\text { of Practice. }\end{array}$} & \multirow{2}{*}{\begin{tabular}{|l} 
Response \\
Rarely
\end{tabular}} & \multirow{2}{*}{\begin{tabular}{|r|} 
Estimate \\
20.8708 \\
\end{tabular}} & \multirow{2}{*}{\begin{tabular}{|r|}
$\begin{array}{l}\text { Standard } \\
\text { Error }\end{array}$ \\
1.9278 \\
\end{tabular}} & \multicolumn{2}{|c|}{$\begin{array}{l}\text { Wald 95\% } \\
\text { Confidence Limits }\end{array}$} & \multirow{2}{*}{\begin{tabular}{|r|}
$\begin{array}{l}\text { Wald } \\
\text { Chi-Square }\end{array}$ \\
117.21
\end{tabular}} & \multirow{2}{*}{$\begin{array}{l}\text { Pr }> \\
\text { ChiSq } \\
<.0001\end{array}$} \\
\hline & & & & 17.0924 & 24.6491 & & \\
\hline & Sometimes & 0.5084 & 1.1306 & -1.7074 & 2.7243 & 0.20 & 0.6529 \\
\hline & Often & -1.0201 & 1.1089 & -3.1934 & 1.1532 & 0.85 & 0.3576 \\
\hline & Always & 0.0000 & 0.0000 & 0.0000 & 0.0000 & & \\
\hline \multirow{5}{*}{$\begin{array}{l}\text { I comprehend the } \\
\text { scope of practice. }\end{array}$} & Never & 22.5406 & 196616.3 & -385338 & 385383.4 & 0.00 & 0.9999 \\
\hline & Rarely & -0.0386 & 1.9422 & -3.8453 & 3.7681 & 0.00 & 0.9842 \\
\hline & Sometimes & -0.5407 & 1.2327 & -2.9567 & 1.8753 & 0.19 & 0.6609 \\
\hline & Often & -0.7674 & 1.0546 & -2.8344 & 1.2996 & 0.53 & 0.4668 \\
\hline & Always & 0.0000 & 0.0000 & 0.0000 & 0.0000 & & \\
\hline \multirow{5}{*}{$\begin{array}{l}\text { I refer friends and } \\
\text { family to other } \\
\text { sources that are } \\
\text { outside my scope of } \\
\text { practice. }\end{array}$} & Never & 24.2510 & 196616.3 & -385337 & 385385.1 & 0.00 & 0.9999 \\
\hline & Rarely & -1.1287 & 1.9262 & -4.9040 & 2.6466 & 0.34 & 0.5579 \\
\hline & Sometimes & 0.5132 & 0.8841 & -1.2196 & 2.2461 & 0.34 & 0.5616 \\
\hline & Often & -0.0902 & 0.7453 & -1.5510 & 1.3706 & 0.01 & 0.9037 \\
\hline & Always & 0.0000 & 0.0000 & 0.0000 & 0.0000 & & \\
\hline \multirow{4}{*}{$\begin{array}{l}\text { I abide by the } \\
\text { Dietetics Code of } \\
\text { Ethics. }\end{array}$} & Rarely & -22.5360 & 0.0000 & -22.5360 & -22.5360 & & \\
\hline & Sometimes & -0.0126 & 1.0037 & -1.9799 & 1.9547 & 0.00 & 0.9900 \\
\hline & Often & -0.2284 & 0.8118 & -1.8196 & 1.3628 & 0.08 & 0.7784 \\
\hline & Always & 0.0000 & 0.0000 & 0.0000 & 0.0000 & & \\
\hline \multirow{5}{*}{$\begin{array}{l}\text { I use nutrition } \\
\text { information to } \\
\text { diagnose health } \\
\text { conditions in family } \\
\text { members and } \\
\text { individuals. }\end{array}$} & Never & -0.2472 & 1.2906 & -2.7767 & 2.2824 & 0.04 & 0.8481 \\
\hline & Rarely & 0.9128 & 1.1595 & -1.3598 & 3.1854 & 0.62 & 0.4311 \\
\hline & Sometimes & -0.9710 & 1.0127 & -2.9559 & 1.0140 & 0.92 & 0.3377 \\
\hline & Often & -0.5772 & 1.0750 & -2.6842 & 1.5298 & 0.29 & 0.5913 \\
\hline & Always & 0.0000 & 0.0000 & 0.0000 & 0.0000 & & \\
\hline \multirow{2}{*}{ Major } & Dietetics & 1.2967 & 0.6538 & 0.0154 & 2.5780 & 3.93 & 0.0473 \\
\hline & Human Ntr & 0.0000 & 0.0000 & 0.0000 & 0.0000 & & \\
\hline
\end{tabular}


Table 30 (Part B): OVERWEIGHT/OBESE: Make smart food decisions when eating away from home.

\begin{tabular}{|l|r|r|}
\hline \multicolumn{3}{|c|}{ OVERWEIGHT/OBESE: Make smart food decisions when eating away from home. } \\
\hline Source & Chi-Square & Pr > ChiSq \\
\hline $\begin{array}{l}\text { I only deliver information allowed at the student level by the CDR, as } \\
\text { specified in the Dietetic Scope of Practice. }\end{array}$ & 4.85 & 0.1830 \\
\hline I comprehend the scope of practice. & 1.18 & 0.8820 \\
\hline $\begin{array}{l}\text { I refer friends and family to other sources that are outside my scope of } \\
\text { practice. }\end{array}$ & 2.28 & 0.6840 \\
\hline $\begin{array}{l}\text { I abide by the Dietetics Code of Ethics. } \\
\text { I use nutrition information to diagnose health conditions in family } \\
\text { members and individuals. }\end{array}$ & 0.60 & 0.8965 \\
\hline Major & 4.19 & 0.1851 \\
\hline
\end{tabular}


Table 31 (Part A): OVERWEIGHT/OBESE: Use the Internet as a resource for nutrition facts at restaurants.

\begin{tabular}{|c|c|c|c|c|c|c|c|}
\hline \multicolumn{8}{|c|}{ OVERWEIGHT/OBESE: Use the Internet as a resource for nutrition facts at restaurants. } \\
\hline \multirow{5}{*}{\begin{tabular}{|l|} 
Parameter \\
I only deliver \\
information \\
allowed at the \\
student level by the \\
CDR, as specified in \\
the Dietetic Scope \\
of Practice.
\end{tabular}} & \multirow{2}{*}{\begin{tabular}{|l} 
Response \\
Rarely
\end{tabular}} & \multirow{2}{*}{\begin{tabular}{|r} 
Estimate \\
2.1365
\end{tabular}} & \multirow{2}{*}{\begin{tabular}{|r|}
$\begin{array}{l}\text { Standard } \\
\text { Error }\end{array}$ \\
2.1182 \\
\end{tabular}} & \multicolumn{2}{|c|}{$\begin{array}{l}\text { Wald 95\% } \\
\text { Confidence Limits }\end{array}$} & \multirow{2}{*}{\begin{tabular}{|r} 
Wald \\
Chi-Square \\
1.02
\end{tabular}} & \multirow{2}{*}{\begin{tabular}{|l|}
$\mathrm{Pr}>$ \\
ChiSq
\end{tabular}} \\
\hline & & & & -2.0151 & 6.2882 & & \\
\hline & Sometimes & 1.3971 & 0.9113 & -0.3890 & 3.1832 & 2.35 & 0.1252 \\
\hline & Often & 0.2491 & 0.9192 & -1.5525 & 2.0507 & 0.07 & 0.7864 \\
\hline & Always & 0.0000 & 0.0000 & 0.0000 & 0.0000 & & \\
\hline \multirow{5}{*}{$\begin{array}{l}\text { I comprehend the } \\
\text { scope of practice. }\end{array}$} & Never & 23.0174 & 151428.2 & -296771 & 296816.8 & 0.00 & 0.9999 \\
\hline & Rarely & -2.7164 & 1.5772 & -5.8077 & 0.3748 & 2.97 & 0.0850 \\
\hline & Sometimes & -1.4604 & 0.9086 & -3.2412 & 0.3205 & 2.58 & 0.1080 \\
\hline & Often & -0.6115 & 0.7926 & -2.1650 & 0.9420 & 0.60 & 0.4404 \\
\hline & Always & 0.0000 & 0.0000 & 0.0000 & 0.0000 & & \\
\hline \multirow{5}{*}{$\begin{array}{l}\text { I refer friends and } \\
\text { family to other } \\
\text { sources that are } \\
\text { outside my scope of } \\
\text { practice. }\end{array}$} & Never & 25.1479 & 151428.2 & -296769 & 296818.9 & 0.00 & 0.9999 \\
\hline & Rarely & -1.5080 & 1.6864 & -4.8133 & 1.7974 & 0.80 & 0.3712 \\
\hline & Sometimes & -0.5720 & 0.7080 & -1.9596 & 0.8155 & 0.65 & 0.4191 \\
\hline & Often & -0.6424 & 0.6173 & -1.8523 & 0.5676 & 1.08 & 0.2981 \\
\hline & Always & 0.0000 & 0.0000 & 0.0000 & 0.0000 & & \\
\hline \multirow{4}{*}{$\begin{array}{l}\text { I abide by the } \\
\text { Dietetics Code of } \\
\text { Ethics. }\end{array}$} & Rarely & -0.0707 & 2.1847 & -4.3526 & 4.2112 & 0.00 & 0.9742 \\
\hline & Sometimes & -0.7313 & 0.7874 & -2.2746 & 0.8120 & 0.86 & 0.3530 \\
\hline & Often & -0.6511 & 0.6563 & -1.9375 & 0.6353 & 0.98 & 0.3212 \\
\hline & Always & 0.0000 & 0.0000 & 0.0000 & 0.0000 & & \\
\hline \multirow{5}{*}{$\begin{array}{l}\text { I use nutrition } \\
\text { information to } \\
\text { diagnose health } \\
\text { conditions in family } \\
\text { members and } \\
\text { individuals. }\end{array}$} & Never & -1.3240 & 1.0755 & -3.4319 & 0.7839 & 1.52 & 0.2183 \\
\hline & Rarely & 0.5494 & 0.8862 & -1.1875 & 2.2862 & 0.38 & 0.5353 \\
\hline & Sometimes & -0.9618 & 0.8756 & -2.6779 & 0.7544 & 1.21 & 0.2720 \\
\hline & Often & -0.5426 & 0.9302 & -2.3657 & 1.2806 & 0.34 & 0.5597 \\
\hline & Always & 0.0000 & 0.0000 & 0.0000 & 0.0000 & & \\
\hline \multirow{2}{*}{ Major } & Dietetics & 1.0491 & 0.5119 & 0.0458 & 2.0524 & 4.20 & 0.0404 \\
\hline & Human Ntr & 0.0000 & 0.0000 & 0.0000 & 0.0000 & & \\
\hline
\end{tabular}


Table 31 (Part B): OVERWEIGHT/OBESE: Use the Internet as a resource for nutrition facts at restaurants.

OVERWEIGHT/OBESE: Use the Internet as a resource for nutrition facts at restaurants.

\begin{tabular}{|l|r|r|}
\hline Source & Chi-Square & Pr > ChiSq \\
\hline $\begin{array}{l}\text { I only deliver information allowed at the student level by the CDR, as } \\
\text { specified in the Dietetic Scope of Practice. }\end{array}$ & 5.27 & 0.1529 \\
\hline $\begin{array}{l}\text { I comprehend the scope of practice. } \\
\text { I refer friends and family to other sources that are outside my scope of } \\
\text { practice. }\end{array}$ & 4.96 & 0.2021 \\
\hline I abide by the Dietetics Code of Ethics. & 1.16 & 0.2945 \\
\hline $\begin{array}{l}\text { I use nutrition information to diagnose health conditions in family } \\
\text { members and individuals. }\end{array}$ & 8.04 & 0.0960 \\
\hline Major & 4.26 & 0.0389 \\
\hline
\end{tabular}


Table 32 (Part A): FRESHMAN 15: Eat a high protein, low carbohydrate diet.

\begin{tabular}{|c|c|c|c|c|c|c|c|}
\hline \multicolumn{8}{|c|}{ FRESHMAN 15: Eat a high protein, low carbohydrate diet. } \\
\hline \multirow{5}{*}{$\begin{array}{l}\text { Parameter } \\
\text { I only deliver } \\
\text { information } \\
\text { allowed at the } \\
\text { student level by the } \\
\text { CDR, as specified in } \\
\text { the Dietetic Scope } \\
\text { of Practice. }\end{array}$} & \multirow{2}{*}{$\begin{array}{l}\text { Response } \\
\text { Rarely }\end{array}$} & \multirow{2}{*}{$\begin{array}{r}\text { Estimate } \\
0.2193\end{array}$} & \multirow{2}{*}{\begin{tabular}{|l|l}
$\begin{array}{l}\text { Standard } \\
\text { Error }\end{array}$ \\
2.0736
\end{tabular}} & \multicolumn{2}{|c|}{$\begin{array}{l}\text { Wald 95\% } \\
\text { Confidence Limits }\end{array}$} & \multirow{2}{*}{\begin{tabular}{|r}
$\begin{array}{l}\text { Wald } \\
\text { Chi-Square }\end{array}$ \\
0.01
\end{tabular}} & \multirow{2}{*}{$\begin{array}{l}\begin{array}{l}\text { Pr }> \\
\text { ChiSq }\end{array} \\
0.9158\end{array}$} \\
\hline & & & & -3.8448 & 4.2834 & & \\
\hline & Sometimes & -1.1241 & 0.8687 & -2.8267 & 0.5785 & 1.67 & 0.1957 \\
\hline & Often & -0.3881 & 0.8881 & -2.1288 & 1.3527 & 0.19 & 0.6622 \\
\hline & Always & 0.0000 & 0.0000 & 0.0000 & 0.0000 & & \\
\hline \multirow{5}{*}{$\begin{array}{l}\text { I comprehend the } \\
\text { scope of practice. }\end{array}$} & Never & 1.5784 & 1.7736 & -1.8978 & 5.0547 & 0.79 & 0.3735 \\
\hline & Rarely & 0.6182 & 1.3538 & -2.0352 & 3.2716 & 0.21 & 0.6479 \\
\hline & Sometimes & 0.2406 & 0.8308 & -1.3878 & 1.8690 & 0.08 & 0.7721 \\
\hline & Often & 0.2754 & 0.7234 & -1.1424 & 1.6932 & 0.14 & 0.7034 \\
\hline & Always & 0.0000 & 0.0000 & 0.0000 & 0.0000 & & \\
\hline \multirow{5}{*}{$\begin{array}{l}\text { I refer friends and } \\
\text { family to other } \\
\text { sources that are } \\
\text { outside my scope of } \\
\text { practice. }\end{array}$} & Never & -22.0494 & 87054.23 & -170645 & 170601.1 & 0.00 & 0.9998 \\
\hline & Rarely & 0.5146 & 1.5283 & -2.4808 & 3.5100 & 0.11 & 0.7363 \\
\hline & Sometimes & 1.5739 & 0.7300 & 0.1432 & 3.0046 & 4.65 & 0.0311 \\
\hline & Often & 1.3010 & 0.6344 & 0.0577 & 2.5444 & 4.21 & 0.0403 \\
\hline & Always & 0.0000 & 0.0000 & 0.0000 & 0.0000 & & \\
\hline \multirow{4}{*}{$\begin{array}{l}\text { I abide by the } \\
\text { Dietetics Code of } \\
\text { Ethics. }\end{array}$} & Rarely & 1.5245 & 2.2405 & -2.8668 & 5.9157 & 0.46 & 0.4962 \\
\hline & Sometimes & -0.8357 & 0.8028 & -2.4092 & 0.7378 & 1.08 & 0.2979 \\
\hline & Often & 0.0483 & 0.6201 & -1.1671 & 1.2637 & 0.01 & 0.9379 \\
\hline & Always & 0.0000 & 0.0000 & 0.0000 & 0.0000 & & \\
\hline \multirow{5}{*}{$\begin{array}{l}\text { I use nutrition } \\
\text { information to } \\
\text { diagnose health } \\
\text { conditions in family } \\
\text { members and } \\
\text { individuals. }\end{array}$} & Never & -2.2335 & 1.0782 & -4.3466 & -0.1204 & 4.29 & 0.0383 \\
\hline & Rarely & -2.2882 & 0.8354 & -3.9255 & -0.6509 & 7.50 & 0.0062 \\
\hline & Sometimes & -1.1621 & 0.7915 & -2.7134 & 0.3892 & 2.16 & 0.1420 \\
\hline & Often & -0.6914 & 0.8709 & -2.3984 & 1.0156 & 0.63 & 0.4273 \\
\hline & Always & 0.0000 & 0.0000 & 0.0000 & 0.0000 & & \\
\hline \multirow{2}{*}{ Major } & Dietetics & -0.5331 & 0.5073 & -1.5274 & 0.4611 & 1.10 & 0.2933 \\
\hline & Human Ntr & 0.0000 & 0.0000 & 0.0000 & 0.0000 & & \\
\hline
\end{tabular}


Table 32 (Part B): FRESHMAN 15: Eat a high protein, low carbohydrate diet.

\begin{tabular}{|l|r|r|}
\hline \multicolumn{3}{|c|}{ FRESHMAN 15: Eat a high protein, low carbohydrate diet. } \\
\hline Source & Chi-Square & Pr > ChiSq \\
\hline $\begin{array}{l}\text { I only deliver information allowed at the student level by the CDR, as } \\
\text { specified in the Dietetic Scope of Practice. }\end{array}$ & 2.83 & 0.4184 \\
\hline I comprehend the scope of practice. & 0.90 & 0.9240 \\
\hline $\begin{array}{l}\text { I refer friends and family to other sources that are outside my scope of } \\
\text { practice. }\end{array}$ & 7.43 & 0.1147 \\
\hline I abide by the Dietetics Code of Ethics. & 2.76 & 0.4298 \\
\hline $\begin{array}{l}\text { I use nutrition information to diagnose health conditions in family } \\
\text { members and individuals. }\end{array}$ & 12.24 & 0.0156 \\
\hline Major & 1.11 & 0.2926 \\
\hline
\end{tabular}


Table 33: FRESHMAN 15: Get at least 60 minutes of exercise per day, most days of the week.

\begin{tabular}{|c|c|c|c|c|c|c|c|}
\hline \multicolumn{8}{|c|}{ FRESHMAN 15: Get at least 60 minutes of exercise per day, most days of the week. } \\
\hline \multirow{5}{*}{$\begin{array}{l}\text { Parameter } \\
\text { I only deliver } \\
\text { information } \\
\text { allowed at the } \\
\text { student level by the } \\
\text { CDR, as specified in } \\
\text { the Dietetic Scope } \\
\text { of Practice. }\end{array}$} & \multirow{2}{*}{$\begin{array}{l}\text { Response } \\
\text { Rarely }\end{array}$} & \multirow{2}{*}{\begin{tabular}{|r} 
Estimate \\
22.6029
\end{tabular}} & \multirow{2}{*}{\begin{tabular}{|r|}
$\begin{array}{l}\text { Standard } \\
\text { Error }\end{array}$ \\
1.9337
\end{tabular}} & \multicolumn{2}{|c|}{$\begin{array}{l}\text { Wald 95\% } \\
\text { Confidence Limits }\end{array}$} & \multirow{2}{*}{\begin{tabular}{|r}
$\begin{array}{l}\text { Wald } \\
\text { Chi-Square }\end{array}$ \\
136.63
\end{tabular}} & \multirow{2}{*}{$\begin{array}{l}\mathrm{Pr}> \\
\text { ChiSq }\end{array}$} \\
\hline & & & & 18.8129 & 26.3929 & & \\
\hline & Sometimes & -1.9871 & 1.0314 & -4.0087 & 0.0345 & 3.71 & 0.0540 \\
\hline & Often & -0.5280 & 1.0705 & -2.6261 & 1.5701 & 0.24 & 0.6218 \\
\hline & Always & 0.0000 & 0.0000 & 0.0000 & 0.0000 & & \\
\hline \multirow{5}{*}{$\begin{array}{l}\text { I comprehend the } \\
\text { scope of practice. }\end{array}$} & Never & -27.1061 & 219784.4 & -430797 & 430742.4 & 0.00 & 0.9999 \\
\hline & Rarely & -0.8215 & 1.4950 & -3.7516 & 2.1086 & 0.30 & 0.5827 \\
\hline & Sometimes & -2.3171 & 1.1083 & -4.4893 & -0.1450 & 4.37 & 0.0365 \\
\hline & Often & -2.0733 & 1.0138 & -4.0602 & -0.0864 & 4.18 & 0.0408 \\
\hline & Always & 0.0000 & 0.0000 & 0.0000 & 0.0000 & & \\
\hline \multirow{5}{*}{$\begin{array}{l}\text { I refer friends and } \\
\text { family to other } \\
\text { sources that are } \\
\text { outside my scope of } \\
\text { practice. }\end{array}$} & Never & 24.0420 & 286464.6 & -561436 & 561484.3 & 0.00 & 0.9999 \\
\hline & Rarely & -0.8610 & 1.7638 & -4.3180 & 2.5959 & 0.24 & 0.6254 \\
\hline & Sometimes & 1.0352 & 0.8441 & -0.6192 & 2.6896 & 1.50 & 0.2200 \\
\hline & Often & 0.0029 & 0.7100 & -1.3886 & 1.3945 & 0.00 & 0.9967 \\
\hline & Always & 0.0000 & 0.0000 & 0.0000 & 0.0000 & & \\
\hline \multirow{4}{*}{$\begin{array}{l}\text { I abide by the } \\
\text { Dietetics Code of } \\
\text { Ethics. }\end{array}$} & Rarely & -24.2027 & 0.0000 & -24.2027 & -24.2027 & & \\
\hline & Sometimes & 0.8503 & 0.9048 & -0.9231 & 2.6237 & 0.88 & 0.3473 \\
\hline & Often & 0.2410 & 0.7264 & -1.1828 & 1.6648 & 0.11 & 0.7401 \\
\hline & Always & 0.0000 & 0.0000 & 0.0000 & 0.0000 & & \\
\hline \multirow{5}{*}{$\begin{array}{l}\text { I use nutrition } \\
\text { information to } \\
\text { diagnose health } \\
\text { conditions in family } \\
\text { members and } \\
\text { individuals. }\end{array}$} & Never & 2.7070 & 1.3851 & -0.0078 & 5.4217 & 3.82 & 0.0507 \\
\hline & Rarely & 1.9336 & 0.9926 & -0.0119 & 3.8791 & 3.79 & 0.0514 \\
\hline & Sometimes & 1.0704 & 0.9419 & -0.7756 & 2.9164 & 1.29 & 0.2558 \\
\hline & Often & 1.4528 & 1.0037 & -0.5145 & 3.4201 & 2.09 & 0.1478 \\
\hline & Always & 0.0000 & 0.0000 & 0.0000 & 0.0000 & & \\
\hline \multirow{2}{*}{ Major } & Dietetics & -2.0206 & 0.6598 & -3.3137 & -0.7274 & 9.38 & 0.0022 \\
\hline & Human Ntr & 0.0000 & 0.0000 & 0.0000 & 0.0000 & & \\
\hline
\end{tabular}


Table 34 (Part A): FRESHMAN 15: Eat several smaller meals throughout the day instead of 3 large meals.

\begin{tabular}{|c|c|c|c|c|c|c|c|}
\hline \multicolumn{8}{|c|}{ FRESHMAN 15: Eat several smaller meals throughout the day instead of 3 large meals. } \\
\hline \multirow{5}{*}{$\begin{array}{l}\text { Parameter } \\
\text { I only deliver } \\
\text { information } \\
\text { allowed at the } \\
\text { student level by } \\
\text { the CDR, as } \\
\text { specified in the } \\
\text { Dietetic Scope of } \\
\text { Practice. }\end{array}$} & \multirow{2}{*}{$\begin{array}{l}\text { Response } \\
\text { Rarely }\end{array}$} & \multirow{2}{*}{$\begin{array}{r}\text { Estimate } \\
-6.5161\end{array}$} & \multirow{2}{*}{$\begin{array}{l}\text { Standard } \\
\text { Error } \\
2.1347\end{array}$} & \multicolumn{2}{|c|}{$\begin{array}{l}\text { Wald 95\% } \\
\text { Confidence Limits }\end{array}$} & \multirow{2}{*}{$\begin{array}{l}\text { Wald } \\
\text { Chi-Square } \\
\quad 9.32\end{array}$} & \multirow{2}{*}{$\begin{array}{l}\text { Pr > } \\
\text { ChiSq } \\
0.0023\end{array}$} \\
\hline & & & & -10.6999 & -2.3322 & & \\
\hline & Sometimes & -0.7533 & 0.9367 & -2.5892 & 1.0826 & 0.65 & 0.4213 \\
\hline & Often & -0.1599 & 0.9458 & -2.0136 & 1.6937 & 0.03 & 0.8657 \\
\hline & Always & 0.0000 & 0.0000 & 0.0000 & 0.0000 & & \\
\hline \multirow{5}{*}{$\begin{array}{l}\text { I comprehend the } \\
\text { scope of practice. }\end{array}$} & Never & 24.4795 & 91197.89 & -178720 & 178769.1 & 0.00 & 0.9998 \\
\hline & Rarely & 0.8101 & 1.3489 & -1.8337 & 3.4539 & 0.36 & 0.5481 \\
\hline & Sometimes & 1.2125 & 0.9016 & -0.5547 & 2.9797 & 1.81 & 0.1787 \\
\hline & Often & 1.0069 & 0.8075 & -0.5759 & 2.5896 & 1.55 & 0.2125 \\
\hline & Always & 0.0000 & 0.0000 & 0.0000 & 0.0000 & & \\
\hline \multirow{5}{*}{$\begin{array}{l}\text { I refer friends and } \\
\text { family to other } \\
\text { sources that are } \\
\text { outside my scope } \\
\text { of practice. }\end{array}$} & Never & -3.7001 & 1.9875 & -7.5956 & 0.1954 & 3.47 & 0.0627 \\
\hline & Rarely & -0.0344 & 1.6898 & -3.3464 & 3.2777 & 0.00 & 0.9838 \\
\hline & Sometimes & -0.3680 & 0.6987 & -1.7375 & 1.0014 & 0.28 & 0.5984 \\
\hline & Often & -1.4011 & 0.6300 & -2.6359 & -0.1664 & 4.95 & 0.0261 \\
\hline & Always & 0.0000 & 0.0000 & 0.0000 & 0.0000 & & \\
\hline \multirow{4}{*}{$\begin{array}{l}\text { I abide by the } \\
\text { Dietetics Code of } \\
\text { Ethics. }\end{array}$} & Rarely & 6.3688 & 2.2524 & 1.9542 & 10.7835 & 8.00 & 0.0047 \\
\hline & Sometimes & -1.1066 & 0.7920 & -2.6589 & 0.4456 & 1.95 & 0.1623 \\
\hline & Often & -1.6941 & 0.6604 & -2.9885 & -0.3998 & 6.58 & 0.0103 \\
\hline & Always & 0.0000 & 0.0000 & 0.0000 & 0.0000 & & \\
\hline \multirow{5}{*}{$\begin{array}{l}\text { I use nutrition } \\
\text { information to } \\
\text { diagnose health } \\
\text { conditions in } \\
\text { family members } \\
\text { and individuals. }\end{array}$} & Never & 1.0439 & 1.0848 & -1.0822 & 3.1699 & 0.93 & 0.3359 \\
\hline & Rarely & 0.6315 & 0.8488 & -1.0321 & 2.2951 & 0.55 & 0.4569 \\
\hline & Sometimes & 0.6910 & 0.8335 & -0.9426 & 2.3246 & 0.69 & 0.4071 \\
\hline & Often & 0.6951 & 0.8908 & -1.0508 & 2.4411 & 0.61 & 0.4352 \\
\hline & Always & 0.0000 & 0.0000 & 0.0000 & 0.0000 & & \\
\hline \multirow{2}{*}{ Major } & Dietetics & -0.0324 & 0.5278 & -1.0669 & 1.0020 & 0.00 & 0.9510 \\
\hline & Human Ntr & 0.0000 & 0.0000 & 0.0000 & 0.0000 & & \\
\hline
\end{tabular}


Table 34 (Part B): FRESHMAN 15: Eat several smaller meals throughout the day instead of 3 large meals.

FRESHMAN 15: Eat several smaller meals throughout the day instead of 3 large meals.

\begin{tabular}{|l|r|r|}
\hline Source & Chi-Square & Pr > ChiSq \\
\hline $\begin{array}{l}\text { I only deliver information allowed at the student level by the CDR, as } \\
\text { specified in the Dietetic Scope of Practice. }\end{array}$ & 10.94 & 0.0120 \\
\hline I comprehend the scope of practice. & 4.25 & 0.3727 \\
\hline $\begin{array}{l}\text { I refer friends and family to other sources that are outside my scope of } \\
\text { practice. }\end{array}$ & 8.80 & 0.0664 \\
\hline I abide by the Dietetics Code of Ethics. & 15.12 & 0.0017 \\
\hline $\begin{array}{l}\text { I use nutrition information to diagnose health conditions in family } \\
\text { members and individuals. }\end{array}$ & 1.03 & 0.9055 \\
\hline Major & 0.00 & 0.9510 \\
\hline
\end{tabular}


Table 35 (Part A): FRESHMAN 15: Avoid eating or drinking anything after dinner.

\begin{tabular}{|c|c|c|c|c|c|c|c|}
\hline \multicolumn{8}{|c|}{ FRESHMAN 15: Avoid eating or drinking anything after dinner. } \\
\hline \multirow{5}{*}{$\begin{array}{l}\text { Parameter } \\
\text { I only deliver } \\
\text { information allowed at } \\
\text { the student level by the } \\
\text { CDR, as specified in the } \\
\text { Dietetic Scope of } \\
\text { Practice. }\end{array}$} & \multirow{2}{*}{$\begin{array}{l}\text { Response } \\
\text { Rarely }\end{array}$} & \multirow{2}{*}{$\begin{array}{r}\text { Estimate } \\
1.8015\end{array}$} & \multirow{2}{*}{\begin{tabular}{|l|}
$\begin{array}{l}\text { Standard } \\
\text { Error }\end{array}$ \\
3.3191
\end{tabular}} & \multicolumn{2}{|c|}{$\begin{array}{l}\text { Wald 95\% } \\
\text { Confidence Limits }\end{array}$} & \multirow{2}{*}{\begin{tabular}{|r}
$\begin{array}{l}\text { Wald } \\
\text { Chi-Square }\end{array}$ \\
0.29
\end{tabular}} & \multirow{2}{*}{\begin{tabular}{|l} 
Pr $>$ \\
ChiSq
\end{tabular}} \\
\hline & & & & -4.7038 & 8.3068 & & \\
\hline & Sometimes & -0.0820 & 0.8202 & -1.6895 & 1.5256 & 0.01 & 0.9204 \\
\hline & Often & -0.6911 & 0.8231 & -2.3043 & 0.9221 & 0.70 & 0.4011 \\
\hline & Always & 0.0000 & 0.0000 & 0.0000 & 0.0000 & & \\
\hline \multirow{5}{*}{$\begin{array}{l}\text { I comprehend the } \\
\text { scope of practice. }\end{array}$} & Never & -1.8620 & 1.6954 & -5.1850 & 1.4609 & 1.21 & 0.2721 \\
\hline & Rarely & -1.2093 & 1.3698 & -3.8940 & 1.4754 & 0.78 & 0.3773 \\
\hline & Sometimes & -0.3248 & 0.8183 & -1.9287 & 1.2790 & 0.16 & 0.6914 \\
\hline & Often & 0.0455 & 0.7322 & -1.3896 & 1.4806 & 0.00 & 0.9504 \\
\hline & Always & 0.0000 & 0.0000 & 0.0000 & 0.0000 & & \\
\hline \multirow{5}{*}{$\begin{array}{l}\text { I refer friends and } \\
\text { family to other sources } \\
\text { that are outside my } \\
\text { scope of practice. }\end{array}$} & Never & 0.9467 & 1.7586 & -2.5001 & 4.3934 & 0.29 & 0.5904 \\
\hline & Rarely & 2.1651 & 2.1802 & -2.1080 & 6.4382 & 0.99 & 0.3207 \\
\hline & Sometimes & 0.6112 & 0.6772 & -0.7160 & 1.9384 & 0.81 & 0.3668 \\
\hline & Often & 0.4700 & 0.5650 & -0.6374 & 1.5773 & 0.69 & 0.4055 \\
\hline & Always & 0.0000 & 0.0000 & 0.0000 & 0.0000 & & \\
\hline \multirow{4}{*}{$\begin{array}{l}\text { I abide by the Dietetics } \\
\text { Code of Ethics. }\end{array}$} & Rarely & -2.2853 & 3.5966 & -9.3345 & 4.7639 & 0.40 & 0.5252 \\
\hline & Sometimes & -0.0038 & 0.7177 & -1.4105 & 1.4029 & 0.00 & 0.9957 \\
\hline & Often & -0.2987 & 0.5742 & -1.4242 & 0.8267 & 0.27 & 0.6029 \\
\hline & Always & 0.0000 & 0.0000 & 0.0000 & 0.0000 & & \\
\hline \multirow{5}{*}{$\begin{array}{l}\text { I use nutrition } \\
\text { information to } \\
\text { diagnose health } \\
\text { conditions in family } \\
\text { members and } \\
\text { individuals. }\end{array}$} & Never & -0.5991 & 0.9694 & -2.4991 & 1.3009 & 0.38 & 0.5365 \\
\hline & Rarely & -0.8899 & 0.7825 & -2.4236 & 0.6438 & 1.29 & 0.2554 \\
\hline & Sometimes & -0.3694 & 0.7510 & -1.8413 & 1.1026 & 0.24 & 0.6228 \\
\hline & Often & 0.5214 & 0.8084 & -1.0630 & 2.1059 & 0.42 & 0.5189 \\
\hline & Always & 0.0000 & 0.0000 & 0.0000 & 0.0000 & & \\
\hline \multirow{2}{*}{ Major } & Dietetics & 0.2452 & 0.4831 & -0.7017 & 1.1922 & 0.26 & 0.6118 \\
\hline & Human Ntr & 0.0000 & 0.0000 & 0.0000 & 0.0000 & & \\
\hline
\end{tabular}


Table 35 (Part B): FRESHMAN 15: Avoid eating or drinking anything after dinner.

\begin{tabular}{|l|r|r|}
\hline \multicolumn{3}{|c|}{ FRESHMAN 15: Avoid eating or drinking anything after dinner. } \\
\hline Source & Chi-Square & Pr > ChiSq \\
\hline $\begin{array}{l}\text { I only deliver information allowed at the student level by the CDR, as } \\
\text { specified in the Dietetic Scope of Practice. }\end{array}$ & 1.84 & 0.6054 \\
\hline $\begin{array}{l}\text { I comprehend the scope of practice. } \\
\begin{array}{l}\text { I refer friends and family to other sources that are outside my scope of } \\
\text { practice. }\end{array}\end{array}$ & 2.37 & 0.6682 \\
\hline $\begin{array}{l}\text { I abide by the Dietetics Code of Ethics. } \\
\text { I use nutrition information to diagnose health conditions in family } \\
\text { members and individuals. }\end{array}$ & 0.92 & 0.7659 \\
\hline Major & 6.52 & 0.8216 \\
\hline
\end{tabular}


Table 36 (Part A): FRESHMAN 15: Eat a high protein, high calorie diet.

\begin{tabular}{|c|c|c|c|c|c|c|c|}
\hline \multicolumn{8}{|c|}{ FRESHMAN 15: Eat a high protein, high calorie diet. } \\
\hline \multirow{5}{*}{$\begin{array}{l}\text { Parameter } \\
\text { I only deliver } \\
\text { information } \\
\text { allowed at the } \\
\text { student level by the } \\
\text { CDR, as specified in } \\
\text { the Dietetic Scope } \\
\text { of Practice. }\end{array}$} & \multirow{2}{*}{\begin{tabular}{|l} 
Response \\
Rarely
\end{tabular}} & \multirow{2}{*}{$\begin{array}{r}\text { Estimate } \\
3.1360\end{array}$} & \multirow{2}{*}{\begin{tabular}{|l}
$\begin{array}{l}\text { Standard } \\
\text { Error }\end{array}$ \\
2.3347
\end{tabular}} & \multicolumn{2}{|c|}{$\begin{array}{l}\text { Wald 95\% } \\
\text { Confidence Limits }\end{array}$} & \multirow{2}{*}{$\begin{array}{l}\text { Wald } \\
\text { Chi-Square } \\
1.80\end{array}$} & \multirow{2}{*}{$\begin{array}{l}\begin{array}{l}\text { Pr }> \\
\text { ChiSq }\end{array} \\
0.1792\end{array}$} \\
\hline & & & & -1.4400 & 7.7120 & & \\
\hline & Sometimes & -0.8189 & 1.0747 & -2.9252 & 1.2874 & 0.58 & 0.4460 \\
\hline & Often & -1.3068 & 1.1225 & -3.5069 & 0.8933 & 1.36 & 0.2444 \\
\hline & Always & 0.0000 & 0.0000 & 0.0000 & 0.0000 & & \\
\hline \multirow{5}{*}{$\begin{array}{l}\text { I comprehend the } \\
\text { scope of practice. }\end{array}$} & Never & -24.9899 & 172646.5 & -338406 & 338356.0 & 0.00 & 0.9999 \\
\hline & Rarely & -1.0203 & 1.6871 & -4.3270 & 2.2863 & 0.37 & 0.5453 \\
\hline & Sometimes & 0.6149 & 1.0950 & -1.5312 & 2.7610 & 0.32 & 0.5744 \\
\hline & Often & 1.3238 & 1.0134 & -0.6624 & 3.3100 & 1.71 & 0.1914 \\
\hline & Always & 0.0000 & 0.0000 & 0.0000 & 0.0000 & & \\
\hline \multirow{5}{*}{$\begin{array}{l}\text { I refer friends and } \\
\text { family to other } \\
\text { sources that are } \\
\text { outside my scope of } \\
\text { practice. }\end{array}$} & Never & -22.0914 & 172646.5 & -338403 & 338358.9 & 0.00 & 0.9999 \\
\hline & Rarely & 4.4960 & 1.8556 & 0.8591 & 8.1329 & 5.87 & 0.0154 \\
\hline & Sometimes & 1.8866 & 0.9349 & 0.0542 & 3.7189 & 4.07 & 0.0436 \\
\hline & Often & 1.7885 & 0.8291 & 0.1636 & 3.4135 & 4.65 & 0.0310 \\
\hline & Always & 0.0000 & 0.0000 & 0.0000 & 0.0000 & & \\
\hline \multirow{4}{*}{$\begin{array}{l}\text { I abide by the } \\
\text { Dietetics Code of } \\
\text { Ethics. }\end{array}$} & Rarely & -0.4931 & 2.2205 & -4.8451 & 3.8589 & 0.05 & 0.8243 \\
\hline & Sometimes & -2.6050 & 0.9401 & -4.4475 & -0.7625 & 7.68 & 0.0056 \\
\hline & Often & -1.4738 & 0.7605 & -2.9644 & 0.0167 & 3.76 & 0.0526 \\
\hline & Always & 0.0000 & 0.0000 & 0.0000 & 0.0000 & & \\
\hline \multirow{5}{*}{$\begin{array}{l}\text { I use nutrition } \\
\text { information to } \\
\text { diagnose health } \\
\text { conditions in family } \\
\text { members and } \\
\text { individuals. }\end{array}$} & Never & -1.0188 & 1.1893 & -3.3498 & 1.3121 & 0.73 & 0.3916 \\
\hline & Rarely & -2.4410 & 0.9981 & -4.3972 & -0.4848 & 5.98 & 0.0145 \\
\hline & Sometimes & -0.5468 & 0.8990 & -2.3089 & 1.2152 & 0.37 & 0.5430 \\
\hline & Often & -0.0956 & 0.9510 & -1.9595 & 1.7684 & 0.01 & 0.9200 \\
\hline & Always & 0.0000 & 0.0000 & 0.0000 & 0.0000 & & \\
\hline \multirow{2}{*}{ Major } & Dietetics & 0.1424 & 0.5873 & -1.0086 & 1.2935 & 0.06 & 0.8084 \\
\hline & Human Ntr & 0.0000 & 0.0000 & 0.0000 & 0.0000 & & \\
\hline
\end{tabular}


Table 36 (Part B): FRESHMAN 15: Eat a high protein, high calorie diet.

\begin{tabular}{|l|r|r|}
\hline \multicolumn{3}{|c|}{ FRESHMAN 15: Eat a high protein, high calorie diet. } \\
\hline Source & Chi-Square & Pr > ChiSq \\
\hline $\begin{array}{l}\text { I only deliver information allowed at the student level by the CDR, as } \\
\text { specified in the Dietetic Scope of Practice. }\end{array}$ & 4.68 & 0.1964 \\
\hline I comprehend the scope of practice. & 7.50 & 0.1119 \\
\hline $\begin{array}{l}\text { I refer friends and family to other sources that are outside my scope of } \\
\text { practice. }\end{array}$ & 9.71 & 0.0455 \\
\hline I abide by the Dietetics Code of Ethics. & 8.31 & 0.0400 \\
\hline $\begin{array}{l}\text { I use nutrition information to diagnose health conditions in family } \\
\text { members and individuals. }\end{array}$ & 11.85 & 0.0185 \\
\hline Major & 0.06 & 0.8083 \\
\hline
\end{tabular}


Table 37 (Part A): FRESHMAN 15: Avoid calorically dense foods and beverages.

\begin{tabular}{|c|c|c|c|c|c|c|c|}
\hline \multicolumn{8}{|c|}{ FRESHMAN 15: Avoid calorically dense foods and beverages. } \\
\hline \multirow{5}{*}{$\begin{array}{l}\text { Parameter } \\
\text { I only deliver } \\
\text { information } \\
\text { allowed at the } \\
\text { student level by the } \\
\text { CDR, as specified in } \\
\text { the Dietetic Scope } \\
\text { of Practice. }\end{array}$} & \multirow{2}{*}{$\begin{array}{l}\text { Response } \\
\text { Rarely }\end{array}$} & \multirow{2}{*}{$\begin{array}{r}\text { Estimate } \\
-1.6697\end{array}$} & \multirow{2}{*}{\begin{tabular}{|r|}
$\begin{array}{l}\text { Standard } \\
\text { Error }\end{array}$ \\
2.2190
\end{tabular}} & \multicolumn{2}{|c|}{$\begin{array}{l}\text { Wald 95\% } \\
\text { Confidence Limits }\end{array}$} & \multirow{2}{*}{\begin{tabular}{|r} 
Wald \\
Chi-Square
\end{tabular}} & \multirow{2}{*}{$\begin{array}{l}\begin{array}{l}\text { Pr }> \\
\text { ChiSq }\end{array} \\
0.4518\end{array}$} \\
\hline & & & & -6.0189 & 2.6795 & & \\
\hline & Sometimes & 0.6829 & 0.9402 & -1.1599 & 2.5257 & 0.53 & 0.4676 \\
\hline & Often & 1.5452 & 0.9716 & -0.3592 & 3.4495 & 2.53 & 0.1118 \\
\hline & Always & 0.0000 & 0.0000 & 0.0000 & 0.0000 & & \\
\hline \multirow{5}{*}{$\begin{array}{l}\text { I comprehend the } \\
\text { scope of practice. }\end{array}$} & Never & 24.5439 & 98558.04 & -193146 & 193194.8 & 0.00 & 0.9998 \\
\hline & Rarely & 2.2913 & 1.6198 & -0.8835 & 5.4661 & 2.00 & 0.1572 \\
\hline & Sometimes & -0.3494 & 0.8481 & -2.0116 & 1.3129 & 0.17 & 0.6804 \\
\hline & Often & -0.5349 & 0.7796 & -2.0628 & 0.9930 & 0.47 & 0.4926 \\
\hline & Always & 0.0000 & 0.0000 & 0.0000 & 0.0000 & & \\
\hline \multirow{5}{*}{$\begin{array}{l}\text { I refer friends and } \\
\text { family to other } \\
\text { sources that are } \\
\text { outside my scope of } \\
\text { practice. }\end{array}$} & Never & -2.2245 & 2.0666 & -6.2750 & 1.8261 & 1.16 & 0.2818 \\
\hline & Rarely & -2.7522 & 1.8630 & -6.4037 & 0.8992 & 2.18 & 0.1396 \\
\hline & Sometimes & -1.3541 & 0.7237 & -2.7725 & 0.0643 & 3.50 & 0.0613 \\
\hline & Often & -1.6045 & 0.6518 & -2.8820 & -0.3271 & 6.06 & 0.0138 \\
\hline & Always & 0.0000 & 0.0000 & 0.0000 & 0.0000 & & \\
\hline \multirow{4}{*}{$\begin{array}{l}\text { I abide by the } \\
\text { Dietetics Code of } \\
\text { Ethics. }\end{array}$} & Rarely & 1.3037 & 2.2262 & -3.0596 & 5.6670 & 0.34 & 0.5581 \\
\hline & Sometimes & 0.3456 & 0.7866 & -1.1961 & 1.8874 & 0.19 & 0.6604 \\
\hline & Often & -0.2961 & 0.6326 & -1.5359 & 0.9437 & 0.22 & 0.6397 \\
\hline & Always & 0.0000 & 0.0000 & 0.0000 & 0.0000 & & \\
\hline \multirow{5}{*}{$\begin{array}{l}\text { I use nutrition } \\
\text { information to } \\
\text { diagnose health } \\
\text { conditions in family } \\
\text { members and } \\
\text { individuals. }\end{array}$} & Never & 0.6925 & 1.0970 & -1.4576 & 2.8426 & 0.40 & 0.5279 \\
\hline & Rarely & 0.6671 & 0.9114 & -1.1192 & 2.4534 & 0.54 & 0.4642 \\
\hline & Sometimes & 0.0037 & 0.9074 & -1.7746 & 1.7821 & 0.00 & 0.9967 \\
\hline & Often & 1.0268 & 0.9854 & -0.9046 & 2.9582 & 1.09 & 0.2974 \\
\hline & Always & 0.0000 & 0.0000 & 0.0000 & 0.0000 & & \\
\hline \multirow{2}{*}{ Major } & Dietetics & 0.2543 & 0.5438 & -0.8114 & 1.3201 & 0.22 & 0.6400 \\
\hline & Human Ntr & 0.0000 & 0.0000 & 0.0000 & 0.0000 & & \\
\hline
\end{tabular}


Table 37 (Part B): FRESHMAN 15: Avoid calorically dense foods and beverages.

\begin{tabular}{|l|r|r|}
\hline \multicolumn{3}{|c|}{ FRESHMAN 15: Avoid calorically dense foods and beverages. } \\
\hline Source & Chi-Square & Pr > ChiSq \\
\hline $\begin{array}{l}\text { I only deliver information allowed at the student level by the CDR, as } \\
\text { specified in the Dietetic Scope of Practice. }\end{array}$ & 4.79 & 0.1878 \\
\hline $\begin{array}{l}\text { I comprehend the scope of practice. } \\
\text { I refer friends and family to other sources that are outside my scope of } \\
\text { practice. }\end{array}$ & 7.39 & 0.0784 \\
\hline $\begin{array}{l}\text { I abide by the Dietetics Code of Ethics. } \\
\text { I use nutrition information to diagnose health conditions in family } \\
\text { members and individuals. }\end{array}$ & 1.26 & 0.1165 \\
\hline Major & 3.45 & 0.48679 \\
\hline
\end{tabular}


Table 38: FRESHMAN 15: Consume less alcoholic beverages.

\begin{tabular}{|c|c|c|c|c|c|c|c|}
\hline \multicolumn{8}{|c|}{ FRESHMAN 15: Consume less alcoholic beverages. } \\
\hline \multirow{5}{*}{$\begin{array}{l}\text { Parameter } \\
\text { I only deliver } \\
\text { information allowed } \\
\text { at the student level } \\
\text { by the CDR, as } \\
\text { specified in the } \\
\text { Dietetic Scope of } \\
\text { Practice. }\end{array}$} & \multirow{2}{*}{$\begin{array}{l}\text { Response } \\
\text { Rarely }\end{array}$} & \multirow{2}{*}{$\begin{array}{r}\text { Estimate } \\
0.9193\end{array}$} & \multirow{2}{*}{$\begin{array}{l}\text { Standard } \\
\text { Error } \\
2.3406\end{array}$} & \multicolumn{2}{|c|}{$\begin{array}{l}\text { Wald 95\% } \\
\text { Confidence Limits }\end{array}$} & \multirow{2}{*}{$\begin{array}{l}\text { Wald } \\
\text { Chi-Square } \\
0.15\end{array}$} & \multirow{2}{*}{\begin{tabular}{|l}
$\begin{array}{l}\text { Pr }> \\
\text { ChiSq }\end{array}$ \\
0.6945
\end{tabular}} \\
\hline & & & & -3.6682 & 5.5068 & & \\
\hline & Sometimes & 1.9388 & 0.9083 & 0.1585 & 3.7190 & 4.56 & 0.0328 \\
\hline & Often & 1.8895 & 0.9362 & 0.0546 & 3.7244 & 4.07 & 0.0436 \\
\hline & Always & 0.0000 & 0.0000 & 0.0000 & 0.0000 & & \\
\hline \multirow{5}{*}{$\begin{array}{l}\text { I comprehend the } \\
\text { scope of practice. }\end{array}$} & Never & 26.1138 & 254563.6 & -498909 & 498961.5 & 0.00 & 0.9999 \\
\hline & Rarely & -0.6062 & 1.5734 & -3.6900 & 2.4776 & 0.15 & 0.7000 \\
\hline & Sometimes & -0.7715 & 0.8817 & -2.4997 & 0.9566 & 0.77 & 0.3816 \\
\hline & Often & -0.3574 & 0.8172 & -1.9592 & 1.2443 & 0.19 & 0.6618 \\
\hline & Always & 0.0000 & 0.0000 & 0.0000 & 0.0000 & & \\
\hline \multirow{5}{*}{$\begin{array}{l}\text { I refer friends and } \\
\text { family to other } \\
\text { sources that are } \\
\text { outside my scope of } \\
\text { practice. }\end{array}$} & Never & -2.2679 & 2.0688 & -6.3226 & 1.7868 & 1.20 & 0.2730 \\
\hline & Rarely & 24.4199 & 179469.6 & -351730 & 351778.4 & 0.00 & 0.9999 \\
\hline & Sometimes & -2.0815 & 0.7624 & -3.5757 & -0.5873 & 7.45 & 0.0063 \\
\hline & Often & -1.7780 & 0.6320 & -3.0166 & -0.5394 & 7.92 & 0.0049 \\
\hline & Always & 0.0000 & 0.0000 & 0.0000 & 0.0000 & & \\
\hline \multirow{4}{*}{$\begin{array}{l}\text { I abide by the } \\
\text { Dietetics Code of } \\
\text { Ethics. }\end{array}$} & Rarely & 28.4272 & 175154.6 & -343268 & 343325.0 & 0.00 & 0.9999 \\
\hline & Sometimes & -0.6392 & 0.7990 & -2.2052 & 0.9268 & 0.64 & 0.4237 \\
\hline & Often & 0.5333 & 0.6453 & -0.7314 & 1.7980 & 0.68 & 0.4085 \\
\hline & Always & 0.0000 & 0.0000 & 0.0000 & 0.0000 & & \\
\hline \multirow{5}{*}{$\begin{array}{l}\text { I use nutrition } \\
\text { information to } \\
\text { diagnose health } \\
\text { conditions in family } \\
\text { members and } \\
\text { individuals. }\end{array}$} & Never & -0.3362 & 1.0376 & -2.3698 & 1.6974 & 0.11 & 0.7459 \\
\hline & Rarely & -0.6929 & 0.8918 & -2.4408 & 1.0550 & 0.60 & 0.4372 \\
\hline & Sometimes & -0.4759 & 0.8655 & -2.1723 & 1.2204 & 0.30 & 0.5824 \\
\hline & Often & 0.6120 & 0.9329 & -1.2165 & 2.4405 & 0.43 & 0.5118 \\
\hline & Always & 0.0000 & 0.0000 & 0.0000 & 0.0000 & & \\
\hline \multirow{2}{*}{ Major } & Dietetics & 0.8297 & 0.5786 & -0.3043 & 1.9637 & 2.06 & 0.1516 \\
\hline & Human Ntr & 0.0000 & 0.0000 & 0.0000 & 0.0000 & & \\
\hline
\end{tabular}


Table 39: FRESHMAN 15: Make smart food decisions when eating away from home.

\begin{tabular}{|c|c|c|c|c|c|c|c|}
\hline \multicolumn{8}{|c|}{ FRESHMAN 15: Make smart food decisions when eating away from home. } \\
\hline \multirow{5}{*}{$\begin{array}{l}\text { Parameter } \\
\text { I only deliver } \\
\text { information } \\
\text { allowed at the } \\
\text { student level by the } \\
\text { CDR, as specified in } \\
\text { the Dietetic Scope } \\
\text { of Practice. }\end{array}$} & \multirow{2}{*}{\begin{tabular}{|l} 
Response \\
Rarely
\end{tabular}} & \multirow{2}{*}{\begin{tabular}{|r|} 
Estimate \\
-1.5118
\end{tabular}} & \multirow{2}{*}{ 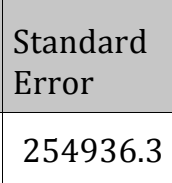 } & \multicolumn{2}{|c|}{$\begin{array}{l}\text { Wald 95\% } \\
\text { Confidence Limits }\end{array}$} & \multirow{2}{*}{\begin{tabular}{|l} 
Wald \\
Chi-Square \\
0.00
\end{tabular}} & \multirow{2}{*}{$\begin{array}{l}\text { Pr }> \\
\text { ChiSq }\end{array}$} \\
\hline & & & & -499667 & 499664.4 & & \\
\hline & Sometimes & 0.4475 & 1.0142 & -1.5404 & 2.4353 & 0.19 & 0.6591 \\
\hline & Often & -0.4734 & 1.0666 & -2.5640 & 1.6171 & 0.20 & 0.6572 \\
\hline & Always & 0.0000 & 0.0000 & 0.0000 & 0.0000 & & \\
\hline \multirow{5}{*}{$\begin{array}{l}\text { I comprehend the } \\
\text { scope of practice. }\end{array}$} & Never & 22.6611 & 308111.9 & -603866 & 603910.8 & 0.00 & 0.9999 \\
\hline & Rarely & 22.6456 & 133293.6 & -261228 & 261273.3 & 0.00 & 0.9999 \\
\hline & Sometimes & -3.1838 & 1.3311 & -5.7927 & -0.5750 & 5.72 & 0.0168 \\
\hline & Often & -2.3579 & 1.2158 & -4.7408 & 0.0250 & 3.76 & 0.0525 \\
\hline & Always & 0.0000 & 0.0000 & 0.0000 & 0.0000 & & \\
\hline \multirow{5}{*}{$\begin{array}{l}\text { I refer friends and } \\
\text { family to other } \\
\text { sources that are } \\
\text { outside my scope of } \\
\text { practice. }\end{array}$} & Never & 24.2296 & 308111.9 & -603864 & 603912.4 & 0.00 & 0.9999 \\
\hline & Rarely & -2.3371 & 2.0379 & -6.3314 & 1.6572 & 1.32 & 0.2515 \\
\hline & Sometimes & -0.5412 & 0.8311 & -2.1702 & 1.0878 & 0.42 & 0.5149 \\
\hline & Often & -0.7189 & 0.7048 & -2.1003 & 0.6625 & 1.04 & 0.3077 \\
\hline & Always & 0.0000 & 0.0000 & 0.0000 & 0.0000 & & \\
\hline \multirow{4}{*}{$\begin{array}{l}\text { I abide by the } \\
\text { Dietetics Code of } \\
\text { Ethics. }\end{array}$} & Rarely & 26.0884 & 319111.2 & -625420 & 625472.5 & 0.00 & 0.9999 \\
\hline & Sometimes & 0.8839 & 0.9385 & -0.9556 & 2.7234 & 0.89 & 0.3463 \\
\hline & Often & 0.5618 & 0.7531 & -0.9143 & 2.0380 & 0.56 & 0.4557 \\
\hline & Always & 0.0000 & 0.0000 & 0.0000 & 0.0000 & & \\
\hline \multirow{5}{*}{$\begin{array}{l}\text { I use nutrition } \\
\text { information to } \\
\text { diagnose health } \\
\text { conditions in family } \\
\text { members and } \\
\text { individuals. }\end{array}$} & Never & 3.0210 & 1.2590 & 0.5533 & 5.4887 & 5.76 & 0.0164 \\
\hline & Rarely & 3.6856 & 1.1174 & 1.4957 & 5.8756 & 10.88 & 0.0010 \\
\hline & Sometimes & 2.5916 & 1.0101 & 0.6118 & 4.5713 & 6.58 & 0.0103 \\
\hline & Often & 2.7164 & 1.0408 & 0.6766 & 4.7563 & 6.81 & 0.0091 \\
\hline & Always & 0.0000 & 0.0000 & 0.0000 & 0.0000 & & \\
\hline \multirow{2}{*}{ Major } & Dietetics & 0.3248 & 0.6042 & -0.8593 & 1.5090 & 0.29 & 0.5908 \\
\hline & Human Ntr & 0.0000 & 0.0000 & 0.0000 & 0.0000 & & \\
\hline
\end{tabular}


Table 40 (Part A): FRESHMAN 15: Use the Internet as a resource for nutrition facts at restaurants.

\begin{tabular}{|c|c|c|c|c|c|c|c|}
\hline \multicolumn{8}{|c|}{ FRESHMAN 15: Use the Internet as a resource for nutrition facts at restaurants. } \\
\hline \multirow{5}{*}{$\begin{array}{l}\text { Parameter } \\
\text { I only deliver } \\
\text { information allowed } \\
\text { at the student level } \\
\text { by the CDR, as } \\
\text { specified in the } \\
\text { Dietetic Scope of } \\
\text { Practice. }\end{array}$} & \multirow{2}{*}{$\begin{array}{l}\text { Response } \\
\text { Rarely }\end{array}$} & \multirow{2}{*}{$\begin{array}{r}\text { Estimate } \\
1.8407\end{array}$} & \multirow{2}{*}{\begin{tabular}{|l|l}
$\begin{array}{l}\text { Standard } \\
\text { Error }\end{array}$ \\
2.0876
\end{tabular}} & \multicolumn{2}{|c|}{$\begin{array}{l}\text { Wald 95\% } \\
\text { Confidence Limits }\end{array}$} & \multirow{2}{*}{\begin{tabular}{|r}
$\begin{array}{l}\text { Wald } \\
\text { Chi-Square }\end{array}$ \\
0.78
\end{tabular}} & \multirow{2}{*}{$\begin{array}{l}\begin{array}{l}\text { Pr }> \\
\text { ChiSq }\end{array} \\
0.3779\end{array}$} \\
\hline & & & & -2.2508 & 5.9323 & & \\
\hline & Sometimes & 1.4351 & 0.8893 & -0.3079 & 3.1780 & 2.60 & 0.1066 \\
\hline & Often & 0.6412 & 0.9172 & -1.1565 & 2.4389 & 0.49 & 0.4845 \\
\hline & Always & 0.0000 & 0.0000 & 0.0000 & 0.0000 & & \\
\hline \multirow{5}{*}{$\begin{array}{l}\text { I comprehend the } \\
\text { scope of practice. }\end{array}$} & Never & 21.9290 & 91613.75 & -179538 & 179581.6 & 0.00 & 0.9998 \\
\hline & Rarely & -2.1011 & 1.5593 & -5.1572 & 0.9550 & 1.82 & 0.1778 \\
\hline & Sometimes & -2.6956 & 0.9310 & -4.5203 & -0.8710 & 8.38 & 0.0038 \\
\hline & Often & -1.0250 & 0.7771 & -2.5481 & 0.4981 & 1.74 & 0.1872 \\
\hline & Always & 0.0000 & 0.0000 & 0.0000 & 0.0000 & & \\
\hline \multirow{5}{*}{$\begin{array}{l}\text { I refer friends and } \\
\text { family to other } \\
\text { sources that are } \\
\text { outside my scope of } \\
\text { practice. }\end{array}$} & Never & -2.8490 & 1.9443 & -6.6598 & 0.9617 & 2.15 & 0.1428 \\
\hline & Rarely & -1.6535 & 1.6154 & -4.8197 & 1.5127 & 1.05 & 0.3060 \\
\hline & Sometimes & -0.5641 & 0.6925 & -1.9214 & 0.7932 & 0.66 & 0.4153 \\
\hline & Often & -0.9037 & 0.6091 & -2.0976 & 0.2902 & 2.20 & 0.1379 \\
\hline & Always & 0.0000 & 0.0000 & 0.0000 & 0.0000 & & \\
\hline \multirow{4}{*}{$\begin{array}{l}\text { I abide by the } \\
\text { Dietetics Code of } \\
\text { Ethics. }\end{array}$} & Rarely & 1.5075 & 2.1520 & -2.7103 & 5.7252 & 0.49 & 0.4836 \\
\hline & Sometimes & -0.1563 & 0.8012 & -1.7267 & 1.4142 & 0.04 & 0.8454 \\
\hline & Often & -0.0370 & 0.6743 & -1.3585 & 1.2845 & 0.00 & 0.9563 \\
\hline & Always & 0.0000 & 0.0000 & 0.0000 & 0.0000 & & \\
\hline \multirow{5}{*}{$\begin{array}{l}\text { I use nutrition } \\
\text { information to } \\
\text { diagnose health } \\
\text { conditions in family } \\
\text { members and } \\
\text { individuals. }\end{array}$} & Never & 0.6976 & 0.9916 & -1.2459 & 2.6411 & 0.49 & 0.4817 \\
\hline & Rarely & 1.4771 & 0.8317 & -0.1530 & 3.1072 & 3.15 & 0.0757 \\
\hline & Sometimes & 0.9652 & 0.7983 & -0.5995 & 2.5299 & 1.46 & 0.2267 \\
\hline & Often & 1.2831 & 0.8698 & -0.4217 & 2.9879 & 2.18 & 0.1402 \\
\hline & Always & 0.0000 & 0.0000 & 0.0000 & 0.0000 & & \\
\hline \multirow{2}{*}{ Major } & Dietetics & 0.3645 & 0.5051 & -0.6255 & 1.3546 & 0.52 & 0.4705 \\
\hline & Human Ntr & 0.0000 & 0.0000 & 0.0000 & 0.0000 & & \\
\hline
\end{tabular}


Table 40 (Part B): FRESHMAN 15: Use the Internet as a resource for nutrition facts at restaurants.

\begin{tabular}{|l|r|r|}
\hline \multicolumn{3}{|c|}{ FRESHMAN 15: Use the Internet as a resource for nutrition facts at restaurants. } \\
\hline Source & Chi-Square & $\mathrm{Pr}>$ ChiSq \\
\hline $\begin{array}{l}\text { I only deliver information allowed at the student level by the CDR, as } \\
\text { specified in the Dietetic Scope of Practice. }\end{array}$ & 3.73 & 0.2919 \\
\hline I comprehend the scope of practice. & 12.95 & 0.0115 \\
\hline $\begin{array}{l}\text { I refer friends and family to other sources that are outside my scope of } \\
\text { practice. }\end{array}$ & 3.83 & 0.4290 \\
\hline I abide by the Dietetics Code of Ethics. & 0.63 & 0.8900 \\
\hline $\begin{array}{l}\text { I use nutrition information to diagnose health conditions in family } \\
\text { members and individuals. }\end{array}$ & 3.55 & 0.4699 \\
\hline Major & 0.52 & 0.4705 \\
\hline
\end{tabular}


Table 41 (Part A): UNDERWEIGHT: Eat a high protein, low carbohydrate diet.

\begin{tabular}{|c|c|c|c|c|c|c|c|}
\hline \multicolumn{8}{|c|}{ UNDERWEIGHT: Eat a high protein, low carbohydrate diet. } \\
\hline \multirow{5}{*}{$\begin{array}{l}\text { Parameter } \\
\text { I only deliver } \\
\text { information allowed } \\
\text { at the student level } \\
\text { by the CDR, as } \\
\text { specified in the } \\
\text { Dietetic Scope of } \\
\text { Practice. }\end{array}$} & \multirow{2}{*}{$\begin{array}{l}\text { Response } \\
\text { Rarely }\end{array}$} & \multirow{2}{*}{$\begin{array}{r}\text { Estimate } \\
0.1709\end{array}$} & \multirow{2}{*}{$\begin{array}{l}\text { Standard } \\
\text { Error } \\
2.0453\end{array}$} & \multicolumn{2}{|c|}{$\begin{array}{l}\text { Wald 95\% } \\
\text { Confidence Limits }\end{array}$} & \multirow{2}{*}{$\begin{array}{l}\text { Wald } \\
\text { Chi-Square } \\
0.01\end{array}$} & \multirow{2}{*}{ 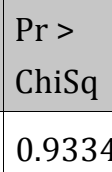 } \\
\hline & & & & -3.8378 & 4.1796 & & \\
\hline & Sometimes & -0.4641 & 0.8545 & -2.1389 & 1.2107 & 0.30 & 0.5870 \\
\hline & Often & -1.0957 & 0.8582 & -2.7777 & 0.5862 & 1.63 & 0.2017 \\
\hline & Always & 0.0000 & 0.0000 & 0.0000 & 0.0000 & & \\
\hline \multirow{5}{*}{$\begin{array}{l}\text { I comprehend the } \\
\text { scope of practice. }\end{array}$} & Never & -0.4742 & 1.7564 & -3.9168 & 2.9683 & 0.07 & 0.7872 \\
\hline & Rarely & -1.2114 & 1.4358 & -4.0255 & 1.6028 & 0.71 & 0.3988 \\
\hline & Sometimes & -0.8508 & 0.8678 & -2.5517 & 0.8501 & 0.96 & 0.3269 \\
\hline & Often & 0.4392 & 0.7785 & -1.0867 & 1.9651 & 0.32 & 0.5727 \\
\hline & Always & 0.0000 & 0.0000 & 0.0000 & 0.0000 & & \\
\hline \multirow{5}{*}{$\begin{array}{l}\text { I refer friends and } \\
\text { family to other } \\
\text { sources that are } \\
\text { outside my scope of } \\
\text { practice. }\end{array}$} & Never & -22.3973 & 85564.95 & -167727 & 167681.8 & 0.00 & 0.9998 \\
\hline & Rarely & 1.7498 & 1.9222 & -2.0177 & 5.5172 & 0.83 & 0.3627 \\
\hline & Sometimes & 0.8596 & 0.7014 & -0.5151 & 2.2343 & 1.50 & 0.2204 \\
\hline & Often & 0.7293 & 0.6162 & -0.4783 & 1.9369 & 1.40 & 0.2365 \\
\hline & Always & 0.0000 & 0.0000 & 0.0000 & 0.0000 & & \\
\hline \multirow{4}{*}{$\begin{array}{l}\text { I abide by the } \\
\text { Dietetics Code of } \\
\text { Ethics. }\end{array}$} & Rarely & 2.6110 & 2.1828 & -1.6673 & 6.8893 & 1.43 & 0.2316 \\
\hline & Sometimes & 0.6361 & 0.7780 & -0.8887 & 2.1610 & 0.67 & 0.4136 \\
\hline & Often & 0.2332 & 0.6101 & -0.9626 & 1.4290 & 0.15 & 0.7023 \\
\hline & Always & 0.0000 & 0.0000 & 0.0000 & 0.0000 & & \\
\hline \multirow{5}{*}{$\begin{array}{l}\text { I use nutrition } \\
\text { information to } \\
\text { diagnose health } \\
\text { conditions in family } \\
\text { members and } \\
\text { individuals. }\end{array}$} & Never & -2.4851 & 1.1405 & -4.7204 & -0.2497 & 4.75 & 0.0293 \\
\hline & Rarely & -2.4707 & 0.9810 & -4.3935 & -0.5480 & 6.34 & 0.0118 \\
\hline & Sometimes & -1.5843 & 0.9496 & -3.4455 & 0.2770 & 2.78 & 0.0953 \\
\hline & Often & -1.3093 & 1.0084 & -3.2858 & 0.6672 & 1.69 & 0.1942 \\
\hline & Always & 0.0000 & 0.0000 & 0.0000 & 0.0000 & & \\
\hline \multirow{2}{*}{ Major } & Dietetics & -0.1658 & 0.5261 & -1.1969 & 0.8652 & 0.10 & 0.7526 \\
\hline & Human Ntr & 0.0000 & 0.0000 & 0.0000 & 0.0000 & & \\
\hline
\end{tabular}


Table 41 (Part B): UNDERWEIGHT: Eat a high protein, low carbohydrate diet.

\begin{tabular}{|l|r|r|}
\hline \multicolumn{3}{|c|}{ UNDERWEIGHT: Eat a high protein, low carbohydrate diet. } \\
\hline Source & Chi-Square & Pr > ChiSq \\
\hline $\begin{array}{l}\text { I only deliver information allowed at the student level by the CDR, as } \\
\text { specified in the Dietetic Scope of Practice. }\end{array}$ & 2.25 & 0.5217 \\
\hline I comprehend the scope of practice. & 5.70 & 0.2227 \\
\hline $\begin{array}{l}\text { I refer friends and family to other sources that are outside my scope of } \\
\text { practice. }\end{array}$ & 3.63 & 0.4579 \\
\hline I abide by the Dietetics Code of Ethics. & 1.94 & 0.5845 \\
\hline $\begin{array}{l}\text { I use nutrition information to diagnose health conditions in family } \\
\text { members and individuals. }\end{array}$ & 9.73 & 0.0452 \\
\hline Major & 0.10 & 0.7528 \\
\hline
\end{tabular}


Table 42 (Part A): UNDERWEIGHT: Get at least 60 minutes of exercise per day, most days of the week.

\begin{tabular}{|c|c|c|c|c|c|c|c|}
\hline \multicolumn{8}{|c|}{ UNDERWEIGHT: Get at least 60 minutes of exercise per day, most days of the week. } \\
\hline \multirow{5}{*}{$\begin{array}{l}\text { Parameter } \\
\text { I only deliver } \\
\text { information } \\
\text { allowed at the } \\
\text { student level by the } \\
\text { CDR, as specified in } \\
\text { the Dietetic Scope } \\
\text { of Practice. }\end{array}$} & \multirow{2}{*}{\begin{tabular}{|l} 
Response \\
Rarely
\end{tabular}} & \multirow{2}{*}{$\begin{array}{r}\text { Estimate } \\
2.3073\end{array}$} & \multirow{2}{*}{\begin{tabular}{|l}
$\begin{array}{l}\text { Standard } \\
\text { Error }\end{array}$ \\
1.9565
\end{tabular}} & \multicolumn{2}{|c|}{$\begin{array}{l}\text { Wald 95\% } \\
\text { Confidence Limits }\end{array}$} & \multirow{2}{*}{\begin{tabular}{|r} 
Wald \\
Chi-Square \\
1.39
\end{tabular}} & \multirow{2}{*}{$\begin{array}{l}\begin{array}{l}\mathrm{Pr}> \\
\mathrm{ChiSq}\end{array} \\
0.2383\end{array}$} \\
\hline & & & & -1.5274 & 6.1420 & & \\
\hline & Sometimes & -0.1551 & 0.8770 & -1.8740 & 1.5638 & 0.03 & 0.8596 \\
\hline & Often & -0.2048 & 0.9043 & -1.9772 & 1.5675 & 0.05 & 0.8208 \\
\hline & Always & 0.0000 & 0.0000 & 0.0000 & 0.0000 & & \\
\hline \multirow{5}{*}{$\begin{array}{l}\text { I comprehend the } \\
\text { scope of practice. }\end{array}$} & Never & -28.2407 & 92265.91 & -180866 & 180809.6 & 0.00 & 0.9998 \\
\hline & Rarely & -1.5403 & 1.3671 & -4.2198 & 1.1391 & 1.27 & 0.2599 \\
\hline & Sometimes & -0.8195 & 0.8516 & -2.4886 & 0.8496 & 0.93 & 0.3359 \\
\hline & Often & -0.4790 & 0.7727 & -1.9935 & 1.0354 & 0.38 & 0.5353 \\
\hline & Always & 0.0000 & 0.0000 & 0.0000 & 0.0000 & & \\
\hline \multirow{5}{*}{$\begin{array}{l}\text { I refer friends and } \\
\text { family to other } \\
\text { sources that are } \\
\text { outside my scope of } \\
\text { practice. }\end{array}$} & Never & -0.2998 & 1.9382 & -4.0987 & 3.4990 & 0.02 & 0.8771 \\
\hline & Rarely & -3.9171 & 1.7909 & -7.4272 & -0.4071 & 4.78 & 0.0287 \\
\hline & Sometimes & -0.3539 & 0.7159 & -1.7570 & 1.0491 & 0.24 & 0.6210 \\
\hline & Often & -0.7295 & 0.5957 & -1.8971 & 0.4380 & 1.50 & 0.2207 \\
\hline & Always & 0.0000 & 0.0000 & 0.0000 & 0.0000 & & \\
\hline \multirow{4}{*}{$\begin{array}{l}\text { I abide by the } \\
\text { Dietetics Code of } \\
\text { Ethics. }\end{array}$} & Rarely & -2.7436 & 2.0124 & -6.6879 & 1.2007 & 1.86 & 0.1728 \\
\hline & Sometimes & -0.1731 & 0.8216 & -1.7833 & 1.4372 & 0.04 & 0.8332 \\
\hline & Often & -0.6468 & 0.7108 & -2.0400 & 0.7463 & 0.83 & 0.3628 \\
\hline & Always & 0.0000 & 0.0000 & 0.0000 & 0.0000 & & \\
\hline \multirow{5}{*}{$\begin{array}{l}\text { I use nutrition } \\
\text { information to } \\
\text { diagnose health } \\
\text { conditions in family } \\
\text { members and } \\
\text { individuals. }\end{array}$} & Never & -1.7681 & 1.0153 & -3.7580 & 0.2218 & 3.03 & 0.0816 \\
\hline & Rarely & -0.8128 & 0.8182 & -2.4164 & 0.7908 & 0.99 & 0.3205 \\
\hline & Sometimes & -1.5846 & 0.8384 & -3.2278 & 0.0586 & 3.57 & 0.0588 \\
\hline & Often & -0.9366 & 0.8779 & -2.6573 & 0.7841 & 1.14 & 0.2860 \\
\hline & Always & 0.0000 & 0.0000 & 0.0000 & 0.0000 & & \\
\hline \multirow{2}{*}{ Major } & Dietetics & -0.5711 & 0.5144 & -1.5793 & 0.4372 & 1.23 & 0.2669 \\
\hline & Human Ntr & 0.0000 & 0.0000 & 0.0000 & 0.0000 & & \\
\hline
\end{tabular}


Table 42 (Part B): UNDERWEIGHT: Get at least 60 minutes of exercise per day, most days of the week.

\begin{tabular}{|l|r|r|}
\hline \multicolumn{2}{|c|}{ UNDERWEIGHT: Get at least 60 minutes of exercise per day, most days of the week. } \\
\hline Source & Chi-Square & Pr > ChiSq \\
\hline $\begin{array}{l}\text { I only deliver information allowed at the student level by the CDR, as } \\
\text { specified in the Dietetic Scope of Practice. }\end{array}$ & 1.83 & 0.6080 \\
\hline I comprehend the scope of practice. & 10.10 & 0.0387 \\
\hline $\begin{array}{l}\text { I refer friends and family to other sources that are outside my scope of } \\
\text { practice. }\end{array}$ & 5.71 & 0.2218 \\
\hline $\begin{array}{l}\text { I abide by the Dietetics Code of Ethics. } \\
\text { I use nutrition information to diagnose health conditions in family } \\
\text { members and individuals. }\end{array}$ & 3.21 & 0.3610 \\
\hline Major & 5.39 & 0.2494 \\
\hline
\end{tabular}


Table 43 (Part A): UNDERWEIGHT: Eat several smaller meals throughout the day instead of 3 large meals.

\begin{tabular}{|c|c|c|c|c|c|c|c|}
\hline \multicolumn{8}{|c|}{ UNDERWEIGHT: Eat several smaller meals throughout the day instead of 3 large meals. } \\
\hline \multirow{5}{*}{$\begin{array}{l}\text { Parameter } \\
\text { I only deliver } \\
\text { information } \\
\text { allowed at the } \\
\text { student level by the } \\
\text { CDR, as specified in } \\
\text { the Dietetic Scope } \\
\text { of Practice. }\end{array}$} & \multirow{2}{*}{\begin{tabular}{|l} 
Response \\
Rarely
\end{tabular}} & \multirow{2}{*}{$\begin{array}{r}\text { Estimate } \\
-5.1165\end{array}$} & \multirow{2}{*}{$\begin{array}{l}\text { Standard } \\
\text { Error } \\
2.2067\end{array}$} & \multicolumn{2}{|c|}{$\begin{array}{l}\text { Wald 95\% } \\
\text { Confidence Limits }\end{array}$} & \multirow{2}{*}{\begin{tabular}{|r}
$\begin{array}{l}\text { Wald } \\
\text { Chi-Square }\end{array}$ \\
5.38
\end{tabular}} & \multirow{2}{*}{ 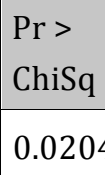 } \\
\hline & & & & -9.4416 & -0.7915 & & \\
\hline & Sometimes & -0.0150 & 0.8519 & -1.6848 & 1.6547 & 0.00 & 0.9859 \\
\hline & Often & -0.3099 & 0.8768 & -2.0283 & 1.4085 & 0.12 & 0.7238 \\
\hline & Always & 0.0000 & 0.0000 & 0.0000 & 0.0000 & & \\
\hline \multirow{5}{*}{$\begin{array}{l}\text { I comprehend the } \\
\text { scope of practice. }\end{array}$} & Never & 25.0375 & 138138.3 & -270721 & 270771.2 & 0.00 & 0.9999 \\
\hline & Rarely & 1.5383 & 1.5401 & -1.4803 & 4.5569 & 1.00 & 0.3179 \\
\hline & Sometimes & 1.4588 & 0.8312 & -0.1704 & 3.0880 & 3.08 & 0.0793 \\
\hline & Often & 0.8163 & 0.7377 & -0.6296 & 2.2621 & 1.22 & 0.2685 \\
\hline & Always & 0.0000 & 0.0000 & 0.0000 & 0.0000 & & \\
\hline \multirow{5}{*}{$\begin{array}{l}\text { I refer friends and } \\
\text { family to other } \\
\text { sources that are } \\
\text { outside my scope of } \\
\text { practice. }\end{array}$} & Never & 26.2124 & 138138.3 & -270720 & 270772.4 & 0.00 & 0.9998 \\
\hline & Rarely & -3.7842 & 1.7178 & -7.1511 & -0.4173 & 4.85 & 0.0276 \\
\hline & Sometimes & -0.2460 & 0.6740 & -1.5669 & 1.0750 & 0.13 & 0.7151 \\
\hline & Often & -0.7681 & 0.6012 & -1.9465 & 0.4102 & 1.63 & 0.2014 \\
\hline & Always & 0.0000 & 0.0000 & 0.0000 & 0.0000 & & \\
\hline \multirow{4}{*}{$\begin{array}{l}\text { I abide by the } \\
\text { Dietetics Code of } \\
\text { Ethics. }\end{array}$} & Rarely & 4.9884 & 2.2859 & 0.5080 & 9.4688 & 4.76 & 0.0291 \\
\hline & Sometimes & -1.2331 & 0.7850 & -2.7717 & 0.3056 & 2.47 & 0.1162 \\
\hline & Often & -1.2991 & 0.6409 & -2.5552 & -0.0430 & 4.11 & 0.0427 \\
\hline & Always & 0.0000 & 0.0000 & 0.0000 & 0.0000 & & \\
\hline \multirow{5}{*}{$\begin{array}{l}\text { I use nutrition } \\
\text { information to } \\
\text { diagnose health } \\
\text { conditions in family } \\
\text { members and } \\
\text { individuals. }\end{array}$} & Never & -1.6708 & 0.9866 & -3.6045 & 0.2629 & 2.87 & 0.0904 \\
\hline & Rarely & -0.6253 & 0.7995 & -2.1923 & 0.9417 & 0.61 & 0.4341 \\
\hline & Sometimes & -0.5054 & 0.7753 & -2.0249 & 1.0141 & 0.42 & 0.5145 \\
\hline & Often & -0.6223 & 0.8554 & -2.2989 & 1.0543 & 0.53 & 0.4669 \\
\hline & Always & 0.0000 & 0.0000 & 0.0000 & 0.0000 & & \\
\hline \multirow{2}{*}{ Major } & Dietetics & 0.1456 & 0.4933 & -0.8212 & 1.1124 & 0.09 & 0.7678 \\
\hline & Human Ntr & 0.0000 & 0.0000 & 0.0000 & 0.0000 & & \\
\hline
\end{tabular}


Table 43 (Part B): UNDERWEIGHT: Eat several smaller meals throughout the day instead of 3 large meals.

UNDERWEIGHT: Eat several smaller meals throughout the day instead of 3 large meals.

\begin{tabular}{|l|r|r|}
\hline Source & Chi-Square & Pr > ChiSq \\
\hline $\begin{array}{l}\text { I only deliver information allowed at the student level by the CDR, as } \\
\text { specified in the Dietetic Scope of Practice. }\end{array}$ & 6.64 & 0.0844 \\
\hline I comprehend the scope of practice. & 5.38 & 0.2506 \\
\hline $\begin{array}{l}\text { I refer friends and family to other sources that are outside my scope of } \\
\text { practice. }\end{array}$ & 11.61 & 0.0205 \\
\hline I abide by the Dietetics Code of Ethics. & 9.84 & 0.0200 \\
\hline $\begin{array}{l}\text { I use nutrition information to diagnose health conditions in family } \\
\text { members and individuals. }\end{array}$ & 3.08 & 0.5442 \\
\hline Major & 0.09 & 0.7679 \\
\hline
\end{tabular}


Table 44 (Part A): UNDERWEIGHT: Avoid eating or drinking anything after dinner.

\begin{tabular}{|c|c|c|c|c|c|c|c|}
\hline \multicolumn{8}{|c|}{ UNDERWEIGHT: Avoid eating or drinking anything after dinner. } \\
\hline \multirow{5}{*}{$\begin{array}{l}\text { Parameter } \\
\text { I only deliver } \\
\text { information } \\
\text { allowed at the } \\
\text { student level by the } \\
\text { CDR, as specified in } \\
\text { the Dietetic Scope } \\
\text { of Practice. }\end{array}$} & \multirow{2}{*}{$\begin{array}{l}\text { Response } \\
\text { Rarely }\end{array}$} & \multirow{2}{*}{$\begin{array}{r}\text { Estimate } \\
3.1124\end{array}$} & \multirow{2}{*}{$\begin{array}{l}\begin{array}{l}\text { Standard } \\
\text { Error }\end{array} \\
1.9754\end{array}$} & \multicolumn{2}{|c|}{$\begin{array}{l}\text { Wald 95\% } \\
\text { Confidence Limits }\end{array}$} & \multirow{2}{*}{$\begin{array}{l}\text { Wald } \\
\text { Chi-Square } \\
2.48\end{array}$} & \multirow{2}{*}{\begin{tabular}{|l}
$\begin{array}{l}\mathrm{Pr}> \\
\text { ChiSq }\end{array}$ \\
0.1151
\end{tabular}} \\
\hline & & & & -0.7593 & 6.9841 & & \\
\hline & Sometimes & -0.9413 & 0.8487 & -2.6046 & 0.7220 & 1.23 & 0.2674 \\
\hline & Often & -1.5227 & 0.8714 & -3.2306 & 0.1852 & 3.05 & 0.0806 \\
\hline & Always & 0.0000 & 0.0000 & 0.0000 & 0.0000 & & \\
\hline \multirow{5}{*}{$\begin{array}{l}\text { I comprehend the } \\
\text { scope of practice. }\end{array}$} & Never & -24.5713 & 142765.8 & -279840 & 279791.2 & 0.00 & 0.9999 \\
\hline & Rarely & -1.9534 & 1.4456 & -4.7867 & 0.8798 & 1.83 & 0.1766 \\
\hline & Sometimes & 1.0978 & 0.8404 & -0.5494 & 2.7450 & 1.71 & 0.1915 \\
\hline & Often & 0.8469 & 0.7684 & -0.6592 & 2.3530 & 1.21 & 0.2704 \\
\hline & Always & 0.0000 & 0.0000 & 0.0000 & 0.0000 & & \\
\hline \multirow{5}{*}{$\begin{array}{l}\text { I refer friends and } \\
\text { family to other } \\
\text { sources that are } \\
\text { outside my scope } \\
\text { of practice. }\end{array}$} & Never & -23.4128 & 142765.8 & -279839 & 279792.4 & 0.00 & 0.9999 \\
\hline & Rarely & 0.5985 & 1.6632 & -2.6612 & 3.8582 & 0.13 & 0.7189 \\
\hline & Sometimes & 0.4431 & 0.6818 & -0.8932 & 1.7793 & 0.42 & 0.5158 \\
\hline & Often & 0.6789 & 0.6129 & -0.5224 & 1.8802 & 1.23 & 0.2680 \\
\hline & Always & 0.0000 & 0.0000 & 0.0000 & 0.0000 & & \\
\hline \multirow{4}{*}{$\begin{array}{l}\text { I abide by the } \\
\text { Dietetics Code of } \\
\text { Ethics. }\end{array}$} & Rarely & -2.2649 & 2.3308 & -6.8332 & 2.3034 & 0.94 & 0.3312 \\
\hline & Sometimes & 0.5138 & 0.7391 & -0.9348 & 1.9624 & 0.48 & 0.4869 \\
\hline & Often & 0.0734 & 0.5850 & -1.0732 & 1.2200 & 0.02 & 0.9002 \\
\hline & Always & 0.0000 & 0.0000 & 0.0000 & 0.0000 & & \\
\hline \multirow{5}{*}{$\begin{array}{l}\text { I use nutrition } \\
\text { information to } \\
\text { diagnose health } \\
\text { conditions in } \\
\text { family members } \\
\text { and individuals. }\end{array}$} & Never & -1.6608 & 1.1246 & -3.8650 & 0.5435 & 2.18 & 0.1397 \\
\hline & Rarely & -1.5066 & 0.8701 & -3.2119 & 0.1987 & 3.00 & 0.0833 \\
\hline & Sometimes & -0.8422 & 0.8435 & -2.4955 & 0.8110 & 1.00 & 0.3181 \\
\hline & Often & 0.0908 & 0.8987 & -1.6707 & 1.8522 & 0.01 & 0.9196 \\
\hline & Always & 0.0000 & 0.0000 & 0.0000 & 0.0000 & & \\
\hline \multirow{2}{*}{ Major } & Dietetics & 0.7889 & 0.5269 & -0.2438 & 1.8216 & 2.24 & 0.1343 \\
\hline & Human Ntr & 0.0000 & 0.0000 & 0.0000 & 0.0000 & & \\
\hline
\end{tabular}


Table 44 (Part B): UNDERWEIGHT: Avoid eating or drinking anything after dinner.

\begin{tabular}{|l|r|r|}
\hline \multicolumn{3}{|c|}{ UNDERWEIGHT: Avoid eating or drinking anything after dinner. } \\
\hline Source & Chi-Square & Pr > ChiSq \\
\hline $\begin{array}{l}\text { I only deliver information allowed at the student level by the CDR, as } \\
\text { specified in the Dietetic Scope of Practice. }\end{array}$ & 7.97 & 0.0467 \\
\hline $\begin{array}{l}\text { I comprehend the scope of practice. } \\
\text { I refer friends and family to other sources that are outside my scope of } \\
\text { practice. }\end{array}$ & 2.66 & 0.19 \\
\hline $\begin{array}{l}\text { I abide by the Dietetics Code of Ethics. } \\
\text { I use nutrition information to diagnose health conditions in family } \\
\text { members and individuals. }\end{array}$ & 1.82 & 0.0374 \\
\hline Major & 9.74 & 0.0450 \\
\hline
\end{tabular}


Table 45 (Part A): UNDERWEIGHT: Eat a high protein, high calorie diet.

\begin{tabular}{|c|c|c|c|c|c|c|c|}
\hline \multicolumn{8}{|c|}{ UNDERWEIGHT: Eat a high protein, high calorie diet. } \\
\hline \multirow{5}{*}{$\begin{array}{l}\text { Parameter } \\
\text { I only deliver } \\
\text { information } \\
\text { allowed at the } \\
\text { student level by the } \\
\text { CDR, as specified in } \\
\text { the Dietetic Scope } \\
\text { of Practice. }\end{array}$} & \multirow{2}{*}{$\begin{array}{l}\text { Response } \\
\text { Rarely }\end{array}$} & \multirow{2}{*}{\begin{tabular}{|r} 
Estimate \\
-2.7023
\end{tabular}} & \multirow{2}{*}{\begin{tabular}{|l|l}
$\begin{array}{l}\text { Standard } \\
\text { Error }\end{array}$ \\
2.1108
\end{tabular}} & \multicolumn{2}{|c|}{$\begin{array}{l}\text { Wald 95\% } \\
\text { Confidence Limits }\end{array}$} & \multirow{2}{*}{\begin{tabular}{|r}
$\begin{array}{l}\text { Wald } \\
\text { Chi-Square }\end{array}$ \\
1.64
\end{tabular}} & \multirow{2}{*}{ 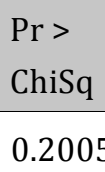 } \\
\hline & & & & -6.8395 & 1.4348 & & \\
\hline & Sometimes & 1.1066 & 0.8292 & -0.5187 & 2.7319 & 1.78 & 0.1820 \\
\hline & Often & 2.0929 & 0.8510 & 0.4250 & 3.7608 & 6.05 & 0.0139 \\
\hline & Always & 0.0000 & 0.0000 & 0.0000 & 0.0000 & & \\
\hline \multirow{5}{*}{$\begin{array}{l}\text { I comprehend the } \\
\text { scope of practice. }\end{array}$} & Never & 24.7504 & 144308.4 & -282814 & 282863.9 & 0.00 & 0.9999 \\
\hline & Rarely & 1.7458 & 1.5226 & -1.2385 & 4.7301 & 1.31 & 0.2516 \\
\hline & Sometimes & -1.2707 & 0.8620 & -2.9603 & 0.4189 & 2.17 & 0.1405 \\
\hline & Often & -1.2644 & 0.7625 & -2.7588 & 0.2301 & 2.75 & 0.0973 \\
\hline & Always & 0.0000 & 0.0000 & 0.0000 & 0.0000 & & \\
\hline \multirow{5}{*}{$\begin{array}{l}\text { I refer friends and } \\
\text { family to other } \\
\text { sources that are } \\
\text { outside my scope of } \\
\text { practice. }\end{array}$} & Never & 24.9923 & 144308.4 & -282814 & 282864.2 & 0.00 & 0.9999 \\
\hline & Rarely & 0.5608 & 1.7679 & -2.9043 & 4.0258 & 0.10 & 0.7511 \\
\hline & Sometimes & 0.5140 & 0.6902 & -0.8388 & 1.8668 & 0.55 & 0.4564 \\
\hline & Often & 0.0706 & 0.5636 & -1.0340 & 1.1752 & 0.02 & 0.9003 \\
\hline & Always & 0.0000 & 0.0000 & 0.0000 & 0.0000 & & \\
\hline \multirow{4}{*}{$\begin{array}{l}\text { I abide by the } \\
\text { Dietetics Code of } \\
\text { Ethics. }\end{array}$} & Rarely & 4.2047 & 2.5353 & -0.7645 & 9.1739 & 2.75 & 0.0972 \\
\hline & Sometimes & -0.7500 & 0.7789 & -2.2766 & 0.7766 & 0.93 & 0.3356 \\
\hline & Often & -0.4113 & 0.6279 & -1.6420 & 0.8195 & 0.43 & 0.5125 \\
\hline & Always & 0.0000 & 0.0000 & 0.0000 & 0.0000 & & \\
\hline \multirow{5}{*}{$\begin{array}{l}\text { I use nutrition } \\
\text { information to } \\
\text { diagnose health } \\
\text { conditions in family } \\
\text { members and } \\
\text { individuals. }\end{array}$} & Never & -0.5847 & 1.0539 & -2.6503 & 1.4808 & 0.31 & 0.5790 \\
\hline & Rarely & -1.1682 & 0.8943 & -2.9209 & 0.5845 & 1.71 & 0.1914 \\
\hline & Sometimes & -1.1029 & 0.8429 & -2.7549 & 0.5492 & 1.71 & 0.1907 \\
\hline & Often & -0.5010 & 0.9133 & -2.2911 & 1.2890 & 0.30 & 0.5833 \\
\hline & Always & 0.0000 & 0.0000 & 0.0000 & 0.0000 & & \\
\hline \multirow{2}{*}{ Major } & Dietetics & -0.8668 & 0.5204 & -1.8867 & 0.1531 & 2.77 & 0.0958 \\
\hline & Human Ntr & 0.0000 & 0.0000 & 0.0000 & 0.0000 & & \\
\hline
\end{tabular}


Table 45 (Part B): UNDERWEIGHT: Eat a high protein, high calorie diet.

\begin{tabular}{|l|r|r|}
\hline \multicolumn{3}{|c|}{ UNDERWEIGHT: Eat a high protein, high calorie diet. } \\
\hline Source & Chi-Square & Pr > ChiSq \\
\hline $\begin{array}{l}\text { I only deliver information allowed at the student level by the CDR, as } \\
\text { specified in the Dietetic Scope of Practice. }\end{array}$ & 10.17 & 0.0172 \\
\hline $\begin{array}{l}\text { I comprehend the scope of practice. } \\
\text { I refer friends and family to other sources that are outside my scope of } \\
\text { practice. }\end{array}$ & 3.11 & 0.46 \\
\hline $\begin{array}{l}\text { I abide by the Dietetics Code of Ethics. } \\
\text { I use nutrition information to diagnose health conditions in family } \\
\text { members and individuals. }\end{array}$ & 4.48 & 0.2144 \\
\hline Major & 2.15 & 0.5323 \\
\hline
\end{tabular}


Table 46 (Part A): UNDERWEIGHT: Avoid calorically dense foods and beverages.

\begin{tabular}{|c|c|c|c|c|c|c|c|}
\hline \multicolumn{8}{|c|}{ UNDERWEIGHT: Avoid calorically dense foods and beverages. } \\
\hline \multirow{5}{*}{$\begin{array}{l}\text { Parameter } \\
\text { I only deliver } \\
\text { information allowed } \\
\text { at the student level } \\
\text { by the CDR, as } \\
\text { specified in the } \\
\text { Dietetic Scope of } \\
\text { Practice. }\end{array}$} & \multirow{2}{*}{$\begin{array}{l}\text { Response } \\
\text { Rarely }\end{array}$} & \multirow{2}{*}{$\begin{array}{r}\text { Estimate } \\
3.0666\end{array}$} & \multirow{2}{*}{\begin{tabular}{|l}
$\begin{array}{l}\text { Standard } \\
\text { Error }\end{array}$ \\
1.9213
\end{tabular}} & \multicolumn{2}{|c|}{$\begin{array}{l}\text { Wald 95\% } \\
\text { Confidence Limits }\end{array}$} & \multirow{2}{*}{\begin{tabular}{|r|}
$\begin{array}{l}\text { Wald } \\
\text { Chi-Square }\end{array}$ \\
2.55
\end{tabular}} & \multirow{2}{*}{$\begin{array}{l}\begin{array}{l}\text { Pr }> \\
\text { ChiSq }\end{array} \\
0.1105\end{array}$} \\
\hline & & & & -0.6991 & 6.8323 & & \\
\hline & Sometimes & -0.6283 & 0.8580 & -2.3100 & 1.0533 & 0.54 & 0.4640 \\
\hline & Often & -1.0558 & 0.8627 & -2.7466 & 0.6350 & 1.50 & 0.2210 \\
\hline & Always & 0.0000 & 0.0000 & 0.0000 & 0.0000 & & \\
\hline \multirow{5}{*}{$\begin{array}{l}\text { I comprehend the } \\
\text { scope of practice. }\end{array}$} & Never & -24.5859 & 127511.2 & -249942 & 249892.8 & 0.00 & 0.9998 \\
\hline & Rarely & 0.8603 & 1.3090 & -1.7053 & 3.4259 & 0.43 & 0.5110 \\
\hline & Sometimes & 2.2046 & 0.8783 & 0.4832 & 3.9259 & 6.30 & 0.0121 \\
\hline & Often & 2.0042 & 0.7947 & 0.4466 & 3.5617 & 6.36 & 0.0117 \\
\hline & Always & 0.0000 & 0.0000 & 0.0000 & 0.0000 & & \\
\hline \multirow{5}{*}{$\begin{array}{l}\text { I refer friends and } \\
\text { family to other } \\
\text { sources that are } \\
\text { outside my scope of } \\
\text { practice. }\end{array}$} & Never & -26.6257 & 127511.2 & -249944 & 249890.8 & 0.00 & 0.9998 \\
\hline & Rarely & -1.2730 & 1.5593 & -4.3292 & 1.7831 & 0.67 & 0.4143 \\
\hline & Sometimes & -0.0565 & 0.6751 & -1.3797 & 1.2667 & 0.01 & 0.9333 \\
\hline & Often & -0.5906 & 0.5901 & -1.7471 & 0.5660 & 1.00 & 0.3169 \\
\hline & Always & 0.0000 & 0.0000 & 0.0000 & 0.0000 & & \\
\hline \multirow{4}{*}{$\begin{array}{l}\text { I abide by the } \\
\text { Dietetics Code of } \\
\text { Ethics. }\end{array}$} & Rarely & -2.6278 & 2.1339 & -6.8102 & 1.5545 & 1.52 & 0.2181 \\
\hline & Sometimes & -1.0840 & 0.7493 & -2.5525 & 0.3845 & 2.09 & 0.1480 \\
\hline & Often & -1.1483 & 0.5868 & -2.2983 & 0.0018 & 3.83 & 0.0504 \\
\hline & Always & 0.0000 & 0.0000 & 0.0000 & 0.0000 & & \\
\hline \multirow{5}{*}{$\begin{array}{l}\text { I use nutrition } \\
\text { information to } \\
\text { diagnose health } \\
\text { conditions in family } \\
\text { members and } \\
\text { individuals. }\end{array}$} & Never & 0.2230 & 0.9943 & -1.7258 & 2.1719 & 0.05 & 0.8225 \\
\hline & Rarely & -1.0819 & 0.8368 & -2.7221 & 0.5583 & 1.67 & 0.1961 \\
\hline & Sometimes & -0.7977 & 0.7836 & -2.3335 & 0.7380 & 1.04 & 0.3086 \\
\hline & Often & -0.0551 & 0.8553 & -1.7315 & 1.6214 & 0.00 & 0.9487 \\
\hline & Always & 0.0000 & 0.0000 & 0.0000 & 0.0000 & & \\
\hline \multirow{2}{*}{ Major } & Dietetics & 1.2888 & 0.5509 & 0.2091 & 2.3685 & 5.47 & 0.0193 \\
\hline & Human Ntr & 0.0000 & 0.0000 & 0.0000 & 0.0000 & & \\
\hline
\end{tabular}


Table 46 (Part B): UNDERWEIGHT: Avoid calorically dense foods and beverages.

\begin{tabular}{|l|r|r|}
\hline \multicolumn{3}{|c|}{ UNDERWEIGHT: Avoid calorically dense foods and beverages. } \\
\hline Source & Chi-Square & Pr > ChiSq \\
\hline $\begin{array}{l}\text { I only deliver information allowed at the student level by the CDR, as } \\
\text { specified in the Dietetic Scope of Practice. }\end{array}$ & 6.16 & 0.1041 \\
\hline I comprehend the scope of practice. & 13.17 & 0.0105 \\
\hline $\begin{array}{l}\text { I refer friends and family to other sources that are outside my scope of } \\
\text { practice. }\end{array}$ & 6.60 & 0.1586 \\
\hline I abide by the Dietetics Code of Ethics. & 4.82 & 0.1853 \\
\hline $\begin{array}{l}\text { I use nutrition information to diagnose health conditions in family } \\
\text { members and individuals. }\end{array}$ & 4.69 & 0.3205 \\
\hline Major & 5.70 & 0.0170 \\
\hline
\end{tabular}


Table 47 (Part A): UNDERWEIGHT: Consume less alcoholic beverages.

\begin{tabular}{|c|c|c|c|c|c|c|c|}
\hline \multicolumn{8}{|c|}{ UNDERWEIGHT: Consume less alcoholic beverages. } \\
\hline \multirow{5}{*}{$\begin{array}{l}\text { Parameter } \\
\text { I only deliver } \\
\text { information } \\
\text { allowed at the } \\
\text { student level by the } \\
\text { CDR, as specified in } \\
\text { the Dietetic Scope } \\
\text { of Practice. }\end{array}$} & \multirow{2}{*}{$\begin{array}{l}\text { Response } \\
\text { Rarely }\end{array}$} & \multirow{2}{*}{$\begin{array}{r}\text { Estimate } \\
0.5948\end{array}$} & \multirow{2}{*}{\begin{tabular}{|r|}
$\begin{array}{l}\text { Standard } \\
\text { Error }\end{array}$ \\
1.9155
\end{tabular}} & \multicolumn{2}{|c|}{$\begin{array}{l}\text { Wald } 95 \% \\
\text { Confidence Limits }\end{array}$} & \multirow{2}{*}{\begin{tabular}{|r}
$\begin{array}{l}\text { Wald } \\
\text { Chi-Square }\end{array}$ \\
0.10
\end{tabular}} & \multirow{2}{*}{ 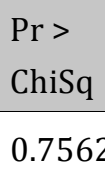 } \\
\hline & & & & -3.1594 & 4.3490 & & \\
\hline & Sometimes & -0.6213 & 0.8670 & -2.3206 & 1.0780 & 0.51 & 0.4736 \\
\hline & Often & -1.6042 & 0.8799 & -3.3288 & 0.1204 & 3.32 & 0.0683 \\
\hline & Always & 0.0000 & 0.0000 & 0.0000 & 0.0000 & & \\
\hline \multirow{5}{*}{$\begin{array}{l}\text { I comprehend the } \\
\text { scope of practice. }\end{array}$} & Never & 0.9246 & 1.8353 & -2.6726 & 4.5218 & 0.25 & 0.6144 \\
\hline & Rarely & 1.4999 & 1.2746 & -0.9984 & 3.9981 & 1.38 & 0.2393 \\
\hline & Sometimes & 2.6300 & 0.8605 & 0.9434 & 4.3166 & 9.34 & 0.0022 \\
\hline & Often & 2.1432 & 0.7604 & 0.6528 & 3.6336 & 7.94 & 0.0048 \\
\hline & Always & 0.0000 & 0.0000 & 0.0000 & 0.0000 & & \\
\hline \multirow{5}{*}{$\begin{array}{l}\text { I refer friends and } \\
\text { family to other } \\
\text { sources that are } \\
\text { outside my scope of } \\
\text { practice. }\end{array}$} & Never & -28.1823 & 97390.81 & -190911 & 190854.3 & 0.00 & 0.9998 \\
\hline & Rarely & 2.0895 & 1.5354 & -0.9198 & 5.0987 & 1.85 & 0.1735 \\
\hline & Sometimes & -0.0853 & 0.6659 & -1.3905 & 1.2198 & 0.02 & 0.8980 \\
\hline & Often & -0.6278 & 0.5786 & -1.7618 & 0.5062 & 1.18 & 0.2779 \\
\hline & Always & 0.0000 & 0.0000 & 0.0000 & 0.0000 & & \\
\hline \multirow{4}{*}{$\begin{array}{l}\text { I abide by the } \\
\text { Dietetics Code of } \\
\text { Ethics. }\end{array}$} & Rarely & -0.6919 & 2.1180 & -4.8432 & 3.4594 & 0.11 & 0.7439 \\
\hline & Sometimes & -1.3133 & 0.7774 & -2.8370 & 0.2105 & 2.85 & 0.0912 \\
\hline & Often & 0.2970 & 0.6118 & -0.9021 & 1.4962 & 0.24 & 0.6273 \\
\hline & Always & 0.0000 & 0.0000 & 0.0000 & 0.0000 & & \\
\hline \multirow{5}{*}{$\begin{array}{l}\text { I use nutrition } \\
\text { information to } \\
\text { diagnose health } \\
\text { conditions in family } \\
\text { members and } \\
\text { individuals. }\end{array}$} & Never & -1.0890 & 0.9569 & -2.9646 & 0.7865 & 1.30 & 0.2551 \\
\hline & Rarely & -1.5080 & 0.7858 & -3.0481 & 0.0320 & 3.68 & 0.0550 \\
\hline & Sometimes & -2.0130 & 0.7722 & -3.5266 & -0.4995 & 6.80 & 0.0091 \\
\hline & Often & -1.2365 & 0.8474 & -2.8974 & 0.4244 & 2.13 & 0.1445 \\
\hline & Always & 0.0000 & 0.0000 & 0.0000 & 0.0000 & & \\
\hline \multirow{2}{*}{ Major } & Dietetics & 1.7576 & 0.5437 & 0.6919 & 2.8232 & 10.45 & 0.0012 \\
\hline & Human Ntr & 0.0000 & 0.0000 & 0.0000 & 0.0000 & & \\
\hline
\end{tabular}


Table 47 (Part B): UNDERWEIGHT: Consume less alcoholic beverages.

\begin{tabular}{|l|r|r|}
\hline \multicolumn{3}{|c|}{ UNDERWEIGHT: Consume less alcoholic beverages. } \\
\hline Source & Chi-Square & Pr > ChiSq \\
\hline $\begin{array}{l}\text { I only deliver information allowed at the student level by the CDR, as } \\
\text { specified in the Dietetic Scope of Practice. }\end{array}$ & 5.27 & 0.1533 \\
\hline I comprehend the scope of practice. & 10.45 & 0.0334 \\
\hline $\begin{array}{l}\text { I refer friends and family to other sources that are outside my scope of } \\
\text { practice. }\end{array}$ & 13.37 & 0.0096 \\
\hline $\begin{array}{l}\text { I abide by the Dietetics Code of Ethics. } \\
\text { I use nutrition information to diagnose health conditions in family } \\
\text { members and individuals. }\end{array}$ & 6.84 & 0.0771 \\
\hline Major & 10.85 & 0.0999 \\
\hline
\end{tabular}


Table 48 (Part A): UNDERWEIGHT: Make smart food decisions when eating away from home.

\begin{tabular}{|c|c|c|c|c|c|c|c|}
\hline \multicolumn{8}{|c|}{ UNDERWEIGHT: Make smart food decisions when eating away from home. } \\
\hline \multirow{5}{*}{\begin{tabular}{|l} 
Parameter \\
I only deliver \\
information \\
allowed at the \\
student level by \\
the CDR, as \\
specified in the \\
Dietetic Scope of \\
Practice.
\end{tabular}} & \multirow{2}{*}{\begin{tabular}{|l} 
Response \\
Rarely
\end{tabular}} & \multirow{2}{*}{\begin{tabular}{|r|} 
Estimate \\
1.1128
\end{tabular}} & \multirow{2}{*}{\begin{tabular}{|l}
$\begin{array}{l}\text { Standard } \\
\text { Error }\end{array}$ \\
2.0624
\end{tabular}} & \multicolumn{2}{|c|}{$\begin{array}{l}\text { Wald 95\% } \\
\text { Confidence Limits }\end{array}$} & \multirow{2}{*}{\begin{tabular}{|l} 
Wald \\
Chi-Square \\
0.29
\end{tabular}} & \multirow{2}{*}{$\begin{array}{l}\begin{array}{l}\text { Pr }> \\
\text { ChiSq }\end{array} \\
0.5895\end{array}$} \\
\hline & & & & -2.9294 & 5.1550 & & \\
\hline & Sometimes & 0.2211 & 0.9733 & -1.6866 & 2.1288 & 0.05 & 0.8203 \\
\hline & Often & -1.0530 & 1.0063 & -3.0254 & 0.9193 & 1.09 & 0.2954 \\
\hline & Always & 0.0000 & 0.0000 & 0.0000 & 0.0000 & & \\
\hline \multirow{5}{*}{$\begin{array}{l}\text { I comprehend the } \\
\text { scope of practice. }\end{array}$} & Never & 22.4618 & 91520.43 & -179354 & 179399.2 & 0.00 & 0.9998 \\
\hline & Rarely & -1.4511 & 1.3377 & -4.0730 & 1.1707 & 1.18 & 0.2780 \\
\hline & Sometimes & -0.3936 & 0.9085 & -2.1743 & 1.3871 & 0.19 & 0.6649 \\
\hline & Often & -0.5737 & 0.8217 & -2.1843 & 1.0369 & 0.49 & 0.4851 \\
\hline & Always & 0.0000 & 0.0000 & 0.0000 & 0.0000 & & \\
\hline \multirow{5}{*}{$\begin{array}{l}\text { I refer friends and } \\
\text { family to other } \\
\text { sources that are } \\
\text { outside my scope } \\
\text { of practice. }\end{array}$} & Never & -1.9982 & 2.4122 & -6.7260 & 2.7297 & 0.69 & 0.4075 \\
\hline & Rarely & 0.1804 & 1.5549 & -2.8671 & 3.2280 & 0.01 & 0.9076 \\
\hline & Sometimes & -0.6953 & 0.7105 & -2.0879 & 0.6972 & 0.96 & 0.3277 \\
\hline & Often & -0.8146 & 0.6139 & -2.0178 & 0.3886 & 1.76 & 0.1845 \\
\hline & Always & 0.0000 & 0.0000 & 0.0000 & 0.0000 & & \\
\hline \multirow{4}{*}{$\begin{array}{l}\text { I abide by the } \\
\text { Dietetics Code of } \\
\text { Ethics. }\end{array}$} & Rarely & -0.2602 & 2.1846 & -4.5420 & 4.0216 & 0.01 & 0.9052 \\
\hline & Sometimes & -0.9279 & 0.8073 & -2.5102 & 0.6544 & 1.32 & 0.2504 \\
\hline & Often & -0.8147 & 0.6691 & -2.1262 & 0.4968 & 1.48 & 0.2234 \\
\hline & Always & 0.0000 & 0.0000 & 0.0000 & 0.0000 & & \\
\hline \multirow{5}{*}{$\begin{array}{l}\text { I use nutrition } \\
\text { information to } \\
\text { diagnose health } \\
\text { conditions in } \\
\text { family members } \\
\text { and individuals. }\end{array}$} & Never & -1.3215 & 1.0836 & -3.4452 & 0.8023 & 1.49 & 0.2226 \\
\hline & Rarely & -0.4547 & 0.8171 & -2.0562 & 1.1468 & 0.31 & 0.5779 \\
\hline & Sometimes & -0.7334 & 0.8205 & -2.3417 & 0.8748 & 0.80 & 0.3714 \\
\hline & Often & 0.0487 & 0.8669 & -1.6504 & 1.7477 & 0.00 & 0.9552 \\
\hline & Always & 0.0000 & 0.0000 & 0.0000 & 0.0000 & & \\
\hline \multirow{2}{*}{ Major } & Dietetics & 0.8875 & 0.5261 & -0.1437 & 1.9186 & 2.85 & 0.0916 \\
\hline & Human Ntr & 0.0000 & 0.0000 & 0.0000 & 0.0000 & & \\
\hline
\end{tabular}


Table 48 (Part B): UNDERWEIGHT: Make smart food decisions when eating away from home.

\begin{tabular}{|l|r|r|}
\hline \multicolumn{3}{|c|}{ UNDERWEIGHT: Make smart food decisions when eating away from home. } \\
\hline Source & Chi-Square & Pr > ChiSq \\
\hline $\begin{array}{l}\text { I only deliver information allowed at the student level by the CDR, as } \\
\text { specified in the Dietetic Scope of Practice. }\end{array}$ & 5.06 & 0.1674 \\
\hline I comprehend the scope of practice. & 2.60 & 0.6266 \\
\hline $\begin{array}{l}\text { I refer friends and family to other sources that are outside my scope of } \\
\text { practice. }\end{array}$ & 2.59 & 0.6288 \\
\hline $\begin{array}{l}\text { I abide by the Dietetics Code of Ethics. } \\
\text { I use nutrition information to diagnose health conditions in family } \\
\text { members and individuals. }\end{array}$ & 3.71 & 0.6345 \\
\hline Major & 2.89 & 0.4616 \\
\hline
\end{tabular}


Table 49 (Part A): UNDERWEIGHT: Use the Internet as a resource for nutrition facts at restaurants.

\begin{tabular}{|c|c|c|c|c|c|c|c|}
\hline \multicolumn{8}{|c|}{ UNDERWEIGHT: Use the Internet as a resource for nutrition facts at restaurants. } \\
\hline \multirow{5}{*}{$\begin{array}{l}\text { Parameter } \\
\text { I only deliver } \\
\text { information } \\
\text { allowed at the } \\
\text { student level by the } \\
\text { CDR, as specified in } \\
\text { the Dietetic Scope } \\
\text { of Practice. }\end{array}$} & \multirow{2}{*}{$\begin{array}{l}\text { Response } \\
\text { Rarely }\end{array}$} & \multirow{2}{*}{$\begin{array}{r}\text { Estimate } \\
-0.5468\end{array}$} & \multirow{2}{*}{$\begin{array}{l}\text { Standard } \\
\text { Error } \\
2.1009\end{array}$} & \multicolumn{2}{|c|}{$\begin{array}{l}\text { Wald 95\% } \\
\text { Confidence Limits }\end{array}$} & \multirow{2}{*}{$\begin{array}{l}\text { Wald } \\
\text { Chi-Square } \\
0.07\end{array}$} & \multirow{2}{*}{$\begin{array}{l}\text { Pr }> \\
\text { ChiSq }\end{array}$} \\
\hline & & & & -4.6645 & 3.5709 & & \\
\hline & Sometimes & -0.2589 & 0.9596 & -2.1397 & 1.6219 & 0.07 & 0.7873 \\
\hline & Often & -1.1045 & 0.9740 & -3.0135 & 0.8046 & 1.29 & 0.2568 \\
\hline & Always & 0.0000 & 0.0000 & 0.0000 & 0.0000 & & \\
\hline \multirow{5}{*}{$\begin{array}{l}\text { I comprehend the } \\
\text { scope of practice. }\end{array}$} & Never & 23.4466 & 141488.3 & -277289 & 277335.4 & 0.00 & 0.9999 \\
\hline & Rarely & -3.0763 & 1.4369 & -5.8926 & -0.2601 & 4.58 & 0.0323 \\
\hline & Sometimes & -1.3339 & 0.8702 & -3.0395 & 0.3717 & 2.35 & 0.1253 \\
\hline & Often & -0.4755 & 0.7825 & -2.0092 & 1.0583 & 0.37 & 0.5434 \\
\hline & Always & 0.0000 & 0.0000 & 0.0000 & 0.0000 & & \\
\hline \multirow{5}{*}{$\begin{array}{l}\text { I refer friends and } \\
\text { family to other } \\
\text { sources that are } \\
\text { outside my scope of } \\
\text { practice. }\end{array}$} & Never & 25.3280 & 141488.3 & -277287 & 277337.3 & 0.00 & 0.9999 \\
\hline & Rarely & -0.9840 & 2.1272 & -5.1534 & 3.1853 & 0.21 & 0.6437 \\
\hline & Sometimes & -0.4100 & 0.6816 & -1.7458 & 0.9258 & 0.36 & 0.5475 \\
\hline & Often & -0.3352 & 0.6132 & -1.5370 & 0.8666 & 0.30 & 0.5846 \\
\hline & Always & 0.0000 & 0.0000 & 0.0000 & 0.0000 & & \\
\hline \multirow{4}{*}{$\begin{array}{l}\text { I abide by the } \\
\text { Dietetics Code of } \\
\text { Ethics. }\end{array}$} & Rarely & 1.1133 & 2.2913 & -3.3776 & 5.6042 & 0.24 & 0.6271 \\
\hline & Sometimes & -0.1222 & 0.7866 & -1.6639 & 1.4196 & 0.02 & 0.8766 \\
\hline & Often & -0.8721 & 0.6642 & -2.1739 & 0.4298 & 1.72 & 0.1892 \\
\hline & Always & 0.0000 & 0.0000 & 0.0000 & 0.0000 & & \\
\hline \multirow{5}{*}{$\begin{array}{l}\text { I use nutrition } \\
\text { information to } \\
\text { diagnose health } \\
\text { conditions in family } \\
\text { members and } \\
\text { individuals. }\end{array}$} & Never & -1.3705 & 1.0571 & -3.4424 & 0.7014 & 1.68 & 0.1948 \\
\hline & Rarely & -1.0901 & 0.8282 & -2.7133 & 0.5331 & 1.73 & 0.1881 \\
\hline & Sometimes & -1.3471 & 0.8478 & -3.0088 & 0.3146 & 2.52 & 0.1121 \\
\hline & Often & -1.0137 & 0.8809 & -2.7403 & 0.7128 & 1.32 & 0.2498 \\
\hline & Always & 0.0000 & 0.0000 & 0.0000 & 0.0000 & & \\
\hline \multirow{2}{*}{ Major } & Dietetics & 0.9163 & 0.5130 & -0.0892 & 1.9218 & 3.19 & 0.0741 \\
\hline & Human Ntr & 0.0000 & 0.0000 & 0.0000 & 0.0000 & & \\
\hline
\end{tabular}


Table 49 (Part B): UNDERWEIGHT: Use the Internet as a resource for nutrition facts at restaurants.

\begin{tabular}{|l|r|r|}
\hline \multicolumn{2}{|c|}{ UNDERWEIGHT: Use the Internet as a resource for nutrition facts at restaurants. } \\
\hline Source & Chi-Square & Pr > ChiSq \\
\hline $\begin{array}{l}\text { I only deliver information allowed at the student level by the CDR, as } \\
\text { specified in the Dietetic Scope of Practice. }\end{array}$ & 2.84 & 0.4168 \\
\hline I comprehend the scope of practice. & 7.98 & 0.0923 \\
\hline $\begin{array}{l}\text { I refer friends and family to other sources that are outside my scope of } \\
\text { practice. }\end{array}$ & 4.07 & 0.3969 \\
\hline $\begin{array}{l}\text { I abide by the Dietetics Code of Ethics. } \\
\text { I use nutrition information to diagnose health conditions in family } \\
\text { members and individuals. }\end{array}$ & 2.97 & 0.3968 \\
\hline Major & 2.99 & 0.5587 \\
\hline
\end{tabular}


Table 50 (Part A): END RELATIONSHIP: Call a close family member or friend and talk about it.

\begin{tabular}{|c|c|c|c|c|c|c|c|}
\hline \multicolumn{8}{|c|}{ END RELATIONSHIP: Call a close family member or friend and talk about it. } \\
\hline \multirow{5}{*}{$\begin{array}{l}\text { Parameter } \\
\text { I only deliver } \\
\text { information } \\
\text { allowed at the } \\
\text { student level by the } \\
\text { CDR, as specified in } \\
\text { the Dietetic Scope } \\
\text { of Practice. }\end{array}$} & \multirow{2}{*}{\begin{tabular}{|l|} 
Response \\
Rarely
\end{tabular}} & \multirow{2}{*}{$\begin{array}{r}\text { Estimate } \\
25.2970\end{array}$} & \multirow{2}{*}{$\begin{array}{l}\text { Standard } \\
\text { Error } \\
1.6338\end{array}$} & \multicolumn{2}{|c|}{$\begin{array}{l}\text { Wald 95\% } \\
\text { Confidence Limits }\end{array}$} & \multirow{2}{*}{\begin{tabular}{|r|}
$\begin{array}{l}\text { Wald } \\
\text { Chi-Square }\end{array}$ \\
239.73
\end{tabular}} & \multirow{2}{*}{\begin{tabular}{|l|}
$\begin{array}{l}\text { Pr }> \\
\text { ChiSq }\end{array}$ \\
$<.0001$ \\
\end{tabular}} \\
\hline & & & & 22.0947 & 28.4992 & & \\
\hline & Sometimes & 0.2574 & 0.8393 & -1.3876 & 1.9023 & 0.09 & 0.7591 \\
\hline & Often & 0.1786 & 0.8551 & -1.4974 & 1.8546 & 0.04 & 0.8346 \\
\hline & Always & 0.0000 & 0.0000 & 0.0000 & 0.0000 & & \\
\hline \multirow{5}{*}{$\begin{array}{l}\text { I comprehend the } \\
\text { scope of practice. }\end{array}$} & Never & 25.4168 & 150965.2 & -295861 & 295911.9 & 0.00 & 0.9999 \\
\hline & Rarely & 0.4251 & 1.2754 & -2.0746 & 2.9248 & 0.11 & 0.7389 \\
\hline & Sometimes & 1.2362 & 0.8605 & -0.4503 & 2.9227 & 2.06 & 0.1508 \\
\hline & Often & 0.7724 & 0.7465 & -0.6907 & 2.2356 & 1.07 & 0.3008 \\
\hline & Always & 0.0000 & 0.0000 & 0.0000 & 0.0000 & & \\
\hline \multirow{5}{*}{$\begin{array}{l}\text { I refer friends and } \\
\text { family to other } \\
\text { sources that are } \\
\text { outside my scope } \\
\text { of practice. }\end{array}$} & Never & -2.6009 & 1.8388 & -6.2048 & 1.0030 & 2.00 & 0.1572 \\
\hline & Rarely & 0.7570 & 1.6330 & -2.4437 & 3.9576 & 0.21 & 0.6430 \\
\hline & Sometimes & -1.0746 & 0.7128 & -2.4716 & 0.3225 & 2.27 & 0.1317 \\
\hline & Often & -0.8499 & 0.5969 & -2.0198 & 0.3201 & 2.03 & 0.1545 \\
\hline & Always & 0.0000 & 0.0000 & 0.0000 & 0.0000 & & \\
\hline \multirow{4}{*}{$\begin{array}{l}\text { I abide by the } \\
\text { Dietetics Code of } \\
\text { Ethics. }\end{array}$} & Rarely & -27.5442 & 0.0000 & -27.5442 & -27.5442 & & \\
\hline & Sometimes & -0.2888 & 0.8041 & -1.8648 & 1.2871 & 0.13 & 0.7194 \\
\hline & Often & -0.0072 & 0.6348 & -1.2515 & 1.2371 & 0.00 & 0.9910 \\
\hline & Always & .0000 & 0.0000 & 0.0000 & 0.0000 & & \\
\hline \multirow{5}{*}{$\begin{array}{l}\text { I use nutrition } \\
\text { information to } \\
\text { diagnose health } \\
\text { conditions in } \\
\text { family members } \\
\text { and individuals. }\end{array}$} & Never & 0.7858 & 1.1178 & -1.4051 & 2.9767 & 0.49 & 0.4821 \\
\hline & Rarely & -1.3239 & 0.8561 & -3.0018 & 0.3541 & 2.39 & 0.1220 \\
\hline & Sometimes & -1.2646 & 0.8145 & -2.8610 & 0.3317 & 2.41 & 0.1205 \\
\hline & Often & -1.2110 & 0.9138 & -3.0019 & 0.5799 & 1.76 & 0.1851 \\
\hline & Always & 0.0000 & 0.0000 & 0.0000 & 0.0000 & & \\
\hline \multirow{2}{*}{ Major } & Dietetics & 0.3982 & 0.5266 & -0.6340 & 1.4303 & 0.57 & 0.4496 \\
\hline & Human Ntr & 0.0000 & 0.0000 & 0.0000 & 0.0000 & & \\
\hline
\end{tabular}


Table 50 (Part B): END RELATIONSHIP: Call a close family member or friend and talk about it.

\begin{tabular}{|l|r|r|}
\hline \multicolumn{3}{|c|}{ END RELATIONSHIP: Call a close family member or friend and talk about it. } \\
\hline Source & Chi-Square & $\mathrm{Pr}>$ ChiSq \\
\hline $\begin{array}{l}\text { I only deliver information allowed at the student level by the CDR, as } \\
\text { specified in the Dietetic Scope of Practice. }\end{array}$ & 3.99 & 0.2631 \\
\hline I comprehend the scope of practice. & 4.59 & 0.3318 \\
\hline $\begin{array}{l}\text { I refer friends and family to other sources that are outside my scope of } \\
\text { practice. }\end{array}$ & 4.91 & 0.2971 \\
\hline I abide by the Dietetics Code of Ethics. & 7.52 & 0.0571 \\
\hline $\begin{array}{l}\text { I use nutrition information to diagnose health conditions in family } \\
\text { members and individuals. }\end{array}$ & 8.23 & 0.0836 \\
\hline Major & 0.57 & 0.4501 \\
\hline
\end{tabular}


Table 51 (Part A): END RELATIONSHIP: Cry.

\begin{tabular}{|c|c|c|c|c|c|c|c|}
\hline \multicolumn{8}{|c|}{ END RELATIONSHIP: Cry. } \\
\hline \multirow{5}{*}{$\begin{array}{l}\text { Parameter } \\
\text { I only deliver } \\
\text { information } \\
\text { allowed at the } \\
\text { student level by the } \\
\text { CDR, as specified in } \\
\text { the Dietetic Scope } \\
\text { of Practice. }\end{array}$} & \multirow{2}{*}{$\begin{array}{l}\text { Response } \\
\text { Rarely }\end{array}$} & \multirow{2}{*}{\begin{tabular}{|c|} 
Estimate \\
21.7534
\end{tabular}} & \multirow{2}{*}{\begin{tabular}{|l}
$\begin{array}{l}\text { Standard } \\
\text { Error }\end{array}$ \\
1.7040
\end{tabular}} & \multicolumn{2}{|c|}{$\begin{array}{l}\text { Wald 95\% } \\
\text { Confidence Limits }\end{array}$} & \multirow{2}{*}{$\begin{array}{l}\text { Wald } \\
\text { Chi-Square } \\
162.97\end{array}$} & \multirow{2}{*}{$\begin{array}{l}\mathrm{Pr}> \\
\text { ChiSq } \\
<.000\end{array}$} \\
\hline & & & & 18.4137 & 25.0932 & & \\
\hline & Sometimes & -0.3363 & 0.9007 & -2.1016 & 1.4291 & 0.14 & 0.7089 \\
\hline & Often & -0.0758 & 0.9093 & -1.8579 & 1.7064 & 0.01 & 0.9336 \\
\hline & Always & 0.0000 & 0.0000 & 0.0000 & 0.0000 & & \\
\hline \multirow{5}{*}{$\begin{array}{l}\text { I comprehend the } \\
\text { scope of practice. }\end{array}$} & Never & 25.2729 & 145973.2 & -286077 & 286127.4 & 0.00 & 0.9999 \\
\hline & Rarely & 1.5456 & 1.4655 & -1.3267 & 4.4179 & 1.11 & 0.2916 \\
\hline & Sometimes & -0.0652 & 0.8565 & -1.7439 & 1.6134 & 0.01 & 0.9393 \\
\hline & Often & 0.2773 & 0.7395 & -1.1721 & 1.7267 & 0.14 & 0.7077 \\
\hline & Always & 0.0000 & 0.0000 & 0.0000 & 0.0000 & & \\
\hline \multirow{5}{*}{$\begin{array}{l}\text { I refer friends and } \\
\text { family to other } \\
\text { sources that are } \\
\text { outside my scope of } \\
\text { practice. }\end{array}$} & Never & -2.1300 & 1.8371 & -5.7306 & 1.4706 & 1.34 & 0.2463 \\
\hline & Rarely & -1.8740 & 1.6512 & -5.1103 & 1.3622 & 1.29 & 0.2564 \\
\hline & Sometimes & -0.9918 & 0.7175 & -2.3981 & 0.4145 & 1.91 & 0.1669 \\
\hline & Often & -1.0789 & 0.6201 & -2.2942 & 0.1365 & 3.03 & 0.0819 \\
\hline & Always & 0.0000 & 0.0000 & 0.0000 & 0.0000 & & \\
\hline \multirow{4}{*}{$\begin{array}{l}\text { I abide by the } \\
\text { Dietetics Code of } \\
\text { Ethics. }\end{array}$} & Rarely & -20.6085 & 0.0000 & -20.6085 & -20.6085 & & \\
\hline & Sometimes & 0.6518 & 0.7867 & -0.8900 & 2.1937 & 0.69 & 0.4073 \\
\hline & Often & -0.3240 & 0.6084 & -1.5165 & 0.8684 & 0.28 & 0.5943 \\
\hline & Always & 0.0000 & 0.0000 & 0.0000 & 0.0000 & & \\
\hline \multirow{5}{*}{$\begin{array}{l}\text { I use nutrition } \\
\text { information to } \\
\text { diagnose health } \\
\text { conditions in family } \\
\text { members and } \\
\text { individuals. }\end{array}$} & Never & 0.4001 & 1.2322 & -2.0149 & 2.8151 & 0.11 & 0.7454 \\
\hline & Rarely & 0.8210 & 1.0213 & -1.1807 & 2.8228 & 0.65 & 0.4215 \\
\hline & Sometimes & 0.0814 & 0.9816 & -1.8425 & 2.0053 & 0.01 & 0.9339 \\
\hline & Often & 1.0405 & 1.0777 & -1.0717 & 3.1526 & 0.93 & 0.3343 \\
\hline & Always & 0.0000 & 0.0000 & 0.0000 & 0.0000 & & \\
\hline \multirow{2}{*}{ Major } & Dietetics & 0.9604 & 0.5070 & -0.0333 & 1.9541 & 3.59 & 0.0582 \\
\hline & Human Ntr & 0.0000 & 0.0000 & 0.0000 & 0.0000 & & \\
\hline
\end{tabular}


Table 51 (Part B): END RELATIONSHIP: Cry.

\begin{tabular}{|l|r|r|}
\hline \multicolumn{3}{|c|}{ END RELATIONSHIP: Cry. } \\
\hline Source & Chi-Square & Pr > ChiSq \\
\hline $\begin{array}{l}\text { I only deliver information allowed at the student level by the CDR, as } \\
\text { specified in the Dietetic Scope of Practice. }\end{array}$ & 0.82 & 0.8443 \\
\hline $\begin{array}{l}\text { I comprehend the scope of practice. } \\
\text { I refer friends and family to other sources that are outside my scope of } \\
\text { practice. }\end{array}$ & 3.88 & 0.3000 \\
\hline $\begin{array}{l}\text { I abide by the Dietetics Code of Ethics. } \\
\text { I use nutrition information to diagnose health conditions in family } \\
\text { members and individuals. }\end{array}$ & 2.90 & 0.4186 \\
\hline Major & 3.93 & 0.4067 \\
\hline
\end{tabular}


Table 52 (Part A): END RELATIONSHIP: Go to the gym or exercise outdoors.

\begin{tabular}{|c|c|c|c|c|c|c|c|}
\hline \multicolumn{8}{|c|}{ END RELATIONSHIP: Go to the gym or exercise outdoors. } \\
\hline \multirow{5}{*}{$\begin{array}{l}\text { Parameter } \\
\text { I only deliver } \\
\text { information allowed } \\
\text { at the student level } \\
\text { by the CDR, as } \\
\text { specified in the } \\
\text { Dietetic Scope of } \\
\text { Practice. }\end{array}$} & \multirow{2}{*}{$\begin{array}{l}\text { Response } \\
\text { Rarely }\end{array}$} & \multirow{2}{*}{$\begin{array}{r}\text { Estimate } \\
0.6163\end{array}$} & \multirow{2}{*}{\begin{tabular}{|l}
$\begin{array}{l}\text { Standard } \\
\text { Error }\end{array}$ \\
1.8651
\end{tabular}} & \multicolumn{2}{|c|}{$\begin{array}{l}\text { Wald } 95 \% \\
\text { Confidence Limits }\end{array}$} & \multirow{2}{*}{\begin{tabular}{|r}
$\begin{array}{l}\text { Wald } \\
\text { Chi-Square }\end{array}$ \\
0.11
\end{tabular}} & \multirow{2}{*}{$\begin{array}{l}\text { Pr }> \\
\text { ChiSq }\end{array}$} \\
\hline & & & & -3.0392 & 4.2717 & & \\
\hline & Sometimes & 0.4223 & 0.8408 & -1.2257 & 2.0703 & 0.25 & 0.6155 \\
\hline & Often & 0.3996 & 0.8252 & -1.2178 & 2.0169 & 0.23 & 0.6282 \\
\hline & Always & 0.0000 & 0.0000 & 0.0000 & 0.0000 & & \\
\hline \multirow{5}{*}{$\begin{array}{l}\text { I comprehend the } \\
\text { scope of practice. }\end{array}$} & Never & 23.8824 & 83203.29 & -163052 & 163099.3 & 0.00 & 0.9998 \\
\hline & Rarely & 0.3662 & 1.3991 & -2.3760 & 3.1085 & 0.07 & 0.7935 \\
\hline & Sometimes & 0.2088 & 0.7563 & -1.2736 & 1.6912 & 0.08 & 0.7825 \\
\hline & Often & 0.0852 & 0.6872 & -1.2618 & 1.4322 & 0.02 & 0.9013 \\
\hline & Always & 0.0000 & 0.0000 & 0.0000 & 0.0000 & & \\
\hline \multirow{5}{*}{$\begin{array}{l}\text { I refer friends and } \\
\text { family to other } \\
\text { sources that are } \\
\text { outside my scope of } \\
\text { practice. }\end{array}$} & Never & -2.5883 & 1.9171 & -6.3459 & 1.1692 & 1.82 & 0.1770 \\
\hline & Rarely & -2.7748 & 2.1629 & -7.0141 & 1.4645 & 1.65 & 0.1995 \\
\hline & Sometimes & -1.3777 & 0.6648 & -2.6807 & -0.0747 & 4.29 & 0.0382 \\
\hline & Often & -0.9752 & 0.5774 & -2.1069 & 0.1565 & 2.85 & 0.0912 \\
\hline & Always & 0.0000 & 0.0000 & 0.0000 & 0.0000 & & \\
\hline \multirow{4}{*}{$\begin{array}{l}\text { I abide by the } \\
\text { Dietetics Code of } \\
\text { Ethics. }\end{array}$} & Rarely & 0.2545 & 1.9943 & -3.6543 & 4.1634 & 0.02 & 0.8984 \\
\hline & Sometimes & -0.5689 & 0.6972 & -1.9354 & 0.7976 & 0.67 & 0.4145 \\
\hline & Often & -0.4328 & 0.5630 & -1.5362 & 0.6706 & 0.59 & 0.4420 \\
\hline & Always & 0.0000 & 0.0000 & 0.0000 & 0.0000 & & \\
\hline \multirow{5}{*}{$\begin{array}{l}\text { I use nutrition } \\
\text { information to } \\
\text { diagnose health } \\
\text { conditions in family } \\
\text { members and } \\
\text { individuals. }\end{array}$} & Never & -0.4375 & 0.9959 & -2.3894 & 1.5143 & 0.19 & 0.6604 \\
\hline & Rarely & -1.1265 & 0.7683 & -2.6324 & 0.3794 & 2.15 & 0.1426 \\
\hline & Sometimes & -0.9599 & 0.7341 & -2.3988 & 0.4790 & 1.71 & 0.1910 \\
\hline & Often & -0.9464 & 0.8196 & -2.5529 & 0.6601 & 1.33 & 0.2482 \\
\hline & Always & 0.0000 & 0.0000 & 0.0000 & 0.0000 & & \\
\hline \multirow{2}{*}{ Major } & Dietetics & 0.6994 & 0.4873 & -0.2557 & 1.6545 & 2.06 & 0.1512 \\
\hline & Human Ntr & 0.0000 & 0.0000 & 0.0000 & 0.0000 & & \\
\hline
\end{tabular}


Table 52 (Part B): END RELATIONSHIP: Go to the gym or exercise outdoors.

\begin{tabular}{|l|r|r|}
\hline \multicolumn{3}{|c|}{ END RELATIONSHIP: Go to the gym or exercise outdoors. } \\
\hline Source & Chi-Square & Pr > ChiSq \\
\hline $\begin{array}{l}\text { I only deliver information allowed at the student level by the CDR, as } \\
\text { specified in the Dietetic Scope of Practice. }\end{array}$ & 0.30 & 0.9609 \\
\hline I comprehend the scope of practice. & 2.73 & 0.6043 \\
\hline $\begin{array}{l}\text { I refer friends and family to other sources that are outside my scope of } \\
\text { practice. }\end{array}$ & 6.37 & 0.1732 \\
\hline I abide by the Dietetics Code of Ethics. & 0.85 & 0.8364 \\
\hline $\begin{array}{l}\text { I use nutrition information to diagnose health conditions in family } \\
\text { members and individuals. }\end{array}$ & 2.52 & 0.6404 \\
\hline Major & 2.08 & 0.1494 \\
\hline
\end{tabular}


Table 53 (Part A): END RELATIONSHIP: Drink alcoholic beverages.

\begin{tabular}{|c|c|c|c|c|c|c|c|}
\hline \multicolumn{8}{|c|}{ END RELATIONSHIP: Drink alcoholic beverages. } \\
\hline \multirow{5}{*}{$\begin{array}{l}\text { Parameter } \\
\text { I only deliver } \\
\text { information allowed } \\
\text { at the student level by } \\
\text { the CDR, as specified } \\
\text { in the Dietetic Scope } \\
\text { of Practice. }\end{array}$} & \multirow{2}{*}{$\begin{array}{l}\text { Response } \\
\text { Rarely }\end{array}$} & \multirow{2}{*}{\begin{tabular}{|r} 
Estimate \\
3.4836
\end{tabular}} & \multirow{2}{*}{$\begin{array}{l}\begin{array}{l}\text { Standard } \\
\text { Error }\end{array} \\
1.9587\end{array}$} & \multicolumn{2}{|c|}{$\begin{array}{l}\text { Wald 95\% } \\
\text { Confidence Limits }\end{array}$} & \multirow{2}{*}{$\begin{array}{l}\begin{array}{l}\text { Wald } \\
\text { Chi-Square }\end{array} \\
3.16\end{array}$} & \multirow{2}{*}{$\begin{array}{l}\operatorname{Pr}> \\
\text { ChiSq }\end{array}$} \\
\hline & & & & -0.3554 & 7.3226 & & \\
\hline & Sometimes & -1.3706 & 0.8492 & -3.0351 & 0.2939 & 2.60 & 0.1065 \\
\hline & Often & -1.0349 & 0.8747 & -2.7493 & 0.6794 & 1.40 & 0.2367 \\
\hline & Always & 0.0000 & 0.0000 & 0.0000 & 0.0000 & & \\
\hline \multirow{5}{*}{$\begin{array}{l}\text { I comprehend the } \\
\text { scope of practice. }\end{array}$} & Never & 1.6120 & 1.7080 & -1.7356 & 4.9596 & 0.89 & 0.3453 \\
\hline & Rarely & -2.6564 & 1.5138 & -5.6234 & 0.3106 & 3.08 & 0.0793 \\
\hline & Sometimes & -0.2630 & 0.7531 & -1.7391 & 1.2132 & 0.12 & 0.7270 \\
\hline & Often & -0.2215 & 0.6853 & -1.5646 & 1.1216 & 0.10 & 0.7465 \\
\hline & Always & 0.0000 & 0.0000 & 0.0000 & 0.0000 & & \\
\hline \multirow{5}{*}{$\begin{array}{l}\text { I refer friends and } \\
\text { family to other } \\
\text { sources that are } \\
\text { outside my scope of } \\
\text { practice. }\end{array}$} & Never & 0.1309 & 1.7422 & -3.2838 & 3.5456 & 0.01 & 0.9401 \\
\hline & Rarely & 0.8115 & 1.6445 & -2.4116 & 4.0347 & 0.24 & 0.6217 \\
\hline & Sometimes & -0.3848 & 0.6619 & -1.6821 & 0.9125 & 0.34 & 0.5610 \\
\hline & Often & 0.2816 & 0.5565 & -0.8090 & 1.3723 & 0.26 & 0.6128 \\
\hline & Always & 0.0000 & 0.0000 & 0.0000 & 0.0000 & & \\
\hline \multirow{4}{*}{$\begin{array}{l}\text { I abide by the Dietetics } \\
\text { Code of Ethics. }\end{array}$} & Rarely & -3.8274 & 2.1715 & -8.0835 & 0.4287 & 3.11 & 0.0780 \\
\hline & Sometimes & 0.5451 & 0.7361 & -0.8976 & 1.9877 & 0.55 & 0.4590 \\
\hline & Often & 0.2209 & 0.5801 & -0.9161 & 1.3580 & 0.15 & 0.7033 \\
\hline & Always & 0.0000 & 0.0000 & 0.0000 & 0.0000 & & \\
\hline \multirow{5}{*}{$\begin{array}{l}\text { I use nutrition } \\
\text { information to } \\
\text { diagnose health } \\
\text { conditions in family } \\
\text { members and } \\
\text { individuals. }\end{array}$} & Never & -0.0364 & 0.9733 & -1.9441 & 1.8713 & 0.00 & 0.9702 \\
\hline & Rarely & 0.3161 & 0.8103 & -1.2721 & 1.9043 & 0.15 & 0.6965 \\
\hline & Sometimes & -0.0728 & 0.7957 & -1.6323 & 1.4867 & 0.01 & 0.9271 \\
\hline & Often & 0.1763 & 0.8847 & -1.5577 & 1.9104 & 0.04 & 0.8420 \\
\hline & Always & 0.0000 & 0.0000 & 0.0000 & 0.0000 & & \\
\hline \multirow{2}{*}{ Major } & Dietetics & -0.1315 & 0.5067 & -1.1246 & 0.8615 & 0.07 & 0.7952 \\
\hline & Human Ntr & 0.0000 & 0.0000 & 0.0000 & 0.0000 & & \\
\hline
\end{tabular}


Table 53 (Part B): END RELATIONSHIP: Drink alcoholic beverages.

\begin{tabular}{|l|r|r|}
\hline \multicolumn{3}{|c|}{ END RELATIONSHIP: Drink alcoholic beverages. } \\
\hline Source & Chi-Square & Pr > ChiSq \\
\hline $\begin{array}{l}\text { I only deliver information allowed at the student level by the CDR, as } \\
\text { specified in the Dietetic Scope of Practice. }\end{array}$ & 9.10 & 0.0280 \\
\hline I comprehend the scope of practice. & 5.17 & 0.2698 \\
\hline $\begin{array}{l}\text { I refer friends and family to other sources that are outside my scope of } \\
\text { practice. }\end{array}$ & 1.52 & 0.8232 \\
\hline $\begin{array}{l}\text { I abide by the Dietetics Code of Ethics. } \\
\text { I use nutrition information to diagnose health conditions in family } \\
\text { members and individuals. }\end{array}$ & 4.53 & 0.2098 \\
\hline Major & 0.61 & 0.9625 \\
\hline
\end{tabular}


Table 54 (Part A): END RELATIONSHIP: Immerse yourself in schoolwork.

\begin{tabular}{|c|c|c|c|c|c|c|c|}
\hline \multicolumn{8}{|c|}{ END RELATIONSHIP: Immerse yourself in your schoolwork. } \\
\hline \multirow{5}{*}{$\begin{array}{l}\text { Parameter } \\
\text { I only deliver } \\
\text { information } \\
\text { allowed at the } \\
\text { student level by the } \\
\text { CDR, as specified in } \\
\text { the Dietetic Scope } \\
\text { of Practice. }\end{array}$} & \multirow{2}{*}{\begin{tabular}{|l|} 
Response \\
Rarely
\end{tabular}} & \multirow{2}{*}{$\begin{array}{r}\text { Estimate } \\
-4.3774\end{array}$} & \multirow{2}{*}{\begin{tabular}{|r|}
$\begin{array}{l}\text { Standard } \\
\text { Error }\end{array}$ \\
1.9941
\end{tabular}} & \multicolumn{2}{|c|}{$\begin{array}{l}\text { Wald 95\% } \\
\text { Confidence Limits }\end{array}$} & \multirow{2}{*}{$\begin{array}{l}\text { Wald } \\
\text { Chi-Square } \\
4.82\end{array}$} & \multirow{2}{*}{\begin{tabular}{|l|}
$\begin{array}{l}\text { Pr }> \\
\text { ChiSq }\end{array}$ \\
0.0281
\end{tabular}} \\
\hline & & & & -8.2857 & -0.4691 & & \\
\hline & Sometimes & -1.2238 & 0.8762 & -2.9411 & 0.4935 & 1.95 & 0.1625 \\
\hline & Often & -1.6718 & 0.8771 & -3.3908 & 0.0472 & 3.63 & 0.056 \\
\hline & Always & 0.0000 & 0.0000 & 0.0000 & 0.0000 & & \\
\hline \multirow{5}{*}{$\begin{array}{l}\text { I comprehend the } \\
\text { scope of practice. }\end{array}$} & Never & 23.8930 & 67604.19 & -132478 & 132525.7 & 0.00 & 0.9997 \\
\hline & Rarely & 0.6697 & 1.4669 & -2.2054 & 3.5449 & 0.21 & 0.6480 \\
\hline & Sometimes & -1.4367 & 0.8060 & -3.0164 & 0.1429 & 3.18 & 0.074 \\
\hline & Often & -0.0168 & 0.7235 & -1.4348 & 1.4013 & 0.00 & 0.981 \\
\hline & Always & 0.0000 & 0.0000 & 0.0000 & 0.0000 & & \\
\hline \multirow{5}{*}{$\begin{array}{l}\text { I refer friends and } \\
\text { family to other } \\
\text { sources that are } \\
\text { outside my scope of } \\
\text { practice. }\end{array}$} & Never & 0.8645 & 1.7569 & -2.5790 & 4.3080 & 0.24 & 0.6227 \\
\hline & Rarely & 1.0825 & 3.5200 & -5.8165 & 7.9816 & 0.09 & 0.758 \\
\hline & Sometimes & -0.2629 & 0.6877 & -1.6108 & 1.0850 & 0.15 & 0.7023 \\
\hline & Often & 0.2628 & 0.6017 & -0.9165 & 1.4420 & 0.19 & 0.6623 \\
\hline & Always & 0.0000 & 0.0000 & 0.0000 & 0.0000 & & \\
\hline \multirow{4}{*}{$\begin{array}{l}\text { I abide by the } \\
\text { Dietetics Code of } \\
\text { Ethics. }\end{array}$} & Rarely & 6.5886 & 2.2008 & 2.2750 & 10.9021 & 8.96 & 0.0028 \\
\hline & Sometimes & -0.5243 & 0.7104 & -1.9167 & 0.8681 & 0.54 & 0.4605 \\
\hline & Often & 0.2888 & 0.5566 & -0.8021 & 1.3797 & 0.27 & 0.6039 \\
\hline & Always & 0.0000 & 0.0000 & 0.0000 & 0.0000 & & \\
\hline \multirow{5}{*}{$\begin{array}{l}\text { I use nutrition } \\
\text { information to } \\
\text { diagnose health } \\
\text { conditions in family } \\
\text { members and } \\
\text { individuals. }\end{array}$} & Never & -0.6502 & 0.9556 & -2.5232 & 1.2228 & 0.46 & 0.4963 \\
\hline & Rarely & 0.0284 & 0.7748 & -1.4902 & 1.5470 & 0.00 & 0.9708 \\
\hline & Sometimes & 0.8758 & 0.7874 & -0.6674 & 2.4190 & 1.24 & 0.2660 \\
\hline & Often & -0.3140 & 0.8551 & -1.9900 & 1.3620 & 0.13 & 0.7135 \\
\hline & Always & 0.0000 & 0.0000 & 0.0000 & 0.0000 & & \\
\hline \multirow{2}{*}{ Major } & Dietetics & -0.1584 & 0.5003 & -1.1390 & 0.8222 & 0.10 & 0.7516 \\
\hline & Human Ntr & 0.0000 & 0.0000 & 0.0000 & 0.0000 & & \\
\hline
\end{tabular}


Table 54 (Part B): END RELATIONSHIP: Immerse yourself in schoolwork.

END RELATIONSHIP: Immerse yourself in your schoolwork.

\begin{tabular}{|l|r|r|}
\hline Source & Chi-Square & Pr > ChiSq \\
\hline $\begin{array}{l}\text { I only deliver information allowed at the student level by the CDR, as } \\
\text { specified in the Dietetic Scope of Practice. }\end{array}$ & 7.08 & 0.0692 \\
\hline I comprehend the scope of practice. & 12.72 & 0.0127 \\
\hline $\begin{array}{l}\text { I refer friends and family to other sources that are outside my scope of } \\
\text { practice. }\end{array}$ & 1.17 & 0.8825 \\
\hline I abide by the Dietetics Code of Ethics. & 11.66 & 0.0087 \\
\hline $\begin{array}{l}\text { I use nutrition information to diagnose health conditions in family } \\
\text { members and individuals. }\end{array}$ & 6.41 & 0.1706 \\
\hline Major & 0.10 & 0.7516 \\
\hline
\end{tabular}


Table 55 (Part A): END RELATIONSHIP: Eat your favorite junk food.

\begin{tabular}{|c|c|c|c|c|c|c|c|}
\hline \multicolumn{8}{|c|}{ END RELATIONSHIP: Eat your favorite junk food. } \\
\hline \multirow{5}{*}{$\begin{array}{l}\text { Parameter } \\
\text { I only deliver } \\
\text { information allowed } \\
\text { at the student level by } \\
\text { the CDR, as specified } \\
\text { in the Dietetic Scope } \\
\text { of Practice. }\end{array}$} & \multirow{2}{*}{$\begin{array}{l}\text { Response } \\
\text { Rarely }\end{array}$} & \multirow{2}{*}{\begin{tabular}{|r|} 
Estimate \\
2.8243
\end{tabular}} & \multirow{2}{*}{\begin{tabular}{|l|l}
$\begin{array}{l}\text { Standard } \\
\text { Error }\end{array}$ \\
1.9103
\end{tabular}} & \multicolumn{2}{|c|}{$\begin{array}{l}\text { Wald 95\% } \\
\text { Confidence Limits }\end{array}$} & \multirow{2}{*}{$\begin{array}{l}\text { Wald } \\
\text { Chi-Square } \\
2.19\end{array}$} & \multirow{2}{*}{\begin{tabular}{|l|}
$\begin{array}{l}\text { Pr }> \\
\text { ChiSq }\end{array}$ \\
0.1393
\end{tabular}} \\
\hline & & & & -0.9197 & 6.5684 & & \\
\hline & Sometimes & -0.0026 & 0.8392 & -1.6474 & 1.6423 & 0.00 & 0.9976 \\
\hline & Often & 0.4975 & 0.8648 & -1.1975 & 2.1926 & 0.33 & 0.5651 \\
\hline & Always & 0.0000 & 0.0000 & 0.0000 & 0.0000 & & \\
\hline \multirow{5}{*}{$\begin{array}{l}\text { I comprehend the } \\
\text { scope of practice. }\end{array}$} & Never & 2.3208 & 1.8187 & -1.2438 & 5.8854 & 1.63 & 0.2019 \\
\hline & Rarely & -1.3123 & 1.3858 & -4.0284 & 1.4038 & 0.90 & 0.3437 \\
\hline & Sometimes & 0.0571 & 0.8020 & -1.5149 & 1.6290 & 0.01 & 0.9433 \\
\hline & Often & 0.5571 & 0.7411 & -0.8954 & 2.0097 & 0.57 & 0.4522 \\
\hline & Always & 0.0000 & 0.0000 & 0.0000 & 0.0000 & & \\
\hline \multirow{5}{*}{$\begin{array}{l}\text { I refer friends and } \\
\text { family to other } \\
\text { sources that are } \\
\text { outside my scope of } \\
\text { practice. }\end{array}$} & Never & 2.1519 & 1.8747 & -1.5224 & 5.8262 & 1.32 & 0.2510 \\
\hline & Rarely & 2.2484 & 2.3276 & -2.3136 & 6.8103 & 0.93 & 0.3341 \\
\hline & Sometimes & 1.0132 & 0.7067 & -0.3719 & 2.3984 & 2.06 & 0.1516 \\
\hline & Often & 0.2707 & 0.5513 & -0.8099 & 1.3513 & 0.24 & 0.6234 \\
\hline & Always & 0.0000 & 0.0000 & 0.0000 & 0.0000 & & \\
\hline \multirow{4}{*}{$\begin{array}{l}\text { I abide by the } \\
\text { Dietetics Code of } \\
\text { Ethics. }\end{array}$} & Rarely & -5.6519 & 2.1249 & -9.8166 & -1.4872 & 7.07 & 0.0078 \\
\hline & Sometimes & -1.4310 & 0.7241 & -2.8503 & -0.0118 & 3.91 & 0.0481 \\
\hline & Often & -1.8644 & 0.6096 & -3.0592 & -0.6696 & 9.35 & 0.0022 \\
\hline & Always & 0.0000 & 0.0000 & 0.0000 & 0.0000 & & \\
\hline \multirow{5}{*}{$\begin{array}{l}\text { I use nutrition } \\
\text { information to } \\
\text { diagnose health } \\
\text { conditions in family } \\
\text { members and } \\
\text { individuals. }\end{array}$} & Never & -2.2509 & 1.0170 & -4.2442 & -0.2576 & 4.90 & 0.0269 \\
\hline & Rarely & -1.1440 & 0.8305 & -2.7717 & 0.4838 & 1.90 & 0.1684 \\
\hline & Sometimes & -1.4033 & 0.8278 & -3.0258 & 0.2192 & 2.87 & 0.0900 \\
\hline & Often & 0.3165 & 0.8948 & -1.4372 & 2.0703 & 0.13 & 0.7235 \\
\hline & Always & 0.0000 & 0.0000 & 0.0000 & 0.0000 & & \\
\hline \multirow{2}{*}{ Major } & Dietetics & -1.1169 & 0.5068 & -2.1102 & -0.1237 & 4.86 & 0.0275 \\
\hline & Human Ntr & 0.0000 & 0.0000 & 0.0000 & 0.0000 & & \\
\hline
\end{tabular}


Table 55 (Part B): END RELATIONSHIP: Eat your favorite junk food.

\begin{tabular}{|l|r|r|}
\hline \multicolumn{3}{|c|}{ END RELATIONSHIP: Eat your favorite junk food. } \\
\hline Source & Chi-Square & Pr > ChiSq \\
\hline $\begin{array}{l}\text { I only deliver information allowed at the student level by the CDR, as } \\
\text { specified in the Dietetic Scope of Practice. }\end{array}$ & 3.19 & 0.3635 \\
\hline $\begin{array}{l}\text { I comprehend the scope of practice. } \\
\text { I refer friends and family to other sources that are outside my scope of } \\
\text { practice. }\end{array}$ & 3.95 & 0.4122 \\
\hline $\begin{array}{l}\text { I abide by the Dietetics Code of Ethics. } \\
\text { I use nutrition information to diagnose health conditions in family } \\
\text { members and individuals. }\end{array}$ & 15.01 & 0.4131 \\
\hline Major & 14.47 & 0.0018 \\
\hline
\end{tabular}


Table 56 (Part A): UNEXPECTED EXPENSE: Call a close family member or friend and talk about it.

\begin{tabular}{|c|c|c|c|c|c|c|c|}
\hline \multicolumn{8}{|c|}{ UNEXPECTED EXPENSE: Call a close family member or friend and talk about it } \\
\hline \multirow{5}{*}{$\begin{array}{l}\text { Parameter } \\
\text { I only deliver } \\
\text { information } \\
\text { allowed at the } \\
\text { student level by } \\
\text { the CDR, as } \\
\text { specified in the } \\
\text { Dietetic Scope of } \\
\text { Practice. }\end{array}$} & \multirow{2}{*}{$\begin{array}{l}\text { Response } \\
\text { Rarely }\end{array}$} & \multirow{2}{*}{$\begin{array}{r}\text { Estimate } \\
24.5865\end{array}$} & \multirow{2}{*}{\begin{tabular}{|l}
$\begin{array}{l}\text { Standard } \\
\text { Error }\end{array}$ \\
1.8438
\end{tabular}} & \multicolumn{2}{|c|}{$\begin{array}{l}\text { Wald 95\% } \\
\text { Confidence Limits }\end{array}$} & \multirow{2}{*}{$\begin{array}{l}\begin{array}{l}\text { Wald } \\
\text { Chi-Square }\end{array} \\
177.81\end{array}$} & \multirow{2}{*}{\begin{tabular}{|l}
$\begin{array}{l}\mathrm{Pr}> \\
\text { ChiSq }\end{array}$ \\
$<.0001$
\end{tabular}} \\
\hline & & & & 20.9727 & 28.2003 & & \\
\hline & Sometimes & 0.5109 & 0.8464 & -1.1480 & 2.1699 & 0.36 & 0.5461 \\
\hline & Often & 0.4987 & 0.8481 & -1.1636 & 2.1609 & 0.35 & 0.5566 \\
\hline & Always & 0.0000 & 0.0000 & 0.0000 & 0.0000 & & \\
\hline \multirow{5}{*}{$\begin{array}{l}\text { I comprehend the } \\
\text { scope of practice. }\end{array}$} & Never & 24.2035 & 159416.0 & -312425 & 312473.8 & 0.00 & 0.9999 \\
\hline & Rarely & 0.7245 & 1.5895 & -2.3908 & 3.8398 & 0.21 & 0.6485 \\
\hline & Sometimes & 0.1633 & 0.8256 & -1.4549 & 1.7815 & 0.04 & 0.8432 \\
\hline & Often & -0.1630 & 0.7055 & -1.5458 & 1.2197 & 0.05 & 0.8172 \\
\hline & Always & 0.0000 & 0.0000 & 0.0000 & 0.0000 & & \\
\hline \multirow{5}{*}{$\begin{array}{l}\text { I refer friends and } \\
\text { family to other } \\
\text { sources that are } \\
\text { outside my scope } \\
\text { of practice. }\end{array}$} & Never & -1.4278 & 1.7739 & -4.9047 & 2.0490 & 0.65 & 0.4209 \\
\hline & Rarely & 23.2951 & 111303.9 & -218128 & 218174.9 & 0.00 & 0.9998 \\
\hline & Sometimes & -1.6571 & 0.7084 & -3.0455 & -0.2687 & 5.47 & 0.0193 \\
\hline & Often & -0.2941 & 0.5609 & -1.3934 & 0.8053 & 0.27 & 0.6001 \\
\hline & Always & 0.0000 & 0.0000 & 0.0000 & 0.0000 & & \\
\hline \multirow{4}{*}{$\begin{array}{l}\text { I abide by the } \\
\text { Dietetics Code of } \\
\text { Ethics. }\end{array}$} & Rarely & -23.8942 & 0.0000 & -23.8942 & -23.8942 & & \\
\hline & Sometimes & 0.5593 & 0.8149 & -1.0379 & 2.1566 & 0.47 & 0.4925 \\
\hline & Often & 0.5763 & 0.6331 & -0.6646 & 1.8171 & 0.83 & 0.3627 \\
\hline & Always & 0.0000 & 0.0000 & 0.0000 & 0.0000 & & \\
\hline \multirow{5}{*}{$\begin{array}{l}\text { I use nutrition } \\
\text { information to } \\
\text { diagnose health } \\
\text { conditions in } \\
\text { family members } \\
\text { and individuals. }\end{array}$} & Never & 0.8697 & 0.9782 & -1.0476 & 2.7870 & 0.79 & 0.3740 \\
\hline & Rarely & 0.3857 & 0.7905 & -1.1638 & 1.9351 & 0.24 & 0.6257 \\
\hline & Sometimes & 0.6256 & 0.7636 & -0.8710 & 2.1222 & 0.67 & 0.4126 \\
\hline & Often & 0.4821 & 0.8476 & -1.1791 & 2.1434 & 0.32 & 0.5695 \\
\hline & Always & 0.0000 & 0.0000 & 0.0000 & 0.0000 & & \\
\hline \multirow{2}{*}{ Major } & Dietetics & 0.2518 & 0.5227 & -0.7726 & 1.2762 & 0.23 & 0.6300 \\
\hline & Human Ntr & 0.0000 & 0.0000 & 0.0000 & 0.0000 & & \\
\hline
\end{tabular}


Table 56 (Part B): UNEXPECTED EXPENSE: Call a close family member or friend and talk about it.

\begin{tabular}{|l|r|r|}
\hline \multicolumn{3}{|c|}{ UNEXPECTED EXPENSE: Call a close family member or friend and talk about it } \\
\hline Source & Chi-Square & $\mathrm{Pr}>$ ChiSq \\
\hline $\begin{array}{l}\text { I only deliver information allowed at the student level by the CDR, as } \\
\text { specified in the Dietetic Scope of Practice. }\end{array}$ & 2.18 & 0.5366 \\
\hline I comprehend the scope of practice. & 2.11 & 0.7152 \\
\hline $\begin{array}{l}\text { I refer friends and family to other sources that are outside my scope of } \\
\text { practice. }\end{array}$ & 8.63 & 0.0710 \\
\hline I abide by the Dietetics Code of Ethics. & 2.66 & 0.4470 \\
\hline $\begin{array}{l}\text { I use nutrition information to diagnose health conditions in family } \\
\text { members and individuals. }\end{array}$ & 1.02 & 0.9065 \\
\hline Major & 0.23 & 0.6300 \\
\hline
\end{tabular}


Table 57 (Part A): UNEXPECTED EXPENSE: Cry.

\begin{tabular}{|c|c|c|c|c|c|c|c|}
\hline \multicolumn{8}{|c|}{ UNEXPECTED EXPENSE: Cry. } \\
\hline \multirow{5}{*}{$\begin{array}{l}\text { Parameter } \\
\text { I only deliver } \\
\text { information allowed } \\
\text { at the student level by } \\
\text { the CDR, as specified } \\
\text { in the Dietetic Scope } \\
\text { of Practice. }\end{array}$} & \multirow{2}{*}{$\begin{array}{l}\text { Response } \\
\text { Rarely }\end{array}$} & \multirow{2}{*}{$\begin{array}{r}\text { Estimate } \\
3.2250\end{array}$} & \multirow{2}{*}{$\begin{array}{l}\begin{array}{l}\text { Standard } \\
\text { Error }\end{array} \\
1.8390\end{array}$} & \multicolumn{2}{|c|}{$\begin{array}{l}\text { Wald 95\% } \\
\text { Confidence Limits }\end{array}$} & \multirow{2}{*}{\begin{tabular}{|r}
$\begin{array}{l}\text { Wald } \\
\text { Chi-Square }\end{array}$ \\
3.08
\end{tabular}} & \multirow{2}{*}{$\begin{array}{l}\text { Pr }> \\
\text { ChiSq }\end{array}$} \\
\hline & & & & -0.3793 & 6.8293 & & \\
\hline & Sometimes & -0.2003 & 0.8466 & -1.8597 & 1.4590 & 0.06 & 0.8130 \\
\hline & Often & 0.0248 & 0.8201 & -1.5826 & 1.6323 & 0.00 & 0.9759 \\
\hline & Always & 0.0000 & 0.0000 & 0.0000 & 0.0000 & & \\
\hline \multirow{5}{*}{$\begin{array}{l}\text { I comprehend the } \\
\text { scope of practice. }\end{array}$} & Never & 2.7167 & 1.7557 & -0.7244 & 6.1578 & 2.39 & 0.1218 \\
\hline & Rarely & -1.0052 & 1.2186 & -3.3936 & 1.3832 & 0.68 & 0.4094 \\
\hline & Sometimes & -0.2794 & 0.8228 & -1.8920 & 1.3331 & 0.12 & 0.7341 \\
\hline & Often & 0.3706 & 0.7332 & -1.0665 & 1.8076 & 0.26 & 0.6133 \\
\hline & Always & 0.0000 & 0.0000 & 0.0000 & 0.0000 & & \\
\hline \multirow{5}{*}{$\begin{array}{l}\text { I refer friends and } \\
\text { family to other } \\
\text { sources that are } \\
\text { outside my scope of } \\
\text { practice. }\end{array}$} & Never & -0.3338 & 1.8257 & -3.9121 & 3.2445 & 0.03 & 0.8549 \\
\hline & Rarely & 0.5642 & 1.4875 & -2.3513 & 3.4798 & 0.14 & 0.7045 \\
\hline & Sometimes & 0.0141 & 0.6278 & -1.2164 & 1.2446 & 0.00 & 0.9821 \\
\hline & Often & 0.1510 & 0.5493 & -0.9256 & 1.2276 & 0.08 & 0.7834 \\
\hline & Always & 0.0000 & 0.0000 & 0.0000 & 0.0000 & & \\
\hline \multirow{4}{*}{$\begin{array}{l}\text { I abide by the } \\
\text { Dietetics Code of } \\
\text { Ethics. }\end{array}$} & Rarely & -2.9242 & 2.0636 & -6.9688 & 1.1203 & 2.01 & 0.1565 \\
\hline & Sometimes & 1.1252 & 0.7509 & -0.3466 & 2.5970 & 2.25 & 0.1340 \\
\hline & Often & -0.2029 & 0.5887 & -1.3567 & 0.9509 & 0.12 & 0.7304 \\
\hline & Always & 0.0000 & 0.0000 & 0.0000 & 0.0000 & & \\
\hline \multirow{5}{*}{$\begin{array}{l}\text { I use nutrition } \\
\text { information to } \\
\text { diagnose health } \\
\text { conditions in family } \\
\text { members and } \\
\text { individuals. }\end{array}$} & Never & 1.5628 & 1.1141 & -0.6207 & 3.7464 & 1.97 & 0.1607 \\
\hline & Rarely & 0.4407 & 0.8506 & -1.2265 & 2.1079 & 0.27 & 0.6044 \\
\hline & Sometimes & 0.3460 & 0.8208 & -1.2627 & 1.9547 & 0.18 & 0.6733 \\
\hline & Often & 1.6165 & 0.9184 & -0.1835 & 3.4165 & 3.10 & 0.0784 \\
\hline & Always & 0.0000 & 0.0000 & 0.0000 & 0.0000 & & \\
\hline \multirow{2}{*}{ Major } & Dietetics & 0.4279 & 0.5182 & -0.5877 & 1.4435 & 0.68 & 0.4089 \\
\hline & Human Ntr & 0.0000 & 0.0000 & 0.0000 & 0.0000 & & \\
\hline
\end{tabular}


Table 57 (Part B): UNEXPECTED EXPENSE: Cry.

\begin{tabular}{|l|r|r|}
\hline \multicolumn{3}{|c|}{ UNEXPECTED EXPENSE: Cry. } \\
\hline Source & Chi-Square & Pr > ChiSq \\
\hline $\begin{array}{l}\text { I only deliver information allowed at the student level by the CDR, as } \\
\text { specified in the Dietetic Scope of Practice. }\end{array}$ & 4.10 & 0.2509 \\
\hline $\begin{array}{l}\text { I comprehend the scope of practice. } \\
\text { I refer friends and family to other sources that are outside my scope of } \\
\text { practice. }\end{array}$ & 0.05 & 0.2826 \\
\hline $\begin{array}{l}\text { I abide by the Dietetics Code of Ethics. } \\
\text { I use nutrition information to diagnose health conditions in family } \\
\text { members and individuals. }\end{array}$ & 7.81 & 0.9924 \\
\hline Major & 7.50 & 0.1118 \\
\hline
\end{tabular}


Table 57 (Part A): UNEXPECTED EXPENSE: Go to the gym or exercise outdoors.

\begin{tabular}{|c|c|c|c|c|c|c|c|}
\hline \multicolumn{8}{|c|}{ UNEXPECTED EXPENSE: Go to the gym or exercise outdoors. } \\
\hline \multirow{5}{*}{$\begin{array}{l}\text { Parameter } \\
\text { I only deliver } \\
\text { information allowed } \\
\text { at the student level by } \\
\text { the CDR, as specified } \\
\text { in the Dietetic Scope } \\
\text { of Practice. }\end{array}$} & \multirow{2}{*}{$\begin{array}{l}\text { Response } \\
\text { Rarely }\end{array}$} & \multirow{2}{*}{$\begin{array}{r}\text { Estimate } \\
-0.8274\end{array}$} & \multirow{2}{*}{\begin{tabular}{|l|}
$\begin{array}{l}\text { Standard } \\
\text { Error }\end{array}$ \\
2.4023
\end{tabular}} & \multicolumn{2}{|c|}{$\begin{array}{l}\text { Wald 95\% } \\
\text { Confidence Limits }\end{array}$} & \multirow{2}{*}{\begin{tabular}{|r} 
Wald \\
Chi-Square \\
0.12
\end{tabular}} & \multirow{2}{*}{$\begin{array}{l}\begin{array}{l}\text { Pr }> \\
\text { ChiSq }\end{array} \\
0.7305\end{array}$} \\
\hline & & & & -5.5359 & 3.8810 & & \\
\hline & Sometimes & 0.3646 & 0.9490 & -1.4955 & 2.2246 & 0.15 & 0.7009 \\
\hline & Often & 0.2603 & 0.9141 & -1.5312 & 2.0518 & 0.08 & 0.7758 \\
\hline & Always & 0.0000 & 0.0000 & 0.0000 & 0.0000 & & \\
\hline \multirow{5}{*}{$\begin{array}{l}\text { I comprehend the } \\
\text { scope of practice. }\end{array}$} & Never & 0.4883 & 1.7762 & -2.9930 & 3.9696 & 0.08 & 0.7834 \\
\hline & Rarely & -0.9977 & 1.3068 & -3.5591 & 1.5636 & 0.58 & 0.4452 \\
\hline & Sometimes & -1.1848 & 0.8660 & -2.8822 & 0.5126 & 1.87 & 0.1713 \\
\hline & Often & -0.5036 & 0.7903 & -2.0524 & 1.0453 & 0.41 & 0.5240 \\
\hline & Always & 0.0000 & 0.0000 & 0.0000 & 0.0000 & & \\
\hline \multirow{5}{*}{$\begin{array}{l}\text { I refer friends and } \\
\text { family to other } \\
\text { sources that are } \\
\text { outside my scope of } \\
\text { practice. }\end{array}$} & Never & -1.1894 & 1.8993 & -4.9121 & 33 & 0.39 & 0.5312 \\
\hline & Rarely & -3.2029 & 1.5859 & -6.3112 & -0.0946 & 4.08 & 0.043 \\
\hline & Sometimes & -0.1970 & 0.7143 & -1.5970 & 1.2029 & 0.08 & 0.7827 \\
\hline & Often & -0.6148 & 0.6142 & -1.8186 & 0.5890 & 1.00 & 0.3168 \\
\hline & Always & 0.0000 & 0.0000 & 0.0000 & 0.0000 & & \\
\hline \multirow{4}{*}{$\begin{array}{l}\text { I abide by the } \\
\text { Dietetics Code of } \\
\text { Ethics. }\end{array}$} & Rarely & 0.7962 & 2.4712 & -4.0472 & 5.6397 & 0.10 & 0.7473 \\
\hline & Sometimes & -0.5107 & 0.7825 & -2.0444 & 1.0231 & 0.43 & 0.5140 \\
\hline & Often & 0.0365 & 0.6386 & -1.2152 & 1.2882 & 0.00 & 0.954 \\
\hline & Always & 0.0000 & 0.0000 & 0.0000 & 0.0000 & & \\
\hline \multirow{5}{*}{$\begin{array}{l}\text { I use nutrition } \\
\text { information to } \\
\text { diagnose health } \\
\text { conditions in family } \\
\text { members and } \\
\text { individuals. }\end{array}$} & Never & -0.5617 & 1.0710 & -2.6608 & 1.5374 & 0.28 & 0.599 \\
\hline & Rarely & -0.3688 & 0.7996 & -1.9360 & 1.1984 & 0.21 & 0.6446 \\
\hline & Sometimes & -0.2832 & 0.8045 & -1.8599 & 1.2936 & 0.12 & 0.7249 \\
\hline & Often & -0.2463 & 0.8529 & -1.9179 & 1.4254 & 0.08 & 0.7728 \\
\hline & Always & 0.0000 & 0.0000 & 0.0000 & 0.0000 & & \\
\hline \multirow{2}{*}{ Major } & Dietetics & 0.4901 & 0.4907 & -0.4716 & 1.4518 & 1.00 & 0.3178 \\
\hline & Human Ntr & 0.0000 & 0.0000 & 0.0000 & 0.0000 & & \\
\hline
\end{tabular}


Table 57 (Part B): UNEXPECTED EXPENSE: Go to the gym or exercise outdoors.

\begin{tabular}{|l|r|r|}
\hline \multicolumn{3}{|c|}{ UNEXPECTED EXPENSE: Go to the gym or exercise outdoors. } \\
\hline Source & Chi-Square & Pr > ChiSq \\
\hline $\begin{array}{l}\text { I only deliver information allowed at the student level by the CDR, as } \\
\text { specified in the Dietetic Scope of Practice. }\end{array}$ & 0.40 & 0.9395 \\
\hline $\begin{array}{l}\text { I comprehend the scope of practice. } \\
\text { I refer friends and family to other sources that are outside my scope of } \\
\text { practice. }\end{array}$ & 2.86 & 0.5809 \\
\hline $\begin{array}{l}\text { I abide by the Dietetics Code of Ethics. } \\
\text { I use nutrition information to diagnose health conditions in family } \\
\text { members and individuals. }\end{array}$ & 1.17 & 0.3123 \\
\hline Major & 0.35 & 0.9865 \\
\hline
\end{tabular}


Table 58: UNEXPECTED EXPENSE: Drink alcoholic beverages.

\begin{tabular}{|c|c|c|c|c|c|c|c|}
\hline \multicolumn{8}{|c|}{ UNEXPECTED EXPENSES: Drink alcoholic beverages. } \\
\hline \multirow{5}{*}{$\begin{array}{l}\text { Parameter } \\
\text { I only deliver } \\
\text { information } \\
\text { allowed at the } \\
\text { student level by the } \\
\text { CDR, as specified in } \\
\text { the Dietetic Scope } \\
\text { of Practice. }\end{array}$} & \multirow{2}{*}{$\begin{array}{l}\text { Response } \\
\text { Rarely }\end{array}$} & \multirow{2}{*}{\begin{tabular}{|l} 
Estimate \\
25.9130
\end{tabular}} & \multirow{2}{*}{\begin{tabular}{|l|l}
$\begin{array}{l}\text { Standard } \\
\text { Error }\end{array}$ \\
1.8436
\end{tabular}} & \multicolumn{2}{|c|}{$\begin{array}{l}\text { Wald 95\% } \\
\text { Confidence Limits }\end{array}$} & \multirow{2}{*}{\begin{tabular}{|r}
$\begin{array}{l}\text { Wald } \\
\text { Chi-Square }\end{array}$ \\
197.57
\end{tabular}} & \multirow{2}{*}{$\begin{array}{l}\begin{array}{l}\text { Pr }> \\
\text { ChiSq }\end{array} \\
<.0001\end{array}$} \\
\hline & & & & 22.2996 & 29.5263 & & \\
\hline & Sometimes & 0.0149 & 0.8688 & -1.6880 & 1.7178 & 0.00 & 0.9863 \\
\hline & Often & -0.0120 & 0.8892 & -1.7548 & 1.7308 & 0.00 & 0.9892 \\
\hline & Always & 0.0000 & 0.0000 & 0.0000 & 0.0000 & & \\
\hline \multirow{5}{*}{$\begin{array}{l}\text { I comprehend the } \\
\text { scope of practice. }\end{array}$} & Never & 0.9033 & 1.7700 & -2.5658 & 4.3724 & 0.26 & 0.6098 \\
\hline & Rarely & -24.6742 & 0.0000 & -24.6742 & -24.6742 & & \\
\hline & Sometimes & -0.8328 & 0.8846 & -2.5666 & 0.9009 & 0.89 & 0.3465 \\
\hline & Often & 0.4164 & 0.7937 & -1.1392 & 1.9721 & 0.28 & 0.5998 \\
\hline & Always & 0.0000 & 0.0000 & 0.0000 & 0.0000 & & \\
\hline \multirow{5}{*}{$\begin{array}{l}\text { I refer friends and } \\
\text { family to other } \\
\text { sources that are } \\
\text { outside my scope } \\
\text { of practice. }\end{array}$} & Never & -0.1946 & 1.8306 & -3.7825 & 3.3933 & 0.01 & 0.9153 \\
\hline & Rarely & -25.1864 & 166180.9 & -325734 & 325683.4 & 0.00 & 0.9999 \\
\hline & Sometimes & 0.2085 & 0.6960 & -1.1556 & 1.5726 & 0.09 & 0.7645 \\
\hline & Often & 0.2558 & 0.6007 & -0.9215 & 1.4331 & 0.18 & 0.6703 \\
\hline & Always & 0.0000 & 0.0000 & 0.0000 & 0.0000 & & \\
\hline \multirow{4}{*}{$\begin{array}{l}\text { I abide by the } \\
\text { Dietetics Code of } \\
\text { Ethics. }\end{array}$} & Rarely & -48.6193 & 172737.6 & -338608 & 338510.9 & 0.00 & 0.9998 \\
\hline & Sometimes & 0.8271 & 0.7926 & -0.7264 & 2.3805 & 1.09 & 0.2967 \\
\hline & Often & 0.1674 & 0.6494 & -1.1054 & 1.4403 & 0.07 & 0.7965 \\
\hline & Always & 0.0000 & 0.0000 & 0.0000 & 0.0000 & & \\
\hline \multirow{5}{*}{$\begin{array}{l}\text { I use nutrition } \\
\text { information to } \\
\text { diagnose health } \\
\text { conditions in } \\
\text { family members } \\
\text { and individuals. }\end{array}$} & Never & 0.4246 & 1.0193 & -1.5732 & 2.4223 & 0.17 & 0.6770 \\
\hline & Rarely & -0.3650 & 0.8601 & -2.0507 & 1.3207 & 0.18 & 0.6713 \\
\hline & Sometimes & -0.3721 & 0.8118 & -1.9633 & 1.2190 & 0.21 & 0.6467 \\
\hline & Often & -0.1102 & 0.8851 & -1.8450 & 1.6246 & 0.02 & 0.9009 \\
\hline & Always & 0.0000 & 0.0000 & 0.0000 & 0.0000 & & \\
\hline \multirow{2}{*}{ Major } & Dietetics & 0.4942 & 0.5274 & -0.5396 & 1.5279 & 0.88 & 0.3488 \\
\hline & Human Ntr & 0.0000 & 0.0000 & 0.0000 & 0.0000 & & \\
\hline
\end{tabular}


Table 59 (Part A): UNEXPECTED EXPENSE: Immerse yourself in your schoolwork.

\begin{tabular}{|c|c|c|c|c|c|c|c|}
\hline \multicolumn{8}{|c|}{ UNEXPECTED EXPENSES: Immerse yourself in your schoolwork. } \\
\hline \multirow{5}{*}{$\begin{array}{l}\text { Parameter } \\
\text { I only deliver } \\
\text { information allowed } \\
\text { at the student level } \\
\text { by the CDR, as } \\
\text { specified in the } \\
\text { Dietetic Scope of } \\
\text { Practice. }\end{array}$} & \multirow{2}{*}{$\begin{array}{l}\text { Responses } \\
\text { Rarely }\end{array}$} & \multirow{2}{*}{$\begin{array}{l}\text { Estimate } \\
-27.5225\end{array}$} & \multirow{2}{*}{$\begin{array}{l}\begin{array}{l}\text { Standard } \\
\text { Error }\end{array} \\
1.7352\end{array}$} & \multicolumn{2}{|c|}{$\begin{array}{l}\text { Wald 95\% } \\
\text { Confidence Limits }\end{array}$} & \multirow{2}{*}{\begin{tabular}{|r}
$\begin{array}{l}\text { Wald } \\
\text { Chi-Square }\end{array}$ \\
251.59
\end{tabular}} & \multirow{2}{*}{$\begin{array}{l}\begin{array}{l}\text { Pr }> \\
\text { ChiSq }\end{array} \\
<.0001\end{array}$} \\
\hline & & & & -30.9233 & -24.1216 & & \\
\hline & Sometimes & -1.6796 & 0.8866 & -3.4174 & 0.0581 & 3.59 & 0.0582 \\
\hline & Often & -1.7448 & 0.8970 & -3.5029 & 0.0133 & 3.78 & 0.0518 \\
\hline & Always & 0.0000 & 0.0000 & 0.0000 & 0.0000 & & \\
\hline \multirow{5}{*}{$\begin{array}{l}\text { I comprehend the } \\
\text { scope of practice. }\end{array}$} & Never & 2.6079 & 2.0568 & -1.4233 & 6.6391 & 1.61 & 0.2048 \\
\hline & Rarely & 1.0359 & 1.2769 & -1.4667 & 3.5385 & 0.66 & 0.4172 \\
\hline & Sometimes & -1.0753 & 0.7809 & -2.6057 & 0.4552 & 1.90 & 0.1685 \\
\hline & Often & 0.1192 & 0.6889 & -1.2310 & 1.4694 & 0.03 & 0.8626 \\
\hline & Always & 0.0000 & 0.0000 & 0.0000 & 0.0000 & & \\
\hline \multirow{5}{*}{$\begin{array}{l}\text { I refer friends and } \\
\text { family to other } \\
\text { sources that are } \\
\text { outside my scope of } \\
\text { practice. }\end{array}$} & Never & 0.9639 & 1.8312 & -2.6252 & 4.5530 & 0.28 & 0.5986 \\
\hline & Rarely & -2.8648 & 1.7098 & -6.2160 & 0.4865 & 2.81 & 0.0938 \\
\hline & Sometimes & -0.0476 & 0.6841 & -1.3883 & 1.2932 & 0.00 & 0.9446 \\
\hline & Often & 0.2372 & 0.5765 & -0.8928 & 1.3672 & 0.17 & 0.6808 \\
\hline & Always & 0.0000 & 0.0000 & 0.0000 & 0.0000 & & \\
\hline \multirow{4}{*}{$\begin{array}{l}\text { I abide by the } \\
\text { Dietetics Code of } \\
\text { Ethics. }\end{array}$} & Rarely & 25.5330 & 0.0000 & 25.5330 & 25.5330 & & \\
\hline & Sometimes & 0.8122 & 0.7653 & -0.6877 & 2.3121 & 1.13 & 0.2885 \\
\hline & Often & 1.3452 & 0.6050 & 0.1595 & 2.5310 & 4.94 & 0.0262 \\
\hline & Always & 0.0000 & 0.0000 & 0.0000 & 0.0000 & & \\
\hline \multirow{5}{*}{$\begin{array}{l}\text { I use nutrition } \\
\text { information to } \\
\text { diagnose health } \\
\text { conditions in family } \\
\text { members and } \\
\text { individuals. }\end{array}$} & Never & -0.6600 & 1.0362 & -2.6909 & 1.3709 & 0.41 & 0.5241 \\
\hline & Rarely & -0.0236 & 0.8239 & -1.6384 & 1.5913 & 0.00 & 0.9772 \\
\hline & Sometimes & 1.3079 & 0.8388 & -0.3360 & 2.9519 & 2.43 & 0.1189 \\
\hline & Often & 0.4640 & 0.9075 & -1.3147 & 2.2426 & 0.26 & 0.6092 \\
\hline & Always & 0.0000 & 0.0000 & 0.0000 & 0.0000 & & \\
\hline \multirow{2}{*}{ Major } & Dietetics & 0.0967 & 0.5049 & -0.8929 & 1.0863 & 0.04 & 0.8481 \\
\hline & Human Ntr & 0.0000 & 0.0000 & 0.0000 & 0.0000 & & \\
\hline
\end{tabular}


Table 59 (Part B): UNEXPECTED EXPENSE: Immerse yourself in your schoolwork.

\begin{tabular}{|l|r|r|}
\hline \multicolumn{3}{|c|}{ UNEXPECTED EXPENSES: Immerse yourself in your schoolwork. } \\
\hline Source & Chi-Square & Pr > ChiSq \\
\hline $\begin{array}{l}\text { I only deliver information allowed at the student level by the CDR, as } \\
\text { specified in the Dietetic Scope of Practice. }\end{array}$ & 11.14 & 0.0110 \\
\hline I comprehend the scope of practice. & 8.64 & 0.0709 \\
\hline $\begin{array}{l}\text { I refer friends and family to other sources that are outside my scope of } \\
\text { practice. }\end{array}$ & 4.34 & 0.3618 \\
\hline I abide by the Dietetics Code of Ethics. & 8.69 & 0.0337 \\
\hline $\begin{array}{l}\text { I use nutrition information to diagnose health conditions in family } \\
\text { members and individuals. }\end{array}$ & 9.13 & 0.0579 \\
\hline Major & 0.04 & 0.8481 \\
\hline
\end{tabular}


Table 60 (Part A): UNEXPECTED EXPENSE: Eat your favorite junk food.

\begin{tabular}{|c|c|c|c|c|c|c|c|}
\hline \multicolumn{8}{|c|}{ UNEXPECTED EXPENSE: Eat your favorite junk food. } \\
\hline \multirow{5}{*}{$\begin{array}{l}\text { Parameter } \\
\text { I only deliver } \\
\text { information } \\
\text { allowed at the } \\
\text { student level by the } \\
\text { CDR, as specified in } \\
\text { the Dietetic Scope } \\
\text { of Practice. }\end{array}$} & \multirow{2}{*}{$\begin{array}{l}\text { Response } \\
\text { Rarely }\end{array}$} & \multirow{2}{*}{$\begin{array}{r}\text { Estimate } \\
1.1047\end{array}$} & \multirow{2}{*}{\begin{tabular}{|l}
$\begin{array}{l}\text { Standard } \\
\text { Error }\end{array}$ \\
2.2042
\end{tabular}} & \multicolumn{2}{|c|}{$\begin{array}{l}\text { Wald 95\% } \\
\text { Confidence Limits }\end{array}$} & \multirow{2}{*}{\begin{tabular}{|r}
$\begin{array}{l}\text { Wald } \\
\text { Chi-Square }\end{array}$ \\
0.25
\end{tabular}} & \multirow{2}{*}{\begin{tabular}{|l|}
$\begin{array}{l}\mathrm{Pr}> \\
\text { ChiSq }\end{array}$ \\
0.6163
\end{tabular}} \\
\hline & & & & -3.2154 & 5.4247 & & \\
\hline & Sometimes & 0.1377 & 0.8630 & -1.5538 & 1.8292 & 0.03 & 0.8732 \\
\hline & Often & 0.1168 & 0.8967 & -1.6408 & 1.8744 & 0.02 & 0.8964 \\
\hline & Always & 0.0000 & 0.0000 & 0.0000 & 0.0000 & & \\
\hline \multirow{5}{*}{$\begin{array}{l}\text { I comprehend the } \\
\text { scope of practice. }\end{array}$} & Never & 3.6595 & 2.0392 & -0.3373 & 7.6562 & 3.22 & 0.0727 \\
\hline & Rarely & -0.0003 & 1.3186 & -2.5847 & 2.5842 & 0.00 & 0.9998 \\
\hline & Sometimes & -1.1210 & 0.8458 & -2.7788 & 0.5368 & 1.76 & 0.1851 \\
\hline & Often & 0.5621 & 0.7414 & -0.8910 & 2.0153 & 0.57 & 0.4483 \\
\hline & Always & 0.0000 & 0.0000 & 0.0000 & 0.0000 & & \\
\hline \multirow{5}{*}{$\begin{array}{l}\text { I refer friends and } \\
\text { family to other } \\
\text { sources that are } \\
\text { outside my scope of } \\
\text { practice. }\end{array}$} & Never & 2.5269 & 1.8418 & -1.0830 & 6.1367 & 1.88 & 0.1701 \\
\hline & Rarely & -0.3776 & 1.6109 & -3.5349 & 2.7797 & 0.05 & 0.8147 \\
\hline & Sometimes & 0.8148 & 0.7123 & -0.5814 & 2.2109 & 1.31 & 0.2527 \\
\hline & Often & 0.7674 & 0.5707 & -0.3511 & 1.8860 & 1.81 & 0.1787 \\
\hline & Always & 0.0000 & 0.0000 & 0.0000 & 0.0000 & & \\
\hline \multirow{4}{*}{$\begin{array}{l}\text { I abide by the } \\
\text { Dietetics Code of } \\
\text { Ethics. }\end{array}$} & Rarely & -25.6805 & 100448.3 & -196901 & 196849.3 & 0.00 & 0.9998 \\
\hline & Sometimes & 0.2578 & 0.7729 & -1.2571 & 1.7728 & 0.11 & 0.7387 \\
\hline & Often & -0.8119 & 0.6399 & -2.0661 & 0.4423 & 1.61 & 0.2045 \\
\hline & Always & 0.0000 & 0.0000 & 0.0000 & 0.0000 & & \\
\hline \multirow{5}{*}{$\begin{array}{l}\text { I use nutrition } \\
\text { information to } \\
\text { diagnose health } \\
\text { conditions in family } \\
\text { members and } \\
\text { individuals. }\end{array}$} & Never & 0.1315 & 0.9719 & -1.7735 & 2.0365 & 0.02 & 0.8924 \\
\hline & Rarely & -0.1139 & 0.7979 & -1.6778 & 1.4500 & 0.02 & 0.8865 \\
\hline & Sometimes & 0.2782 & 0.7884 & -1.2671 & 1.8235 & 0.12 & 0.7242 \\
\hline & Often & 1.3020 & 0.8503 & -0.3646 & 2.9685 & 2.34 & 0.1257 \\
\hline & Always & 0.0000 & 0.0000 & 0.0000 & 0.0000 & & \\
\hline \multirow{2}{*}{ Major } & Dietetics & -0.5915 & 0.5195 & -1.6097 & 0.4268 & 1.30 & 0.2549 \\
\hline & Human Ntr & 0.0000 & 0.0000 & 0.0000 & 0.0000 & & \\
\hline
\end{tabular}


Table 60 (Part B): UNEXPECTED EXPENSE: Eat your favorite junk food.

\begin{tabular}{|l|r|r|}
\hline \multicolumn{3}{|c|}{ UNEXPECTED EXPENSE: Eat your favorite junk food. } \\
\hline Source & Chi-Square & Pr > ChiSq \\
\hline $\begin{array}{l}\text { I only deliver information allowed at the student level by the CDR, as } \\
\text { specified in the Dietetic Scope of Practice. }\end{array}$ & 0.25 & 0.9687 \\
\hline I comprehend the scope of practice. & 12.21 & 0.0159 \\
\hline $\begin{array}{l}\text { I refer friends and family to other sources that are outside my scope of } \\
\text { practice. }\end{array}$ & 3.70 & 0.4474 \\
\hline I abide by the Dietetics Code of Ethics. & 7.36 & 0.0612 \\
\hline $\begin{array}{l}\text { I use nutrition information to diagnose health conditions in family } \\
\text { members and individuals. }\end{array}$ & 6.09 & 0.1926 \\
\hline Major & 1.30 & 0.2543 \\
\hline
\end{tabular}


Table 61 (Part A): DEATH OF A FAMILY MEMBER/FRIEND: Call a close family member or friend and talk about it.

\begin{tabular}{|c|c|c|c|c|c|c|c|}
\hline \multirow{5}{*}{$\begin{array}{l}\text { Parameter } \\
\text { I only deliver } \\
\text { information } \\
\text { allowed at the } \\
\text { student level by } \\
\text { the CDR, as } \\
\text { specified in the } \\
\text { Dietetic Scope of } \\
\text { Practice. }\end{array}$} & \multirow{2}{*}{$\begin{array}{l}\text { Response } \\
\text { Rarely }\end{array}$} & \multirow{2}{*}{$\begin{array}{r}\text { Estimate } \\
24.2534\end{array}$} & \multirow{2}{*}{$\begin{array}{l}\text { Standard } \\
\text { Error } \\
1.8144\end{array}$} & \multicolumn{2}{|c|}{$\begin{array}{l}\text { Wald 95\% } \\
\text { Confidence Limits }\end{array}$} & \multirow{2}{*}{$\begin{array}{r}\begin{array}{l}\text { Wald } \\
\text { Chi-Square }\end{array} \\
178.69\end{array}$} & \multirow{2}{*}{$\begin{array}{l}\text { Pr }> \\
\text { ChiSq } \\
<.0001\end{array}$} \\
\hline & & & & 20.6973 & 27.8095 & & \\
\hline & Sometimes & -0.5027 & 0.9138 & -2.2937 & 1.2884 & 0.30 & 0.5823 \\
\hline & Often & -0.7426 & 0.9378 & -2.5805 & 1.0954 & 0.63 & 0.4285 \\
\hline & Always & 0.0000 & 0.0000 & 0.0000 & 0.0000 & & \\
\hline \multirow{5}{*}{$\begin{array}{l}\text { I comprehend the } \\
\text { scope of practice. }\end{array}$} & Never & 23.8587 & 164338.7 & -322074 & 322121.9 & 0.00 & 0.9999 \\
\hline & Rarely & -0.2606 & 1.3887 & -2.9824 & 2.4613 & 0.04 & 0.8512 \\
\hline & Sometimes & 0.5084 & 0.8855 & -1.2271 & 2.2440 & 0.33 & 0.5658 \\
\hline & Often & 0.1003 & 0.7694 & -1.4077 & 1.6083 & 0.02 & 0.8962 \\
\hline & Always & 0.0000 & 0.0000 & 0.0000 & 0.0000 & & \\
\hline \multirow{5}{*}{$\begin{array}{l}\text { I refer friends and } \\
\text { family to other } \\
\text { sources that are } \\
\text { outside my scope } \\
\text { of practice. }\end{array}$} & Never & -1.0531 & 1.8368 & -4.6531 & 2.5469 & 0.33 & 0.5664 \\
\hline & Rarely & 24.6182 & 115654.2 & -226653 & 226702.7 & 0.00 & 0.9998 \\
\hline & Sometimes & -0.7862 & 0.7408 & -2.2381 & 0.6658 & 1.13 & 0.2886 \\
\hline & Often & 0.3509 & 0.6667 & -0.9558 & 1.6577 & 0.28 & 0.5987 \\
\hline & Always & 0.0000 & 0.0000 & 0.0000 & 0.0000 & & \\
\hline \multirow{4}{*}{$\begin{array}{l}\text { I abide by the } \\
\text { Dietetics Code of } \\
\text { Ethics. }\end{array}$} & Rarely & -24.9520 & 0.0000 & -24.9520 & -24.9520 & & \\
\hline & Sometimes & -0.0305 & 0.8733 & -1.7422 & 1.6812 & 0.00 & 0.9722 \\
\hline & Often & -0.2215 & 0.6866 & -1.5673 & 1.1242 & 0.10 & 0.7470 \\
\hline & Always & 0.0000 & 0.0000 & 0.0000 & 0.0000 & & \\
\hline \multirow{5}{*}{$\begin{array}{l}\text { I use nutrition } \\
\text { information to } \\
\text { diagnose health } \\
\text { conditions in } \\
\text { family members } \\
\text { and individuals. }\end{array}$} & Never & 0.4641 & 1.1527 & -1.7953 & 2.7234 & 0.16 & 0.6873 \\
\hline & Rarely & -0.4803 & 0.8382 & -2.1232 & 1.1626 & 0.33 & 0.5667 \\
\hline & Sometimes & -0.6548 & 0.8406 & -2.3024 & 0.9927 & 0.61 & 0.4360 \\
\hline & Often & -0.7062 & 0.9074 & -2.4847 & 1.0723 & 0.61 & 0.4364 \\
\hline & Always & 0.0000 & 0.0000 & 0.0000 & 0.0000 & & \\
\hline \multirow{2}{*}{ Major } & Dietetics & 0.3898 & 0.5092 & -0.6081 & 1.3877 & 0.59 & 0.4439 \\
\hline & Human Ntr & 0.0000 & 0.0000 & 0.0000 & 0.0000 & & \\
\hline
\end{tabular}


Table 61 (Part B): DEATH OF A FAMILY MEMBER/FRIEND: Call a close family member or friend and talk about it.

\begin{tabular}{|l|r|r|}
\hline DEATH OF A FAMILY MEMBER/FRIEND: Call a close family member or friend and talk about it. \\
\hline Source & Chi-Square & Pr > ChiSq \\
\hline $\begin{array}{l}\text { I only deliver information allowed at the student level by the CDR, as } \\
\text { specified in the Dietetic Scope of Practice. }\end{array}$ & 3.40 & 0.3342 \\
\hline I comprehend the scope of practice. & 1.79 & 0.7741 \\
\hline $\begin{array}{l}\text { I refer friends and family to other sources that are outside my scope of } \\
\text { practice. }\end{array}$ & 7.13 & 0.1292 \\
\hline I abide by the Dietetics Code of Ethics. & 2.85 & 0.4151 \\
\hline $\begin{array}{l}\text { I use nutrition information to diagnose health conditions in family } \\
\text { members and individuals. }\end{array}$ & 2.09 & 0.7198 \\
\hline Major & 0.59 & 0.4433 \\
\hline
\end{tabular}


Table 62 (Part A): DEATH OF A FAMILY MEMBER/FRIEND: Cry.

\begin{tabular}{|c|c|c|c|c|c|c|c|}
\hline \multicolumn{8}{|c|}{ DEATH OF A FAMILY MEMBER/FRIEND: Cry. } \\
\hline \multirow{5}{*}{$\begin{array}{l}\text { Parameter } \\
\text { I only deliver } \\
\text { information } \\
\text { allowed at the } \\
\text { student level by } \\
\text { the CDR, as } \\
\text { specified in the } \\
\text { Dietetic Scope of } \\
\text { Practice. }\end{array}$} & \multirow{2}{*}{$\begin{array}{l}\text { Response } \\
\text { Rarely }\end{array}$} & \multirow{2}{*}{\begin{tabular}{|r|} 
Estimate \\
21.7986
\end{tabular}} & \multirow{2}{*}{\begin{tabular}{|l}
$\begin{array}{l}\text { Standard } \\
\text { Error }\end{array}$ \\
1.8749
\end{tabular}} & \multicolumn{2}{|c|}{$\begin{array}{l}\text { Wald 95\% } \\
\text { Confidence Limits }\end{array}$} & \multirow{2}{*}{$\begin{array}{l}\text { Wald } \\
\text { Chi-Square } \\
135.18\end{array}$} & \multirow{2}{*}{$\begin{array}{l}\text { Pr }> \\
\text { ChiSq } \\
<.0001\end{array}$} \\
\hline & & & & 18.1239 & 25.4733 & & \\
\hline & Sometimes & -1.5757 & 0.9662 & -3.4694 & 0.3179 & 2.66 & 0.1029 \\
\hline & Often & -1.4315 & 0.9887 & -3.3693 & 0.5062 & 2.10 & 0.1476 \\
\hline & Always & 0.0000 & 0.0000 & 0.0000 & 0.0000 & & \\
\hline \multirow{5}{*}{$\begin{array}{l}\text { I comprehend the } \\
\text { scope of practice. }\end{array}$} & Never & 23.9030 & 166885.3 & -327065 & 327113.1 & 0.00 & 0.9999 \\
\hline & Rarely & -0.6083 & 1.6918 & -3.9241 & 2.7075 & 0.13 & 0.7192 \\
\hline & Sometimes & -1.8954 & 1.0320 & -3.9181 & 0.1273 & 3.37 & 0.0663 \\
\hline & Often & -1.1990 & 0.9347 & -3.0311 & 0.6330 & 1.65 & 0.1996 \\
\hline & Always & 0.0000 & 0.0000 & 0.0000 & 0.0000 & & \\
\hline \multirow{5}{*}{$\begin{array}{l}\text { I refer friends and } \\
\text { family to other } \\
\text { sources that are } \\
\text { outside my scope } \\
\text { of practice. }\end{array}$} & Never & -2.1663 & 1.8677 & -5.8270 & 1.4944 & 1.35 & 0.2461 \\
\hline & Rarely & 21.9724 & 117281.5 & -229846 & 229889.5 & 0.00 & 0.9999 \\
\hline & Sometimes & -0.8750 & 0.7482 & -2.3414 & 0.5914 & 1.37 & 0.2422 \\
\hline & Often & -0.4854 & 0.6481 & -1.7556 & 0.7848 & 0.56 & 0.4538 \\
\hline & Always & 0.0000 & 0.0000 & 0.0000 & 0.0000 & & \\
\hline \multirow{4}{*}{$\begin{array}{l}\text { I abide by the } \\
\text { Dietetics Code of } \\
\text { Ethics. }\end{array}$} & Rarely & -21.8187 & 0.0000 & -21.8187 & -21.8187 & & \\
\hline & Sometimes & 1.6625 & 0.8136 & 0.0679 & 3.2571 & 4.18 & 0.0410 \\
\hline & Often & 0.6923 & 0.6346 & -0.5515 & 1.9360 & 1.19 & 0.2753 \\
\hline & Always & 0.0000 & 0.0000 & 0.0000 & 0.0000 & & \\
\hline \multirow{5}{*}{$\begin{array}{l}\text { I use nutrition } \\
\text { information to } \\
\text { diagnose health } \\
\text { conditions in } \\
\text { family members } \\
\text { and individuals. }\end{array}$} & Never & 2.7694 & 1.2223 & 0.3736 & 5.1652 & 5.13 & 0.0235 \\
\hline & Rarely & 1.8356 & 1.0007 & -0.1257 & 3.7969 & 3.36 & 0.0666 \\
\hline & Sometimes & 1.5244 & 0.9709 & -0.3785 & 3.4274 & 2.47 & 0.1164 \\
\hline & Often & 2.0973 & 1.0502 & 0.0389 & 4.1557 & 3.99 & 0.0458 \\
\hline & Always & 0.0000 & 0.0000 & 0.0000 & 0.0000 & & \\
\hline \multirow{2}{*}{ Major } & Dietetics & 0.0775 & 0.5399 & -0.9807 & 1.1358 & 0.02 & 0.8858 \\
\hline & Human Ntr & 0.0000 & 0.0000 & 0.0000 & 0.0000 & & \\
\hline
\end{tabular}


Table 62 (Part B): DEATH OF A FAMILY MEMBER/FRIEND: Cry.

\begin{tabular}{|l|r|r|}
\hline \multicolumn{3}{|c|}{ DEATH OF A FAMILY MEMBER/FRIEND: Cry. } \\
\hline Source & Chi-Square & Pr > ChiSq \\
\hline $\begin{array}{l}\text { I only deliver information allowed at the student level by the CDR, as } \\
\text { specified in the Dietetic Scope of Practice. }\end{array}$ & 3.81 & 0.2832 \\
\hline I comprehend the scope of practice. & 6.46 & 0.1676 \\
\hline $\begin{array}{l}\text { I refer friends and family to other sources that are outside my scope of } \\
\text { practice. }\end{array}$ & 2.98 & 0.5615 \\
\hline $\begin{array}{l}\text { I abide by the Dietetics Code of Ethics. } \\
\text { I use nutrition information to diagnose health conditions in family } \\
\text { members and individuals. }\end{array}$ & 5.17 & 0.1598 \\
\hline Major & 6.49 & 0.1656 \\
\hline
\end{tabular}


Table 63 (Part A): DEATH OF A FAMILY MEMBER/FRIEND: Go to the gym or exercise outdoors.

\begin{tabular}{|c|c|c|c|c|c|c|c|}
\hline \multicolumn{8}{|c|}{ DEATH OF A FAMILY MEMBER/FRIEND: Go to the gym or exercise outdoors. } \\
\hline \multirow{5}{*}{$\begin{array}{l}\text { Parameter } \\
\text { I only deliver } \\
\text { information } \\
\text { allowed at the } \\
\text { student level by the } \\
\text { CDR, as specified in } \\
\text { the Dietetic Scope } \\
\text { of Practice. }\end{array}$} & \multirow{2}{*}{\begin{tabular}{|l} 
Response \\
Rarely
\end{tabular}} & \multirow{2}{*}{\begin{tabular}{r|} 
Estimate \\
2.3042
\end{tabular}} & \multirow{2}{*}{$\begin{array}{l}\text { Standard } \\
\text { Error } \\
1.8990\end{array}$} & \multicolumn{2}{|c|}{$\begin{array}{l}\text { Wald 95\% } \\
\text { Confidence Limits }\end{array}$} & \multirow{2}{*}{$\begin{array}{r}\text { Wald } \\
\text { Chi-Square } \\
1.47\end{array}$} & \multirow{2}{*}{ 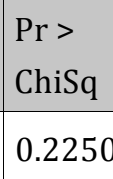 } \\
\hline & & & & -1.4178 & 6.0263 & & \\
\hline & Sometimes & 2.5146 & 0.9594 & 0.6343 & 4.3949 & 6.87 & 0.0088 \\
\hline & Often & 1.9502 & 0.9496 & 0.0889 & 3.8114 & 4.22 & 0.0400 \\
\hline & Always & 0.0000 & 0.0000 & 0.0000 & 0.0000 & & \\
\hline \multirow{5}{*}{$\begin{array}{l}\text { I comprehend the } \\
\text { scope of practice. }\end{array}$} & Never & -1.7081 & 1.7862 & -5.2090 & 1.7929 & 0.91 & 0.3390 \\
\hline & Rarely & 0.1069 & 1.3910 & -2.6194 & 2.8332 & 0.01 & 0.9388 \\
\hline & Sometimes & -0.6324 & 0.7990 & -2.1983 & 0.9336 & 0.63 & 0.4287 \\
\hline & Often & -0.4734 & 0.7245 & -1.8934 & 0.9466 & 0.43 & 0.5135 \\
\hline & Always & 0.0000 & 0.0000 & 0.0000 & 0.0000 & & \\
\hline \multirow{5}{*}{$\begin{array}{l}\text { I refer friends and } \\
\text { family to other } \\
\text { sources that are } \\
\text { outside my scope } \\
\text { of practice. }\end{array}$} & Never & -24.8816 & 66158.05 & -129692 & 129642.5 & 0.00 & 0.9997 \\
\hline & Rarely & -3.8223 & 1.8638 & -7.4753 & -0.1694 & 4.21 & 0.0403 \\
\hline & Sometimes & -1.6135 & 0.6833 & -2.9527 & -0.2743 & 5.58 & 0.0182 \\
\hline & Often & -1.3421 & 0.6101 & -2.5378 & -0.1464 & 4.84 & 0.0278 \\
\hline & Always & 0.0000 & 0.0000 & 0.0000 & 0.0000 & & \\
\hline \multirow{4}{*}{$\begin{array}{l}\text { I abide by the } \\
\text { Dietetics Code of } \\
\text { Ethics. }\end{array}$} & Rarely & -1.6888 & 1.9268 & -5.4653 & 2.0878 & 0.77 & 0.3808 \\
\hline & Sometimes & -0.4460 & 0.7637 & -1.9427 & 1.0508 & 0.34 & 0.5592 \\
\hline & Often & -0.7913 & 0.6301 & -2.0263 & 0.4436 & 1.58 & 0.2091 \\
\hline & Always & 0.0000 & 0.0000 & 0.0000 & 0.0000 & & \\
\hline \multirow{5}{*}{$\begin{array}{l}\text { I use nutrition } \\
\text { information to } \\
\text { diagnose health } \\
\text { conditions in } \\
\text { family members } \\
\text { and individuals. }\end{array}$} & Never & -2.3683 & 1.0313 & -4.3895 & -0.3471 & 5.27 & 0.0216 \\
\hline & Rarely & -1.2155 & 0.7808 & -2.7459 & 0.3148 & 2.42 & 0.1195 \\
\hline & Sometimes & -1.3266 & 0.7917 & -2.8783 & 0.2251 & 2.81 & 0.0938 \\
\hline & Often & -1.2261 & 0.8530 & -2.8979 & 0.4457 & 2.07 & 0.1506 \\
\hline & Always & 0.0000 & 0.0000 & 0.0000 & 0.0000 & & \\
\hline \multirow{2}{*}{ Major } & Dietetics & 0.2586 & 0.4976 & -0.7167 & 1.2338 & 0.27 & 0.6033 \\
\hline & Human Ntr & 0.0000 & 0.0000 & 0.0000 & 0.0000 & & \\
\hline
\end{tabular}


Table 63 (Part B): DEATH OF A FAMILY MEMBER/FRIEND: Go to the gym or exercise outdoors.

\begin{tabular}{|l|r|r|}
\hline \multicolumn{3}{|c|}{ DEATH OF A FAMILY MEMBER/FRIEND: Go to the gym or exercise outdoors. } \\
\hline Source & Chi-Square & Pr > ChiSq \\
\hline $\begin{array}{l}\text { I only deliver information allowed at the student level by the CDR, as } \\
\text { specified in the Dietetic Scope of Practice. }\end{array}$ & 7.28 & 0.0635 \\
\hline $\begin{array}{l}\text { I comprehend the scope of practice. } \\
\text { I refer friends and family to other sources that are outside my scope of } \\
\text { practice. }\end{array}$ & 11.78 & 0.8299 \\
\hline $\begin{array}{l}\text { I abide by the Dietetics Code of Ethics. } \\
\text { I use nutrition information to diagnose health conditions in family } \\
\text { members and individuals. }\end{array}$ & 2.29 & 0.0191 \\
\hline Major & 5.58 & 0.2324 \\
\hline
\end{tabular}


Table 64 (Part A): DEATH OF A FAMILY MEMBER/FRIEND: Drink alcoholic beverages.

\begin{tabular}{|c|c|c|c|c|c|c|c|}
\hline \multicolumn{8}{|c|}{ DEATH OF A FAMILY MEMBER/FRIEND: Drink alcoholic beverages. } \\
\hline \multirow{5}{*}{$\begin{array}{l}\text { Parameter } \\
\text { I only deliver } \\
\text { information } \\
\text { allowed at the } \\
\text { student level by the } \\
\text { CDR, as specified in } \\
\text { the Dietetic Scope } \\
\text { of Practice. }\end{array}$} & \multirow{2}{*}{$\begin{array}{l}\text { Response } \\
\text { Rarely }\end{array}$} & \multirow{2}{*}{\begin{tabular}{r|} 
Estimate \\
25.0988
\end{tabular}} & \multirow{2}{*}{$\begin{array}{l}\begin{array}{l}\text { Standard } \\
\text { Error }\end{array} \\
1.8883\end{array}$} & \multicolumn{2}{|c|}{$\begin{array}{l}\text { Wald 95\% } \\
\text { Confidence Limits }\end{array}$} & \multirow{2}{*}{\begin{tabular}{|r}
$\begin{array}{l}\text { Wald } \\
\text { Chi-Square }\end{array}$ \\
176.68
\end{tabular}} & \multirow{2}{*}{ 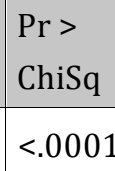 } \\
\hline & & & & 21.3978 & 28.7997 & & \\
\hline & Sometimes & -0.7208 & 0.9015 & -2.4876 & 1.0460 & 0.64 & 0.4240 \\
\hline & Often & -0.5892 & 0.9029 & -2.3588 & 1.1805 & 0.43 & 0.5141 \\
\hline & Always & 0.0000 & 0.0000 & 0.0000 & 0.0000 & & \\
\hline \multirow{5}{*}{$\begin{array}{l}\text { I comprehend the } \\
\text { scope of practice. }\end{array}$} & Never & 1.1508 & 1.7159 & -2.2123 & 4.5138 & 0.45 & 0.5024 \\
\hline & Rarely & -23.3807 & 2.3574 & -28.0010 & -18.7604 & 98.37 & $<.0001$ \\
\hline & Sometimes & 0.3975 & 0.8511 & -1.2707 & 2.0657 & 0.22 & 0.6405 \\
\hline & Often & 0.9032 & 0.7595 & -0.5855 & 2.3919 & 1.41 & 0.2344 \\
\hline & Always & 0.0000 & 0.0000 & 0.0000 & 0.0000 & & \\
\hline \multirow{5}{*}{$\begin{array}{l}\text { I refer friends and } \\
\text { family to other } \\
\text { sources that are } \\
\text { outside my scope of } \\
\text { practice. }\end{array}$} & Never & -23.7302 & 156596.0 & -306946 & 306898.8 & 0.00 & 0.9999 \\
\hline & Rarely & 0.3269 & 1.7525 & -3.1079 & 3.7618 & 0.03 & 0.8520 \\
\hline & Sometimes & 0.0787 & 0.6845 & -1.2629 & 1.4204 & 0.01 & 0.9084 \\
\hline & Often & 0.4361 & 0.5834 & -0.7074 & 1.5795 & 0.56 & 0.4548 \\
\hline & Always & 0.0000 & 0.0000 & 0.0000 & 0.0000 & & \\
\hline \multirow{4}{*}{$\begin{array}{l}\text { I abide by the } \\
\text { Dietetics Code of } \\
\text { Ethics. }\end{array}$} & Rarely & -26.3810 & 0.0000 & -26.3810 & -26.3810 & & \\
\hline & Sometimes & -0.0640 & 0.7618 & -1.5571 & 1.4292 & 0.01 & 0.9331 \\
\hline & Often & -0.1478 & 0.6239 & -1.3706 & 1.0751 & 0.06 & 0.8128 \\
\hline & Always & 0.0000 & 0.0000 & 0.0000 & 0.0000 & & \\
\hline \multirow{5}{*}{$\begin{array}{l}\text { I use nutrition } \\
\text { information to } \\
\text { diagnose health } \\
\text { conditions in family } \\
\text { members and } \\
\text { individuals. }\end{array}$} & Never & 0.1676 & 1.0598 & -1.9095 & 2.2448 & 0.03 & 0.8743 \\
\hline & Rarely & 0.7683 & 0.8339 & -0.8661 & 2.4027 & 0.85 & 0.3569 \\
\hline & Sometimes & 0.2337 & 0.8300 & -1.3931 & 1.8604 & 0.08 & 0.7783 \\
\hline & Often & 0.5227 & 0.8861 & -1.2140 & 2.2593 & 0.35 & 0.5553 \\
\hline & Always & 0.0000 & 0.0000 & 0.0000 & 0.0000 & & \\
\hline \multirow{2}{*}{ Major } & Dietetics & -0.7801 & 0.5276 & -1.8142 & 0.2540 & 2.19 & 0.1393 \\
\hline & Human Ntr & 0.0000 & 0.0000 & 0.0000 & 0.0000 & & \\
\hline
\end{tabular}


Table 64 (Part B): DEATH OF A FAMILY MEMBER/FRIEND: Drink alcoholic beverages.

\begin{tabular}{|l|r|r|}
\hline \multicolumn{3}{|c|}{ DEATH OF A FAMILY MEMBER/FRIEND: Drink alcoholic beverages. } \\
\hline Source & Chi-Square & Pr > ChiSq \\
\hline $\begin{array}{l}\text { I only deliver information allowed at the student level by the CDR, as } \\
\text { specified in the Dietetic Scope of Practice. }\end{array}$ & 6.62 & 0.0850 \\
\hline I comprehend the scope of practice. & 7.11 & 0.1302 \\
\hline $\begin{array}{l}\text { I refer friends and family to other sources that are outside my scope of } \\
\text { practice. }\end{array}$ & 2.02 & 0.7320 \\
\hline I abide by the Dietetics Code of Ethics. & 5.73 & 0.1256 \\
\hline $\begin{array}{l}\text { I use nutrition information to diagnose health conditions in family } \\
\text { members and individuals. }\end{array}$ & 1.46 & 0.8337 \\
\hline Major & 2.20 & 0.1382 \\
\hline
\end{tabular}


Table 65 (Part A): DEATH OF A FAMILY MEMBER/FRIEND: Immerse yourself in your schoolwork.

\begin{tabular}{|c|c|c|c|c|c|c|c|}
\hline \multicolumn{8}{|c|}{ DEATH OF A FAMILY MEMBER/FRIEND: Immerse yourself in your schoolwork. } \\
\hline \multirow{5}{*}{$\begin{array}{l}\text { Parameter } \\
\text { I only deliver } \\
\text { information } \\
\text { allowed at the } \\
\text { student level by } \\
\text { the CDR, as } \\
\text { specified in the } \\
\text { Dietetic Scope of } \\
\text { Practice. }\end{array}$} & \multirow{2}{*}{\begin{tabular}{|l} 
Response \\
Rarely
\end{tabular}} & \multirow{2}{*}{$\begin{array}{r}\text { Estimate } \\
-2.4374\end{array}$} & \multirow{2}{*}{\begin{tabular}{|r}
$\begin{array}{l}\text { Standard } \\
\text { Error }\end{array}$ \\
2.0226
\end{tabular}} & \multicolumn{2}{|c|}{$\begin{array}{l}\text { Wald 95\% } \\
\text { Confidence Limits }\end{array}$} & \multirow{2}{*}{\begin{tabular}{|r} 
Wald \\
Chi-Square \\
1.45
\end{tabular}} & \multirow{2}{*}{ 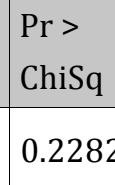 } \\
\hline & & & & -6.4017 & 1.5268 & & \\
\hline & Sometimes & -1.0563 & 0.9002 & -2.8207 & 0.7081 & 1.38 & 0.2407 \\
\hline & Often & -0.7408 & 0.9057 & -2.5159 & 1.0344 & 0.67 & 0.4134 \\
\hline & Always & 0.0000 & 0.0000 & 0.0000 & 0.0000 & & \\
\hline \multirow{5}{*}{$\begin{array}{l}\text { I comprehend the } \\
\text { scope of practice. }\end{array}$} & Never & 1.2373 & 1.7231 & -2.1399 & 4.6145 & 0.52 & 0.4727 \\
\hline & Rarely & 0.7959 & 1.4491 & -2.0444 & 3.6361 & 0.30 & 0.5829 \\
\hline & Sometimes & 0.6629 & 0.8438 & -0.9909 & 2.3167 & 0.62 & 0.4321 \\
\hline & Often & 1.5608 & 0.7927 & 0.0071 & 3.1145 & 3.88 & 0.0490 \\
\hline & Always & 0.0000 & 0.0000 & 0.0000 & 0.0000 & & \\
\hline \multirow{5}{*}{$\begin{array}{l}\text { I refer friends and } \\
\text { family to other } \\
\text { sources that are } \\
\text { outside my scope } \\
\text { of practice. }\end{array}$} & Never & -24.4111 & 77583.97 & -152086 & 152037.4 & 0.00 & 0.9997 \\
\hline & Rarely & -1.3843 & 1.6470 & -4.6123 & 1.8437 & 0.71 & 0.4006 \\
\hline & Sometimes & 0.7809 & 0.7002 & -0.5915 & 2.1533 & 1.24 & 0.2648 \\
\hline & \begin{tabular}{|l|} 
Often \\
\end{tabular} & 0.1387 & 0.6125 & -1.0618 & 1.3392 & 0.05 & 0.8208 \\
\hline & Always & 0.0000 & 0.0000 & 0.0000 & 0.0000 & & \\
\hline \multirow{4}{*}{$\begin{array}{l}\text { I abide by the } \\
\text { Dietetics Code of } \\
\text { Ethics. }\end{array}$} & Rarely & 1.2881 & 1.9546 & -2.5428 & 5.1191 & 0.43 & 0.5099 \\
\hline & Sometimes & -1.5198 & 0.7630 & -3.0152 & -0.0244 & 3.97 & 0.0464 \\
\hline & Often & -0.9594 & 0.6057 & -2.1465 & 0.2276 & 2.51 & 0.1132 \\
\hline & Always & 0.0000 & 0.0000 & 0.0000 & 0.0000 & & \\
\hline \multirow{5}{*}{$\begin{array}{l}\text { I use nutrition } \\
\text { information to } \\
\text { diagnose health } \\
\text { conditions in } \\
\text { family members } \\
\text { and individuals. }\end{array}$} & Never & -0.2874 & 0.9825 & -2.2131 & 1.6382 & 0.09 & 0.7699 \\
\hline & Rarely & -0.6263 & 0.8209 & -2.2353 & 0.9827 & 0.58 & 0.4455 \\
\hline & Sometimes & -0.0483 & 0.8147 & -1.6452 & 1.5485 & 0.00 & 0.9527 \\
\hline & Often & -0.1742 & 0.9019 & -1.9418 & 1.5935 & 0.04 & 0.8469 \\
\hline & Always & 0.0000 & 0.0000 & 0.0000 & 0.0000 & & \\
\hline \multirow{2}{*}{ Major } & Dietetics & -1.0895 & 0.5293 & -2.1268 & -0.0521 & 4.24 & 0.0396 \\
\hline & Human Ntr & 0.0000 & 0.0000 & 0.0000 & 0.0000 & & \\
\hline
\end{tabular}


Table 65 (Part B): DEATH OF A FAMILY MEMBER/FRIEND: Immerse yourself in your schoolwork.

\begin{tabular}{|l|r|r|}
\hline \multicolumn{3}{|c|}{ DEATH OF A FAMILY MEMBER/FRIEND: Immerse yourself in your schoolwork. } \\
\hline Source & Chi-Square & Pr > ChiSq \\
\hline $\begin{array}{l}\text { I only deliver information allowed at the student level by the CDR, as } \\
\text { specified in the Dietetic Scope of Practice. }\end{array}$ & 2.16 & 0.5392 \\
\hline I comprehend the scope of practice. & 5.79 & 0.2155 \\
\hline $\begin{array}{l}\text { I refer friends and family to other sources that are outside my scope of } \\
\text { practice. }\end{array}$ & 6.46 & 0.1671 \\
\hline $\begin{array}{l}\text { I abide by the Dietetics Code of Ethics. } \\
\text { I use nutrition information to diagnose health conditions in family } \\
\text { members and individuals. }\end{array}$ & 5.10 & 0.1644 \\
\hline Major & 4.27 & 0.8664 \\
\hline
\end{tabular}


Table 66 (Part A): DEATH OF A FAMILY MEMBER/FRIEND: Eat your favorite junk food.

\begin{tabular}{|c|c|c|c|c|c|c|c|}
\hline \multicolumn{8}{|c|}{ DEATH OF A FAMILY MEMBER/FRIEND: Eat your favorite junk food. } \\
\hline \multirow{5}{*}{$\begin{array}{l}\text { Parameter } \\
\text { I only deliver } \\
\text { information } \\
\text { allowed at the } \\
\text { student level by the } \\
\text { CDR, as specified in } \\
\text { the Dietetic Scope } \\
\text { of Practice. }\end{array}$} & \multirow{2}{*}{\begin{tabular}{|l} 
Response \\
Rarely
\end{tabular}} & \multirow{2}{*}{$\begin{array}{r}\text { Estimate } \\
3.3859\end{array}$} & \multirow{2}{*}{\begin{tabular}{|l|}
$\begin{array}{l}\text { Standard } \\
\text { Error }\end{array}$ \\
2.2313
\end{tabular}} & \multicolumn{2}{|c|}{$\begin{array}{l}\text { Wald 95\% } \\
\text { Confidence Limits }\end{array}$} & \multirow{2}{*}{$\begin{array}{l}\text { Wald } \\
\text { Chi-Square } \\
2.30\end{array}$} & \multirow{2}{*}{\begin{tabular}{|l|}
$\begin{array}{l}\text { Pr }> \\
\text { ChiSq }\end{array}$ \\
0.1292 \\
\end{tabular}} \\
\hline & & & & -0.9874 & 7.7592 & & \\
\hline & Sometimes & -0.2515 & 0.8889 & -1.9937 & 1.4906 & 0.08 & 0.7772 \\
\hline & Often & -0.4486 & 0.9123 & -2.2366 & 1.3394 & 0.24 & 0.622 \\
\hline & Always & 0.0000 & 0.0000 & 0.0000 & 0.0000 & & \\
\hline \multirow{5}{*}{$\begin{array}{l}\text { I comprehend the } \\
\text { scope of practice. }\end{array}$} & Never & 3.6442 & 2.0084 & -0.2923 & 7.5807 & 3.29 & 0.0696 \\
\hline & Rarely & -2.2949 & 1.5400 & -5.3132 & 0.7234 & 2.22 & 0.1362 \\
\hline & Sometimes & 0.5991 & 0.8290 & -1.0256 & 2.2239 & 0.52 & 0.4699 \\
\hline & Often & 1.1020 & 0.7502 & -0.3684 & 2.5724 & 2.16 & 0.1419 \\
\hline & Always & 0.0000 & 0.0000 & 0.0000 & 0.0000 & & \\
\hline \multirow{5}{*}{$\begin{array}{l}\text { I refer friends and } \\
\text { family to other } \\
\text { sources that are } \\
\text { outside my scope } \\
\text { of practice. }\end{array}$} & Never & -22.1203 & 81101.90 & -158979 & 158934.7 & 0.00 & 0.9998 \\
\hline & Rarely & -1.9024 & 1.7443 & -5.3212 & 1.5163 & 1.19 & 0.2754 \\
\hline & Sometimes & 0.3858 & 0.7079 & -1.0017 & 1.7733 & 0.30 & 0.5858 \\
\hline & Often & 0.1904 & 0.6021 & -0.9897 & 1.3706 & 0.10 & 0.7518 \\
\hline & Always & 0.0000 & 0.0000 & 0.0000 & 0.0000 & & \\
\hline \multirow{4}{*}{$\begin{array}{l}\text { I abide by the } \\
\text { Dietetics Code of } \\
\text { Ethics. }\end{array}$} & Rarely & -5.1038 & 2.3010 & -9.6137 & -0.5939 & 4.92 & 0.0265 \\
\hline & Sometimes & -0.9782 & 0.7905 & -2.5277 & 0.5712 & 1.53 & 0.2159 \\
\hline & Often & -1.4697 & 0.6802 & -2.8029 & -0.1365 & 4.67 & 0.0307 \\
\hline & Always & 0.0000 & 0.0000 & 0.0000 & 0.0000 & & \\
\hline \multirow{5}{*}{$\begin{array}{l}\text { I use nutrition } \\
\text { information to } \\
\text { diagnose health } \\
\text { conditions in } \\
\text { family members } \\
\text { and individuals. }\end{array}$} & Never & -2.9508 & 1.0698 & -5.0475 & -0.8541 & 7.61 & 0.0058 \\
\hline & Rarely & -1.3923 & 0.8312 & -3.0215 & 0.2369 & 2.81 & 0.0939 \\
\hline & Sometimes & -1.9730 & 0.8533 & -3.6453 & -0.3006 & 5.35 & 0.0208 \\
\hline & Often & -0.3447 & 0.9065 & -2.1214 & 1.4320 & 0.14 & 0.7038 \\
\hline & Always & 0.0000 & 0.0000 & 0.0000 & 0.0000 & & \\
\hline \multirow{2}{*}{ Major } & Dietetics & -0.5969 & 0.5070 & -1.5906 & 0.3969 & 1.39 & 0.2391 \\
\hline & Human Ntr & 0.0000 & 0.0000 & 0.0000 & 0.0000 & & \\
\hline
\end{tabular}


Table 66 (Part B): DEATH OF A FAMILY MEMBER/FRIEND: Eat your favorite junk food.

\begin{tabular}{|l|r|r|}
\hline \multicolumn{3}{|c|}{ DEATH OF A FAMILY MEMBER/FRIEND: Eat your favorite junk food. } \\
\hline Source & Chi-Square & Pr > ChiSq \\
\hline $\begin{array}{l}\text { I only deliver information allowed at the student level by the CDR, as } \\
\text { specified in the Dietetic Scope of Practice. }\end{array}$ & 3.28 & 0.3506 \\
\hline $\begin{array}{l}\text { I comprehend the scope of practice. } \\
\text { I refer friends and family to other sources that are outside my scope of } \\
\text { practice. }\end{array}$ & 10.99 & 0.0267 \\
\hline $\begin{array}{l}\text { I abide by the Dietetics Code of Ethics. } \\
\text { I use nutrition information to diagnose health conditions in family } \\
\text { members and individuals. }\end{array}$ & 9.64 & 0.5757 \\
\hline Major & 16.57 & 0.0219 \\
\hline
\end{tabular}


Table 67 (Part A): ACHIEVE/MAINTAIN CERTAIN GPA: Call a close family member or friend and talk about it.

\begin{tabular}{|c|c|c|c|c|c|c|c|}
\hline \multicolumn{8}{|c|}{ ACHIEVE/MAINTAIN CERTAIN GPA: Call a close family member or friend and talk about it. } \\
\hline \multirow{5}{*}{$\begin{array}{l}\text { Parameter } \\
\text { I only deliver } \\
\text { information allowed } \\
\text { at the student level } \\
\text { by the CDR, as } \\
\text { specified in the } \\
\text { Dietetic Scope of } \\
\text { Practice. }\end{array}$} & \multirow{2}{*}{\begin{tabular}{|l|} 
Response \\
Rarely \\
\end{tabular}} & \multirow{2}{*}{\begin{tabular}{|r|} 
Estimate \\
25.2914 \\
\end{tabular}} & \multirow{2}{*}{\begin{tabular}{|r|}
$\begin{array}{l}\text { Standard } \\
\text { Error }\end{array}$ \\
1.5262 \\
\end{tabular}} & \multicolumn{2}{|c|}{$\begin{array}{l}\text { Wald 95\% } \\
\text { Confidence Limits }\end{array}$} & \multirow{2}{*}{\begin{tabular}{|r|}
$\begin{array}{l}\text { Wald } \\
\text { Chi-Square }\end{array}$ \\
274.60 \\
\end{tabular}} & \multirow{2}{*}{$\begin{array}{l}\text { Pr }> \\
\text { ChiSq } \\
<.0001 \\
\end{array}$} \\
\hline & & & & 22.3000 & 28.2827 & & \\
\hline & Sometimes & 0.0968 & 0.8387 & -1.5470 & 1.7405 & 0.01 & 0.9081 \\
\hline & Often & 0.0350 & 0.8295 & -1.5909 & 1.6609 & 0.00 & 0.9664 \\
\hline & Always & 0.0000 & 0.0000 & 0.0000 & 0.0000 & & \\
\hline \multirow{5}{*}{$\begin{array}{l}\text { I comprehend the } \\
\text { scope of practice. }\end{array}$} & Never & 25.6489 & 134465.8 & -263522 & 263573.8 & 0.00 & 0.9998 \\
\hline & Rarely & 1.9764 & 1.2209 & -0.4165 & 4.3693 & 2.62 & 0.1055 \\
\hline & Sometimes & 1.6541 & 0.8175 & 0.0519 & 3.2564 & 4.09 & 0.0430 \\
\hline & Often & 1.0826 & 0.7071 & -0.3032 & 2.4684 & 2.34 & 0.1257 \\
\hline & Always & 0.0000 & 0.0000 & 0.0000 & 0.0000 & & \\
\hline \multirow{5}{*}{$\begin{array}{l}\text { I refer friends and } \\
\text { family to other } \\
\text { sources that are } \\
\text { outside my scope of } \\
\text { practice. }\end{array}$} & Never & -26.2577 & 111346.9 & -218262 & 218209.6 & 0.00 & 0.9998 \\
\hline & Rarely & -0.5099 & 1.7581 & -3.9557 & 2.9358 & 0.08 & 0.7718 \\
\hline & Sometimes & -1.9019 & 0.6950 & -3.2641 & -0.5396 & 7.49 & 0.0062 \\
\hline & Often & -0.9793 & 0.5594 & -2.0757 & 0.1171 & 3.06 & 0.0800 \\
\hline & Always & 0.0000 & 0.0000 & 0.0000 & 0.0000 & & \\
\hline \multirow{4}{*}{$\begin{array}{l}\text { I abide by the } \\
\text { Dietetics Code of } \\
\text { Ethics. }\end{array}$} & Rarely & -25.5638 & 0.0000 & -25.5638 & -25.5638 & & \\
\hline & Sometimes & -1.2331 & 0.7839 & -2.7696 & 0.3033 & 2.47 & 0.1157 \\
\hline & Often & -0.5047 & 0.6106 & -1.7015 & 0.6922 & 0.68 & 0.4086 \\
\hline & Always & 0.0000 & 0.0000 & 0.0000 & 0.0000 & & \\
\hline \multirow{5}{*}{$\begin{array}{l}\text { I use nutrition } \\
\text { information to } \\
\text { diagnose health } \\
\text { conditions in family } \\
\text { members and } \\
\text { individuals. }\end{array}$} & Never & -1.8715 & 0.9863 & -3.8046 & 0.0616 & 3.60 & 0.0578 \\
\hline & Rarely & -1.5870 & 0.8402 & -3.2337 & 0.0597 & 3.57 & 0.0589 \\
\hline & Sometimes & -1.3702 & 0.8146 & -2.9669 & 0.2264 & 2.83 & 0.0926 \\
\hline & Often & -1.1732 & 0.9159 & -2.9683 & 0.6218 & 1.64 & 0.2002 \\
\hline & Always & 0.0000 & 0.0000 & 0.0000 & 0.0000 & & \\
\hline \multirow{2}{*}{ Major } & Dietetics & 0.4183 & 0.5011 & -0.5639 & 1.4005 & 0.70 & 0.4039 \\
\hline & Human Ntr & 0.0000 & 0.0000 & 0.0000 & 0.0000 & & \\
\hline
\end{tabular}


Table 67 (Part B): ACHIEVE/MAINTAIN CERTAIN GPA: Call a close family member or friend and talk about it.

\begin{tabular}{|l|r|r|}
\hline \multicolumn{2}{|c|}{ ACHIEVE/MAINTAIN CERTAIN GPA: Call a close family member or friend and talk about it. } \\
\hline Source & Chi-Square & Pr > ChiSq \\
\hline $\begin{array}{l}\text { I only deliver information allowed at the student level by the CDR, as } \\
\text { specified in the Dietetic Scope of Practice. }\end{array}$ & 4.42 & 0.2196 \\
\hline I comprehend the scope of practice. & 7.04 & 0.1338 \\
\hline $\begin{array}{l}\text { I refer friends and family to other sources that are outside my scope of } \\
\text { practice. }\end{array}$ & 11.63 & 0.0203 \\
\hline I abide by the Dietetics Code of Ethics. & 5.38 & 0.1459 \\
\hline $\begin{array}{l}\text { I use nutrition information to diagnose health conditions in family } \\
\text { members and individuals. }\end{array}$ & 4.91 & 0.2967 \\
\hline Major & 0.70 & 0.4042 \\
\hline
\end{tabular}


Table 68 (Part A): ACHIEVE/MAINTAIN CERTAIN GPA: Cry.

\begin{tabular}{|c|c|c|c|c|c|c|c|}
\hline \multicolumn{8}{|c|}{ ACHIEVE/MAINTAIN CERTAIN GPA: Cry. } \\
\hline \multirow{5}{*}{$\begin{array}{l}\text { Parameter } \\
\text { I only deliver } \\
\text { information } \\
\text { allowed at the } \\
\text { student level by the } \\
\text { CDR, as specified in } \\
\text { the Dietetic Scope } \\
\text { of Practice. }\end{array}$} & \multirow{2}{*}{$\begin{array}{l}\text { Response } \\
\text { Rarely }\end{array}$} & \multirow{2}{*}{$\begin{array}{r}\text { Estimate } \\
1.6245\end{array}$} & \multirow{2}{*}{\begin{tabular}{|l}
$\begin{array}{l}\text { Standard } \\
\text { Error }\end{array}$ \\
1.8108
\end{tabular}} & \multicolumn{2}{|c|}{$\begin{array}{l}\text { Wald 95\% } \\
\text { Confidence Limits }\end{array}$} & \multirow{2}{*}{$\begin{array}{l}\text { Wald } \\
\text { Chi-Square } \\
0.80\end{array}$} & \multirow{2}{*}{\begin{tabular}{|l}
$\begin{array}{l}\mathrm{Pr}> \\
\text { ChiSq }\end{array}$ \\
0.3697
\end{tabular}} \\
\hline & & & & -1.9247 & 5.1737 & & \\
\hline & Sometimes & 0.4491 & 0.8463 & -1.2097 & 2.1078 & 0.28 & 0.5957 \\
\hline & Often & 0.7861 & 0.8431 & -0.8662 & 2.4385 & 0.87 & 0.3511 \\
\hline & Always & 0.0000 & 0.0000 & 0.0000 & 0.0000 & & \\
\hline \multirow{5}{*}{$\begin{array}{l}\text { I comprehend the } \\
\text { scope of practice. }\end{array}$} & Never & -0.2782 & 1.7644 & -3.7364 & 3.1800 & 0.02 & 0.8747 \\
\hline & Rarely & 0.8300 & 1.2742 & -1.6673 & 3.3273 & 0.42 & 0.5148 \\
\hline & Sometimes & -0.0823 & 0.8556 & -1.7591 & 1.5946 & 0.01 & 0.9234 \\
\hline & Often & 0.2002 & 0.7629 & -1.2951 & 1.6954 & 0.07 & 0.7930 \\
\hline & Always & 0.0000 & 0.0000 & 0.0000 & 0.0000 & & \\
\hline \multirow{5}{*}{$\begin{array}{l}\text { I refer friends and } \\
\text { family to other } \\
\text { sources that are } \\
\text { outside my scope } \\
\text { of practice. }\end{array}$} & Never & -23.0439 & 89547.15 & -175532 & 175486.1 & 0.00 & 0.9998 \\
\hline & Rarely & 1.5798 & 1.8459 & -2.0382 & 5.1978 & 0.73 & 0.3921 \\
\hline & Sometimes & 0.5267 & 0.6472 & -0.7419 & 1.7952 & 0.66 & 0.4158 \\
\hline & Often & 0.5255 & 0.5615 & -0.5749 & 1.6260 & 0.88 & 0.3493 \\
\hline & Always & 0.0000 & 0.0000 & 0.0000 & 0.0000 & & \\
\hline \multirow{4}{*}{$\begin{array}{l}\text { I abide by the } \\
\text { Dietetics Code of } \\
\text { Ethics. }\end{array}$} & Rarely & 0.6827 & 1.8808 & -3.0037 & 4.3691 & 0.13 & 0.7166 \\
\hline & Sometimes & -0.1572 & 0.7603 & -1.6473 & 1.3330 & 0.04 & 0.8362 \\
\hline & Often & -0.4268 & 0.6019 & -1.6065 & 0.7528 & 0.50 & 0.4782 \\
\hline & Always & 0.0000 & 0.0000 & 0.0000 & 0.0000 & & \\
\hline \multirow{5}{*}{$\begin{array}{l}\text { I use nutrition } \\
\text { information to } \\
\text { diagnose health } \\
\text { conditions in } \\
\text { family members } \\
\text { and individuals. }\end{array}$} & Never & 0.6996 & 1.0542 & -1.3666 & 2.7657 & 0.44 & 0.5069 \\
\hline & Rarely & 0.8311 & 0.8497 & -0.8343 & 2.4965 & 0.96 & 0.3280 \\
\hline & Sometimes & 1.1813 & 0.8359 & -0.4570 & 2.8195 & 2.00 & 0.1576 \\
\hline & Often & 1.5738 & 0.9084 & -0.2068 & 3.3543 & 3.00 & 0.0832 \\
\hline & Always & 0.0000 & 0.0000 & 0.0000 & 0.0000 & & \\
\hline \multirow{2}{*}{ Major } & Dietetics & 0.1659 & 0.5287 & -0.8703 & 1.2021 & 0.10 & 0.7536 \\
\hline & Human Ntr & 0.0000 & 0.0000 & 0.0000 & 0.0000 & & \\
\hline
\end{tabular}


Table 68 (Part B): ACHIEVE/MAINTAIN CERTAIN GPA: Cry.

\begin{tabular}{|l|r|r|}
\hline \multicolumn{3}{|c|}{ ACHIEVE/MAINTAIN CERTAIN GPA: Cry. } \\
\hline Source & Chi-Square & Pr > ChiSq \\
\hline $\begin{array}{l}\text { I only deliver information allowed at the student level by the CDR, as } \\
\text { specified in the Dietetic Scope of Practice. }\end{array}$ & 1.52 & 0.6771 \\
\hline I comprehend the scope of practice. & 0.97 & 0.9136 \\
\hline $\begin{array}{l}\text { I refer friends and family to other sources that are outside my scope of } \\
\text { practice. }\end{array}$ & 3.33 & 0.5041 \\
\hline I abide by the Dietetics Code of Ethics. & 0.77 & 0.8566 \\
\hline $\begin{array}{l}\text { I use nutrition information to diagnose health conditions in family } \\
\text { members and individuals. }\end{array}$ & 3.80 & 0.4336 \\
\hline Major & 0.10 & 0.7537 \\
\hline
\end{tabular}


Table 69 (Part A): ACHIEVE/MAINTAIN CERTAIN GPA: Go to the gym or exercise outdoors.

\begin{tabular}{|c|c|c|c|c|c|c|c|}
\hline \multicolumn{8}{|c|}{ ACHIEVE/MAINTAIN CERTAIN GPA: Go to the gym or exercise outdoors. } \\
\hline \multirow{5}{*}{$\begin{array}{l}\text { Parameter } \\
\text { I only deliver } \\
\text { information } \\
\text { allowed at the } \\
\text { student level by } \\
\text { the CDR, as } \\
\text { specified in the } \\
\text { Dietetic Scope of } \\
\text { Practice. }\end{array}$} & \multirow{2}{*}{\begin{tabular}{|l} 
Response \\
Rarely
\end{tabular}} & \multirow{2}{*}{$\begin{array}{r}\text { Estimate } \\
5.3271\end{array}$} & \multirow{2}{*}{\begin{tabular}{|r|}
$\begin{array}{l}\text { Standard } \\
\text { Error }\end{array}$ \\
2.1096
\end{tabular}} & \multicolumn{2}{|c|}{$\begin{array}{l}\text { Wald 95\% } \\
\text { Confidence Limits }\end{array}$} & \multirow{2}{*}{\begin{tabular}{|r|}
$\begin{array}{l}\text { Wald } \\
\text { Chi-Square }\end{array}$ \\
6.38
\end{tabular}} & \multirow{2}{*}{$\begin{array}{l}\text { Pr }> \\
\text { ChiSq }\end{array}$} \\
\hline & & & & 1.1922 & 9.4619 & & \\
\hline & Sometimes & 1.9495 & 0.8797 & 0.2252 & 3.6737 & 4.91 & 0.0267 \\
\hline & Often & 1.8479 & 0.9041 & 0.0758 & 3.6199 & 4.18 & 0.0410 \\
\hline & Always & 0.0000 & 0.0000 & 0.0000 & 0.0000 & & \\
\hline \multirow{5}{*}{$\begin{array}{l}\text { I comprehend } \\
\text { the scope of } \\
\text { practice. }\end{array}$} & Never & 24.3484 & 125885.9 & -246708 & 246756.3 & 0.00 & 0.9998 \\
\hline & Rarely & -0.4068 & 1.2596 & -2.8755 & 2.0620 & 0.10 & 0.7467 \\
\hline & Sometimes & 0.1270 & 0.7871 & -1.4156 & 1.6697 & 0.03 & 0.8718 \\
\hline & Often & -0.0963 & 0.7084 & -1.4847 & 1.2921 & 0.02 & 0.8918 \\
\hline & Always & 0.0000 & 0.0000 & 0.0000 & 0.0000 & & \\
\hline \multirow{5}{*}{$\begin{array}{l}\text { I refer friends } \\
\text { and family to } \\
\text { other sources } \\
\text { that are outside } \\
\text { my scope of } \\
\text { practice. }\end{array}$} & Never & -26.1993 & 110737.1 & -217067 & 217014.4 & 0.00 & 0.9998 \\
\hline & Rarely & -1.8561 & 1.6793 & -5.1476 & 1.4353 & 1.22 & 0.2690 \\
\hline & Sometimes & -1.6794 & 0.7268 & -3.1040 & -0.2549 & 5.34 & 0.0209 \\
\hline & Often & -0.8363 & 0.6062 & -2.0245 & 0.3519 & 1.90 & 0.1677 \\
\hline & Always & 0.0000 & 0.0000 & 0.0000 & 0.0000 & & \\
\hline \multirow{4}{*}{$\begin{array}{l}\text { I abide by the } \\
\text { Dietetics Code of } \\
\text { Ethics. }\end{array}$} & Rarely & -4.0416 & 2.1423 & -8.2404 & 0.1572 & 3.56 & 0.0592 \\
\hline & Sometimes & -1.8960 & 0.7913 & -3.4469 & -0.3451 & 5.74 & 0.0166 \\
\hline & Often & -0.8383 & 0.6272 & -2.0675 & 0.3910 & 1.79 & 0.1814 \\
\hline & Always & 0.0000 & 0.0000 & 0.0000 & 0.0000 & & \\
\hline \multirow{5}{*}{$\begin{array}{l}\text { I use nutrition } \\
\text { information to } \\
\text { diagnose health } \\
\text { conditions in } \\
\text { family members } \\
\text { and individuals. }\end{array}$} & Never & -1.4664 & 0.9865 & -3.3999 & 0.4672 & 2.21 & 0.1372 \\
\hline & Rarely & -0.6861 & 0.7913 & -2.2370 & 0.8647 & 0.75 & 0.3859 \\
\hline & Sometimes & -0.5591 & 0.7791 & -2.0862 & 0.9680 & 0.51 & 0.4730 \\
\hline & Often & -0.9350 & 0.8478 & -2.5968 & 0.7267 & 1.22 & 0.2701 \\
\hline & Always & 0.0000 & 0.0000 & 0.0000 & 0.0000 & & \\
\hline \multirow{2}{*}{ Major } & Dietetics & -0.0898 & 0.4798 & -1.0303 & 0.8507 & 0.04 & 0.8516 \\
\hline & Human Ntr & 0.0000 & 0.0000 & 0.0000 & 0.0000 & & \\
\hline
\end{tabular}


Table 69 (Part B): ACHIEVE/MAINTAIN CERTAIN GPA: Go to the gym or exercise outdoors.

\begin{tabular}{|l|r|r|}
\hline \multicolumn{3}{|c|}{ ACHIEVE/MAINTAIN CERTAIN GPA: Go to the gym or exercise outdoors. } \\
\hline Source & Chi-Square & Pr > ChiSq \\
\hline $\begin{array}{l}\text { I only deliver information allowed at the student level by the CDR, as } \\
\text { specified in the Dietetic Scope of Practice. }\end{array}$ & 8.94 & 0.0301 \\
\hline I comprehend the scope of practice. & 2.59 & 0.6291 \\
\hline $\begin{array}{l}\text { I refer friends and family to other sources that are outside my scope of } \\
\text { practice. }\end{array}$ & 9.78 & 0.0444 \\
\hline $\begin{array}{l}\text { I abide by the Dietetics Code of Ethics. } \\
\text { I use nutrition information to diagnose health conditions in family } \\
\text { members and individuals. }\end{array}$ & 7.96 & 0.0468 \\
\hline Major & 2.57 & 0.6323 \\
\hline
\end{tabular}


Table 70: ACHIEVE/MAINTAIN CERTAIN GPA: Drink alcoholic beverages.

\begin{tabular}{|c|c|c|c|c|c|c|c|}
\hline \multicolumn{8}{|c|}{ ACHIEVE/MAINTAIN CERTAIN GPA: Drink alcoholic beverages. } \\
\hline \multirow{5}{*}{$\begin{array}{l}\text { Parameter } \\
\text { I only deliver } \\
\text { information } \\
\text { allowed at the } \\
\text { student level by the } \\
\text { CDR, as specified in } \\
\text { the Dietetic Scope } \\
\text { of Practice. }\end{array}$} & \multirow{2}{*}{\begin{tabular}{|l} 
Response \\
Rarely
\end{tabular}} & \multirow{2}{*}{$\begin{array}{r}\text { Estimate } \\
25.2398\end{array}$} & \multirow{2}{*}{$\begin{array}{l}\begin{array}{l}\text { Standard } \\
\text { Error }\end{array} \\
1.7369\end{array}$} & \multicolumn{2}{|c|}{$\begin{array}{l}\text { Wald 95\% } \\
\text { Confidence Limits }\end{array}$} & \multirow{2}{*}{\begin{tabular}{|r}
$\begin{array}{l}\text { Wald } \\
\text { Chi-Square }\end{array}$ \\
211.15
\end{tabular}} & \multirow{2}{*}{ 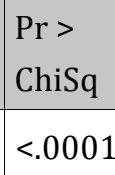 } \\
\hline & & & & 21.8355 & 28.6442 & & \\
\hline & Sometimes & 0.4983 & 0.9403 & -1.3446 & 2.3412 & 0.28 & 0.5961 \\
\hline & Often & 0.2340 & 0.9590 & -1.6456 & 2.1136 & 0.06 & 0.8072 \\
\hline & Always & 0.0000 & 0.0000 & 0.0000 & 0.0000 & & \\
\hline \multirow{5}{*}{$\begin{array}{l}\text { I comprehend the } \\
\text { scope of practice. }\end{array}$} & Never & 1.5030 & 1.7998 & -2.0246 & 5.0305 & 0.70 & 0.4037 \\
\hline & Rarely & -23.7760 & 0.0000 & -23.7760 & -23.7760 & & \\
\hline & Sometimes & 0.0526 & 0.9239 & -1.7582 & 1.8633 & 0.00 & 0.9546 \\
\hline & Often & 1.0602 & 0.8350 & -0.5764 & 2.6968 & 1.61 & 0.2042 \\
\hline & Always & 0.0000 & 0.0000 & 0.0000 & 0.0000 & . & \\
\hline \multirow{5}{*}{$\begin{array}{l}\text { I refer friends and } \\
\text { family to other } \\
\text { sources that are } \\
\text { outside my scope of } \\
\text { practice. }\end{array}$} & Never & -24.9057 & 290427.2 & -569252 & 569201.9 & 0.00 & 0.9999 \\
\hline & Rarely & -24.2914 & 178362.6 & -349609 & 349560.1 & 0.00 & 0.9999 \\
\hline & Sometimes & -0.5396 & 0.7551 & -2.0196 & 0.9403 & 0.51 & 0.4748 \\
\hline & Often & 0.2494 & 0.6223 & -0.9703 & 1.4691 & 0.16 & 0.6885 \\
\hline & Always & 0.0000 & 0.0000 & 0.0000 & 0.0000 & & \\
\hline \multirow{4}{*}{$\begin{array}{l}\text { I abide by the } \\
\text { Dietetics Code of } \\
\text { Ethics. }\end{array}$} & Rarely & -48.3843 & 174798.4 & -342647 & 342550.1 & 0.00 & 0.9998 \\
\hline & Sometimes & 0.2674 & 0.7872 & -1.2756 & 1.8104 & 0.12 & 0.7341 \\
\hline & Often & 0.4529 & 0.6562 & -0.8332 & 1.7391 & 0.48 & 0.4901 \\
\hline & Always & 0.0000 & 0.0000 & 0.0000 & 0.0000 & $\cdot$ & \\
\hline \multirow{5}{*}{$\begin{array}{l}\text { I use nutrition } \\
\text { information to } \\
\text { diagnose health } \\
\text { conditions in family } \\
\text { members and } \\
\text { individuals. }\end{array}$} & Never & 0.0498 & 1.1666 & -2.2366 & 2.3363 & 0.00 & 0.9659 \\
\hline & Rarely & 0.4930 & 0.9138 & -1.2980 & 2.2840 & 0.29 & 0.5895 \\
\hline & Sometimes & 0.6448 & 0.8864 & -1.0925 & 2.3821 & 0.53 & 0.4669 \\
\hline & Often & 0.5962 & 0.9331 & -1.2327 & 2.4250 & 0.41 & 0.5229 \\
\hline & Always & 0.0000 & 0.0000 & 0.0000 & 0.0000 & & \\
\hline \multirow{2}{*}{ Major } & Dietetics & -0.2561 & 0.5432 & -1.3208 & 0.8087 & 0.22 & 0.6374 \\
\hline & Human Ntr & 0.0000 & 0.0000 & 0.0000 & 0.0000 & & \\
\hline
\end{tabular}


Table 71 (Part A): ACHIEVE/MAINTAIN CERTAIN GPA: Immerse yourself in your schoolwork.

\begin{tabular}{|c|c|c|c|c|c|c|c|}
\hline \multicolumn{8}{|c|}{ ACHIEVE/MAINTAIN CERTAIN GPA: Immerse yourself in your schoolwork. } \\
\hline \multirow{5}{*}{$\begin{array}{l}\text { Parameter } \\
\text { I only deliver } \\
\text { information } \\
\text { allowed at the } \\
\text { student level by the } \\
\text { CDR, as specified in } \\
\text { the Dietetic Scope } \\
\text { of Practice. }\end{array}$} & \multirow{2}{*}{$\begin{array}{l}\text { Response } \\
\text { Rarely }\end{array}$} & \multirow{2}{*}{$\begin{array}{r}\text { Estimate } \\
0.6648\end{array}$} & \multirow{2}{*}{$\begin{array}{l}\begin{array}{l}\text { Standard } \\
\text { Error }\end{array} \\
1.9649\end{array}$} & \multicolumn{2}{|c|}{$\begin{array}{l}\text { Wald 95\% } \\
\text { Confidence Limits }\end{array}$} & \multirow{2}{*}{$\begin{array}{l}\text { Wald } \\
\text { Chi-Square } \\
0.11\end{array}$} & \multirow{2}{*}{ 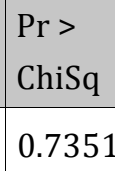 } \\
\hline & & & & -3.1863 & 4.5158 & & \\
\hline & Sometimes & 0.2707 & 0.9149 & -1.5224 & 2.0638 & 0.09 & 0.7673 \\
\hline & Often & 0.8363 & 0.9649 & -1.0550 & 2.7275 & 0.75 & 0.3861 \\
\hline & Always & 0.0000 & 0.0000 & 0.0000 & 0.0000 & & \\
\hline \multirow{5}{*}{$\begin{array}{l}\text { I comprehend the } \\
\text { scope of practice. }\end{array}$} & Never & 21.5471 & 101176.6 & -198281 & 198324.1 & 0.00 & 0.9998 \\
\hline & Rarely & 0.0167 & 1.4124 & -2.7515 & 2.7849 & 0.00 & 0.9906 \\
\hline & Sometimes & -0.6493 & 0.9210 & -2.4544 & 1.1559 & 0.50 & 0.4809 \\
\hline & Often & -0.9327 & 0.8704 & -2.6388 & 0.7733 & 1.15 & 0.2839 \\
\hline & Always & 0.0000 & 0.0000 & 0.0000 & 0.0000 & & \\
\hline \multirow{5}{*}{$\begin{array}{l}\text { I refer friends and } \\
\text { family to other } \\
\text { sources that are } \\
\text { outside my scope of } \\
\text { practice. }\end{array}$} & Never & 23.2088 & 101176.6 & -198279 & 198325.8 & 0.00 & 0.9998 \\
\hline & Rarely & -2.0997 & 2.5054 & -7.0102 & 2.8108 & 0.70 & 0.4020 \\
\hline & Sometimes & -0.7061 & 0.7055 & -2.0888 & 0.6766 & 1.00 & 0.3169 \\
\hline & Often & -0.5808 & 0.6128 & -1.7820 & 0.6203 & 0.90 & 0.3433 \\
\hline & Always & 0.0000 & 0.0000 & 0.0000 & 0.0000 & & \\
\hline \multirow{4}{*}{$\begin{array}{l}\text { I abide by the } \\
\text { Dietetics Code of } \\
\text { Ethics. }\end{array}$} & Rarely & -0.6098 & 2.1222 & -4.7692 & 3.5496 & 0.08 & 0.7739 \\
\hline & Sometimes & -1.2203 & 0.8218 & -2.8311 & 0.3904 & 2.21 & 0.1376 \\
\hline & Often & -0.8692 & 0.6823 & -2.2066 & 0.4681 & 1.62 & 0.2027 \\
\hline & Always & 0.0000 & 0.0000 & 0.0000 & 0.0000 & & \\
\hline \multirow{5}{*}{$\begin{array}{l}\text { I use nutrition } \\
\text { information to } \\
\text { diagnose health } \\
\text { conditions in family } \\
\text { members and } \\
\text { individuals. }\end{array}$} & Never & 0.1121 & 1.0786 & -2.0019 & 2.2261 & 0.01 & 0.9172 \\
\hline & Rarely & 0.2101 & 0.8047 & -1.3670 & 1.7872 & 0.07 & 0.7940 \\
\hline & Sometimes & 1.1182 & 0.8489 & -0.5456 & 2.7820 & 1.74 & 0.1877 \\
\hline & Often & 0.6384 & 0.8905 & -1.1069 & 2.3837 & 0.51 & 0.4734 \\
\hline & Always & 0.0000 & 0.0000 & 0.0000 & 0.0000 & & \\
\hline \multirow{2}{*}{ Major } & Dietetics & -0.7298 & 0.5381 & -1.7844 & 0.3248 & 1.84 & 0.1750 \\
\hline & Human Ntr & 0.0000 & 0.0000 & 0.0000 & 0.0000 & & \\
\hline
\end{tabular}


Table 71 (Part B): ACHIEVE/MAINTAIN CERTAIN GPA: Immerse yourself in your schoolwork.

\begin{tabular}{|l|r|r|}
\hline \multicolumn{3}{|c|}{ ACHIEVE/MAINTAIN CERTAIN GPA: Immerse yourself in your schoolwork. } \\
\hline Source & Chi-Square & Pr > ChiSq \\
\hline $\begin{array}{l}\text { I only deliver information allowed at the student level by the CDR, as } \\
\text { specified in the Dietetic Scope of Practice. }\end{array}$ & 1.27 & 0.7366 \\
\hline I comprehend the scope of practice. & 2.26 & 0.6876 \\
\hline $\begin{array}{l}\text { I refer friends and family to other sources that are outside my scope of } \\
\text { practice. }\end{array}$ & 3.49 & 0.4790 \\
\hline I abide by the Dietetics Code of Ethics. & 2.45 & 0.4846 \\
\hline $\begin{array}{l}\text { I use nutrition information to diagnose health conditions in family } \\
\text { members and individuals. }\end{array}$ & 3.25 & 0.5173 \\
\hline Major & 1.89 & 0.1692 \\
\hline
\end{tabular}


Table 72 (Part A): ACHIEVE/MAINTAIN CERTAIN GPA: Eat your favorite junk food.

\begin{tabular}{|c|c|c|c|c|c|c|c|}
\hline \multicolumn{8}{|c|}{ ACHIEVE/MAINTAIN CERTAIN GPA: Eat your favorite junk food. } \\
\hline \multirow{5}{*}{$\begin{array}{l}\text { Parameter } \\
\text { I only deliver } \\
\text { information } \\
\text { allowed at the } \\
\text { student level by the } \\
\text { CDR, as specified in } \\
\text { the Dietetic Scope } \\
\text { of Practice. }\end{array}$} & \multirow{2}{*}{$\begin{array}{l}\text { Response } \\
\text { Rarely }\end{array}$} & \multirow{2}{*}{$\begin{array}{r}\text { Estimate } \\
1.3696\end{array}$} & \multirow{2}{*}{$\begin{array}{l}\text { Standard } \\
\text { Error } \\
2.4299\end{array}$} & \multicolumn{2}{|c|}{$\begin{array}{l}\text { Wald 95\% } \\
\text { Confidence Limits }\end{array}$} & \multirow{2}{*}{$\begin{array}{l}\text { Wald } \\
\text { Chi-Square } \\
0.32\end{array}$} & \multirow{2}{*}{ 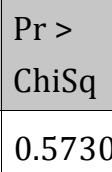 } \\
\hline & & & & -3.3930 & 6.1321 & & \\
\hline & Sometimes & 0.9604 & 0.8954 & -0.7945 & 2.7153 & 1.15 & 0.2834 \\
\hline & Often & 0.2244 & 0.8997 & -1.5391 & 1.9879 & 0.06 & 0.8030 \\
\hline & Always & 0.0000 & 0.0000 & 0.0000 & 0.0000 & & \\
\hline \multirow{5}{*}{$\begin{array}{l}\text { I comprehend the } \\
\text { scope of practice. }\end{array}$} & Never & 26.9952 & 93183.39 & -182609 & 182663.1 & 0.00 & 0.9998 \\
\hline & Rarely & -1.3882 & 1.6841 & -4.6891 & 1.9126 & 0.68 & 0.4098 \\
\hline & Sometimes & 0.2238 & 0.8623 & -1.4663 & 1.9139 & 0.07 & 0.7952 \\
\hline & Often & 1.4587 & 0.7930 & -0.0955 & 3.0129 & 3.38 & 0.0658 \\
\hline & Always & 0.0000 & 0.0000 & 0.0000 & 0.0000 & & \\
\hline \multirow{5}{*}{$\begin{array}{l}\text { I refer friends and } \\
\text { family to other } \\
\text { sources that are } \\
\text { outside my scope of } \\
\text { practice. }\end{array}$} & Never & -23.2841 & 137747.8 & -270004 & 269957.4 & 0.00 & 0.9999 \\
\hline & Rarely & 3.6250 & 2.3956 & -1.0702 & 8.3202 & 2.29 & 0.1302 \\
\hline & Sometimes & 0.6031 & 0.7032 & -0.7752 & 1.9814 & 0.74 & 0.3911 \\
\hline & Often & 0.1516 & 0.5890 & -1.0029 & 1.3061 & 0.07 & 0.7969 \\
\hline & Always & 0.0000 & 0.0000 & 0.0000 & 0.0000 & & \\
\hline \multirow{4}{*}{$\begin{array}{l}\text { I abide by the } \\
\text { Dietetics Code of } \\
\text { Ethics. }\end{array}$} & Rarely & -1.4266 & 2.4088 & -6.1478 & 3.2946 & 0.35 & 0.5537 \\
\hline & Sometimes & -1.1615 & 0.7752 & -2.6809 & 0.3580 & 2.24 & 0.1341 \\
\hline & Often & -1.7149 & 0.6352 & -2.9598 & -0.4700 & 7.29 & 0.0069 \\
\hline & Always & 0.0000 & 0.0000 & 0.0000 & 0.0000 & & \\
\hline \multirow{5}{*}{$\begin{array}{l}\text { I use nutrition } \\
\text { information to } \\
\text { diagnose health } \\
\text { conditions in family } \\
\text { members and } \\
\text { individuals. }\end{array}$} & Never & -0.8861 & 1.0372 & -2.9189 & 1.1468 & 0.73 & 0.3929 \\
\hline & Rarely & 0.7088 & 0.7978 & -0.8548 & 2.2723 & 0.79 & 0.3743 \\
\hline & Sometimes & 0.2635 & 0.8092 & -1.3225 & 1.8494 & 0.11 & 0.7447 \\
\hline & Often & 1.3306 & 0.8555 & -0.3461 & 3.0073 & 2.42 & 0.1198 \\
\hline & Always & 0.0000 & 0.0000 & 0.0000 & 0.0000 & & \\
\hline \multirow{2}{*}{ Major } & Dietetics & -0.4245 & 0.5270 & -1.4574 & 0.6085 & 0.65 & 0.4206 \\
\hline & Human Ntr & 0.0000 & 0.0000 & 0.0000 & 0.0000 & & \\
\hline
\end{tabular}


Table 72 (Part B): ACHIEVE/MAINTAIN CERTAIN GPA: Eat your favorite junk food.

ACHIEVE/MAINTAIN CERTAIN GPA: Eat your favorite junk food.

\begin{tabular}{|l|r|r|}
\hline Source & Chi-Square & Pr > ChiSq \\
\hline $\begin{array}{l}\text { I only deliver information allowed at the student level by the CDR, as } \\
\text { specified in the Dietetic Scope of Practice. }\end{array}$ & 2.34 & 0.5049 \\
\hline I comprehend the scope of practice. & 14.39 & 0.0061 \\
\hline $\begin{array}{l}\text { I refer friends and family to other sources that are outside my scope of } \\
\text { practice. }\end{array}$ & 4.08 & 0.3948 \\
\hline I abide by the Dietetics Code of Ethics. & 7.87 & 0.0488 \\
\hline $\begin{array}{l}\text { I use nutrition information to diagnose health conditions in family } \\
\text { members and individuals. }\end{array}$ & 8.30 & 0.0811 \\
\hline Major & 0.65 & 0.4200 \\
\hline
\end{tabular}


Table 73 (Part A): IRRITATING ROOMMATES/NEIGHBORS: Call a close family member or friend and talk about it.

\begin{tabular}{|c|c|c|c|c|c|c|c|}
\hline \multirow{5}{*}{\begin{tabular}{|l} 
Parameter \\
I only deliver \\
information \\
allowed at the \\
student level by the \\
CDR, as specified in \\
the Dietetic Scope \\
of Practice.
\end{tabular}} & \multirow{2}{*}{$\begin{array}{l}\text { Response } \\
\text { Rarely }\end{array}$} & \multirow{2}{*}{\begin{tabular}{r|} 
Estimate \\
23.3385
\end{tabular}} & \multirow{2}{*}{\begin{tabular}{|r|}
$\begin{array}{l}\text { Standard } \\
\text { Error }\end{array}$ \\
1.6829
\end{tabular}} & \multicolumn{2}{|c|}{$\begin{array}{l}\text { Wald 95\% } \\
\text { Confidence Limits }\end{array}$} & \multirow{2}{*}{\begin{tabular}{|r}
$\begin{array}{l}\text { Wald } \\
\text { Chi-Square }\end{array}$ \\
192.33
\end{tabular}} & \multirow{2}{*}{\begin{tabular}{|l}
$\begin{array}{l}\mathrm{Pr}> \\
\text { ChiSq }\end{array}$ \\
$<.0001$
\end{tabular}} \\
\hline & & & & 20.0401 & 26.6369 & & \\
\hline & Sometimes & 0.6283 & 0.8315 & -1.0014 & 2.2580 & 0.57 & 0.449 \\
\hline & Often & 0.1749 & 0.8396 & -1.4707 & 1.8206 & 0.04 & 0.835 \\
\hline & Always & 0.0000 & 0.0000 & 0.0000 & 0.0000 & & \\
\hline \multirow{5}{*}{$\begin{array}{l}\text { I comprehend the } \\
\text { scope of practice. }\end{array}$} & Never & 24.3556 & 152216.2 & -298314 & 298362.6 & 0.00 & 0.9999 \\
\hline & Rarely & 2.8564 & 1.5797 & -0.2396 & 5.9525 & 3.27 & 0.0706 \\
\hline & Sometimes & 0.9061 & 0.8135 & -0.6883 & 2.5005 & 1.24 & 0.2653 \\
\hline & Often & 0.5025 & 0.7329 & -0.9340 & 1.9389 & 0.47 & 0.4930 \\
\hline & Always & 0.0000 & 0.0000 & 0.0000 & 0.0000 & & \\
\hline \multirow{5}{*}{$\begin{array}{l}\text { I refer friends and } \\
\text { family to other } \\
\text { sources that are } \\
\text { outside my scope of } \\
\text { practice. }\end{array}$} & Never & -1.4826 & 1.8405 & -5.0899 & 2.1248 & 0.65 & 0.4205 \\
\hline & Rarely & -1.2229 & 1.6854 & -4.5262 & 2.0803 & 0.53 & 0.4681 \\
\hline & Sometimes & -2.1162 & 0.7727 & -3.6307 & -0.6017 & 7.50 & 0.0062 \\
\hline & Often & -1.3359 & 0.6080 & -2.5276 & -0.1442 & 4.83 & 0.0280 \\
\hline & Always & 0.0000 & 0.0000 & 0.0000 & 0.0000 & & \\
\hline \multirow{4}{*}{$\begin{array}{l}\text { I abide by the } \\
\text { Dietetics Code of } \\
\text { Ethics. }\end{array}$} & Rarely & -23.5108 & 0.0000 & -23.5108 & -23.5108 & & \\
\hline & Sometimes & -0.3850 & 0.7823 & -1.9183 & 1.1483 & 0.24 & 0.6226 \\
\hline & Often & -0.3957 & 0.6100 & -1.5913 & 0.8000 & 0.42 & 0.5166 \\
\hline & Always & 0.0000 & 0.0000 & 0.0000 & 0.0000 & & \\
\hline \multirow{5}{*}{$\begin{array}{l}\text { I use nutrition } \\
\text { information to } \\
\text { diagnose health } \\
\text { conditions in family } \\
\text { members and } \\
\text { individuals. }\end{array}$} & Never & 0.5937 & 1.0343 & -1.4334 & 2.6209 & 0.33 & 0.5659 \\
\hline & Rarely & -0.4066 & 0.8179 & -2.0096 & 1.1965 & 0.25 & 0.6191 \\
\hline & Sometimes & 0.4093 & 0.7903 & -1.1395 & 1.9582 & 0.27 & 0.6045 \\
\hline & Often & 0.6370 & 0.8958 & -1.1187 & 2.3928 & 0.51 & 0.4770 \\
\hline & Always & 0.0000 & 0.0000 & 0.0000 & 0.0000 & & \\
\hline \multirow{2}{*}{ Major } & Dietetics & 0.7735 & 0.5299 & -0.2651 & 1.8121 & 2.13 & 0.1444 \\
\hline & Human Ntr & 0.0000 & 0.0000 & 0.0000 & 0.0000 & & \\
\hline
\end{tabular}


Table 73 (Part B): IRRITATING ROOMMATES/NEIGHBORS: Call a close family member or friend and talk about it.

\begin{tabular}{|l|r|r|}
\hline \multicolumn{2}{|l|}{ IRRITATING ROOMMATES/NEIGHBORS: Call a close family member or friend and talk about it. } \\
\hline Source & Chi-Square & $\mathrm{Pr}>$ ChiSq \\
\hline $\begin{array}{l}\text { I only deliver information allowed at the student level by the CDR, as } \\
\text { specified in the Dietetic Scope of Practice. }\end{array}$ & 1.73 & 0.6303 \\
\hline $\begin{array}{l}\text { I comprehend the scope of practice. } \\
\text { I refer friends and family to other sources that are outside my scope of } \\
\text { practice. }\end{array}$ & 8.47 & 0.2420 \\
\hline $\begin{array}{l}\text { I abide by the Dietetics Code of Ethics. } \\
\text { I use nutrition information to diagnose health conditions in family } \\
\text { members and individuals. }\end{array}$ & 1.59 & 0.0733 \\
\hline Major & 3.17 & 0.6622 \\
\hline
\end{tabular}


Table 74 (Part A): IRRITATING ROOMMATES/NEIGHBORS: Cry.

\begin{tabular}{|c|c|c|c|c|c|c|c|}
\hline \multicolumn{8}{|c|}{ IRRITATING ROOMMATES/NEIGHBORS: Cry. } \\
\hline \multirow{5}{*}{$\begin{array}{l}\text { Parameter } \\
\text { I only deliver } \\
\text { information } \\
\text { allowed at the } \\
\text { student level by the } \\
\text { CDR, as specified in } \\
\text { the Dietetic Scope } \\
\text { of Practice. }\end{array}$} & \multirow{2}{*}{$\begin{array}{l}\text { Response } \\
\text { Rarely }\end{array}$} & \multirow{2}{*}{$\begin{array}{r}\text { Estimate } \\
2.2259\end{array}$} & \multirow{2}{*}{\begin{tabular}{|l} 
Standard \\
Error
\end{tabular}} & \multicolumn{2}{|c|}{$\begin{array}{l}\text { Wald 95\% } \\
\text { Confidence Limits }\end{array}$} & \multirow{2}{*}{\begin{tabular}{|r}
$\begin{array}{l}\text { Wald } \\
\text { Chi-Square }\end{array}$ \\
0.99
\end{tabular}} & \multirow{2}{*}{$\begin{array}{l}\begin{array}{l}\text { Pr }> \\
\text { ChiSq }\end{array} \\
0.3197\end{array}$} \\
\hline & & & & -2.1581 & 6.6100 & & \\
\hline & Sometimes & 0.1122 & 0.9700 & -1.7889 & 2.0134 & 0.01 & 0.9079 \\
\hline & Often & -0.3697 & 0.9959 & -2.3217 & 1.5823 & 0.14 & 0.7105 \\
\hline & Always & 0.0000 & 0.0000 & 0.0000 & 0.0000 & & \\
\hline \multirow{5}{*}{$\begin{array}{l}\text { I comprehend the } \\
\text { scope of practice. }\end{array}$} & Never & 3.3612 & 1.8637 & -0.2916 & 7.0140 & 3.25 & 0.0713 \\
\hline & Rarely & 1.2387 & 1.3207 & -1.3498 & 3.8272 & 0.88 & 0.3483 \\
\hline & Sometimes & 0.0012 & 1.0083 & -1.9750 & 1.9775 & 0.00 & 0.9990 \\
\hline & Often & 1.2187 & 0.9009 & -0.5471 & 2.9845 & 1.83 & 0.1761 \\
\hline & Always & 0.0000 & 0.0000 & 0.0000 & 0.0000 & & \\
\hline \multirow{5}{*}{$\begin{array}{l}\text { I refer friends and } \\
\text { family to other } \\
\text { sources that are } \\
\text { outside my scope of } \\
\text { practice. }\end{array}$} & Never & -23.8837 & 108005.6 & -211711 & 211663.1 & 0.00 & 0.9998 \\
\hline & Rarely & 0.8354 & 1.7887 & -2.6705 & 4.3412 & 0.22 & 0.6405 \\
\hline & Sometimes & -0.8203 & 0.8542 & -2.4946 & 0.8540 & 0.92 & 0.3369 \\
\hline & Often & 0.5707 & 0.6446 & -0.6927 & 1.8341 & 0.78 & 0.3760 \\
\hline & Always & 0.0000 & 0.0000 & 0.0000 & 0.0000 & & \\
\hline \multirow{4}{*}{$\begin{array}{l}\text { I abide by the } \\
\text { Dietetics Code of } \\
\text { Ethics. }\end{array}$} & Rarely & -1.2156 & 2.3859 & -5.8919 & 3.4607 & 0.26 & 0.6104 \\
\hline & Sometimes & 0.0383 & 0.8925 & -1.7109 & 1.7876 & 0.00 & 0.9658 \\
\hline & Often & 0.4965 & 0.7225 & -0.9195 & 1.9125 & 0.47 & 0.4920 \\
\hline & Always & 0.0000 & 0.0000 & 0.0000 & 0.0000 & & \\
\hline \multirow{5}{*}{$\begin{array}{l}\text { I use nutrition } \\
\text { information to } \\
\text { diagnose health } \\
\text { conditions in family } \\
\text { members and } \\
\text { individuals. }\end{array}$} & Never & 1.0232 & 1.1586 & -1.2476 & 3.2941 & 0.78 & 0.3771 \\
\hline & Rarely & 0.2965 & 0.9118 & -1.4906 & 2.0835 & 0.11 & 0.7451 \\
\hline & Sometimes & -0.1448 & 0.9167 & -1.9415 & 1.6519 & 0.02 & 0.8745 \\
\hline & Often & 0.4599 & 0.9659 & -1.4332 & 2.3530 & 0.23 & 0.6340 \\
\hline & Always & 0.0000 & 0.0000 & 0.0000 & 0.0000 & & \\
\hline \multirow{2}{*}{ Major } & Dietetics & 0.5778 & 0.5982 & -0.5947 & 1.7503 & 0.93 & 0.3341 \\
\hline & Human Ntr & 0.0000 & 0.0000 & 0.0000 & 0.0000 & & \\
\hline
\end{tabular}


Table 74 (Part B): IRRITATING ROOMMATES/NEIGHBORS: Cry.

\begin{tabular}{|l|r|r|}
\hline \multicolumn{3}{|c|}{ IRRITATING ROOMMATES/NEIGHBORS: Cry. } \\
\hline Source & Chi-Square & Pr > ChiSq \\
\hline $\begin{array}{l}\text { I only deliver information allowed at the student level by the CDR, as } \\
\text { specified in the Dietetic Scope of Practice. }\end{array}$ & 1.68 & 0.6410 \\
\hline I comprehend the scope of practice. & 7.82 & 0.0985 \\
\hline $\begin{array}{l}\text { I refer friends and family to other sources that are outside my scope of } \\
\text { practice. }\end{array}$ & 6.31 & 0.1769 \\
\hline I abide by the Dietetics Code of Ethics. & 1.02 & 0.7968 \\
\hline $\begin{array}{l}\text { I use nutrition information to diagnose health conditions in family } \\
\text { members and individuals. }\end{array}$ & 1.73 & 0.7856 \\
\hline Major & 0.95 & 0.3294 \\
\hline
\end{tabular}


Table 75 (Part A): IRRITATING ROOMMATES/NEIGHBORS: Go to the gym or exercise outdoors.

\begin{tabular}{|c|c|c|c|c|c|c|c|}
\hline \multicolumn{8}{|c|}{ IRRITATING ROOMMATES/NEIGHBORS: Go to the gym or exercise outdoors. } \\
\hline \multirow{5}{*}{$\begin{array}{l}\text { Parameter } \\
\text { I only deliver } \\
\text { information } \\
\text { allowed at the } \\
\text { student level by the } \\
\text { CDR, as specified in } \\
\text { the Dietetic Scope } \\
\text { of Practice. }\end{array}$} & \multirow{2}{*}{$\begin{array}{l}\text { Response } \\
\text { Rarely }\end{array}$} & \multirow{2}{*}{$\begin{array}{r}\text { Estimate } \\
0.4055\end{array}$} & \multirow{2}{*}{\begin{tabular}{|l}
$\begin{array}{l}\text { Standard } \\
\text { Error }\end{array}$ \\
2.2141
\end{tabular}} & \multicolumn{2}{|c|}{$\begin{array}{l}\text { Wald 95\% } \\
\text { Confidence Limits }\end{array}$} & \multirow{2}{*}{\begin{tabular}{|r}
$\begin{array}{l}\text { Wald } \\
\text { Chi-Square }\end{array}$ \\
0.03
\end{tabular}} & \multirow{2}{*}{$\begin{array}{l}\begin{array}{l}\text { Pr }> \\
\text { ChiSq }\end{array} \\
0.8547\end{array}$} \\
\hline & & & & -3.9341 & 4.7452 & & \\
\hline & Sometimes & 2.1981 & 0.9481 & 0.3398 & 4.0563 & 5.38 & 0.0204 \\
\hline & Often & 1.6881 & 0.9628 & -0.1990 & 3.5751 & 3.07 & 0.0796 \\
\hline & Always & 0.0000 & 0.0000 & 0.0000 & 0.0000 & & \\
\hline \multirow{5}{*}{$\begin{array}{l}\text { I comprehend the } \\
\text { scope of practice. }\end{array}$} & Never & 24.0183 & 126911.3 & -248718 & 248765.7 & 0.00 & 0.9998 \\
\hline & Rarely & 0.1508 & 1.4540 & -2.6989 & 3.0005 & 0.01 & 0.9174 \\
\hline & Sometimes & -0.7801 & 0.8589 & -2.4635 & 0.9033 & 0.82 & 0.3637 \\
\hline & Often & -0.7662 & 0.7848 & -2.3044 & 0.7720 & 0.95 & 0.3289 \\
\hline & Always & 0.0000 & 0.0000 & 0.0000 & 0.0000 & & \\
\hline \multirow{5}{*}{$\begin{array}{l}\text { I refer friends and } \\
\text { family to other } \\
\text { sources that are } \\
\text { outside my scope of } \\
\text { practice. }\end{array}$} & Never & 0.6091 & 1.8799 & -3.0755 & 4.2936 & 0.10 & 0.7459 \\
\hline & Rarely & -26.8315 & 77147.25 & -151233 & 151179.0 & 0.00 & 0.9997 \\
\hline & Sometimes & -1.0530 & 0.7018 & -2.4284 & 0.3224 & 2.25 & 0.1335 \\
\hline & Often & -1.3647 & 0.6148 & -2.5697 & -0.1596 & 4.93 & 0.0264 \\
\hline & Always & 0.0000 & 0.0000 & 0.0000 & 0.0000 & & \\
\hline \multirow{4}{*}{$\begin{array}{l}\text { I abide by the } \\
\text { Dietetics Code of } \\
\text { Ethics. }\end{array}$} & Rarely & 0.8499 & 2.3040 & -3.6658 & 5.3657 & 0.14 & 0.7122 \\
\hline & Sometimes & -1.6634 & 0.7785 & -3.1892 & -0.1375 & 4.57 & 0.0326 \\
\hline & Often & -0.9759 & 0.6321 & -2.2148 & 0.2629 & 2.38 & 0.1226 \\
\hline & Always & 0.0000 & 0.0000 & 0.0000 & 0.0000 & & \\
\hline \multirow{5}{*}{$\begin{array}{l}\text { I use nutrition } \\
\text { information to } \\
\text { diagnose health } \\
\text { conditions in family } \\
\text { members and } \\
\text { individuals. }\end{array}$} & Never & -1.7677 & 1.0005 & -3.7287 & 0.1933 & 3.12 & 0.0773 \\
\hline & Rarely & -1.7883 & 0.8159 & -3.3873 & -0.1892 & 4.80 & 0.0284 \\
\hline & Sometimes & -1.4442 & 0.8508 & -3.1117 & 0.2233 & 2.88 & 0.0896 \\
\hline & Often & -1.2725 & 0.8761 & -2.9897 & 0.4447 & 2.11 & 0.1464 \\
\hline & Always & 0.0000 & 0.0000 & 0.0000 & 0.0000 & & \\
\hline \multirow{2}{*}{ Major } & Dietetics & 0.1012 & 0.4985 & -0.8758 & 1.0781 & 0.04 & 0.8392 \\
\hline & Human Ntr & 0.0000 & 0.0000 & 0.0000 & 0.0000 & & \\
\hline
\end{tabular}


Table 75 (Part B): IRRITATING ROOMMATES/NEIGHBORS: Go to the gym or exercise outdoors.

\begin{tabular}{|l|r|r|}
\hline \multicolumn{3}{|c|}{ IRRITATING ROOMMATES/NEIGHBORS: Go to the gym or exercise outdoors. } \\
\hline Source & Chi-Square & Pr > ChiSq \\
\hline $\begin{array}{l}\text { I only deliver information allowed at the student level by the CDR, as } \\
\text { specified in the Dietetic Scope of Practice. }\end{array}$ & 5.95 & 0.1141 \\
\hline I comprehend the scope of practice. & 4.01 & 0.4048 \\
\hline $\begin{array}{l}\text { I refer friends and family to other sources that are outside my scope of } \\
\text { practice. }\end{array}$ & 13.08 & 0.0109 \\
\hline I abide by the Dietetics Code of Ethics. & 5.09 & 0.1657 \\
\hline $\begin{array}{l}\text { I use nutrition information to diagnose health conditions in family } \\
\text { members and individuals. }\end{array}$ & 5.47 & 0.2423 \\
\hline Major & 0.04 & 0.8392 \\
\hline
\end{tabular}


Table 76: IRRITATING ROOMMATES/NEIGHBORS: Drink alcoholic beverages.

\begin{tabular}{|c|c|c|c|c|c|c|c|}
\hline \multicolumn{8}{|c|}{ IRRITATING ROOMMATES/NEIGHBORS: Drink alcoholic beverages. } \\
\hline \multirow{5}{*}{$\begin{array}{l}\text { Parameter } \\
\text { I only deliver } \\
\text { information } \\
\text { allowed at the } \\
\text { student level by the } \\
\text { CDR, as specified in } \\
\text { the Dietetic Scope } \\
\text { of Practice. }\end{array}$} & \multirow{2}{*}{$\begin{array}{l}\text { Response } \\
\text { Rarely }\end{array}$} & \multirow{2}{*}{\begin{tabular}{r|} 
Estimate \\
0.6778
\end{tabular}} & \multirow{2}{*}{\begin{tabular}{|l} 
Standard \\
Error
\end{tabular}} & \multicolumn{2}{|c|}{$\begin{array}{l}\text { Wald 95\% } \\
\text { Confidence Limits }\end{array}$} & \multirow{2}{*}{$\begin{array}{l}\text { Wald } \\
\text { Chi-Square } \\
\quad 0.00\end{array}$} & \multirow{2}{*}{\begin{tabular}{|l}
$\begin{array}{l}\mathrm{Pr}> \\
\text { ChiSq }\end{array}$ \\
1.0000
\end{tabular}} \\
\hline & & & & -500641 & 500642.5 & & \\
\hline & Sometimes & -0.1901 & 1.1595 & -2.4627 & 2.0825 & 0.03 & 0.8698 \\
\hline & Often & -0.2789 & 1.1784 & -2.5886 & 2.0309 & 0.06 & 0.8129 \\
\hline & Always & 0.0000 & 0.0000 & 0.0000 & 0.0000 & & \\
\hline \multirow{5}{*}{$\begin{array}{l}\text { I comprehend the } \\
\text { scope of practice. }\end{array}$} & Never & 2.7869 & 1.9173 & -0.9711 & 6.5448 & 2.11 & 0.1461 \\
\hline & Rarely & -25.5601 & 135423.5 & -265451 & 265399.7 & 0.00 & 0.9998 \\
\hline & Sometimes & -1.3044 & 0.9905 & -3.2457 & 0.6368 & 1.73 & 0.1878 \\
\hline & Often & -0.9399 & 0.8672 & -2.6397 & 0.7598 & 1.17 & 0.2784 \\
\hline & Always & 0.0000 & 0.0000 & 0.0000 & 0.0000 & & \\
\hline \multirow{5}{*}{$\begin{array}{l}\text { I refer friends and } \\
\text { family to other } \\
\text { sources that are } \\
\text { outside my scope } \\
\text { of practice. }\end{array}$} & Never & -23.3433 & 318608.5 & -624484 & 624437.8 & 0.00 & 0.9999 \\
\hline & Rarely & -22.0961 & 196009.6 & -384194 & 384149.7 & 0.00 & 0.9999 \\
\hline & Sometimes & 0.6713 & 1.0060 & -1.3004 & 2.6430 & 0.45 & 0.5046 \\
\hline & Often & 1.9340 & 0.8878 & 0.1940 & 3.6740 & 4.75 & 0.0294 \\
\hline & Always & 0.0000 & 0.0000 & 0.0000 & 0.0000 & & \\
\hline \multirow{4}{*}{$\begin{array}{l}\text { I abide by the } \\
\text { Dietetics Code of } \\
\text { Ethics. }\end{array}$} & Rarely & -24.1239 & 316606.9 & -620562 & 620514.0 & 0.00 & 0.9999 \\
\hline & Sometimes & 0.8174 & 1.0464 & -1.2335 & 2.8682 & 0.61 & 0.4347 \\
\hline & Often & 1.4169 & 0.8091 & -0.1689 & 3.0027 & 3.07 & 0.0799 \\
\hline & Always & 0.0000 & 0.0000 & 0.0000 & 0.0000 & & \\
\hline \multirow{5}{*}{$\begin{array}{l}\text { I use nutrition } \\
\text { information to } \\
\text { diagnose health } \\
\text { conditions in } \\
\text { family members } \\
\text { and individuals. }\end{array}$} & Never & -0.7500 & 1.1991 & -3.1003 & 1.6003 & 0.39 & 0.5317 \\
\hline & Rarely & 0.0670 & 0.9510 & -1.7969 & 1.9308 & 0.00 & 0.9439 \\
\hline & Sometimes & -0.5594 & 0.9503 & -2.4219 & 1.3031 & 0.35 & 0.5561 \\
\hline & Often & -0.4261 & 0.9683 & -2.3239 & 1.4717 & 0.19 & 0.6599 \\
\hline & Always & 0.0000 & 0.0000 & 0.0000 & 0.0000 & & \\
\hline \multirow{2}{*}{ Major } & Dietetics & 0.0806 & 0.6140 & -1.1229 & 1.2841 & 0.02 & 0.8956 \\
\hline & Human Ntr & 0.0000 & 0.0000 & 0.0000 & 0.0000 & & \\
\hline
\end{tabular}


Table 77 (Part A): IRRITATING ROOMMATES/NEIGHBORS: Immerse yourself in your schoolwork.

\begin{tabular}{|c|c|c|c|c|c|c|c|}
\hline \multicolumn{8}{|c|}{ IRRITATING ROOMMATES/NEIGHBORS: Immerse yourself in your schoolwork. } \\
\hline \multirow{5}{*}{$\begin{array}{l}\text { Parameter } \\
\text { I only deliver } \\
\text { information } \\
\text { allowed at the } \\
\text { student level by the } \\
\text { CDR, as specified in } \\
\text { the Dietetic Scope } \\
\text { of Practice. }\end{array}$} & \multirow{2}{*}{$\begin{array}{l}\text { Response } \\
\text { Rarely }\end{array}$} & \multirow{2}{*}{$\begin{array}{r}\text { Estimate } \\
-0.1632\end{array}$} & \multirow{2}{*}{$\begin{array}{l}\begin{array}{l}\text { Standard } \\
\text { Error }\end{array} \\
2.3988\end{array}$} & \multicolumn{2}{|c|}{$\begin{array}{l}\text { Wald 95\% } \\
\text { Confidence Limits }\end{array}$} & \multirow{2}{*}{$\begin{array}{l}\text { Wald } \\
\text { Chi-Square } \\
0.00\end{array}$} & \multirow{2}{*}{\begin{tabular}{|l|}
$\begin{array}{l}\mathrm{Pr}> \\
\text { ChiSq }\end{array}$ \\
0.9458
\end{tabular}} \\
\hline & & & & -4.8647 & 4.5384 & & \\
\hline & Sometimes & -0.3894 & 0.8685 & -2.0916 & 1.3127 & 0.20 & 0.6539 \\
\hline & Often & -0.3659 & 0.8736 & -2.0781 & 1.3464 & 0.18 & 0.6754 \\
\hline & Always & 0.0000 & 0.0000 & 0.0000 & 0.0000 & & \\
\hline \multirow{5}{*}{$\begin{array}{l}\text { I comprehend the } \\
\text { scope of practice. }\end{array}$} & Never & 23.1930 & 76916.09 & -150730 & 150776.0 & 0.00 & 0.9998 \\
\hline & Rarely & -0.0246 & 1.4252 & -2.8179 & 2.7687 & 0.00 & 0.9862 \\
\hline & Sometimes & -0.8831 & 0.7941 & -2.4395 & 0.6733 & 1.24 & 0.2661 \\
\hline & Often & -0.8472 & 0.7187 & -2.2558 & 0.5614 & 1.39 & 0.2385 \\
\hline & Always & 0.0000 & 0.0000 & 0.0000 & 0.0000 & & \\
\hline \multirow{5}{*}{$\begin{array}{l}\text { I refer friends and } \\
\text { family to other } \\
\text { sources that are } \\
\text { outside my scope of } \\
\text { practice. }\end{array}$} & Never & 1.6953 & 1.8602 & -1.9506 & 5.3411 & 0.83 & 0.3621 \\
\hline & Rarely & 0.1090 & 2.8759 & -5.5277 & 5.7456 & 0.00 & 0.9698 \\
\hline & Sometimes & -0.5175 & 0.6705 & -1.8317 & 0.7967 & 0.60 & 0.4402 \\
\hline & Often & -0.2283 & 0.5607 & -1.3272 & 0.8707 & 0.17 & 0.6839 \\
\hline & Always & 0.0000 & 0.0000 & 0.0000 & 0.0000 & & \\
\hline \multirow{4}{*}{$\begin{array}{l}\text { I abide by the } \\
\text { Dietetics Code of } \\
\text { Ethics. }\end{array}$} & Rarely & 2.3356 & 2.6011 & -2.7624 & 7.4336 & 0.81 & 0.3692 \\
\hline & Sometimes & -0.4708 & 0.7180 & -1.8782 & 0.9365 & 0.43 & 0.5120 \\
\hline & Often & 0.3602 & 0.5705 & -0.7580 & 1.4785 & 0.40 & 0.5278 \\
\hline & Always & 0.0000 & 0.0000 & 0.0000 & 0.0000 & & \\
\hline \multirow{5}{*}{$\begin{array}{l}\text { I use nutrition } \\
\text { information to } \\
\text { diagnose health } \\
\text { conditions in family } \\
\text { members and } \\
\text { individuals. }\end{array}$} & Never & -1.8702 & 1.0819 & -3.9906 & 0.2503 & 2.99 & 0.0839 \\
\hline & Rarely & -1.5275 & 0.8382 & -3.1703 & 0.1153 & 3.32 & 0.0684 \\
\hline & Sometimes & -0.1788 & 0.8009 & -1.7486 & 1.3909 & 0.05 & 0.8233 \\
\hline & Often & -1.0458 & 0.8544 & -2.7205 & 0.6289 & 1.50 & 0.2210 \\
\hline & Always & 0.0000 & 0.0000 & 0.0000 & 0.0000 & & \\
\hline \multirow{2}{*}{ Major } & Dietetics & 0.1909 & 0.5013 & -0.7917 & 1.1734 & 0.14 & 0.7034 \\
\hline & Human Ntr & 0.0000 & 0.0000 & 0.0000 & 0.0000 & & \\
\hline
\end{tabular}


Table 77 (Part B): IRRITATING ROOMMATES/NEIGHBORS: Immerse yourself in your schoolwork.

\begin{tabular}{|l|r|r|}
\hline \multicolumn{3}{|c|}{ IRRITATING ROOMMATES/NEIGHBORS: Immerse yourself in your schoolwork. } \\
\hline Source & Chi-Square & Pr > ChiSq \\
\hline $\begin{array}{l}\text { I only deliver information allowed at the student level by the CDR, as } \\
\text { specified in the Dietetic Scope of Practice. }\end{array}$ & 0.22 & 0.9747 \\
\hline $\begin{array}{l}\text { I comprehend the scope of practice. } \\
\text { I refer friends and family to other sources that are outside my scope of } \\
\text { practice. }\end{array}$ & 1.65 & 0.79 \\
\hline $\begin{array}{l}\text { I abide by the Dietetics Code of Ethics. } \\
\text { I use nutrition information to diagnose health conditions in family } \\
\text { members and individuals. }\end{array}$ & 2.96 & 0.3983 \\
\hline Major & 8.70 & 0.0692 \\
\hline
\end{tabular}


Table 78: IRRITATING ROOMMATES/NEIGHBORS: Eat your favorite junk food.

\begin{tabular}{|c|c|c|c|c|c|c|c|}
\hline \multicolumn{8}{|c|}{ IRRITATING ROOMMATES/NEIGHBORS: Eat your favorite junk food. } \\
\hline \multirow{5}{*}{$\begin{array}{l}\text { Parameter } \\
\text { I only deliver } \\
\text { information } \\
\text { allowed at the } \\
\text { student level by } \\
\text { the CDR, as } \\
\text { specified in the } \\
\text { Dietetic Scope of } \\
\text { Practice. }\end{array}$} & \multirow{2}{*}{$\begin{array}{l}\text { Response } \\
\text { Rarely }\end{array}$} & \multirow{2}{*}{\begin{tabular}{|l|} 
Estimate \\
-25.6418
\end{tabular}} & \multirow{2}{*}{$\begin{array}{l}\text { Standard } \\
\text { Error }\end{array}$} & \multicolumn{2}{|c|}{$\begin{array}{l}\text { Wald 95\% } \\
\text { Confidence Limits }\end{array}$} & \multirow{2}{*}{$\begin{array}{l}\text { Wald } \\
\text { Chi-Square } \\
0.00\end{array}$} & \multirow{2}{*}{\begin{tabular}{|l|} 
Pr $>$ \\
ChiSq
\end{tabular}} \\
\hline & & & & -362977 & 362925.5 & & \\
\hline & Sometimes & 1.9072 & 1.0390 & -0.1292 & 3.9437 & 3.37 & 0.0664 \\
\hline & Often & 1.2109 & 1.0460 & -0.8392 & 3.2609 & 1.34 & 0.2470 \\
\hline & Always & 0.0000 & 0.0000 & 0.0000 & 0.0000 & & \\
\hline \multirow{5}{*}{$\begin{array}{l}\text { I comprehend the } \\
\text { scope of practice. }\end{array}$} & Never & 4.0501 & 1.9712 & 0.1866 & 7.9136 & 4.22 & 0.0399 \\
\hline & Rarely & 2.5417 & 1.5611 & -0.5180 & 5.6014 & 2.65 & 0.1035 \\
\hline & Sometimes & -0.5352 & 0.9663 & -2.4292 & 1.3587 & 0.31 & 0.5796 \\
\hline & Often & 0.7310 & 0.8708 & -0.9757 & 2.4377 & 0.70 & 0.4012 \\
\hline & Always & 0.0000 & 0.0000 & 0.0000 & 0.0000 & & \\
\hline \multirow{5}{*}{$\begin{array}{l}\text { I refer friends and } \\
\text { family to other } \\
\text { sources that are } \\
\text { outside my scope } \\
\text { of practice. }\end{array}$} & Never & -22.7267 & 261962.0 & -513459 & 513413.3 & 0.00 & 0.9999 \\
\hline & Rarely & 0.9584 & 2.0352 & -3.0306 & 4.9474 & 0.22 & 0.6377 \\
\hline & Sometimes & 1.1696 & 0.8053 & -0.4087 & 2.7480 & 2.11 & 0.1464 \\
\hline & Often & 1.1380 & 0.6593 & -0.1543 & 2.4303 & 2.98 & 0.0843 \\
\hline & Always & 0.0000 & 0.0000 & 0.0000 & 0.0000 & & \\
\hline \multirow{4}{*}{$\begin{array}{l}\text { I abide by the } \\
\text { Dietetics Code of } \\
\text { Ethics. }\end{array}$} & Rarely & -2.0047 & 240648.5 & -471664 & 471660.4 & 0.00 & 1.0000 \\
\hline & Sometimes & -1.2448 & 0.8659 & -2.9419 & 0.4523 & 2.07 & 0.1506 \\
\hline & Often & -1.3615 & 0.7157 & -2.7641 & 0.0412 & 3.62 & 0.0571 \\
\hline & Always & 0.0000 & 0.0000 & 0.0000 & 0.0000 & & \\
\hline \multirow{5}{*}{$\begin{array}{l}\text { I use nutrition } \\
\text { information to } \\
\text { diagnose health } \\
\text { conditions in } \\
\text { family members } \\
\text { and individuals. }\end{array}$} & Never & -2.8478 & 1.2555 & -5.3085 & -0.3871 & 5.15 & 0.0233 \\
\hline & Rarely & -1.8566 & 0.9542 & -3.7267 & 0.0135 & 3.79 & 0.0517 \\
\hline & Sometimes & -1.6399 & 0.9385 & -3.4792 & 0.1995 & 3.05 & 0.0806 \\
\hline & Often & -0.4158 & 0.9520 & -2.2816 & 1.4500 & 0.19 & 0.6623 \\
\hline & Always & 0.0000 & 0.0000 & 0.0000 & 0.0000 & & \\
\hline \multirow{2}{*}{ Major } & Dietetics & -0.1003 & 0.5455 & -1.1694 & 0.9688 & 0.03 & 0.8541 \\
\hline & Human Ntr & 0.0000 & 0.0000 & 0.0000 & 0.0000 & & \\
\hline
\end{tabular}


Table 79 (Part A): MIDTERMS/FINALS WEEK: Call a close family member or friend and talk about it.

\begin{tabular}{|c|c|c|c|c|c|c|c|}
\hline \multicolumn{8}{|c|}{ MIDTERMS/FINALS WEEK: Call a close family member or friend and talk about it. } \\
\hline \multirow{5}{*}{$\begin{array}{l}\text { Parameter } \\
\text { I only deliver } \\
\text { information allowed } \\
\text { at the student level } \\
\text { by the CDR, as } \\
\text { specified in the } \\
\text { Dietetic Scope of } \\
\text { Practice. }\end{array}$} & \multirow{2}{*}{$\begin{array}{l}\text { Response } \\
\text { Rarely }\end{array}$} & \multirow{2}{*}{\begin{tabular}{|l} 
Estimate \\
24.7906
\end{tabular}} & \multirow{2}{*}{\begin{tabular}{|l}
$\begin{array}{l}\text { Standard } \\
\text { Error }\end{array}$ \\
1.6275
\end{tabular}} & \multicolumn{2}{|c|}{$\begin{array}{l}\text { Wald 95\% } \\
\text { Confidence Limits }\end{array}$} & \multirow{2}{*}{\begin{tabular}{|r}
$\begin{array}{l}\text { Wald } \\
\text { Chi-Square }\end{array}$ \\
232.02
\end{tabular}} & \multirow{2}{*}{\begin{tabular}{|l|}
$\begin{array}{l}\mathrm{Pr}> \\
\text { ChiSq }\end{array}$ \\
$<.000$
\end{tabular}} \\
\hline & & & & 21.6007 & 27.9805 & & \\
\hline & Sometimes & 0.1179 & 0.8415 & -1.5315 & 1.7672 & 0.02 & 0.8886 \\
\hline & Often & 0.0847 & 0.8336 & -1.5491 & 1.7185 & 0.01 & 0.9191 \\
\hline & Always & 0.0000 & 0.0000 & 0.0000 & 0.0000 & & \\
\hline \multirow{5}{*}{$\begin{array}{l}\text { I comprehend the } \\
\text { scope of practice. }\end{array}$} & Never & -0.3590 & 1.7669 & -3.8220 & 3.1041 & 0.04 & 0.8390 \\
\hline & Rarely & 2.8739 & 1.3545 & 0.2191 & 5.5287 & 4.50 & 0.0339 \\
\hline & Sometimes & 0.8479 & 0.8222 & -0.7636 & 2.4594 & 1.06 & 0.3024 \\
\hline & Often & 0.3828 & 0.7012 & -0.9915 & 1.7572 & 0.30 & 0.5851 \\
\hline & Always & 0.0000 & 0.0000 & 0.0000 & 0.0000 & & \\
\hline \multirow{5}{*}{$\begin{array}{l}\text { I refer friends and } \\
\text { family to other } \\
\text { sources that are } \\
\text { outside my scope of } \\
\text { practice. }\end{array}$} & Never & -1.2588 & 1.8938 & -4.9707 & 2.4531 & 0.44 & 0.5063 \\
\hline & Rarely & 0.2872 & 1.6594 & -2.9652 & 3.5395 & 0.03 & 0.8626 \\
\hline & Sometimes & -0.9091 & 0.6973 & -2.2759 & 0.4576 & 1.70 & 0.1923 \\
\hline & Often & 0.0897 & 0.5830 & -1.0530 & 1.2324 & 0.02 & 0.8777 \\
\hline & Always & 0.0000 & 0.0000 & 0.0000 & 0.0000 & & \\
\hline \multirow{4}{*}{$\begin{array}{l}\text { I abide by the } \\
\text { Dietetics Code of } \\
\text { Ethics. }\end{array}$} & Rarely & -25.8356 & 0.0000 & -25.8356 & -25.8356 & & \\
\hline & Sometimes & -0.6834 & 0.7805 & -2.2132 & 0.8464 & 0.77 & 0.3813 \\
\hline & Often & 0.1819 & 0.6191 & -1.0315 & 1.3953 & 0.09 & 0.7689 \\
\hline & Always & 0.0000 & 0.0000 & 0.0000 & 0.0000 & & \\
\hline \multirow{5}{*}{$\begin{array}{l}\text { I use nutrition } \\
\text { information to } \\
\text { diagnose health } \\
\text { conditions in family } \\
\text { members and } \\
\text { individuals. }\end{array}$} & Never & -1.0205 & 1.0706 & -3.1187 & 1.0778 & 0.91 & 0.3405 \\
\hline & Rarely & -2.4847 & 0.8120 & -4.0763 & -0.8932 & 9.36 & 0.0022 \\
\hline & Sometimes & -1.4784 & 0.7710 & -2.9895 & 0.0326 & 3.68 & 0.0552 \\
\hline & Often & -1.0235 & 0.8843 & -2.7566 & 0.7096 & 1.34 & 0.2471 \\
\hline & Always & 0.0000 & 0.0000 & 0.0000 & 0.0000 & & \\
\hline \multirow{2}{*}{ Major } & Dietetics & 0.1568 & 0.5133 & -0.8492 & 1.1628 & 0.09 & 0.7600 \\
\hline & Human Ntr & 0.0000 & 0.0000 & 0.0000 & 0.0000 & & \\
\hline
\end{tabular}


Table 79 (Part B): MIDTERMS/FINALS WEEK: Call a close family member or friend and talk about it.

MIDTERMS/FINALS WEEK: Call a close family member or friend and talk about it.

\begin{tabular}{|l|r|r|}
\hline Source & Chi-Square & Pr > ChiSq \\
\hline $\begin{array}{l}\text { I only deliver information allowed at the student level by the CDR, as } \\
\text { specified in the Dietetic Scope of Practice. }\end{array}$ & 3.40 & 0.3345 \\
\hline I comprehend the scope of practice. & 5.37 & 0.2518 \\
\hline $\begin{array}{l}\text { I refer friends and family to other sources that are outside my scope of } \\
\text { practice. }\end{array}$ & 3.63 & 0.4588 \\
\hline I abide by the Dietetics Code of Ethics. & 5.78 & 0.1227 \\
\hline $\begin{array}{l}\text { I use nutrition information to diagnose health conditions in family } \\
\text { members and individuals. }\end{array}$ & 12.04 & 0.0170 \\
\hline Major & 0.09 & 0.7601 \\
\hline
\end{tabular}


Table 80 (Part A): MIDTERMS/FINALS WEEK: Cry.

\begin{tabular}{|c|c|c|c|c|c|c|c|}
\hline \multicolumn{8}{|c|}{ MIDTERMS/FINALS WEEK: Cry. } \\
\hline \multirow{5}{*}{$\begin{array}{l}\text { Parameter } \\
\text { I only deliver } \\
\text { information } \\
\text { allowed at the } \\
\text { student level by the } \\
\text { CDR, as specified in } \\
\text { the Dietetic Scope } \\
\text { of Practice. }\end{array}$} & \multirow{2}{*}{$\begin{array}{l}\text { Response } \\
\text { Rarely }\end{array}$} & \multirow{2}{*}{$\begin{array}{r}\text { Estimate } \\
0.1652\end{array}$} & \multirow{2}{*}{$\begin{array}{l}\begin{array}{l}\text { Standard } \\
\text { Error }\end{array} \\
1.7997\end{array}$} & \multicolumn{2}{|c|}{$\begin{array}{l}\text { Wald 95\% } \\
\text { Confidence Limits }\end{array}$} & \multirow{2}{*}{$\begin{array}{l}\text { Wald } \\
\text { Chi-Square } \\
0.01\end{array}$} & \multirow{2}{*}{ 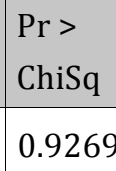 } \\
\hline & & & & -3.3622 & 3.6925 & & \\
\hline & Sometimes & -0.7850 & 0.9377 & -2.6230 & 1.0529 & 0.70 & 0.4025 \\
\hline & Often & -0.8190 & 0.9523 & -2.6855 & 1.0476 & 0.74 & 0.3898 \\
\hline & Always & 0.0000 & 0.0000 & 0.0000 & 0.0000 & & \\
\hline \multirow{5}{*}{$\begin{array}{l}\text { I comprehend the } \\
\text { scope of practice. }\end{array}$} & Never & -22.1743 & 95055.47 & -186327 & 186283.1 & 0.00 & 0.9998 \\
\hline & Rarely & 0.9887 & 1.3033 & -1.5658 & 3.5432 & 0.58 & 0.4481 \\
\hline & Sometimes & -0.0290 & 0.8887 & -1.7708 & 1.7129 & 0.00 & 0.9740 \\
\hline & Often & 0.3689 & 0.7938 & -1.1869 & 1.9247 & 0.22 & 0.6421 \\
\hline & Always & 0.0000 & 0.0000 & 0.0000 & 0.0000 & & \\
\hline \multirow{5}{*}{$\begin{array}{l}\text { I refer friends and } \\
\text { family to other } \\
\text { sources that are } \\
\text { outside my scope of } \\
\text { practice. }\end{array}$} & Never & -22.9942 & 95055.47 & -186328 & 186282.3 & 0.00 & 0.9998 \\
\hline & Rarely & 2.2234 & 1.6382 & -0.9874 & 5.4343 & 1.84 & 0.1747 \\
\hline & Sometimes & 0.1774 & 0.6489 & -1.0945 & 1.4493 & 0.07 & 0.7846 \\
\hline & Often & 0.2192 & 0.5703 & -0.8987 & 1.3370 & 0.15 & 0.7007 \\
\hline & Always & 0.0000 & 0.0000 & 0.0000 & 0.0000 & & \\
\hline \multirow{4}{*}{$\begin{array}{l}\text { I abide by the } \\
\text { Dietetics Code of } \\
\text { Ethics. }\end{array}$} & Rarely & 0.4271 & 2.1372 & -3.7616 & 4.6159 & 0.04 & 0.8416 \\
\hline & Sometimes & 0.3556 & 0.7872 & -1.1873 & 1.8985 & 0.20 & 0.6514 \\
\hline & Often & 0.4618 & 0.6565 & -0.8248 & 1.7485 & 0.49 & 0.4817 \\
\hline & Always & 0.0000 & 0.0000 & 0.0000 & 0.0000 & & \\
\hline \multirow{5}{*}{$\begin{array}{l}\text { I use nutrition } \\
\text { information to } \\
\text { diagnose health } \\
\text { conditions in family } \\
\text { members and } \\
\text { individuals. }\end{array}$} & Never & 0.8179 & 1.1082 & -1.3541 & 2.9898 & 0.54 & 0.4605 \\
\hline & Rarely & 1.1460 & 0.9477 & -0.7115 & 3.0036 & 1.46 & 0.2266 \\
\hline & Sometimes & 0.5756 & 0.9553 & -1.2968 & 2.4480 & 0.36 & 0.5468 \\
\hline & Often & 1.6399 & 1.0281 & -0.3750 & 3.6549 & 2.54 & 0.1107 \\
\hline & Always & 0.0000 & 0.0000 & 0.0000 & 0.0000 & & \\
\hline \multirow{2}{*}{ Major } & Dietetics & 0.0229 & 0.5306 & -1.0171 & 1.0629 & 0.00 & 0.9656 \\
\hline & Human Ntr & 0.0000 & 0.0000 & 0.0000 & 0.0000 & & \\
\hline
\end{tabular}


Table 80 (Part B): MIDTERMS/FINALS WEEK: Cry.

\begin{tabular}{|l|r|r|}
\hline \multicolumn{3}{|c|}{ MIDTERMS/FINALS WEEK: Cry. } \\
\hline Source & Chi-Square & Pr > ChiSq \\
\hline $\begin{array}{l}\text { I only deliver information allowed at the student level by the CDR, as } \\
\text { specified in the Dietetic Scope of Practice. }\end{array}$ & 1.05 & 0.7882 \\
\hline I comprehend the scope of practice. & 2.09 & 0.7199 \\
\hline $\begin{array}{l}\text { I refer friends and family to other sources that are outside my scope of } \\
\text { practice. }\end{array}$ & 3.53 & 0.4733 \\
\hline I abide by the Dietetics Code of Ethics. & 0.52 & 0.9154 \\
\hline $\begin{array}{l}\text { I use nutrition information to diagnose health conditions in family } \\
\text { members and individuals. }\end{array}$ & 4.89 & 0.2988 \\
\hline Major & 0.00 & 0.9656 \\
\hline
\end{tabular}


Table 81 (Part A): MIDTERMS/FINALS WEEK: Go to the gym or exercise outdoors.

\begin{tabular}{|c|c|c|c|c|c|c|c|}
\hline \multicolumn{8}{|c|}{ MIDTERMS/FINALS WEEK: Go to the gym or exercise outdoors. } \\
\hline \multirow{5}{*}{$\begin{array}{l}\text { Parameter } \\
\text { I only deliver } \\
\text { information } \\
\text { allowed at the } \\
\text { student level by } \\
\text { the CDR, as } \\
\text { specified in the } \\
\text { Dietetic Scope of } \\
\text { Practice. }\end{array}$} & \multirow{2}{*}{$\begin{array}{l}\text { Response } \\
\text { Rarely }\end{array}$} & \multirow{2}{*}{$\begin{array}{r}\text { Estimate } \\
4.6350\end{array}$} & \multirow{2}{*}{$\begin{array}{l}\text { Standard } \\
\text { Error } \\
2.0766\end{array}$} & \multicolumn{2}{|c|}{$\begin{array}{l}\text { Wald 95\% } \\
\text { Confidence Limits }\end{array}$} & \multirow{2}{*}{$\begin{array}{l}\text { Wald } \\
\text { Chi-Square } \\
4.98\end{array}$} & \multirow{2}{*}{\begin{tabular}{|l}
$\begin{array}{l}\mathrm{Pr}> \\
\mathrm{ChiSq}\end{array}$ \\
0.0256
\end{tabular}} \\
\hline & & & & 0.5649 & 8.7051 & & \\
\hline & Sometimes & 2.7899 & 0.8845 & 1.0563 & 4.5234 & 9.95 & 0.0016 \\
\hline & Often & 2.5728 & 0.8848 & 0.8386 & 4.3069 & 8.46 & 0.0036 \\
\hline & Always & 0.0000 & 0.0000 & 0.0000 & 0.0000 & & \\
\hline \multirow{5}{*}{$\begin{array}{l}\text { I comprehend the } \\
\text { scope of practice. }\end{array}$} & Never & -2.1837 & 1.7651 & -5.6431 & 1.2758 & 1.53 & 0.2160 \\
\hline & Rarely & 0.1034 & 1.2969 & -2.4384 & 2.6452 & 0.01 & 0.9364 \\
\hline & Sometimes & -0.8830 & 0.7762 & -2.4042 & 0.6383 & 1.29 & 0.2553 \\
\hline & Often & -0.4248 & 0.6939 & -1.7848 & 0.9352 & 0.37 & 0.5404 \\
\hline & Always & 0.0000 & 0.0000 & 0.0000 & 0.0000 & & \\
\hline \multirow{5}{*}{$\begin{array}{l}\text { I refer friends and } \\
\text { family to other } \\
\text { sources that are } \\
\text { outside my scope } \\
\text { of practice. }\end{array}$} & Never & -1.5462 & 1.8315 & -5.1359 & 2.0434 & 0.71 & 0.3985 \\
\hline & Rarely & -27.3468 & 79282.69 & -155419 & 155363.9 & 0.00 & 0.9997 \\
\hline & Sometimes & -1.9420 & 0.7500 & -3.4120 & -0.4720 & 6.70 & 0.0096 \\
\hline & Often & -1.4453 & 0.6330 & -2.6860 & -0.2046 & 5.21 & 0.0224 \\
\hline & Always & 0.0000 & 0.0000 & 0.0000 & 0.0000 & & \\
\hline \multirow{4}{*}{$\begin{array}{l}\text { I abide by the } \\
\text { Dietetics Code of } \\
\text { Ethics. }\end{array}$} & Rarely & -2.0644 & 2.0721 & -6.1258 & 1.9969 & 0.99 & 0.3191 \\
\hline & Sometimes & -1.2067 & 0.7650 & -2.7060 & 0.2926 & 2.49 & 0.1147 \\
\hline & Often & -0.7801 & 0.5913 & -1.9391 & 0.3788 & 1.74 & 0.1871 \\
\hline & Always & 0.0000 & 0.0000 & 0.0000 & 0.0000 & & \\
\hline \multirow{5}{*}{$\begin{array}{l}\text { I use nutrition } \\
\text { information to } \\
\text { diagnose health } \\
\text { conditions in } \\
\text { family members } \\
\text { and individuals. }\end{array}$} & Never & -1.0825 & 0.9596 & -2.9633 & 0.7983 & 1.27 & 0.2593 \\
\hline & Rarely & -0.8362 & 0.7723 & -2.3498 & 0.6775 & 1.17 & 0.2789 \\
\hline & Sometimes & -0.4217 & 0.7837 & -1.9577 & 1.1143 & 0.29 & 0.5905 \\
\hline & Often & -0.9332 & 0.8396 & -2.5788 & 0.7125 & 1.24 & 0.2664 \\
\hline & Always & 0.0000 & 0.0000 & 0.0000 & 0.0000 & & \\
\hline \multirow{2}{*}{ Major } & Dietetics & 0.8404 & 0.5118 & -0.1627 & 1.8434 & 2.70 & 0.1006 \\
\hline & Human Ntr & 0.0000 & 0.0000 & 0.0000 & 0.0000 & & \\
\hline
\end{tabular}


Table 81 (Part B): MIDTERMS/FINALS WEEK: Go to the gym or exercise outdoors.

\begin{tabular}{|l|r|r|}
\hline \multicolumn{3}{|c|}{ MIDTERMS/FINALS WEEK: Go to the gym or exercise outdoors. } \\
\hline Source & Chi-Square & Pr > ChiSq \\
\hline $\begin{array}{l}\text { I only deliver information allowed at the student level by the CDR, as } \\
\text { specified in the Dietetic Scope of Practice. }\end{array}$ & 11.97 & 0.0075 \\
\hline I comprehend the scope of practice. & 2.87 & 0.5806 \\
\hline $\begin{array}{l}\text { I refer friends and family to other sources that are outside my scope of } \\
\text { practice. }\end{array}$ & 15.93 & 0.0031 \\
\hline I abide by the Dietetics Code of Ethics. & 3.13 & 0.3726 \\
\hline $\begin{array}{l}\text { I use nutrition information to diagnose health conditions in family } \\
\text { members and individuals. }\end{array}$ & 2.23 & 0.6944 \\
\hline Major & 2.73 & 0.0982 \\
\hline
\end{tabular}


Table 82: MIDTERMS/FINALS WEEK: Drink alcoholic beverages.

\begin{tabular}{|c|c|c|c|c|c|c|c|}
\hline \multicolumn{8}{|c|}{ MIDTERMS/FINALS WEEK: Drink alcoholic beverages. } \\
\hline \multirow{5}{*}{$\begin{array}{l}\text { Parameter } \\
\text { I only deliver } \\
\text { information } \\
\text { allowed at the } \\
\text { student level by the } \\
\text { CDR, as specified in } \\
\text { the Dietetic Scope } \\
\text { of Practice. }\end{array}$} & \multirow{2}{*}{$\begin{array}{l}\text { Response } \\
\text { Rarely }\end{array}$} & \multirow{2}{*}{\begin{tabular}{|r|} 
Estimate \\
23.7987
\end{tabular}} & \multirow{2}{*}{\begin{tabular}{|l|}
$\begin{array}{l}\text { Standard } \\
\text { Error }\end{array}$ \\
279289.6
\end{tabular}} & \multicolumn{2}{|c|}{$\begin{array}{l}\text { Wald 95\% } \\
\text { Confidence Limits }\end{array}$} & \multirow{2}{*}{$\begin{array}{l}\text { Wald } \\
\text { Chi-Square } \\
0.00\end{array}$} & \multirow{2}{*}{\begin{tabular}{|l}
$\begin{array}{l}\mathrm{Pr}> \\
\text { ChiSq }\end{array}$ \\
0.9990
\end{tabular}} \\
\hline & & & & -547374 & 547421.4 & & \\
\hline & Sometimes & 0.7564 & 1.0359 & -1.2740 & 2.7868 & 0.53 & 0.4653 \\
\hline & Often & 0.3414 & 1.0781 & -1.7716 & 2.4545 & 0.10 & 0.7515 \\
\hline & Always & 0.0000 & 0.0000 & 0.0000 & 0.0000 & & \\
\hline \multirow{5}{*}{$\begin{array}{l}\text { I comprehend the } \\
\text { scope of practice. }\end{array}$} & Never & -24.2256 & 323453.2 & -633981 & 633932.4 & 0.00 & 0.9999 \\
\hline & Rarely & -46.5569 & 175760.9 & -344532 & 344438.5 & 0.00 & 0.9998 \\
\hline & Sometimes & 0.7700 & 1.0783 & -1.3434 & 2.8834 & 0.51 & 0.4752 \\
\hline & Often & 1.3798 & 0.9743 & -0.5297 & 3.2893 & 2.01 & 0.1567 \\
\hline & Always & 0.0000 & 0.0000 & 0.0000 & 0.0000 & & \\
\hline \multirow{5}{*}{$\begin{array}{l}\text { I refer friends and } \\
\text { family to other } \\
\text { sources that are } \\
\text { outside my scope of } \\
\text { practice. }\end{array}$} & Never & -25.0121 & 323453.2 & -633982 & 633931.6 & 0.00 & 0.9999 \\
\hline & Rarely & -22.8825 & 183334.3 & -359351 & 359305.6 & 0.00 & 0.9999 \\
\hline & Sometimes & -0.4525 & 0.8439 & -2.1065 & 1.2015 & 0.29 & 0.5918 \\
\hline & Often & 0.2404 & 0.6773 & -1.0870 & 1.5679 & 0.13 & 0.7226 \\
\hline & Always & 0.0000 & 0.0000 & 0.0000 & 0.0000 & & \\
\hline \multirow{4}{*}{$\begin{array}{l}\text { I abide by the } \\
\text { Dietetics Code of } \\
\text { Ethics. }\end{array}$} & Rarely & 2.1774 & 250544.5 & -491056 & 491060.5 & 0.00 & 1.0000 \\
\hline & Sometimes & -1.0160 & 0.9892 & -2.9548 & 0.9227 & 1.06 & 0.3044 \\
\hline & Often & 0.1493 & 0.7254 & -1.2724 & 1.5710 & 0.04 & 0.8369 \\
\hline & Always & 0.0000 & 0.0000 & 0.0000 & 0.0000 & & \\
\hline \multirow{5}{*}{$\begin{array}{l}\text { I use nutrition } \\
\text { information to } \\
\text { diagnose health } \\
\text { conditions in family } \\
\text { members and } \\
\text { individuals. }\end{array}$} & Never & 0.2091 & 1.2203 & -2.1826 & 2.6008 & 0.03 & 0.8639 \\
\hline & Rarely & 0.4164 & 1.0361 & -1.6144 & 2.4472 & 0.16 & 0.6878 \\
\hline & Sometimes & 0.2963 & 0.9924 & -1.6488 & 2.2414 & 0.09 & 0.7653 \\
\hline & Often & 0.3803 & 1.0341 & -1.6466 & 2.4072 & 0.14 & 0.7131 \\
\hline & Always & 0.0000 & 0.0000 & 0.0000 & 0.0000 & & \\
\hline \multirow{2}{*}{ Major } & Dietetics & 0.2055 & 0.6045 & -0.9794 & 1.3904 & 0.12 & 0.7339 \\
\hline & Human Ntr & 0.0000 & 0.0000 & 0.0000 & 0.0000 & & \\
\hline
\end{tabular}


Table 83 (Part A): MIDTERMS/FINALS WEEK: Immerse yourself in your schoolwork.

\begin{tabular}{|c|c|c|c|c|c|c|c|}
\hline \multicolumn{8}{|c|}{ MIDTERMS/FINALS WEEK: Immerse yourself in your schoolwork. } \\
\hline \multirow{5}{*}{$\begin{array}{l}\text { Parameter } \\
\text { I only deliver } \\
\text { information } \\
\text { allowed at the } \\
\text { student level by } \\
\text { the CDR, as } \\
\text { specified in the } \\
\text { Dietetic Scope of } \\
\text { Practice. }\end{array}$} & \multirow{2}{*}{$\begin{array}{l}\text { Response } \\
\text { Rarely }\end{array}$} & \multirow{2}{*}{$\begin{array}{r}\text { Estimate } \\
25.6622\end{array}$} & \multirow{2}{*}{$\begin{array}{l}\begin{array}{l}\text { Standard } \\
\text { Error }\end{array} \\
1.6060\end{array}$} & \multicolumn{2}{|c|}{$\begin{array}{l}\text { Wald 95\% } \\
\text { Confidence Limits }\end{array}$} & \multirow{2}{*}{\begin{tabular}{|r|}
$\begin{array}{l}\text { Wald } \\
\text { Chi-Square }\end{array}$ \\
255.34
\end{tabular}} & \multirow{2}{*}{\begin{tabular}{|l} 
Pr $>$ \\
ChiSq
\end{tabular}} \\
\hline & & & & 22.5145 & 28.8098 & & \\
\hline & Sometimes & 1.2403 & 1.0053 & -0.7301 & 3.2107 & 1.52 & 0.2173 \\
\hline & Often & 2.0407 & 1.0762 & -0.0686 & 4.1501 & 3.60 & 0.0579 \\
\hline & Always & 0.0000 & 0.0000 & 0.0000 & 0.0000 & & \\
\hline \multirow{5}{*}{$\begin{array}{l}\text { I comprehend the } \\
\text { scope of practice. }\end{array}$} & Never & 21.6416 & 171947.6 & -336989 & 337032.8 & 0.00 & 0.9999 \\
\hline & Rarely & -0.0367 & 1.3823 & -2.7460 & 2.6726 & 0.00 & 0.9788 \\
\hline & Sometimes & -0.4738 & 0.9840 & -2.4024 & 1.4548 & 0.23 & 0.6301 \\
\hline & Often & -1.1390 & 0.9345 & -2.9707 & 0.6926 & 1.49 & 0.2229 \\
\hline & Always & 0.0000 & 0.0000 & 0.0000 & 0.0000 & & \\
\hline \multirow{5}{*}{$\begin{array}{l}\text { I refer friends and } \\
\text { family to other } \\
\text { sources that are } \\
\text { outside my scope } \\
\text { of practice. }\end{array}$} & Never & 24.5545 & 171947.6 & -336987 & 337035.7 & 0.00 & 0.9999 \\
\hline & Rarely & -0.7463 & 1.7627 & -4.2013 & 2.7086 & 0.18 & 0.6720 \\
\hline & Sometimes & -1.2463 & 0.7817 & -2.7784 & 0.2857 & 2.54 & 0.1108 \\
\hline & Often & -0.7686 & 0.6651 & -2.0721 & 0.5349 & 1.34 & 0.2478 \\
\hline & Always & 0.0000 & 0.0000 & 0.0000 & 0.0000 & & \\
\hline \multirow{4}{*}{$\begin{array}{l}\text { I abide by the } \\
\text { Dietetics Code of } \\
\text { Ethics. }\end{array}$} & Rarely & -26.4454 & 0.0000 & -26.4454 & -26.4454 & & \\
\hline & Sometimes & -1.5528 & 0.9616 & -3.4375 & 0.3318 & 2.61 & 0.1063 \\
\hline & Often & -1.5624 & 0.7923 & -3.1154 & -0.0094 & 3.89 & 0.0486 \\
\hline & Always & 0.0000 & 0.0000 & 0.0000 & 0.0000 & & \\
\hline \multirow{5}{*}{$\begin{array}{l}\text { I use nutrition } \\
\text { information to } \\
\text { diagnose health } \\
\text { conditions in } \\
\text { family members } \\
\text { and individuals. }\end{array}$} & Never & -0.9036 & 1.1449 & -3.1474 & 1.3403 & 0.62 & 0.4300 \\
\hline & Rarely & -0.6964 & 0.8417 & -2.3462 & 0.9534 & 0.68 & 0.4081 \\
\hline & Sometimes & 8769 & 0.9209 & -0.9280 & 2.6818 & 0.91 & 0.3410 \\
\hline & Often & -0.0665 & 0.9320 & -1.8932 & 1.7602 & 0.01 & 0.9431 \\
\hline & Always & 0.0000 & 0.0000 & 0.0000 & 0.0000 & & \\
\hline \multirow{2}{*}{ Major } & Dietetics & -1.0609 & 0.5667 & -2.1716 & 0.0498 & 3.50 & 0.0612 \\
\hline & Human Ntr & 0.0000 & 0.0000 & 0.0000 & 0.0000 & & \\
\hline
\end{tabular}


Table 83 (Part B): MIDTERMS/FINALS WEEK: Immerse yourself in your schoolwork.

\begin{tabular}{|l|r|r|}
\hline \multicolumn{3}{|c|}{ MIDTERMS/FINALS WEEK: Immerse yourself in your schoolwork. } \\
\hline Source & Chi-Square & Pr > ChiSq \\
\hline $\begin{array}{l}\text { I only deliver information allowed at the student level by the CDR, as } \\
\text { specified in the Dietetic Scope of Practice. }\end{array}$ & 6.55 & 0.0877 \\
\hline $\begin{array}{l}\text { I comprehend the scope of practice. } \\
\text { I refer friends and family to other sources that are outside my scope of } \\
\text { practice. }\end{array}$ & 2.94 & 0.5687 \\
\hline $\begin{array}{l}\text { I abide by the Dietetics Code of Ethics. } \\
\text { I use nutrition information to diagnose health conditions in family } \\
\text { members and individuals. }\end{array}$ & 7.89 & 0.2907 \\
\hline Major & 7.20 & 0.0483 \\
\hline
\end{tabular}


Table 84 (Part A): MIDTERMS/FINALS WEEK: Eat your favorite junk food.

\begin{tabular}{|c|c|c|c|c|c|c|c|}
\hline \multicolumn{8}{|c|}{ MIDTERMS/FINALS WEEK: Eat your favorite junk food. } \\
\hline \multirow{5}{*}{$\begin{array}{l}\text { Parameter } \\
\text { I only deliver } \\
\text { information } \\
\text { allowed at the } \\
\text { student level by the } \\
\text { CDR, as specified in } \\
\text { the Dietetic Scope } \\
\text { of Practice. }\end{array}$} & \multirow{2}{*}{$\begin{array}{l}\text { Response } \\
\text { Rarely }\end{array}$} & \multirow{2}{*}{$\begin{array}{r}\text { Estimate } \\
0.7599\end{array}$} & \multirow{2}{*}{\begin{tabular}{|l|l}
$\begin{array}{l}\text { Standard } \\
\text { Error }\end{array}$ \\
1.8911
\end{tabular}} & \multicolumn{2}{|c|}{$\begin{array}{l}\text { Wald 95\% } \\
\text { Confidence Limits }\end{array}$} & \multirow{2}{*}{\begin{tabular}{|r}
$\begin{array}{l}\text { Wald } \\
\text { Chi-Square }\end{array}$ \\
0.16
\end{tabular}} & \multirow{2}{*}{ 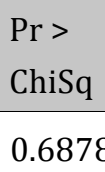 } \\
\hline & & & & -2.9466 & 4.4664 & & \\
\hline & Sometimes & 0.6697 & 0.8609 & -1.0177 & 2.3570 & 0.61 & 0.4366 \\
\hline & Often & 0.9502 & 0.8466 & -0.7091 & 2.6096 & 1.26 & 0.2617 \\
\hline & Always & 0.0000 & 0.0000 & 0.0000 & 0.0000 & & \\
\hline \multirow{5}{*}{$\begin{array}{l}\text { I comprehend the } \\
\text { scope of practice. }\end{array}$} & Never & 26.7268 & 98685.06 & -193392 & 193445.9 & 0.00 & 0.9998 \\
\hline & Rarely & 0.7203 & 1.2524 & -1.7344 & 3.1751 & 0.33 & 0.5652 \\
\hline & Sometimes & -0.3897 & 0.8222 & -2.0012 & 1.2218 & 0.22 & 0.6355 \\
\hline & Often & 0.2470 & 0.7387 & -1.2007 & 1.6948 & 0.11 & 0.7380 \\
\hline & Always & 0.0000 & 0.0000 & 0.0000 & 0.0000 & & \\
\hline \multirow{5}{*}{$\begin{array}{l}\text { I refer friends and } \\
\text { family to other } \\
\text { sources that are } \\
\text { outside my scope of } \\
\text { practice. }\end{array}$} & Never & 3.3573 & 1.9121 & -0.3903 & 7.1050 & 3.08 & 0.0791 \\
\hline & Rarely & 0.7142 & 1.6447 & -2.5093 & 3.9378 & 0.19 & 0.6641 \\
\hline & Sometimes & 1.0238 & 0.7012 & -0.3505 & 2.3981 & 2.13 & 0.1443 \\
\hline & Often & 0.8836 & 0.5824 & -0.2578 & 2.0251 & 2.30 & 0.1292 \\
\hline & Always & 0.0000 & 0.0000 & 0.0000 & 0.0000 & & \\
\hline \multirow{4}{*}{$\begin{array}{l}\text { I abide by the } \\
\text { Dietetics Code of } \\
\text { Ethics. }\end{array}$} & Rarely & -2.5973 & 2.1973 & -6.9039 & 1.7094 & 1.40 & 0.2372 \\
\hline & Sometimes & -1.0893 & 0.7921 & -2.6418 & 0.4633 & 1.89 & 0.1691 \\
\hline & Often & -1.2099 & 0.6256 & -2.4360 & 0.0163 & 3.74 & 0.0531 \\
\hline & Always & 0.0000 & 0.0000 & 0.0000 & 0.0000 & & \\
\hline \multirow{5}{*}{$\begin{array}{l}\text { I use nutrition } \\
\text { information to } \\
\text { diagnose health } \\
\text { conditions in family } \\
\text { members and } \\
\text { individuals. }\end{array}$} & Never & -1.6678 & 1.1247 & -3.8723 & 0.5366 & 2.20 & 0.1381 \\
\hline & Rarely & 0.2906 & 0.8225 & -1.3216 & 1.9027 & 0.12 & 0.7239 \\
\hline & Sometimes & -0.7643 & 0.8520 & -2.4342 & 0.9055 & 0.80 & 0.3697 \\
\hline & Often & 0.6848 & 0.8917 & -1.0628 & 2.4325 & 0.59 & 0.4425 \\
\hline & Always & 0.0000 & 0.0000 & 0.0000 & 0.0000 & & \\
\hline \multirow{2}{*}{ Major } & Dietetics & -0.9298 & 0.5319 & -1.9723 & 0.1128 & 3.06 & 0.0805 \\
\hline & Human Ntr & 0.0000 & 0.0000 & 0.0000 & 0.0000 & & \\
\hline
\end{tabular}


Table 84 (Part B): MIDTERMS/FINALS WEEK: Eat your favorite junk food.

\begin{tabular}{|l|r|r|}
\hline \multicolumn{3}{|c|}{ MIDTERMS/FINALS WEEK: Eat your favorite junk food. } \\
\hline Source & Chi-Square & Pr > ChiSq \\
\hline $\begin{array}{l}\text { I only deliver information allowed at the student level by the CDR, as } \\
\text { specified in the Dietetic Scope of Practice. }\end{array}$ & 1.33 & 0.7230 \\
\hline I comprehend the scope of practice. & 8.74 & 0.0678 \\
\hline $\begin{array}{l}\text { I refer friends and family to other sources that are outside my scope of } \\
\text { practice. }\end{array}$ & 4.88 & 0.2994 \\
\hline $\begin{array}{l}\text { I abide by the Dietetics Code of Ethics. } \\
\text { I use nutrition information to diagnose health conditions in family } \\
\text { members and individuals. }\end{array}$ & 4.95 & 0.1755 \\
\hline Major & 3.11 & 0.32 \\
\hline
\end{tabular}


Table 85 (Part A): MODELING BEHAVIORS: I use information from the dietary guidelines to model nutrition behaviors.

\begin{tabular}{|c|c|c|c|c|c|c|c|}
\hline \multicolumn{8}{|c|}{$\begin{array}{c}\text { MODELING BEHAVIORS: I use information from the dietary guidelines to model nutrition } \\
\text { behaviors. }\end{array}$} \\
\hline \multirow{5}{*}{$\begin{array}{l}\text { Parameter } \\
\text { I only deliver } \\
\text { information allowed at } \\
\text { the student level by the } \\
\text { CDR, as specified in the } \\
\text { Dietetic Scope of } \\
\text { Practice. }\end{array}$} & \multirow{2}{*}{\begin{tabular}{|l} 
Response \\
Disagree
\end{tabular}} & \multirow{2}{*}{$\begin{array}{r}\text { Estimate } \\
-2.7392\end{array}$} & \multirow{2}{*}{\begin{tabular}{|l|}
$\begin{array}{l}\text { Standard } \\
\text { Error }\end{array}$ \\
2.2488
\end{tabular}} & \multicolumn{2}{|c|}{$\begin{array}{l}\text { Wald 95\% } \\
\text { Confidence Limits }\end{array}$} & \multirow{2}{*}{\begin{tabular}{|r|}
$\begin{array}{l}\text { Wald } \\
\text { Chi-Square }\end{array}$ \\
1.48
\end{tabular}} & \multirow{2}{*}{$\begin{array}{l}\text { Pr }> \\
\text { ChiSq } \\
0.2232\end{array}$} \\
\hline & & & & -7.1469 & 1.6684 & & \\
\hline & Neutral & 1.3491 & 0.9687 & -0.5495 & 3.2476 & 1.94 & 0.1637 \\
\hline & Agree & 0.9036 & 0.9541 & -0.9663 & 2.7736 & 0.90 & 0.3436 \\
\hline & $\begin{array}{l}\text { Strongly } \\
\text { Agree }\end{array}$ & 0.0000 & 0.0000 & 0.0000 & 0.0000 & & \\
\hline \multirow{5}{*}{$\begin{array}{l}\text { I comprehend the } \\
\text { scope of practice. }\end{array}$} & $\begin{array}{l}\text { Strongly } \\
\text { Disagree }\end{array}$ & -2.3438 & 2.3060 & -6.8635 & 2.1760 & 1.03 & 0.3095 \\
\hline & Disagree & -0.3718 & 1.5219 & -3.3547 & 2.6111 & 0.06 & 0.8070 \\
\hline & Neutral & -1.4679 & 0.9445 & -3.3190 & 0.3833 & 2.42 & 0.1201 \\
\hline & Agree & -0.3038 & 0.8258 & -1.9223 & 1.3146 & 0.14 & 0.7129 \\
\hline & $\begin{array}{l}\text { Strongly } \\
\text { Agree }\end{array}$ & 0.0000 & 0.0000 & 0.0000 & 0.0000 & & \\
\hline \multirow{5}{*}{$\begin{array}{l}\text { I refer friends and } \\
\text { family to other sources } \\
\text { that are outside my } \\
\text { scope of practice. }\end{array}$} & $\begin{array}{l}\text { Strongly } \\
\text { Disagree }\end{array}$ & 0.7389 & 2.3141 & -3.7966 & 5.2743 & 0.10 & 0.7495 \\
\hline & Disagree & -3.3185 & 1.9045 & -7.0512 & 0.4142 & 3.04 & 0.0814 \\
\hline & Neutral & -0.4207 & 0.7275 & -1.8465 & 1.0051 & 0.33 & 0.5631 \\
\hline & Agree & -0.3521 & 0.6339 & -1.5945 & 0.8902 & 0.31 & 0.5785 \\
\hline & $\begin{array}{l}\text { Strongly } \\
\text { Agree }\end{array}$ & 0.0000 & 0.0000 & 0.0000 & 0.0000 & & \\
\hline \multirow{4}{*}{$\begin{array}{l}\text { I abide by the Dietetics } \\
\text { Code of Ethics. }\end{array}$} & Disagree & 0.2010 & 2.4105 & -4.5236 & 4.9255 & 0.01 & 0.9336 \\
\hline & Neutral & -1.8858 & 0.8704 & -3.5917 & -0.1799 & 4.69 & 0.0303 \\
\hline & Agree & -1.6433 & 0.6981 & -3.0114 & -0.2751 & 5.54 & 0.0186 \\
\hline & $\begin{array}{l}\text { Strongly } \\
\text { Agree }\end{array}$ & 0.0000 & 0.0000 & 0.0000 & 0.0000 & & \\
\hline \multirow{5}{*}{$\begin{array}{l}\text { I use nutrition } \\
\text { information to } \\
\text { diagnose health } \\
\text { conditions in family } \\
\text { members and } \\
\text { individuals. }\end{array}$} & $\begin{array}{l}\text { Strongly } \\
\text { Disagree }\end{array}$ & -2.3590 & 1.1229 & -4.5599 & -0.1581 & 4.41 & 0.0357 \\
\hline & Disagree & -0.1886 & 0.9031 & -1.9586 & 1.5814 & 0.04 & 0.8346 \\
\hline & Neutral & -1.3168 & 0.9091 & -3.0985 & 0.4650 & 2.10 & 0.1475 \\
\hline & Agree & -0.6518 & 0.9867 & -2.5858 & 1.2822 & 0.44 & 0.5089 \\
\hline & $\begin{array}{l}\text { Strongly } \\
\text { Agree }\end{array}$ & 0.0000 & 0.0000 & 0.0000 & 0.0000 & & \\
\hline \multirow{2}{*}{ Major } & Dietetics & 1.0252 & 0.5837 & -0.1188 & 2.1693 & 3.08 & 0.0790 \\
\hline & Human Ntr & 0.0000 & 0.0000 & 0.0000 & 0.0000 & & \\
\hline
\end{tabular}


Table 85 (Part B): MODELING BEHAVIORS: I use information from the dietary guidelines to model nutrition behaviors.

\begin{tabular}{|c|c|c|}
\hline \multicolumn{3}{|c|}{$\begin{array}{c}\text { MODELING BEHAVIORS: I use information from the dietary guidelines to model nutrition } \\
\text { behaviors. }\end{array}$} \\
\hline Source & Chi-Square & $\operatorname{Pr}>$ ChiSq \\
\hline $\begin{array}{l}\text { I only deliver information allowed at the student level by the CDR, as } \\
\text { specified in the Dietetic Scope of Practice. }\end{array}$ & 5.15 & 0.1610 \\
\hline I comprehend the scope of practice. & 4.74 & 0.3155 \\
\hline $\begin{array}{l}\text { I refer friends and family to other sources that are outside my scope of } \\
\text { practice. }\end{array}$ & 3.31 & 0.5072 \\
\hline I abide by the Dietetics Code of Ethics. & 6.66 & 0.0835 \\
\hline $\begin{array}{l}\text { I use nutrition information to diagnose health conditions in family } \\
\text { members and individuals. }\end{array}$ & 8.05 & 0.0899 \\
\hline Major & 3.19 & 0.0742 \\
\hline
\end{tabular}


Table 86 (Part A): MODELING BEHAVIORS: I advise friends and family to use the dietary guidelines to make food and nutrition decisions.

\begin{tabular}{|c|c|c|c|c|c|c|c|}
\hline \multirow{5}{*}{$\begin{array}{l}\text { Parameter } \\
\text { I only deliver } \\
\text { information } \\
\text { allowed at the } \\
\text { student level by the } \\
\text { CDR, as specified in } \\
\text { the Dietetic Scope } \\
\text { of Practice. }\end{array}$} & \multirow{2}{*}{\begin{tabular}{|l} 
Response \\
Disagree
\end{tabular}} & \multirow{2}{*}{\begin{tabular}{|r|} 
Estimate \\
-0.3331
\end{tabular}} & \multirow{2}{*}{\begin{tabular}{|l|}
$\begin{array}{l}\text { Standard } \\
\text { Error }\end{array}$ \\
2.2221
\end{tabular}} & \multicolumn{2}{|c|}{$\begin{array}{l}\text { Wald } 95 \% \\
\text { Confidence Limits }\end{array}$} & \multirow{2}{*}{$\begin{array}{l}\text { Wald } \\
\text { Chi-Square } \\
0.02\end{array}$} & \multirow{2}{*}{$\begin{array}{l}\text { Pr }> \\
\text { ChiSq } \\
0.8809\end{array}$} \\
\hline & & & & -4.6883 & 4.0222 & & \\
\hline & Neutral & 0.9255 & 0.9582 & -0.9526 & 2.8035 & 0.93 & 0.3341 \\
\hline & Agree & 0.4835 & 0.9889 & -1.4547 & 2.4218 & 0.24 & 0.6249 \\
\hline & $\begin{array}{l}\text { Strongly } \\
\text { Agree }\end{array}$ & 0.0000 & 0.0000 & 0.0000 & 0.0000 & & \\
\hline \multirow{5}{*}{$\begin{array}{l}\text { I comprehend the } \\
\text { scope of practice. }\end{array}$} & $\begin{array}{l}\text { Strongly } \\
\text { Disagree }\end{array}$ & 23.1982 & 100657.3 & -197261 & 197307.9 & 0.00 & 0.9998 \\
\hline & Disagree & 2.0065 & 1.4551 & -0.8455 & 4.8584 & 1.90 & 0.1679 \\
\hline & Neutral & -0.1648 & 0.8917 & -1.9126 & 1.5830 & 0.03 & 0.8534 \\
\hline & Agree & -0.0323 & 0.7897 & -1.5801 & 1.5155 & 0.00 & 0.9674 \\
\hline & \begin{tabular}{|l|} 
Strongly \\
Agree
\end{tabular} & 0.0000 & 0.0000 & 0.0000 & 0.0000 & & \\
\hline \multirow{5}{*}{$\begin{array}{l}\text { I refer friends and } \\
\text { family to other } \\
\text { sources that are } \\
\text { outside my scope } \\
\text { of practice. }\end{array}$} & $\begin{array}{l}\text { Strongly } \\
\text { Disagree }\end{array}$ & -1.1189 & 2.1794 & -5.3905 & 3.1527 & 0.26 & 0.6077 \\
\hline & Disagree & -3.8633 & 1.6915 & -7.1786 & -0.5480 & 5.22 & 0.0224 \\
\hline & Neutral & -1.6171 & 0.7567 & -3.1002 & -0.1340 & 4.57 & 0.0326 \\
\hline & Agree & -1.5323 & 0.6737 & -2.8527 & -0.2118 & 5.17 & 0.0229 \\
\hline & \begin{tabular}{|l|} 
Strongly \\
Agree
\end{tabular} & 0.0000 & 0.0000 & 0.0000 & 0.0000 & & \\
\hline \multirow{4}{*}{$\begin{array}{l}\text { I abide by the } \\
\text { Dietetics Code of } \\
\text { Ethics. }\end{array}$} & Disagree & 1.2463 & 2.5689 & -3.7887 & 6.2814 & 0.24 & 0.6276 \\
\hline & Neutral & -1.3224 & 0.8685 & -3.0246 & 0.3798 & 2.32 & 0.1278 \\
\hline & Agree & -1.3489 & 0.7265 & -2.7727 & 0.0750 & 3.45 & 0.0633 \\
\hline & \begin{tabular}{|l|} 
Strongly \\
Agree
\end{tabular} & 0.0000 & 0.0000 & 0.0000 & 0.0000 & & \\
\hline \multirow{5}{*}{$\begin{array}{l}\text { I use nutrition } \\
\text { information to } \\
\text { diagnose health } \\
\text { conditions in } \\
\text { family members } \\
\text { and individuals. }\end{array}$} & \begin{tabular}{|l} 
Strongly \\
Disagree
\end{tabular} & -0.2423 & 1.1612 & -2.5183 & 2.0337 & 0.04 & 0.8347 \\
\hline & Disagree & -0.4781 & 0.9465 & -2.3331 & 1.3770 & 0.26 & 0.6135 \\
\hline & Neutral & -0.1666 & 0.9373 & -2.0037 & 1.6705 & 0.03 & 0.8589 \\
\hline & Agree & 0.1278 & 1.0140 & -1.8597 & 2.1153 & 0.02 & 0.8997 \\
\hline & \begin{tabular}{|l|} 
Strongly \\
Agree
\end{tabular} & 0.0000 & 0.0000 & 0.0000 & 0.0000 & & \\
\hline \multirow{2}{*}{ Major } & Dietetics & 0.5273 & 0.5500 & -0.5507 & 1.6053 & 0.92 & 0.3377 \\
\hline & Human Ntr & 0.0000 & 0.0000 & 0.0000 & 0.0000 & & \\
\hline
\end{tabular}


Table 86 (Part B): MODELING BEHAVIORS: I advise friends and family to use the dietary guidelines to make food and nutrition decisions.

\begin{tabular}{|c|c|c|}
\hline Source & Chi-Square & Pr $>$ ChiSq \\
\hline $\begin{array}{l}\text { I only deliver information allowed at the student level by the CDR, as } \\
\text { specified in the Dietetic Scope of Practice. }\end{array}$ & 1.44 & 0.6966 \\
\hline I comprehend the scope of practice. & 4.61 & 0.3292 \\
\hline $\begin{array}{l}\text { I refer friends and family to other sources that are outside my scope of } \\
\text { practice. }\end{array}$ & 8.33 & 0.0801 \\
\hline I abide by the Dietetics Code of Ethics. & 4.38 & 0.2234 \\
\hline $\begin{array}{l}\text { I use nutrition information to diagnose health conditions in family } \\
\text { members and individuals. }\end{array}$ & 0.94 & 0.9193 \\
\hline Major & 0.92 & 0.3383 \\
\hline
\end{tabular}


Table 87 (Part A): MODELING BEHAVIORS: I use correct portion sizes when eating with friends and family.

\begin{tabular}{|c|c|c|c|c|c|c|c|}
\hline \multicolumn{8}{|c|}{ MODELING BEHAVIORS: I use correct portion sizes when eating with friends and family. } \\
\hline \multirow{5}{*}{$\begin{array}{l}\text { Parameter } \\
\text { I only deliver } \\
\text { information allowed } \\
\text { at the student level } \\
\text { by the CDR, as } \\
\text { specified in the } \\
\text { Dietetic Scope of } \\
\text { Practice. }\end{array}$} & \multirow{2}{*}{\begin{tabular}{|l} 
Response \\
Disagree \\
\end{tabular}} & \multirow{2}{*}{\begin{tabular}{|r} 
Estimate \\
-0.1916
\end{tabular}} & \multirow{2}{*}{$\begin{array}{l}\text { Standard } \\
\text { Error } \\
2.0878\end{array}$} & \multicolumn{2}{|c|}{$\begin{array}{l}\text { Wald 95\% } \\
\text { Confidence Limits }\end{array}$} & \multirow{2}{*}{\begin{tabular}{|r} 
Wald \\
Chi-Square \\
0.01 \\
\end{tabular}} & \multirow{2}{*}{$\begin{array}{l}\operatorname{Pr}> \\
\text { ChiSq } \\
0.9269\end{array}$} \\
\hline & & & & -4.2836 & 3.9003 & & \\
\hline & Neutral & 1.1817 & 0.9157 & -0.6129 & 2.9764 & 1.67 & 0.1969 \\
\hline & Agree & 0.0938 & 0.8982 & -1.6666 & 1.8543 & 0.01 & 0.9168 \\
\hline & $\begin{array}{l}\text { Strongly } \\
\text { Agree }\end{array}$ & 0.0000 & 0.0000 & 0.0000 & 0.0000 & & \\
\hline \multirow{5}{*}{$\begin{array}{l}\text { I comprehend the } \\
\text { scope of practice. }\end{array}$} & $\begin{array}{l}\text { Strongly } \\
\text { Disagree }\end{array}$ & -1.6589 & 1.7739 & -5.1358 & 1.8179 & 0.87 & 0.3497 \\
\hline & Disagree & 0.5714 & 1.5721 & -2.5098 & 3.6525 & 0.13 & 0.7163 \\
\hline & Neutral & -0.2029 & 0.8497 & -1.8684 & 1.4625 & 0.06 & 0.8113 \\
\hline & Agree & 0.2243 & 0.7695 & -1.2838 & 1.7325 & 0.08 & 0.7707 \\
\hline & $\begin{array}{l}\text { Strongly } \\
\text { Agree }\end{array}$ & 0.0000 & 0.0000 & 0.0000 & 0.0000 & & \\
\hline \multirow{5}{*}{$\begin{array}{l}\text { I refer friends and } \\
\text { family to other } \\
\text { sources that are } \\
\text { outside my scope of } \\
\text { practice. }\end{array}$} & $\begin{array}{l}\text { Strongly } \\
\text { Disagree }\end{array}$ & -4.9346 & 1.9330 & -8.7233 & -1.1460 & 6.52 & 0.0107 \\
\hline & Disagree & -4.2647 & 2.7542 & -9.6627 & 1.1334 & 2.40 & 0.1215 \\
\hline & Neutral & -3.1040 & 0.7438 & -4.5618 & -1.6463 & 17.42 & $<.0001$ \\
\hline & Agree & -2.5081 & 0.6469 & -3.7761 & -1.2401 & 15.03 & 0.0001 \\
\hline & $\begin{array}{l}\text { Strongly } \\
\text { Agree }\end{array}$ & 0.0000 & 0.0000 & 0.0000 & 0.0000 & & \\
\hline \multirow{4}{*}{$\begin{array}{l}\text { I abide by the } \\
\text { Dietetics Code of } \\
\text { Ethics. }\end{array}$} & Disagree & 1.0095 & 2.0618 & -3.0316 & 5.0506 & 0.24 & 0.6244 \\
\hline & Neutral & -0.0612 & 0.7522 & -1.5356 & 1.4132 & 0.01 & 0.9352 \\
\hline & Agree & 0.7731 & 0.6094 & -0.4213 & 1.9674 & 1.61 & 0.2046 \\
\hline & $\begin{array}{l}\text { Strongly } \\
\text { Agree }\end{array}$ & 0.0000 & 0.0000 & 0.0000 & 0.0000 & & \\
\hline \multirow{5}{*}{$\begin{array}{l}\text { I use nutrition } \\
\text { information to } \\
\text { diagnose health } \\
\text { conditions in family } \\
\text { members and } \\
\text { individuals. }\end{array}$} & $\begin{array}{l}\text { Strongly } \\
\text { Disagree }\end{array}$ & -0.1792 & 1.1194 & -2.3733 & 2.0149 & 0.03 & 0.8728 \\
\hline & Disagree & -1.5463 & 0.8654 & -3.2424 & 0.1498 & 3.19 & 0.0740 \\
\hline & Neutral & -1.0373 & 0.8353 & -2.6744 & 0.5999 & 1.54 & 0.2143 \\
\hline & Agree & -1.5286 & 0.9150 & -3.3220 & 0.2648 & 2.79 & 0.0948 \\
\hline & $\begin{array}{l}\text { Strongly } \\
\text { Agree }\end{array}$ & 0.0000 & 0.0000 & 0.0000 & 0.0000 & & \\
\hline \multirow{2}{*}{ Major } & Dietetics & 2.6696 & 0.5991 & 1.4953 & 3.8439 & 19.85 & $<.0001$ \\
\hline & Human Ntr & 0.0000 & 0.0000 & 0.0000 & 0.0000 & & \\
\hline
\end{tabular}


Table 87 (Part B): MODELING BEHAVIORS: I use correct portion sizes when eating with friends and family.

MODELING BEHAVIORS: I use correct portion sizes when eating with friends and family.

\begin{tabular}{|l|r|r|}
\hline Source & Chi-Square & Pr > ChiSq \\
\hline $\begin{array}{l}\text { I only deliver information allowed at the student level by the CDR, as } \\
\text { specified in the Dietetic Scope of Practice. }\end{array}$ & 4.43 & 0.2187 \\
\hline I comprehend the scope of practice. & 1.77 & 0.7788 \\
\hline $\begin{array}{l}\text { I refer friends and family to other sources that are outside my scope of } \\
\text { practice. }\end{array}$ & 24.52 & $<.0001$ \\
\hline I abide by the Dietetics Code of Ethics. & 3.16 & 0.3680 \\
\hline $\begin{array}{l}\text { I use nutrition information to diagnose health conditions in family } \\
\text { members and individuals. }\end{array}$ & 5.16 & 0.2712 \\
\hline Major & 22.43 & $<.0001$ \\
\hline
\end{tabular}


Table 88 (Part A): MODELING BEHAVIORS: When grocery shopping with friends and family, I encourage them to read the nutrition facts labels.

\begin{tabular}{|c|c|c|c|c|c|c|c|}
\hline \multicolumn{8}{|c|}{$\begin{array}{l}\text { MODELING BEHAVIORS: When grocery shopping with friends and family, I encourage them to } \\
\text { read the nutrition facts labels. }\end{array}$} \\
\hline \multirow{5}{*}{$\begin{array}{l}\text { Parameter } \\
\text { I only deliver } \\
\text { information allowed } \\
\text { at the student level } \\
\text { by the CDR, as } \\
\text { specified in the } \\
\text { Dietetic Scope of } \\
\text { Practice. }\end{array}$} & \multirow{2}{*}{\begin{tabular}{|l} 
Response \\
Disagree
\end{tabular}} & \multirow{2}{*}{$\begin{array}{r}\text { Estimate } \\
-0.2601\end{array}$} & \multirow{2}{*}{\begin{tabular}{|l|}
$\begin{array}{l}\text { Standard } \\
\text { Error }\end{array}$ \\
2.0619
\end{tabular}} & \multicolumn{2}{|c|}{$\begin{array}{l}\text { Wald 95\% } \\
\text { Confidence Limits }\end{array}$} & \multirow{2}{*}{\begin{tabular}{|r|}
$\begin{array}{l}\text { Wald } \\
\text { Chi-Square }\end{array}$ \\
0.02
\end{tabular}} & \multirow{2}{*}{\begin{tabular}{|l|}
$\begin{array}{l}\text { Pr }> \\
\text { ChiSq }\end{array}$ \\
0.8996
\end{tabular}} \\
\hline & & & & -4.3014 & 3.7812 & & \\
\hline & Neutral & 0.6446 & 0.9663 & -1.2493 & 2.5384 & 0.45 & 0.5047 \\
\hline & Agree & 0.2645 & 1.0126 & -1.7201 & 2.2491 & 0.07 & 0.7939 \\
\hline & $\begin{array}{l}\text { Strongly } \\
\text { Agree }\end{array}$ & 0.0000 & 0.0000 & 0.0000 & 0.0000 & & \\
\hline \multirow{5}{*}{$\begin{array}{l}\text { I comprehend the } \\
\text { scope of practice. }\end{array}$} & $\begin{array}{l}\text { Strongly } \\
\text { Disagree }\end{array}$ & -3.5030 & 1.9537 & -7.3321 & 0.3261 & 3.21 & 0.0730 \\
\hline & \begin{tabular}{|l|} 
Disagree \\
\end{tabular} & -2.3411 & 1.6211 & -5.5183 & 0.8362 & 2.09 & 0.1487 \\
\hline & Neutral & -2.7762 & 1.0263 & -4.7877 & -0.7646 & 7.32 & 0.0068 \\
\hline & Agree & -2.3545 & 0.9574 & -4.2311 & -0.4780 & 6.05 & 0.0139 \\
\hline & $\begin{array}{l}\text { Strongly } \\
\text { Agree }\end{array}$ & 0.0000 & 0.0000 & 0.0000 & 0.0000 & & \\
\hline \multirow{5}{*}{$\begin{array}{l}\text { I refer friends and } \\
\text { family to other } \\
\text { sources that are } \\
\text { outside my scope of } \\
\text { practice. }\end{array}$} & $\begin{array}{l}\text { Strongly } \\
\text { Disagree }\end{array}$ & 0.4941 & 1.8970 & -3.2239 & 4.2121 & 0.07 & 0.7945 \\
\hline & Disagree & -4.1629 & 2.3828 & -8.8330 & 0.5072 & 3.05 & 0.0806 \\
\hline & Neutral & -0.8664 & 0.6985 & -2.2355 & 0.5026 & 1.54 & 0.2148 \\
\hline & Agree & -1.0658 & 0.6147 & -2.2705 & 0.1390 & 3.01 & 0.0829 \\
\hline & \begin{tabular}{|l|} 
Strongly \\
Agree
\end{tabular} & 0.0000 & 0.0000 & 0.0000 & 0.0000 & & \\
\hline \multirow{4}{*}{$\begin{array}{l}\text { I abide by the } \\
\text { Dietetics Code of } \\
\text { Ethics. }\end{array}$} & Disagree & 2.3885 & 2.0903 & -1.7083 & 6.4854 & 1.31 & 0.2532 \\
\hline & Neutral & -1.1372 & 0.8113 & -2.7273 & 0.4529 & 1.96 & 0.1610 \\
\hline & Agree & -0.4223 & 0.6946 & -1.7837 & 0.9391 & 0.37 & 0.5432 \\
\hline & \begin{tabular}{|l|l|} 
Strongly \\
Agree
\end{tabular} & 0.0000 & 0.0000 & 0.0000 & 0.0000 & & \\
\hline \multirow{5}{*}{$\begin{array}{l}\text { I use nutrition } \\
\text { information to } \\
\text { diagnose health } \\
\text { conditions in family } \\
\text { members and } \\
\text { individuals. }\end{array}$} & $\begin{array}{l}\text { Strongly } \\
\text { Disagree }\end{array}$ & -0.3837 & 1.0567 & -2.4549 & 1.6874 & 0.13 & 0.7165 \\
\hline & Disagree & 1.2009 & 0.9060 & -0.5749 & 2.9768 & 1.76 & 0.1850 \\
\hline & \begin{tabular}{|l|} 
Neutral \\
\end{tabular} & 1.5221 & 0.9214 & -0.2838 & 3.3281 & 2.73 & 0.0985 \\
\hline & Agree & 1.8723 & 0.9627 & -0.0145 & 3.7590 & 3.78 & 0.0518 \\
\hline & \begin{tabular}{|l|} 
Strongly \\
Agree
\end{tabular} & 0.0000 & 0.0000 & 0.0000 & 0.0000 & & \\
\hline \multirow{2}{*}{ Major } & Dietetics & 0.5902 & 0.5224 & -0.4337 & 1.6140 & 1.28 & 0.2586 \\
\hline & Human Ntr & 0.0000 & 0.0000 & 0.0000 & 0.0000 & & \\
\hline
\end{tabular}


Table 88 (Part B): MODELING BEHAVIORS: When grocery shopping with friends and family, I encourage them to read the nutrition facts labels.

MODELING BEHAVIORS: When grocery shopping with friends and family, I encourage them to read the nutrition facts labels.

\begin{tabular}{|l|r|r|}
\hline Source & Chi-Square & Pr > ChiSq \\
\hline $\begin{array}{l}\text { I only deliver information allowed at the student level by the CDR, as } \\
\text { specified in the Dietetic Scope of Practice. }\end{array}$ & 0.87 & 0.8315 \\
\hline I comprehend the scope of practice. & 9.56 & 0.0486 \\
\hline $\begin{array}{l}\text { I refer friends and family to other sources that are outside my scope of } \\
\text { practice. }\end{array}$ & 6.13 & 0.1896 \\
\hline I abide by the Dietetics Code of Ethics. & 4.56 & 0.2066 \\
\hline $\begin{array}{l}\text { I use nutrition information to diagnose health conditions in family } \\
\text { members and individuals. }\end{array}$ & 8.76 & 0.0675 \\
\hline Major & 1.28 & 0.2587 \\
\hline
\end{tabular}


Table 89 (Part A): MODELING BEHAVIORS: I follow dietary guidelines in my alcohol consumption.

\begin{tabular}{|c|c|c|c|c|c|c|c|}
\hline \multicolumn{8}{|c|}{ MODELING BEHAVIORS: I follow dietary guidelines in my alcohol consumption. } \\
\hline \multirow{5}{*}{$\begin{array}{l}\text { Parameter } \\
\text { I only deliver } \\
\text { information allowed at } \\
\text { the student level by } \\
\text { the CDR, as specified } \\
\text { in the Dietetic Scope of } \\
\text { Practice. }\end{array}$} & \multirow{2}{*}{\begin{tabular}{|l} 
Response \\
Disagree
\end{tabular}} & \multirow{2}{*}{\begin{tabular}{|r} 
Estimate \\
-1.1775
\end{tabular}} & \multirow{2}{*}{$\begin{array}{l}\text { Standard } \\
\text { Error } \\
1.6920\end{array}$} & \multicolumn{2}{|c|}{$\begin{array}{l}\text { Wald 95\% } \\
\text { Confidence Limits }\end{array}$} & \multirow{2}{*}{ 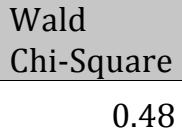 } & \multirow{2}{*}{$\begin{array}{l}\text { Pr > } \\
\text { ChiSq } \\
0.4865\end{array}$} \\
\hline & & & & -4.4937 & 2.1387 & & \\
\hline & Neutral & -0.2892 & 0.8901 & -2.0338 & 1.4555 & 0.11 & 0.7453 \\
\hline & Agree & -0.5716 & 0.8986 & -2.3329 & 1.1897 & 0.40 & 0.5247 \\
\hline & $\begin{array}{l}\text { Strongly } \\
\text { Agree }\end{array}$ & 0.0000 & 0.0000 & 0.0000 & 0.0000 & & \\
\hline \multirow{5}{*}{$\begin{array}{l}\text { I comprehend the } \\
\text { scope of practice. }\end{array}$} & $\begin{array}{l}\text { Strongly } \\
\text { Disagree }\end{array}$ & 0.6544 & 1.6602 & -2.5996 & 3.9083 & 0.16 & 0.6935 \\
\hline & Disagree & 1.9258 & 1.2073 & -0.4403 & 4.2920 & 2.54 & 0.1107 \\
\hline & Neutral & 0.7667 & 0.7979 & -0.7971 & 2.3306 & 0.92 & 0.3366 \\
\hline & Agree & 1.1233 & 0.7502 & -0.3471 & 2.5938 & 2.24 & 0.1343 \\
\hline & $\begin{array}{l}\text { Strongly } \\
\text { Agree }\end{array}$ & 0.0000 & 0.0000 & 0.0000 & 0.0000 & & \\
\hline \multirow{5}{*}{$\begin{array}{l}\text { I refer friends and } \\
\text { family to other } \\
\text { sources that are } \\
\text { outside my scope of } \\
\text { practice. }\end{array}$} & $\begin{array}{l}\text { Strongly } \\
\text { Disagree }\end{array}$ & -0.6844 & 1.7522 & -4.1186 & 2.7498 & 0.15 & 0.6961 \\
\hline & Disagree & -0.3093 & 1.4557 & -3.1624 & 2.5437 & 0.05 & 0.8317 \\
\hline & Neutral & -0.9563 & 0.6816 & -2.2922 & 0.3796 & 1.97 & 0.1606 \\
\hline & Agree & -0.7459 & 0.5964 & -1.9148 & 0.4229 & 1.56 & 0.2110 \\
\hline & $\begin{array}{l}\text { Strongly } \\
\text { Agree }\end{array}$ & 0.0000 & 0.0000 & 0.0000 & 0.0000 & & \\
\hline \multirow{4}{*}{$\begin{array}{l}\text { I abide by the Dietetics } \\
\text { Code of Ethics. }\end{array}$} & Disagree & -2.3136 & 1.9136 & -6.0642 & 1.4370 & 1.46 & 0.2266 \\
\hline & Neutral & -0.3930 & 0.7142 & -1.7929 & 1.0069 & 0.30 & 0.5822 \\
\hline & Agree & -0.0668 & 0.5974 & -1.2377 & 1.1040 & 0.01 & 0.9109 \\
\hline & $\begin{array}{l}\text { Strongly } \\
\text { Agree }\end{array}$ & 0.0000 & 0.0000 & 0.0000 & 0.0000 & & \\
\hline \multirow{5}{*}{$\begin{array}{l}\text { I use nutrition } \\
\text { information to } \\
\text { diagnose health } \\
\text { conditions in family } \\
\text { members and } \\
\text { individuals. }\end{array}$} & $\begin{array}{l}\text { Strongly } \\
\text { Disagree }\end{array}$ & -1.9759 & 1.0407 & -4.0157 & 0.0638 & 3.60 & 0.0576 \\
\hline & Disagree & -2.2492 & 0.8642 & -3.9430 & -0.5554 & 6.77 & 0.0092 \\
\hline & Neutral & -0.7395 & 0.8316 & -2.3695 & 0.8905 & 0.79 & 0.3739 \\
\hline & Agree & -1.3708 & 0.9002 & -3.1351 & 0.3935 & 2.32 & 0.1278 \\
\hline & $\begin{array}{l}\text { Strongly } \\
\text { Agree }\end{array}$ & 0.0000 & 0.0000 & 0.0000 & 0.0000 & & \\
\hline \multirow{2}{*}{ Major } & Dietetics & 0.1572 & 0.5243 & -0.8704 & 1.1848 & 0.09 & 0.7643 \\
\hline & Human Ntr & 0.0000 & 0.0000 & 0.0000 & 0.0000 & & \\
\hline
\end{tabular}


Table 89 (Part B): MODELING BEHAVIORS: I follow dietary guidelines in my alcohol consumption.

\begin{tabular}{|l|r|r|}
\hline \multicolumn{3}{|c|}{ MODELING BEHAVIORS: I follow dietary guidelines in my alcohol consumption. } \\
\hline Source & Chi-Square & Pr > ChiSq \\
\hline $\begin{array}{l}\text { I only deliver information allowed at the student level by the CDR, as } \\
\text { specified in the Dietetic Scope of Practice. }\end{array}$ & 0.84 & 0.8404 \\
\hline I comprehend the scope of practice. & 3.45 & 0.4852 \\
\hline $\begin{array}{l}\text { I refer friends and family to other sources that are outside my scope of } \\
\text { practice. }\end{array}$ & 2.37 & 0.6685 \\
\hline I abide by the Dietetics Code of Ethics. & 1.69 & 0.6399 \\
\hline $\begin{array}{l}\text { I use nutrition information to diagnose health conditions in family } \\
\text { members and individuals. }\end{array}$ & 11.46 & 0.0219 \\
\hline Major & 0.09 & 0.7640 \\
\hline
\end{tabular}


Table 90 (Part A): CREDIBILITY AND RELIABILITY BEHAVIORS: The information I give to others is usable, practical, and accurate.

\begin{tabular}{|c|c|c|c|c|c|c|c|}
\hline \multicolumn{8}{|c|}{$\begin{array}{l}\text { CREDIBILITY AND RELIABILITY BEHAVIORS: The information I give to others is usable, practical, } \\
\text { and accurate. }\end{array}$} \\
\hline \multirow{5}{*}{$\begin{array}{l}\text { Parameter } \\
\text { I only deliver } \\
\text { information } \\
\text { allowed at the } \\
\text { student level by the } \\
\text { CDR, as specified in } \\
\text { the Dietetic Scope } \\
\text { of Practice. }\end{array}$} & \multirow{2}{*}{\begin{tabular}{|l} 
Response \\
Disagree
\end{tabular}} & \multirow{2}{*}{\begin{tabular}{|r} 
Estimate \\
0.1250
\end{tabular}} & \multirow{2}{*}{\begin{tabular}{|r|}
$\begin{array}{l}\text { Standard } \\
\text { Error }\end{array}$ \\
2.5134
\end{tabular}} & \multicolumn{2}{|c|}{$\begin{array}{l}\text { Wald } 95 \% \\
\text { Confidence Limits }\end{array}$} & \multirow{2}{*}{$\begin{array}{l}\text { Wald } \\
\text { Chi-Square } \\
\quad 0.00\end{array}$} & \multirow{2}{*}{$\begin{array}{l}\begin{array}{l}\operatorname{Pr}> \\
\text { ChiSq }\end{array} \\
0.9603\end{array}$} \\
\hline & & & & -4.8012 & 5.0513 & & \\
\hline & Neutral & -0.5955 & 1.0637 & -2.6803 & 1.4892 & 0.31 & 0.5756 \\
\hline & Agree & 0.2353 & 1.1082 & -1.9368 & 2.4073 & 0.05 & 0.8319 \\
\hline & $\begin{array}{l}\text { Strongly } \\
\text { Agree }\end{array}$ & 0.0000 & 0.0000 & 0.0000 & 0.0000 & & \\
\hline \multirow{5}{*}{$\begin{array}{l}\text { I comprehend the } \\
\text { scope of practice. }\end{array}$} & $\begin{array}{l}\text { Strongly } \\
\text { Disagree }\end{array}$ & -29.8607 & 124189.1 & -243436 & 243376.3 & 0.00 & 0.9998 \\
\hline & Disagree & -3.2312 & 1.9271 & -7.0082 & 0.5459 & 2.81 & 0.0936 \\
\hline & Neutral & -1.0012 & 1.1272 & -3.2105 & 1.2081 & 0.79 & 0.3744 \\
\hline & Agree & -1.5174 & 1.0217 & -3.5199 & 0.4851 & 2.21 & 0.1375 \\
\hline & $\begin{array}{l}\text { Strongly } \\
\text { Agree }\end{array}$ & 0.0000 & 0.0000 & 0.0000 & 0.0000 & & \\
\hline \multirow{5}{*}{$\begin{array}{l}\text { I refer friends and } \\
\text { family to other } \\
\text { sources that are } \\
\text { outside my scope of } \\
\text { practice. }\end{array}$} & $\begin{array}{l}\text { Strongly } \\
\text { Disagree }\end{array}$ & -4.8726 & 3.7094 & -12.1429 & 2.3976 & 1.73 & 0.1890 \\
\hline & Disagree & 0.4617 & 2.1163 & -3.6861 & 4.6095 & 0.05 & 0.8273 \\
\hline & Neutral & -2.7997 & 0.9689 & -4.6988 & -0.9006 & 8.35 & 0.0039 \\
\hline & Agree & -1.9768 & 0.7715 & -3.4889 & -0.4647 & 6.57 & 0.0104 \\
\hline & $\begin{array}{l}\text { Strongly } \\
\text { Agree }\end{array}$ & 0.0000 & 0.0000 & 0.0000 & 0.0000 & & \\
\hline \multirow{4}{*}{$\begin{array}{l}\text { I abide by the } \\
\text { Dietetics Code of } \\
\text { Ethics. }\end{array}$} & Disagree & -0.4163 & 3.0649 & -6.4235 & 5.5909 & 0.02 & 0.8920 \\
\hline & Neutral & -1.0737 & 1.0926 & -3.2152 & 1.0679 & 0.97 & 0.3258 \\
\hline & Agree & -0.1723 & 0.8020 & -1.7441 & 1.3995 & 0.05 & 0.8299 \\
\hline & $\begin{array}{l}\text { Strongly } \\
\text { Agree }\end{array}$ & 0.0000 & 0.0000 & 0.0000 & 0.0000 & & \\
\hline \multirow{5}{*}{$\begin{array}{l}\text { I use nutrition } \\
\text { information to } \\
\text { diagnose health } \\
\text { conditions in family } \\
\text { members and } \\
\text { individuals. }\end{array}$} & $\begin{array}{l}\text { Strongly } \\
\text { Disagree }\end{array}$ & 0.7716 & 1.3311 & -1.8374 & 3.3805 & 0.34 & 0.5622 \\
\hline & Disagree & -0.5126 & 1.1309 & -2.7292 & 1.7039 & 0.21 & 0.6503 \\
\hline & Neutral & 0.3356 & 1.1398 & -1.8983 & 2.5695 & 0.09 & 0.7684 \\
\hline & Agree & 0.2900 & 1.2309 & -2.1226 & 2.7026 & 0.06 & 0.8137 \\
\hline & $\begin{array}{l}\text { Strongly } \\
\text { Agree }\end{array}$ & 0.0000 & 0.0000 & 0.0000 & 0.0000 & & \\
\hline \multirow{2}{*}{ Major } & Dietetics & 1.0068 & 0.7378 & -0.4392 & 2.4529 & 1.86 & 0.1723 \\
\hline & Human Ntr & 0.0000 & 0.0000 & 0.0000 & 0.0000 & & \\
\hline
\end{tabular}


Table 90 (Part B): CREDIBILITY AND RELIABILITY BEHAVIORS: The information I give to others is usable, practical, and accurate.

\begin{tabular}{|l|r|r|}
\hline $\begin{array}{l}\text { CREDIBILITY AND RELIABILITY BEHAVIORS: The information I give to others is usable, practical, } \\
\text { and accurate. }\end{array}$ & Chi-Square & Pr > ChiSq \\
\hline Source & 1.37 & 0.7132 \\
\hline $\begin{array}{l}\text { I only deliver information allowed at the student level by the CDR, as } \\
\text { specified in the Dietetic Scope of Practice. }\end{array}$ & 13.37 & 0.0096 \\
\hline I comprehend the scope of practice. & 13.42 & 0.0094 \\
\hline $\begin{array}{l}\text { I refer friends and family to other sources that are outside my scope of } \\
\text { practice. }\end{array}$ & 1.19 & 0.7556 \\
\hline I abide by the Dietetics Code of Ethics. & 2.07 & 0.7224 \\
\hline $\begin{array}{l}\text { I use nutrition information to diagnose health conditions in family } \\
\text { members and individuals. }\end{array}$ & 1.96 & 0.1611 \\
\hline Major & & \\
\hline
\end{tabular}


Table 91 (Part A): CREDIBILITY AND RELIABILITY BEHAVIORS: Individuals and family members believe my information is credible based on my years in school.

\begin{tabular}{|c|c|c|c|c|c|c|c|}
\hline \multicolumn{8}{|c|}{$\begin{array}{l}\text { CREDIBILITY AND RELIABILITY BEHAVIORS: Individuals and family members believe my } \\
\text { information is credible based on my years in school. }\end{array}$} \\
\hline \multirow{5}{*}{\begin{tabular}{|l} 
Parameter \\
I only deliver \\
information allowed \\
at the student level by \\
the CDR, as specified \\
in the Dietetic Scope \\
of Practice.
\end{tabular}} & \multirow{2}{*}{$\begin{array}{l}\text { Response } \\
\text { Disagree }\end{array}$} & \multirow{2}{*}{\begin{tabular}{|r|} 
Estimate \\
-0.4927
\end{tabular}} & \multirow{2}{*}{$\begin{array}{l}\text { Standard } \\
\text { Error } \\
2.0520\end{array}$} & \multicolumn{2}{|c|}{$\begin{array}{l}\text { Wald 95\% } \\
\text { Confidence Limits }\end{array}$} & \multirow{2}{*}{\begin{tabular}{|r|}
$\begin{array}{l}\text { Wald } \\
\text { Chi-Square }\end{array}$ \\
0.06
\end{tabular}} & \multirow{2}{*}{$\begin{array}{l}\text { Pr > } \\
\text { ChiSq } \\
0.8102\end{array}$} \\
\hline & & & & -4.5145 & 3.5291 & & \\
\hline & Neutral & 0.0010 & 1.0094 & -1.9774 & 1.9794 & 0.00 & 0.9992 \\
\hline & Agree & 0.2074 & 1.0861 & -1.9213 & 2.3362 & 0.04 & 0.8486 \\
\hline & $\begin{array}{l}\text { Strongly } \\
\text { Agree }\end{array}$ & 0.0000 & 0.0000 & 0.0000 & 0.0000 & & \\
\hline \multirow{5}{*}{$\begin{array}{l}\text { I comprehend the } \\
\text { scope of practice. }\end{array}$} & $\begin{array}{l}\text { Strongly } \\
\text { Disagree }\end{array}$ & -2.8370 & 2.3117 & -7.3680 & 1.6939 & 1.51 & 0.2197 \\
\hline & Disagree & -2.7877 & 1.6374 & -5.9969 & 0.4215 & 2.90 & 0.0887 \\
\hline & Neutral & -2.4900 & 1.1622 & -4.7678 & -0.2121 & 4.59 & 0.0322 \\
\hline & Agree & -1.7971 & 1.0738 & -3.9018 & 0.3075 & 2.80 & 0.0942 \\
\hline & $\begin{array}{l}\text { Strongly } \\
\text { Agree }\end{array}$ & 0.0000 & 0.0000 & 0.0000 & 0.0000 & & \\
\hline \multirow{5}{*}{$\begin{array}{l}\text { I refer friends and } \\
\text { family to other } \\
\text { sources that are } \\
\text { outside my scope of } \\
\text { practice. }\end{array}$} & $\begin{array}{l}\text { Strongly } \\
\text { Disagree }\end{array}$ & -2.8226 & 2.3628 & -7.4536 & 1.8085 & 1.43 & 0.2323 \\
\hline & Disagree & -0.9674 & 1.8798 & -4.6517 & 2.7169 & 0.26 & 0.6068 \\
\hline & Neutral & -2.1778 & 0.7991 & -3.7441 & -0.6116 & 7.43 & 0.0064 \\
\hline & Agree & -1.9760 & 0.7129 & -3.3733 & -0.5787 & 7.68 & 0.0056 \\
\hline & $\begin{array}{l}\text { Strongly } \\
\text { Agree }\end{array}$ & 0.0000 & 0.0000 & 0.0000 & 0.0000 & & \\
\hline \multirow{4}{*}{$\begin{array}{l}\text { I abide by the } \\
\text { Dietetics Code of } \\
\text { Ethics. }\end{array}$} & Disagree & 1.9926 & 2.2343 & -2.3865 & 6.3718 & 0.80 & 0.3725 \\
\hline & Neutral & -1.1762 & 0.9492 & -3.0365 & 0.6841 & 1.54 & 0.2153 \\
\hline & Agree & 0.2092 & 0.8084 & -1.3752 & 1.7937 & 0.07 & 0.7958 \\
\hline & $\begin{array}{l}\text { Strongly } \\
\text { Agree }\end{array}$ & 0.0000 & 0.0000 & 0.0000 & 0.0000 & & \\
\hline \multirow{5}{*}{$\begin{array}{l}\text { I use nutrition } \\
\text { information to } \\
\text { diagnose health } \\
\text { conditions in family } \\
\text { members and } \\
\text { individuals. }\end{array}$} & $\begin{array}{l}\text { Strongly } \\
\text { Disagree }\end{array}$ & 1.0654 & 1.1599 & -1.2080 & 3.3387 & 0.84 & 0.3584 \\
\hline & Disagree & 0.5043 & 0.9260 & -1.3106 & 2.3193 & 0.30 & 0.5860 \\
\hline & Neutral & 1.6743 & 0.9593 & -0.2059 & 3.5546 & 3.05 & 0.0809 \\
\hline & Agree & 1.0909 & 1.0023 & -0.8736 & 3.0554 & 1.18 & 0.2764 \\
\hline & $\begin{array}{l}\text { Strongly } \\
\text { Agree }\end{array}$ & 0.0000 & 0.0000 & 0.0000 & 0.0000 & & \\
\hline \multirow{2}{*}{ Major } & Dietetics & 0.3482 & 0.6044 & -0.8365 & 1.5328 & 0.33 & 0.5646 \\
\hline & Human Ntr & 0.0000 & 0.0000 & 0.0000 & 0.0000 & & \\
\hline
\end{tabular}


Table 91 (Part B): CREDIBILITY AND RELIABILITY BEHAVIORS: Individuals and family members believe my information is credible based on my years in school.

\begin{tabular}{|l|r|r|}
\hline \multicolumn{3}{|c|}{$\begin{array}{l}\text { CREDIBILITY AND RELIABILITY BEHAVIORS: Individuals and family members believe my } \\
\text { information is credible based on my years in school. }\end{array}$} \\
\hline Source & Chi-Square & Pr > ChiSq \\
\hline $\begin{array}{l}\text { I only deliver information allowed at the student level by the CDR, as } \\
\text { specified in the Dietetic Scope of Practice. }\end{array}$ & 0.18 & 0.9814 \\
\hline I comprehend the scope of practice. & 5.97 & 0.2016 \\
\hline $\begin{array}{l}\text { I refer friends and family to other sources that are outside my scope of } \\
\text { practice. }\end{array}$ & 10.64 & 0.0309 \\
\hline I abide by the Dietetics Code of Ethics. & 5.92 & 0.1156 \\
\hline $\begin{array}{l}\text { I use nutrition information to diagnose health conditions in family } \\
\text { members and individuals. }\end{array}$ & 4.66 & 0.3235 \\
\hline Major & 0.33 & 0.5639 \\
\hline
\end{tabular}


Table 92 (Part A): CREDIBILITY AND RELIABILITY BEHAVIORS: My height and weight support my credibility with family and friends.

\begin{tabular}{|c|c|c|c|c|c|c|c|}
\hline \multicolumn{8}{|c|}{$\begin{array}{l}\text { CREDIBILITY AND RELIABILITY BEHAVIORS: My height and weight support my credibility with } \\
\text { family and friends. }\end{array}$} \\
\hline \multirow{5}{*}{$\begin{array}{l}\text { Parameter } \\
\text { I only deliver } \\
\text { information } \\
\text { allowed at the } \\
\text { student level by } \\
\text { the CDR, as } \\
\text { specified in the } \\
\text { Dietetic Scope of } \\
\text { Practice. }\end{array}$} & \multirow{2}{*}{\begin{tabular}{|l} 
Response \\
Disagree
\end{tabular}} & \multirow{2}{*}{$\begin{array}{r}\text { Estimate } \\
1.5637\end{array}$} & \multirow{2}{*}{\begin{tabular}{|l|}
$\begin{array}{l}\text { Standard } \\
\text { Error }\end{array}$ \\
1.9164
\end{tabular}} & \multicolumn{2}{|c|}{$\begin{array}{l}\text { Wald } 95 \% \\
\text { Confidence Limits }\end{array}$} & \multirow{2}{*}{\begin{tabular}{|r}
$\begin{array}{l}\text { Wald } \\
\text { Chi-Square }\end{array}$ \\
0.67
\end{tabular}} & \multirow{2}{*}{$\begin{array}{l}\mathrm{Pr}> \\
\text { ChiSq } \\
0.4145\end{array}$} \\
\hline & & & & -2.1924 & 5.3198 & & \\
\hline & Neutral & 2.4649 & 1.0067 & 0.4918 & 4.4380 & 5.99 & 0.0143 \\
\hline & Agree & 2.0531 & 1.0613 & -0.0270 & 4.1332 & 3.74 & 0.0531 \\
\hline & $\begin{array}{l}\text { Strongly } \\
\text { Agree }\end{array}$ & 0.0000 & 0.0000 & 0.0000 & 0.0000 & & \\
\hline \multirow{5}{*}{$\begin{array}{l}\text { I comprehend the } \\
\text { scope of practice. }\end{array}$} & & 20.8454 & 93658.60 & -183547 & 183588.3 & 0.00 & 0.9998 \\
\hline & Disagree & -3.2744 & 1.4796 & -6.1743 & -0.3744 & 4.90 & 0.0269 \\
\hline & Neutral & -2.9714 & 1.1119 & -5.1507 & -0.7921 & 7.14 & 0.0075 \\
\hline & Agree & -3.2736 & 1.0312 & -5.2947 & -1.2525 & 10.08 & 0.0015 \\
\hline & $\begin{array}{l}\text { Strongly } \\
\text { Agree }\end{array}$ & 0.0000 & 0.0000 & 0.0000 & 0.0000 & & \\
\hline \multirow{5}{*}{$\begin{array}{l}\text { I refer friends and } \\
\text { family to other } \\
\text { sources that are } \\
\text { outside my scope } \\
\text { of practice. }\end{array}$} & $\begin{array}{l}\text { Strongly } \\
\text { Disagree }\end{array}$ & -1.7605 & 1.9981 & -5.6768 & 2.1557 & 0.78 & 0.3783 \\
\hline & Disagree & -1.9852 & 1.6629 & -5.2444 & 2739 & 1.43 & 0.2325 \\
\hline & Neutral & -2.8602 & 0.8258 & -4.4786 & -1.2417 & 12.00 & 0.0005 \\
\hline & Agree & -2.2080 & 0.7302 & -3.6392 & -0.7769 & 9.14 & 0.0025 \\
\hline & $\begin{array}{l}\text { Strongly } \\
\text { Agree }\end{array}$ & 0.0000 & 0.0000 & 0.0000 & 0.0000 & & \\
\hline \multirow{4}{*}{$\begin{array}{l}\text { I abide by the } \\
\text { Dietetics Code of } \\
\text { Ethics. }\end{array}$} & Disagree & -0.7178 & 2.0133 & -4.6637 & 3.2282 & 0.13 & 0.7214 \\
\hline & Neutral & 0.4687 & 0.8794 & -1.2549 & 2.1923 & 0.28 & 0.5940 \\
\hline & Agree & 1.2437 & 0.7608 & -0.2475 & 2.7349 & 2.67 & 0.1021 \\
\hline & $\begin{array}{l}\text { Strongly } \\
\text { Agree }\end{array}$ & 0.0000 & 0.0000 & 0.0000 & 0.0000 & & \\
\hline \multirow{5}{*}{$\begin{array}{l}\text { I use nutrition } \\
\text { information to } \\
\text { diagnose health } \\
\text { conditions in } \\
\text { family members } \\
\text { and individuals. }\end{array}$} & $\begin{array}{l}\text { Strongly } \\
\text { Disagree }\end{array}$ & -1.2901 & 1.1269 & -3.4987 & 0.9185 & 1.31 & 0.2523 \\
\hline & Disagree & -0.1210 & 0.9241 & -1.9322 & 1.6902 & 0.02 & 0.8958 \\
\hline & Neutral & 0.0338 & 0.9400 & -1.8086 & 1.8761 & 0.00 & 0.9713 \\
\hline & Agree & -0.2900 & 0.9817 & -2.2141 & 1.6340 & 0.09 & 0.7677 \\
\hline & $\begin{array}{l}\text { Strongly } \\
\text { Agree }\end{array}$ & 0.0000 & 0.0000 & 0.0000 & 0.0000 & & \\
\hline \multirow{2}{*}{ Major } & Dietetics & 1.5293 & 0.5684 & 0.4153 & 2.6434 & 7.24 & 0.0071 \\
\hline & Human Ntr & 0.0000 & 0.0000 & 0.0000 & 0.0000 & & \\
\hline
\end{tabular}


Table 92 (Part B): CREDIBILITY AND RELIABILITY BEHAVIORS: My height and weight support my credibility with family and friends.

\begin{tabular}{|c|c|c|}
\hline $\begin{array}{l}\text { CREDIBILITY AND RELIABILITY BEHAVIORS: My height and weight } s \\
\text { family and friends. }\end{array}$ & port my cr & bility with \\
\hline Source & Chi-Square & Pr $>$ ChiSq \\
\hline $\begin{array}{l}\text { I only deliver information allowed at the student level by the CDR, as } \\
\text { specified in the Dietetic Scope of Practice. }\end{array}$ & 5.92 & 0.1155 \\
\hline I comprehend the scope of practice. & 14.27 & 0.0065 \\
\hline $\begin{array}{l}\text { I refer friends and family to other sources that are outside my scope of } \\
\text { practice. }\end{array}$ & 14.55 & 0.0057 \\
\hline I abide by the Dietetics Code of Ethics. & 3.84 & 0.2794 \\
\hline $\begin{array}{l}\text { I use nutrition information to diagnose health conditions in family } \\
\text { members and individuals. }\end{array}$ & 2.10 & 0.7169 \\
\hline Major & 7.57 & 0.0059 \\
\hline
\end{tabular}


Table 93 (Part A): CREDIBILITY AND RELIABILITY BEHAVIORS: My health behaviors (eating and exercise) support my credibility with family and individuals.

\begin{tabular}{|c|c|c|c|c|c|c|c|}
\hline \multicolumn{8}{|c|}{$\begin{array}{l}\text { CREDIBILITY AND RELIABILITY BEHAVIORS: My health behaviors (eating and exercise) support } \\
\text { my credibility with family and individuals. }\end{array}$} \\
\hline \multirow{5}{*}{$\begin{array}{l}\text { Parameter } \\
\text { I only deliver } \\
\text { information allowed } \\
\text { at the student level } \\
\text { by the CDR, as } \\
\text { specified in the } \\
\text { Dietetic Scope of } \\
\text { Practice. }\end{array}$} & \multirow{2}{*}{$\begin{array}{l}\text { Response } \\
\text { Disagree }\end{array}$} & \multirow{2}{*}{$\begin{array}{r}\text { Estimate } \\
0.3390\end{array}$} & \multirow{2}{*}{\begin{tabular}{|r|}
$\begin{array}{l}\text { Standard } \\
\text { Error }\end{array}$ \\
1.9403
\end{tabular}} & \multicolumn{2}{|c|}{$\begin{array}{l}\text { Wald 95\% } \\
\text { Confidence Limits }\end{array}$} & \multirow{2}{*}{$\begin{array}{l}\text { Wald } \\
\text { Chi-Square } \\
0.03\end{array}$} & \multirow{2}{*}{\begin{tabular}{|l|} 
Pr $>$ \\
ChiSq
\end{tabular}} \\
\hline & & & & -3.4639 & 4.1419 & & \\
\hline & Neutral & 0.5783 & 0.8831 & -1.1526 & 2.3093 & 0.43 & 0.5126 \\
\hline & Agree & 0.8855 & 0.9162 & -0.9102 & 2.6813 & 0.93 & 0.3338 \\
\hline & $\begin{array}{l}\text { Strongly } \\
\text { Agree }\end{array}$ & 0.0000 & 0.0000 & 0.0000 & 0.0000 & & \\
\hline \multirow{5}{*}{$\begin{array}{l}\text { I comprehend the } \\
\text { scope of practice. }\end{array}$} & $\begin{array}{l}\text { Strongly } \\
\text { Disagree }\end{array}$ & -2.2470 & 1.9079 & -5.9865 & 1.4925 & 1.39 & 0.2389 \\
\hline & Disagree & -0.9934 & 1.3717 & -3.6820 & 1.6951 & 0.52 & 0.4689 \\
\hline & Neutral & -0.3070 & 0.9020 & -2.0748 & 1.4609 & 0.12 & 0.7336 \\
\hline & Agree & -1.1647 & 0.8249 & -2.7814 & 0.4520 & 1.99 & 0.1579 \\
\hline & $\begin{array}{l}\text { Strongly } \\
\text { Agree }\end{array}$ & 0.0000 & 0.0000 & 0.0000 & 0.0000 & & \\
\hline \multirow{5}{*}{$\begin{array}{l}\text { I refer friends and } \\
\text { family to other } \\
\text { sources that are } \\
\text { outside my scope of } \\
\text { practice. }\end{array}$} & $\begin{array}{l}\text { Strongly } \\
\text { Disagree }\end{array}$ & -0.4316 & 1.9161 & -4.1871 & 3.3240 & 0.05 & 0.8218 \\
\hline & Disagree & -3.3634 & 1.6366 & -6.5711 & -0.1558 & 4.22 & 0.0399 \\
\hline & Neutral & -1.3708 & 0.7121 & -2.7665 & 0.0248 & 3.71 & 0.0542 \\
\hline & Agree & -0.8311 & 0.6248 & -2.0556 & 0.3934 & 1.77 & 0.1834 \\
\hline & $\begin{array}{l}\text { Strongly } \\
\text { Agree }\end{array}$ & 0.0000 & 0.0000 & 0.0000 & 0.0000 & & \\
\hline \multirow{4}{*}{$\begin{array}{l}\text { I abide by the } \\
\text { Dietetics Code of } \\
\text { Ethics. }\end{array}$} & Disagree & -2.1251 & 2.1507 & -6.3405 & 2.0902 & 0.98 & 0.3231 \\
\hline & Neutral & -1.9685 & 0.8234 & -3.5822 & -0.3547 & 5.72 & 0.0168 \\
\hline & Agree & -0.9348 & 0.6560 & -2.2206 & 0.3509 & 2.03 & 0.1541 \\
\hline & $\begin{array}{l}\text { Strongly } \\
\text { Agree }\end{array}$ & 0.0000 & 0.0000 & 0.0000 & 0.0000 & & \\
\hline \multirow{5}{*}{$\begin{array}{l}\text { I use nutrition } \\
\text { information to } \\
\text { diagnose health } \\
\text { conditions in family } \\
\text { members and } \\
\text { individuals. }\end{array}$} & $\begin{array}{l}\text { Strongly } \\
\text { Disagree }\end{array}$ & -0.3544 & 1.0476 & -2.4077 & 1.6988 & 0.11 & 0.7351 \\
\hline & Disagree & 0.3241 & 0.8900 & -1.4203 & 2.0685 & 0.13 & 0.7158 \\
\hline & Neutral & 0.4998 & 0.8679 & -1.2013 & 2.2009 & 0.33 & 0.5647 \\
\hline & Agree & 0.5130 & 0.9462 & -1.3415 & 2.3675 & 0.29 & 0.5877 \\
\hline & $\begin{array}{l}\text { Strongly } \\
\text { Agree }\end{array}$ & 0.0000 & 0.0000 & 0.0000 & 0.0000 & & \\
\hline \multirow{2}{*}{ Major } & Dietetics & 1.3472 & 0.5558 & 0.2579 & 2.4366 & 5.88 & 0.0154 \\
\hline & Human Ntr & 0.0000 & 0.0000 & 0.0000 & 0.0000 & & \\
\hline
\end{tabular}


Table 93 (Part B): CREDIBILITY AND RELIABILITY BEHAVIORS: My health behaviors (eating and exercise) support my credibility with family and individuals.

\begin{tabular}{|c|c|c|}
\hline Source & Chi-Square & $\operatorname{Pr}>$ ChiSq \\
\hline $\begin{array}{l}\text { I only deliver information allowed at the student level by the CDR, as } \\
\text { specified in the Dietetic Scope of Practice. }\end{array}$ & 0.98 & 0.8056 \\
\hline I comprehend the scope of practice. & 4.57 & 0.3344 \\
\hline $\begin{array}{l}\text { I refer friends and family to other sources that are outside my scope of } \\
\text { practice. }\end{array}$ & 6.70 & 0.1526 \\
\hline I abide by the Dietetics Code of Ethics. & 6.34 & 0.0961 \\
\hline $\begin{array}{l}\text { I use nutrition information to diagnose health conditions in family } \\
\text { members and individuals. }\end{array}$ & 1.27 & 0.8658 \\
\hline Major & 6.01 & 0.0142 \\
\hline
\end{tabular}


Table 94 (Part A): CREDIBILITY AND RELIABILITY BEHAVIORS: I incorporate low fat, high fiber foods into my diet on a regular basis.

\begin{tabular}{|c|c|c|c|c|c|c|c|}
\hline CREDIBILITY AN & RELIABILI' & $\begin{array}{r}\text { 3EHAVIOF } \\
\text { on a }\end{array}$ & $\begin{array}{l}\text { RS: I incorl } \\
\text { regular ba }\end{array}$ & ate low & igh fib & foods int & $y$ diet \\
\hline Parameter & Response & Estimate & $\begin{array}{l}\text { Standard } \\
\text { Error }\end{array}$ & $\begin{array}{l}\text { Wald 95\% } \\
\text { Confidenc }\end{array}$ & Limits & $\begin{array}{l}\text { Wald } \\
\text { Chi-Square }\end{array}$ & $\begin{array}{l}\text { Pr }> \\
\text { ChiSq }\end{array}$ \\
\hline I only deliver & Disagree & -2.1029 & 1.9352 & -5.8957 & 1.6899 & 1.18 & 0.2772 \\
\hline $\begin{array}{l}\text { information } \\
\text { allowed at the }\end{array}$ & Neutral & 1.1866 & 0.9392 & -0.6542 & 3.0274 & 1.60 & 0.2064 \\
\hline student level by the & Agree & 1.6494 & 0.9914 & -0.2937 & 3.5925 & 2.77 & 0.0962 \\
\hline $\begin{array}{l}\text { the Dietetic Scope } \\
\text { of Practice. }\end{array}$ & $\begin{array}{l}\text { Strongly } \\
\text { Agree }\end{array}$ & 0.0000 & 0.0000 & 0.0000 & 0.0000 & & \\
\hline & $\begin{array}{l}\text { Strongly } \\
\text { Disagree }\end{array}$ & 22.5058 & 97526.75 & -191126 & 191171.4 & 0.00 & 0.9998 \\
\hline & Disagree & -0.4141 & 1.4503 & -3.2567 & 2.4285 & 0.08 & 0.7753 \\
\hline $\begin{array}{l}1 \text { comprenena the } \\
\text { scope of practice. }\end{array}$ & Neutral & -0.4914 & 0.9791 & -2.4104 & 1.4276 & 0.25 & 0.6157 \\
\hline & Agree & -0.9442 & 0.9023 & -2.7128 & 0.8244 & 1.09 & 0.2954 \\
\hline & $\begin{array}{l}\text { Strongly } \\
\text { Agree }\end{array}$ & 0.0000 & 0.0000 & 0.0000 & 0.0000 & & \\
\hline & $\begin{array}{l}\text { Strongly } \\
\text { Disagree }\end{array}$ & -2.9327 & 2.1130 & -7.0741 & 1.2088 & 1.93 & 0.1652 \\
\hline I refer friends and & Disagree & -6.1365 & 1.8995 & -9.8595 & -2.4135 & 10.44 & 0.0012 \\
\hline sources that are & Neutral & -1.6033 & 0.7498 & -3.0728 & -0.1338 & 4.57 & 0.0325 \\
\hline $\begin{array}{l}\text { outside my scope or } \\
\text { practice. }\end{array}$ & Agree & -1.6747 & 0.6629 & -2.9740 & -0.3755 & 6.38 & 0.0115 \\
\hline & $\begin{array}{l}\text { Strongly } \\
\text { Agree }\end{array}$ & 0.0000 & 0.0000 & 0.0000 & 0.0000 & & \\
\hline & Disagree & 3.7393 & 2.1349 & -0.4450 & 7.9236 & 3.07 & 0.0799 \\
\hline I abide by the & Neutral & -0.9220 & 0.8103 & -2.5101 & 0.6661 & 1.29 & 0.2552 \\
\hline Dietetics Code of & Agree & -0.6065 & 0.6664 & -1.9126 & 0.6997 & 0.83 & 0.3628 \\
\hline & $\begin{array}{l}\text { Strongly } \\
\text { Agree }\end{array}$ & 0.0000 & 0.0000 & 0.0000 & 0.0000 & & \\
\hline I use nutrition & $\begin{array}{l}\text { Strongly } \\
\text { Disagree }\end{array}$ & 0.1759 & 1.1373 & -2.0532 & 2.4049 & 0.02 & 0.8771 \\
\hline information to & Disagree & 0.0661 & 0.8981 & -1.6941 & 1.8262 & 0.01 & 0.9413 \\
\hline $\begin{array}{l}\text { diagnose health } \\
\text { conditions in family }\end{array}$ & Neutral & -0.2357 & 0.8887 & -1.9775 & 1.5061 & 0.07 & 0.7908 \\
\hline members and & Agree & -0.1818 & 0.9342 & -2.0129 & 1.6493 & 0.04 & 0.8457 \\
\hline & $\begin{array}{l}\text { Strongly } \\
\text { Agree }\end{array}$ & 0.0000 & 0.0000 & 0.0000 & 0.0000 & & \\
\hline Main & Dietetics & 1.1953 & 0.5819 & 0.0548 & 2.3358 & 4.22 & 0.0400 \\
\hline & Human Ntr & 0.0000 & 0.0000 & 0.0000 & 0.0000 & & \\
\hline
\end{tabular}


Table 94 (Part B): CREDIBILITY AND RELIABILITY BEHAVIORS: I incorporate low fat, high fiber foods into my diet on a regular basis.

\begin{tabular}{|c|c|c|}
\hline \multicolumn{3}{|c|}{$\begin{array}{l}\text { CREDIBILITY AND RELIABILITY BEHAVIORS: I incorporate low fat, high fiber foods into my diet } \\
\text { on a regular basis. }\end{array}$} \\
\hline Source & Chi-Square & $\operatorname{Pr}>$ ChiSq \\
\hline $\begin{array}{l}\text { I only deliver information allowed at the student level by the CDR, as } \\
\text { specified in the Dietetic Scope of Practice. }\end{array}$ & 5.95 & 0.1139 \\
\hline I comprehend the scope of practice. & 2.88 & 0.5785 \\
\hline $\begin{array}{l}\text { I refer friends and family to other sources that are outside my scope of } \\
\text { practice. }\end{array}$ & 15.33 & 0.0041 \\
\hline I abide by the Dietetics Code of Ethics. & 5.60 & 0.1328 \\
\hline $\begin{array}{l}\text { I use nutrition information to diagnose health conditions in family } \\
\text { members and individuals. }\end{array}$ & 0.35 & 0.9866 \\
\hline Major & 4.32 & 0.0377 \\
\hline
\end{tabular}




\section{References}

American Dietetic Association/Commission on Dietetic Registration. (2009). "Code of Ethics for the Profession of Dietetics and Process for Consideration of Ethics Issues." Journal of the American Dietetic Association, 109(8), 1461-1467. doi: 10.1016/j.jada.2009.06.002

Bidlack, W.R. (1996). "Interrelationships of food, nutrition, diet and health: the National Association of State Universities and Land Grant Colleges White Paper." Journal of the American College of Nutrition, 15(5), 422-433.

Blackburn, Mary L., Townsend, Marilyn S., Kaiser, Lucia L., Martin, Anna C., West, Estella A., Turner, Barbara, Joy, Amy B. (2006). "Food behavior checklist effectively evaluates nutrition education." California Agriculture 60(1). Retrieved from http://CaliforniaAgriculture.ucop.edu

Branen, Laurel, Fletcher, Janice. (1999). "Comparison of College Students' Current Eating Habits and Recollections of Their Childhood Food Practices." Journal of Nutrition Education, 31(6), 304-310. doi: 10.1016/S0022-3182(99)70483-8

Cousineau, Tara M., PhD, Goldstein, Marion, BA, Franko, Debra L., PhD. (2004). "A Collaborative Approach to Nutrition Education for College Students." Journal of American College Health, 53(2).

Crombie, Aaron P., Ilich, Jasminka Z., Dutton, Gareth R., Panton, Lynn B., Abood, Doris A. (2009). "The freshman weight gain phenomenon revisited." Nutrition Reviews, 67(2), 83-94. doi:10.1111/j.1753-4887.2008.00143.x

Davy, Sarah R., Benes, Beverly A., PhD, RD, Driskell, Judy A., PhD, RD. (2006). “Sex Differences in Dieting Trends, Eating Habits, and Nutrition Beliefs of a Group of Midwestern College Students." Journal of the American Dietetic Association 106(10). doi: 10.1016/j.jada.2006.07.017

Driskell, Judy A., PhD, RD, Kim, Young-Nam, MS, Goebel, Kelly J. (2005). "Few Differences Found in the Typical Eating and Physical Activity Habits of Lower-Level and Upper-Level University Students." Journal of the American Dietetic Association 105(5). doi: 10.1016/j.jada.2005.02.004

Driskell, Judy A., PhD, RD, Schake, Marian C., RD, Detter, Hillary A., RD. (2008). "Using Nutrition Labeling as a Potential Tool for Changing Eating Habits of University Dining Hall Patrons." Journal of the American Dietetic Association, 108(12), 2071-2076. doi: 10.1016/j.jada.2008.09.009

Ferrera, Cynthia M. (2009). "The College Experience: Physical Activity, Nutrition, and Implications for Intervention and Future Research." Journal of Exercise 
Physiologyonline (JEPonline) 12(1).

Graham, Melody A. and Jones, Amy L. (2002). "Freshman 15: Valid Theory or Harmful Myth?" Journal of American College Health, 50(4), 171-173. doi: $10.1080 / 07448480209596023$

Greaney, Mary L., PhD, Less, Faith D., MS, White, Adrienne A., PhD, RD, Dayton, Sarah F., MS, Reibe, Deborah, PhD, Blissmer, Bryan, PhD, Shoff, Suzanne, PhD, Walsh, Jennifer R., MS, RD, Greene, Geoffrey W., PhD, RD, LDN. (2009). "College Students' Barriers and Enablers for Healthful Weight Management: A Qualitative Study." Journal of Nutrition Education and Behavior, 41(4) 281-286. doi: 10.1016/j.jneb.2008.04.354

Holm-Denoma, Jill M., Joiner Jr., Thomas E., Vohs, Kathleen D., Heatherton, Todd F. “The 'Freshman Fifteen' (the 'Freshman Five' Actually): Predictors and Possible Explanations. (2008). Health Psychology, 27(1), S3-S9. doi: 10.1037/0278-6133.27.1.S3

James, Katie J. (2010). Assessing Dietary Intake, Eating and Exercise Attitudes and Fitness Levels in College-Aged Students (Master's thesis). University of Nebraska - Lincoln.

Kiecolt-Glaser, Janice K., PhD. (2010). "Stress, Food, and Inflammation: Psychoneuroimmunology and Nutrition at the Cutting Edge." Psychosomatic Medicine. doi: 0033-3174/10/7205-0001

Kolodinsky, Jane, PhD, Green, Jennifer, PhD, Michahelles, Marina, MS, Harvey-Berino, Jean R., PhD. (2008). "The Use of Nutritional Labels by College Students in a Food-Court Setting." Journal of American College Health, 57(3), 297-301.

Levitsky, DA, Halbmaier, CA, Mrdjenovic, G. (2004). "The freshman weight gain: a model for the study of the epidemic of obesity." International Journal of Obesity Related Metabolic Disorders, 28(11), 1435-1442.

Levitsky, David A., Youn, Trisha. (2004). "The More Foods Young Adults Are Served, the More They Overeat." The Journal of Nutrition, 134(10), 2546-2549.

Liu, Chunhong, et al. (2007). "Perceived stress, depression and food consumption frequency in the college students of China seven cities." Physiology \& Behavior, 92(4), 748-754.

Lowry, Richard, MD, MS, Galuska, Deborah A., PhD, Fulton, Janet E., PhD, Wechsler, Howell, EdD, MPH, Kann, Laura, PhD, Collins, Janet L., PhD. (2000). "Physical Activity, Food Choice, and Weight Management Goals and Practices Among U.S. College Students." American Journal of Preventative Medicine 18(1), 18-27. 
Maillet, Julie O'Sullivan, PhD, RD, FADA, Skates, Janet, MS, RD, FADA, Pritchett, Ellen, RD. (2005). "American Dietetic Association: Scope of Dietetics Practice Framework." Journal of the American Dietetic Association 105(4). doi: 10.1016/j.jada.2005.02.001

Malinauskas, Brenda M., Raedeke, Thomas D., Aeby, Victor G., Smith, Jean L., Dallas, Matthew B. (2006). "Dieting practices, weight perceptions, and body composition: A comparison of normal weight, overweight, and obese college females." Nutrition Journal, 5(11). doi: 10.1186/1475-2891-5-11

Mihalopoulos, Nicole L. , Auinger, Peggy and Klein, Jonathan D. (2008). "The Freshman 15: Is it Real?" Journal of American College Health, 56(5), 531-534. doi: 10.3200/JACH.56.5.531-534

Mikolajczyk, Rafael T., Ansari, Walid El, Maxwell, Annette E. (2009) Food consumption frequency and perceived stress and depressive symptoms among students in three European countries. Nutrition Journal, 8(31). doi: 10.1186/1475-2891-8-31

Nayga, Rodolfo M., jr. (2000). "Nutrition Knowledge, Gender, and Food Label Use." The Journal of Consumer Affairs 34(1), 97-112. doi: 0022-0078/0002- I

Nelson, Melissa C., PhD, RD, Kocos, Rebecca, MPH, Lytle, Leslie A., PhD, RD, Perry, Cheryl L., PhD. (2009). "Understanding the Perceived Determinants of Weight-related Behaviors in Late Adolescence: A Qualitative Analysis among College Youth." Journal of Nutrition Education, 41(4), 287-292. doi: 10.1016/j.jneb.2008.05.005

"Nutrition Labeling." (2010). American Heart Association. Retrieved from http://www.heart.org/HEARTORG/GettingHealthy/NutritionCenter/Nutriti on-Center_UCM_001188_SubHomePage.jsp

Parmenter, K., Wardle, J. (1999). "Development of a general nutrition knowledge questionnaire for adults." European Journal of Clinical Nutrition, 53, 298-308. Stockton Press.

Peterson, Sharon, PhD, RD, Duncan, Diana Poovey, MS, RD, Null, Dawn Boyd, MS, RD, Roth, Sara Long, PhD, RD, Gill, Lynn, MS, RD. (2010). "Positive Changes in Perceptions and Selections of Healthful Foods by College Students After a Short-Term Point-of-Selection Intervention at a Dining Hall." Journal of American College Health, 58(5), 425-431.

Pritchard, Mary E., PhD, Wilson, Gregory S., PED, Yamnitz, Ben, BS. (2007). "What Predicts Adjustment Among College Students? A Longitudinal Panel Study." Journal of American College Health, 56(1), 15-21. 
Pullman, AW, Masters, RC, Zalot, LC, Carde, LE, Saraiva, MM, Dam, YY, Randall Simpson, JA, Duncan, AM. (2009). "Effect of the transition from high school to university on anthropometric and lifestyle variables in males." Applied Physiology, Nutrition, and Metabolism, 34(2), 162-171.

Robles, Delma S. (2010). “Construct Validation of an Eating Behavior Checklist.” The Assessment Handbook, Vol. 3. ISSN 2094-1412, PEMEA.

Strong, Kathryn A., MS, Parks, Serena L., Anderson, Eileen, EdD, Winett, Richard, PhD, Davy, Brenda M., PhD, RD. (2008). "Weight Gain Prevention: Identifying Theory-Based Targets for Health Behavior Change in Young Adults." Journal of the American Dietetic Association, 108(10), 1708-1715.e3. doi: 10.1016/j.jada.2008.07.007

"The Regions of the United States." (2008). U.S. Diplomatic Mission to Germany. Retrieved from http://usa.usembassy.de/travel-regions.htm

Torres, Susan J., M.Nutr.Diet, Nowson, Carol A, PhD. (2007). "Relationship between stress, eating behavior, and obesity." The Journal of Nutrition, 23, 887-894. doi: 10.1016/j.nut.2007.08.008

USDA Center for Nutrition Policy and Promotion. (2000). "Serving Sizes in the Food Guide Pyramid and on the Nutrition Facts Label: What's Different and Why?" Nutrition Insights, 22. Retrieved from http://www.usda.gov/cnpp

van Dillen, Sonja ME, Heddink, Gerrit Jan, Koelen, Maria A., de Graaf, Cees, van Woerkum, Cees MJ. (2007) "Exploration of possible correlates of nutrition awareness and the relationship with nutrition-related behaviours: results of a consumer study." Public Health Nutrition 11(5), 478-485. doi: 10.1017/S1368980007000754

Vella-Zarb, RA., Elgar, FJ. (2009). "The 'freshman 5': a meta-analysis of weight gain in the freshman year of college." Journal of American College Health, 58(2), 161-166.

Wainsink, Brian, Painter, James E., North, Jill. (2005). “Bottomless Bowls: Why Visual Cues of Portion Size May Influence Intake.” Obesity Research, 13(1), 93-100.

Wardlaw, Gordon M., Hampl, Jeffry S., and DiSilvestro, Robert. A. (2004), "Perspectives: Vegetarian Diets." Perspectives in Nutrition, 6/e. McGraw Hill. New York, NY.

Wardle, Jane, Steptoe, Andrew, Oliver, Georgina, Lipsey, Zara. (1999). "Stress, dietary restrain, and food intake." Journal of Psychosomatic Research, 48(2). 195-202. 
Weingarten, Harvey P., Elston, Dawn. (1991). "Food cravings in a college population." Appetite, 17(3), 167-175. doi: 10.1016/0195-6663(91)90019-0

Werch, Chudley E. (Chad), PhD, Bian, Hui, PhD, Moore, Michele J., PhD, Ames, Steve, PhD, DiClemente, Carlo C., PhD, Weiler, Robert M., PhD. (2007). "Brief Multiple Behavior Interventions in a College Student Health Care Clinic." Journal of Adolescent Health 41(6).

Winterfeldt, Esther A., Bogle, Margaret L., Ebro, Lea L. (2011). Dietetics Practice and Future Trends, 3/e. Jones and Bartlett Publishers, LLC. London, United Kingdom. 
Vita:

$$
\text { Jennifer Kristin Boyee }
$$

Date and Place of Birth:

November 15, 1985

Dayton, $\mathrm{OH}$

\section{Education:}

- Bachelor of Science in Human Ecology

The Ohio State University, March 2009

- Dietetic Internship, University of Kentucky, July 2010

Professional Positions:

- Teaching Assistant, University of Kentucky, Department of Nutrition and Food Science 2009 - 2011

Scholastic Honors:

- Outstanding Dietetic Intern, 2010 